\title{
Quantitative Modelling of Climate
}

Change Impact on Hydro-climatic

\section{Extremes}

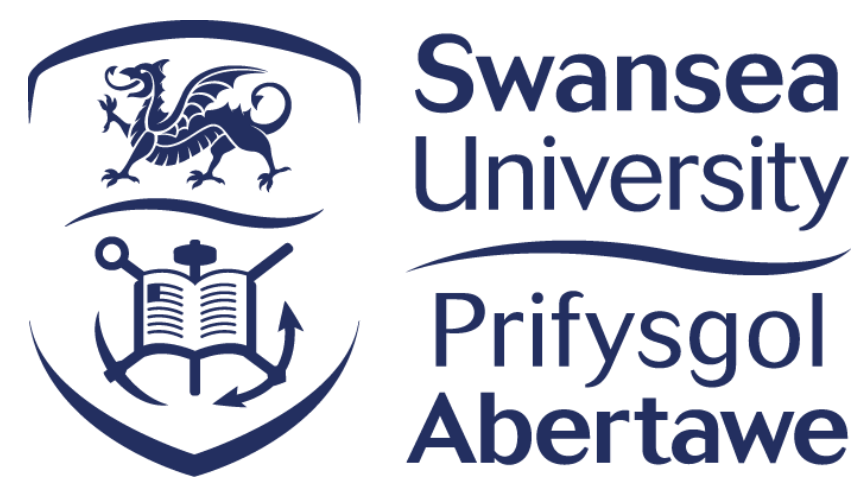

\section{Han Wang}

Faculty of Science and Engineering

Swansea University

Submitted to Swansea University in fulfilment of the requirements for the degree of Doctor of Philosophy 

I would like to dedicate this thesis to my beloved parents. 



\section{Declaration}

This work has not previously been accepted in substance for any degree and is not being concurrently submitted in candidature for any degree.

Signed:

Date: $03 / 11 / 2021$

This thesis is the result of my own investigations, except where otherwise stated. Other sources are acknowledged by footnotes giving explicit references. A bibliography is appended.

Signed:

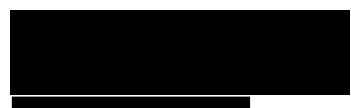

Date: $03 / 11 / 2021$

I hereby give consent for my thesis, if accepted, to be available for photocopying and for inter-library loan, and for the title and summary to be made available to outside organisations.

Signed:

Date: $03 / 11 / 2021$

The University's ethical procedures have been followed and, where appropriate, that ethical approval has been granted.

Signed:

Date: $03 / 11 / 2021$ 



\section{Acknowledgements}

In the past four years of my Ph.D. research, I received a great deal of support and encouragement from many people which made the thesis possible.

Firstly, I would like to express my sincerest gratitude to my supervisor, Assoc. Prof. Dr Yunqing Xuan, for his constant guidance and support, great encouragement and patience through each stage of my Ph.D. progress. At the initial phase of my research where I was puzzled by many unsure concerns, Dr Xuan guided me in a clear direction and supported me with his insightful knowledge. In the following years, I learned a lot from our regular meetings and discussions where not only many technical problems were solved promptly but also his intelligent and inspiring ideas motivated me to explore this challenging but exciting research area of extreme quantification under climate change. Meanwhile, Dr Xuan trained me by providing me with many opportunities for presenting my research work to both general public and research communities, communicating and collaborating with peer researchers, which helps me immensely build up my scientific research career. Apart from the research, Dr Xuan also cares about my wellbeing and self-development. I could not have current accomplishments without his continued mentoring and support.

I wish to thank Prof. Dr Harshinie Karunarathna for her always help and encouragement. Her expertise, comments and feedback were invaluable and important to my work. And I also wish to thank Assoc. Prof. Dr Leonardo Alfonso and Assoc. Prof. Dr Schalk Jan van Andel for their always support which made it possible for me to continue my study on decision making under uncertainty in the context of flood forecasting and early warning.

I would also like to thank all examination committee members, Assoc. Prof. Dr Gerald Corzo Perez and Dr Ji Li for providing me with an inspiring and enjoyable viva, and for your constructive comments that helped me to improve the quality of this thesis.

Thank you all my colleagues in Energy and Environment Research Group who always provide a friendly and enjoyable atmosphere for both working and recreation. It was a great pleasure to work with you all and I enjoyed our outdoor social gatherings.

I would like to thank the Centre of Hydrology and Ecology (CEH), The Bureau of Meteorology, Australia, European Centre for Medium-Range Weather Forecasts (ECMWF) and Met Office Hadley Centre for providing me research datasets, the Southern Regional Hydrometeorological Centre (SRHC), Vietnam for providing the data to support the case study of compound flood analysis in Ho Chi Minh City.

Thank you my housemate Yanan Sun for accompanying me especially during a hard time of the Covid-19 pandemic. The old happy time we studied, cooked, had fun, travelled, and much 
more...were so unforgettable and will remain as treasure in my memory. I would also like to thank my whole life friends, Ji Xiaochen, Li Tianxia and Shen Lin, for our great friendship since high school. Your kindness, enthusiasm and positive attitude to life encouraged me to keep improving myself in my way.

Last but not least, I wish to thank my dear parents for their unconditional love, understanding and support throughout my life which help me enormously to pursue my dream and interests and accomplish each milestone successfully. 


\begin{abstract}
In recent decades, climate change has caused a more volatile climate leading to more extreme events such as severe rainstorms, heatwaves and floods which are likely to become more frequent. Aiming to reveal climate change impact on the hydroclimatic extremes in a quantitative sense, this thesis presents a comprehensive analysis from three main strands.

The first strand focuses on developing a quantitative modelling framework to quantify the spatiotemporal variation of hydroclimatic extremes for the areas of concern. A spatial random sampling toolbox (SRS-GDA) is designed for randomizing the regions of interest (ROIs) with different geographic locations, sizes, shapes and orientations where the hydroclimatic extremes are parameterised by a nonstationary distribution model whose parameters are assumed to be time-varying. The parameters whose variation with respect to different spatial features of ROIs and climate change are finally quantified by various statistical models such as the generalised linear model. The framework is applied to quantify the spatiotemporal variation of rainfall extremes in Great Britain (GB) and Australia and is further used in a comparison study to quantify the bias between observed and climate projected extremes. Then the framework is extended to a multivariate framework to estimate the time-varying joint probability of more than one hydroclimatic variable in the perspective of non-stationarity. A case study for evaluating compound floods in Ho Chi Minh City, Vietnam is applied for demonstrating the application of the framework.
\end{abstract}

The second strand aims to recognise, classify and track the development of hydroclimatic extremes (e.g., severe rainstorms) by developing a stable computer algorithm (i.e., the SPER toolbox). The SPER toolbox can detect the boundary of the event area, extract the spatial and physical features of the event, which can be used not only for pattern recognition but also to support AI-based training for labelling/cataloguing the pattern from the large-sized, grid-based, multi-scaled environmental datasets. Three illustrative cases are provided; and as the front-end of AI study, an example for training a convolution neural network is given for classifying the rainfall extremes in the last century of GB.

The third strand turns to support decision making by building both theory-driven and datadriven decision-making models to simulate the decisions in the context of flood forecasting and early warning, using the data collected via laboratory-style experiments based on various information of probabilistic flood forecasts and consequences.

The research work demonstrated in this thesis has been able to bridge the knowledge gaps in the related field and it also provides a precritical insight in managing future risks arising from hydroclimatic extremes, which makes perfect sense given the urgent situation of climate change and the related challenges our societies are facing. 



\section{List of Publications}

\section{Items marked by superscript 来 are directly related to the subject addressed in this thesis. Items marked by superscript * indicate corresponding author of the publication.}

\section{Journal Papers:}

[1] 莱 Wang, H., \& Xuan, Y. (2020). SRS-GDA: A spatial random sampling toolbox for gridbased hydro-climatic data analysis in environmental change studies. Environmental Modelling \& Software, 124, 104598. https://doi.org/10.1016/j.envsoft.2019.104598.

[2] 来 Wang, H., \& Xuan, Y. (2020). Spatial variation of extreme rainfall observed from two century-long datasets. Geophysical Research Letters, 48, e2020GL091933. https://doi.org/10.1029/2020GL091933.

[3] 来 Wang, H., \& Xuan, Y. (2021). An area-orientated analysis of the temporal variation of extreme daily rainfall in Great Britain and Australia, Weather and Climate Extremes (under review).

[4] 来 Wang, H., \& Xuan, Y. (2021). Modelling the spatial variation of catchment-oriented extreme rainfall in England and Wales. Atmospheric Research (revised and resubmitted).

[5] 事 Wang, H., Tran, T. V. T., Xuan, Y, Couasnon, A., Scussolini, P., Linh, N. L., NguyenHong, Q., Reeve, D. Nonstationary Multivariate Framework: A Case Study of Compound Flooding Simulation in Ho-Chi-Minh City, Vietnam (in preparation).

[6] 来 Wang, H., \& Xuan, Y. Spatial Pattern Extraction and Recognition (SPER) toolbox for grid-based datasets and its application to machine learning technique (in preparation).

[7] Ma, L., Ni, J., Fleskens, L., Wang, H.*, \& Xuan, Y. (2021). Modelling fertilizer use in relation to farmers' household characteristics in Three Gorges Reservoir area, China. Agriculture, 11, 472. https://doi.org/10.3390/agriculture11060472.

[8] Couasnon, A., Scussolini, P., Tran, T. V. T., Eilander, D., Muis, S., Wang, H., Keesom, J., Dullaart, J., Xuan, Y., Nguyen Hong, Q., Winsemius, H. and Ward, P.J. (2021). Seasonal dependence between rainfall and sea levels modulates flood risk in Ho Chi Minh City. Water Resources Research (revised and resubmitted).

[9] 事 Wang, H.*, Alfonso, L., van Andel, S. J., Pappenberger, F., \& Xuan, Y. Analyzing decision-making under uncertainty in the context of flood forecasting and early warning using Decision Trees and Cumulative Prospect Theory (in preparation).

[10] Zhang, J., Hou, G., Wang, H., Zhao, Y., \& Huang, J. (2018). Operation feature extraction of flood discharge structure based on improved variational mode decomposition and variance 
dedication rate. Journal of Vibration and Control, 26(3-4), 229-240. https://doi.org/10.1177/1077546319878542.

\section{Book Chapters:}

[11] 来 Wang, H., \& Xuan, Y. (2021). Deep learning of extreme rainfall patterns using enhanced spatial random sampling with pattern recognition. Hydroinformatics. AGU.

\section{Conference Papers and Presentations:}

[12] Wang, H., \& Xuan, Y. (2022). Study of the Spatial Variation of Extreme Rainfall in Great Britain and Australia using the SRS-GDA Toolbox. The 11th International Conference on Weather Radar and Hydrology (WRaH 2022), Nanjing, China.

[13] 事 Xuan, Y., \& Wang, H. (2020). Quantitative modelling of hydroclimatic extremes. Oman's International Conference on Water Engineering and Management of Water Resources, Towards a Sustainable Water Future. Oman. ISBN: 978-0-7277-6525-3.

[14] 来 Wang, H., \& Xuan, Y. (2020). Temporal and spatial variation of extreme rainfall in Great Britain and Australia using the SRS-GDA toolbox. 6th IAHR Europe Congress, Warsaw, Poland, Feb 15 - 19, 2021. ISBN: 978-83-66847-01-9.

[15] 来 Wang, H., \& Xuan, Y. (2020). A Comprehensive Analysis of Nonstationarity and Spatial Variation of Rainfall Extremes in Great Britain and Australia using Century-long Datasets. AMS 101st Annual Meeting, United States, Jan 18 - 22, 2021.

[16] 坢 Wang, H., \& Xuan, Y. (2019). A multi-scale analysis of the correlation in representing flood inducing extreme precipitations from observation and climate model simulation. ${ }^{\text {th }}$ IMA International Conference on Flood Risk, Swansea, Wales, Sep 12 - 13, 2019.

[17] 来 Wang, H., Alfonso, L., van Andel S. J., \& Xuan, Y. (2018). Exploring Decision Making under Uncertainties in Context of Climate Change. RMetS Evolution of Science: Past, Present and Future Students \& Early Career Scientists Conference, Jul 4 - 6, 2018.

\section{Developed toolboxes:}

[1] SRS-GDA toolbox: Spatial Random Sampling for Grid-based Data Analysis (doi: 10.5281/zenodo.4044626). Available at the GitHub (https://github.com/wanghan924/SRSGDA_Toolbox.git).

[2] SPER toolbox: Spatial Pattern Extraction and Recognition. Available at the GitHub (https://github.com/wanghan924/SPER-toolbox). 


\section{Contents}

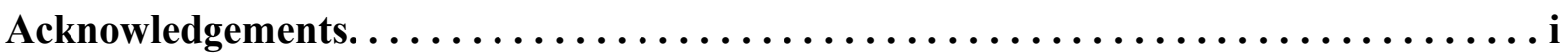

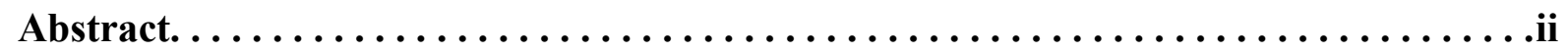

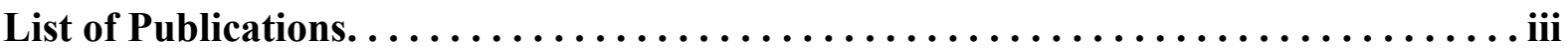

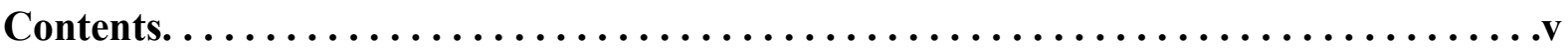

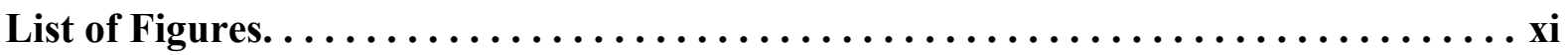

List of Tables. ....................................

Symbols and Abbreviations. $\ldots \ldots \ldots \ldots \ldots \ldots \ldots \ldots \ldots \ldots \ldots \ldots \ldots \ldots \ldots \ldots \ldots \ldots \ldots$

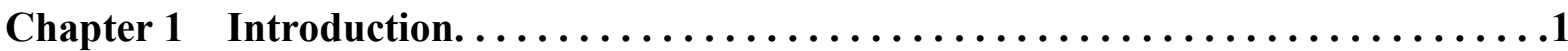

1.1 Background and motivation 1

1.2 Scope of research 2

1.2.1 Quantification of hydroclimatic extremes from observed records 3

1.2.2 Climate modes of variability and hydroclimatic extremes 10

1.2.3 Climate models, climate projections and hydroclimatic extremes 12

1.2.4 Computer vision and its potential application in recognising and classifying hydroclimatic extremes $\quad 15$

$\begin{array}{lll}1.3 & \text { Research objective and questions } & 18\end{array}$

$\begin{array}{lll}1.4 & \text { Study datasets } & 20\end{array}$

1.5 Layout of thesis 22

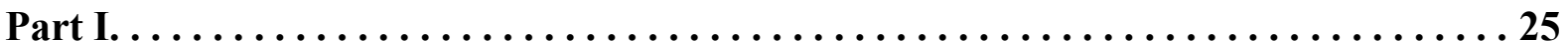

Chapter 2 The Development of a Spatial Random Sampling Toolbox. . . . . . . . . 29 
2.1 Introduction $\quad 29$

2.2 The design of SRS-GDA toolbox 30

2.2.1 Generating the grid-based overall boundary (OB) map 34

2.2.2 Sampling setup 35

2.2.3 Sampling processing and validation 36

2.3 An example application of the toolbox 39

2.3.1 Example dataset 39

2.3.2 Application of the SRS-GDA toolbox 40

$2.4 \quad$ Summary and remarks $\quad 46$

Chapter 3 Modelling Spatial Variation of Rainfall Extremes Using an ROI-based

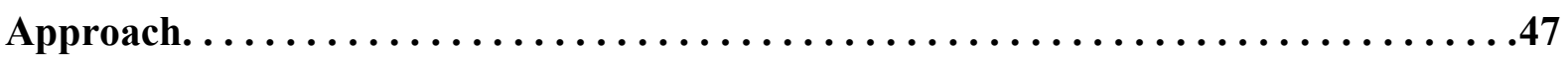

$\begin{array}{lll}3.1 & \text { Introduction } & 48\end{array}$

3.2 Methodology 49

3.2.1 ROI generation, catchment identification and AMDR extraction 50

3.2.2 Fitting the extracted AMDRs using GEV distribution 54

3.2.3 Goodness of fit tests 55

3.2.4 Analysing the spatial distribution of the location-scale parameters 58

3.3 Results of goodness of fit tests 60

3.4 Qualitative analysis of the spatial variation of ROI-based study 64

3.4.1 GEV parameter variation over geographical locations 64

3.4.2 Variation of GEV parameters with regards to the area size 66

3.4.3 Variation of GEV parameters due to change of ROI shape 67

3.4.4 Quantitative modelling of the spatial variation 69

3.5 Qualitative analysis on the spatial variation of catchment-based study 81

3.5.1 Spatial features of catchments and simulation results of GEV model 81

3.5.2 Spatial variation of AMDR with respect to location and elevation 84

3.5.3 Spatial variation of AMDR with respect to catchment size 86

3.5.4 Spatial variation of AMDR with respect to catchment orientation and shape 89

3.6 The link between the spatial variations of GEV parameters and the large-scale climatology of rainfall variation $\quad 92$

$\begin{array}{lll}3.7 & \text { Summary and remarks } & 97\end{array}$ 
$\begin{array}{lll}4.1 & \text { Introduction } & 102\end{array}$

$\begin{array}{lll}4.2 & \text { Methodology } & 104\end{array}$

4.2.1 Block bootstrapping Mann-Kendall (BBS-MK) test 104

4.2.2 Stationary generalised extreme value model (S-GEV) 106

4.2.3 Nonstationary generalized extreme value model (NS-GEV) 107

4.2.4 Goodness of fit and performance of the S-GEV and NS-GEV models 110

4.3 Results 111

4.3.1 Results of BBS-MK test 111

4.3.2 Selection of stationary and nonstationary models and spatial nonstationary patterns 113

4.3.3 Spatial variation of nonstationary patterns over ROI size 116

4.3.4 Spatial variation of nonstationary patterns over ROI shape 118

$\begin{array}{lll}4.4 & \text { Implication on return period } & 120\end{array}$

$\begin{array}{lll}4.5 & \text { Summary and remarks } & 122\end{array}$

Chapter 5 Spatiotemporal Variation of Climate Projection Extremes Compared

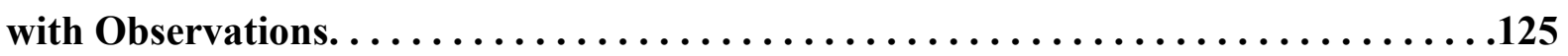

$\begin{array}{llr}5.1 & \text { Introduction } & 126\end{array}$

$\begin{array}{lll}5.2 & \text { Methodology } & 127\end{array}$

$\begin{array}{llr}5.3 & \text { Results } & 129\end{array}$

5.3.1 Link to ERA20CM projected extreme rainfall in GB 129

5.3.2 Link to UKCP18 projected extreme rainfall in GB 135

$\begin{array}{lll}5.4 & \text { Summary and remarks } & 140\end{array}$

Chapter 6 Nonstationary Multivariate Framework: A Case Study of Compound Flooding Simulation in Ho-Chi-Minh City, Vietnam. . . . . . . . . . . . 143

6.1 Introduction 143

6.2 A nonstationary framework of multivariate probability distribution analysis 146

$\begin{array}{lll}\text { 6.2.1 Trend and correlation analysis } & 147\end{array}$

6.2.2 Model marginal distributions of the series of data 148

6.2.3 Build copulas and calculate the joint probability 151 
6.2.4 Generate the quantiles 153

6.3 Case study 155

6.3.1 Study domain and data processing 155

6.3.2 Hydrodynamic modelling and simulation 157

$\begin{array}{lll}6.4 & \text { Results and discussion } & 159\end{array}$

6.4.1 Trend and correlation analysis 159

6.4.2 Stationary and nonstationary joint probability distribution 163

6.4.3 Scenarios generation and compound flood simulation in HCMC 167

$\begin{array}{lll}6.5 & \text { Summary and remarks } & 172\end{array}$

Part II. ........................................... 175

Chapter 7 Identification and Classification of Hydroclimatic Extremes Using Pattern Recognition and Convolution Neural Networks. . . . . . . . . . . 177

$\begin{array}{lll}7.1 & \text { Introduction } & 177\end{array}$

$\begin{array}{ll}7.2 & \text { The design of the SPER toolbox } \\ & 180\end{array}$

7.2.1 Thresholding segmentation and boundary detection 181

7.2.2 The algorithm for extracting and quantifying the spatial features of ROI 182

7.2.3 The algorithm for quantifying the hydroclimatic features of ROI 185

7.3 Illustrative Case Studies 186

7.3.1 Catchment-based analysis of extreme rainfall in England and Wales 186

7.3.2 Pattern recognition of daily rainfall over the last century in Great Britain 187

7.3.3 Tracing rainfall area and spatial distribution in 24 hours in Guangzhou, China

7.4 The front-end application for training a convolution neural network 196

$\begin{array}{lll}7.5 & \text { Summary and remarks } & 198\end{array}$

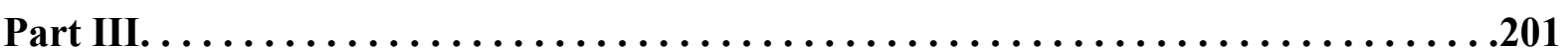

Chapter 8 Decision-making under Uncertainty in the Context of Flood Forecasting and

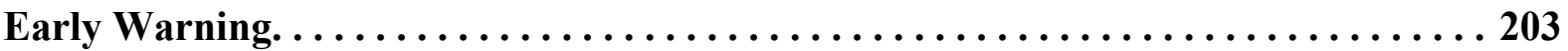

$\begin{array}{lll}8.1 & \text { Introduction } & 204\end{array}$

8.1.1 Experiment design and implement 207 
8.1.2 Two decision models 209

8.2 Reconstruction and improvement of two decision models 212

8.2.1 Improving the Cumulative Prospect Theory (CPT) model 212

8.2.2 Reconstructing the Decision Tree (DT) model 216

8.2.3 Evaluation of model performance 216

$\begin{array}{lll}8.3 & \text { Results } & 217\end{array}$

8.3.1 The new CPT model generation with data collected in Ex1 217

8.3.2 The new DT model generation with data collected in Ex1 220

8.3.3 Performance of the new CPT and DT models tested by data collected in Ex2 221

8.4 Application of new CPT and DT models 223

$\begin{array}{lll}8.5 & \text { Summary and remarks } & 225\end{array}$

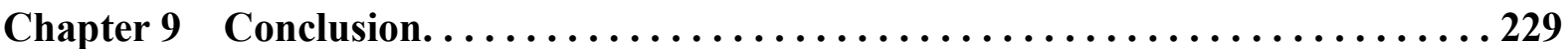

9.1 Innovations and contributions 229

$\begin{array}{lll}9.2 & \text { Future work } & 233\end{array}$

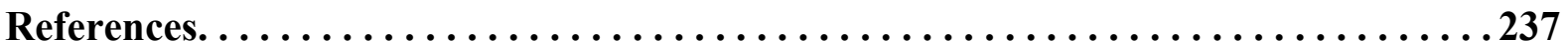

Appendix Data Processing and Toolbox Access................... 261 


\section{List of Figures}

Figure 1.1 Layout of the thesis.

Figure I.1 Quantitative modelling framework for analysing the spatiotemporal variation of hydroclimatic extremes.

Figure 2.1 The three basic steps and the corresponding inputs and outputs of the SRS-GDA toolbox.

Figure 2.2 The 'buffering' operation used to vary the ROI into different sizes (shown here in different border colours).

Figure 2.3 The process of shape-unconstrained random sampling method with ill-sample detection and removal. .38

Figure 2.4 Flowchart of shape-constrained sampling method. .38

Figure 2.5 General map of Great Britain with three resolutions: (a) $1 \mathrm{~km} \times 1 \mathrm{~km}$ (b) $5 \mathrm{~km} \times 5 \mathrm{~km}$ (c) $10 \mathrm{~km} \times 10 \mathrm{~km}$. The difference in details and resolutions can be appreciated in the

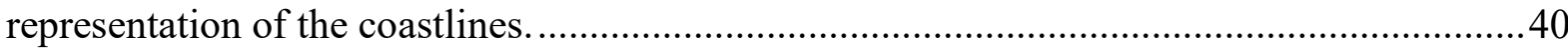

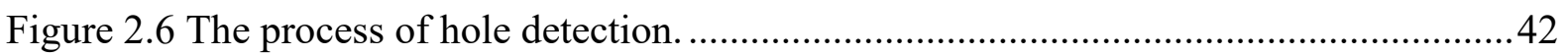

Figure 2.7 The process of generating samples by shape-constrained sampling method........ 43

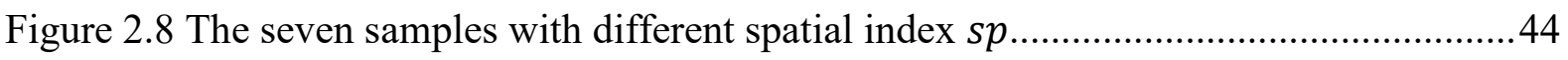

Figure 2.9 The dependencies on the locations, the shape index and the size of rainfall distribution over GB: (a) the east-west pattern and (b) the symmetric pattern with regards to the sampled shape and (c) the trend pattern with regards to the sampled area size as detected by using the toolbox discussed in this chapter. EP is short for "Exceedance Probability". ....46

Figure 3.1 Location of the catchments over England and Wales (a) and one catchment with its

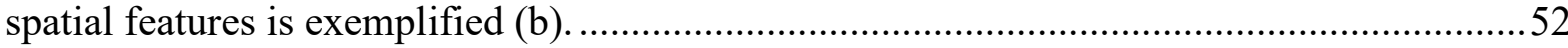

Figure 3.2 Zonal and meridional average parameter analysis in Great Britain (GB) and Australia (AU).

Figure 3.35000 iterations of KS test statistics in 88 ROIs of GB cases, where each title shows the $p$-value and the location index of ROI in bracket.

Figure 3.4 Histograms of the $p$-value of all ROIs (a) in GB and AU and all catchments (b) in England and Wales by KS and AD tests. 
Figure 3.5 L-moment ratio diagram of L-kurtosis versus L-skewness in GB (a) and AU (b). 63 Figure 3.6 Histograms and spatial variations of the three GEV parameters in GB (a) and AU (b).

Figure 3.7 The changes of meridional average location-scale parameters with the ROIs' size in GB (a) and AU (b) shown by colour scale.

Figure 3.8 The changes of meridional average location-scale parameters with the ROIs' shape in GB (a) and AU (b) shown in the colour scale.

Figure 3.9 Histograms of GEV parameters (meridional averaged $\mu$ and $\sigma$ ) of GB and AU cases.

Figure 3.10 The selection process for the optimal structure of GLM for meridionally averaged $\mu$ and $\sigma$ in GB and AU.

Figure 3.11 Visualisation of the GLMs fitted to the meridional average GEV $\mu$ and $\sigma$ parameters as a colour-scale plot for GB (a) and a contour plot for AU (b) whose contours are picked up at the same stops of the values and their changes with respect to the geographic location and size. .76

Figure 3.12 A scatter plot (a) and a normal quantile plot (b) for revealing the difference between the actual GEV parameters and the modelled GEV parameters. .78

Figure 3.13 Nash-Sutcliffe efficiency (NSE) coefficient of 10-fold cross-validation (CV) method for evaluating the performance of four GLMs.

Figure 3.14 The variograms for evaluating spatial continuity of GEV parameters $\mu$ and $\sigma$ estimated by GEV and GLM models of GB (a) and AU (b) cases fitted by Spherical model.81

Figure 3.15 Spatial features of study catchments.

Figure 3.16 Spatial variation of AMDR with respect to catchment location represented by GEV parameters $\xi(\mathrm{a}), \sigma$ (b) and $\mu$ (c); and the relation between these three parameters and the catchment elevation $(H)$ is presented in d,e,f respectively. 86

Figure 3.17 Categories of five groups according to the level of AMDR and GEV parameters $\sigma$ and $\mu$ changing with catchment size in the catchments with "Moderate", "High" and "Extreme" levels of AMDR. 88

Figure 3.18 GEV parameters $\sigma$ and $\mu$ change over orientation $\omega$ (a) and shape $s p$ (b) of catchments in the three groups where "LLR" is short for "local linear regression" and the directions "North", "West" and "East" are abbreviated as "N", "W" and "E". 92

Figure 3.19 The variograms for comparing spatial continuity of GEV parameters $\mu$ and $\sigma$ with spatial continuity of areal annual rainfall (AAR) 's mean and their standard deviation (SD) of GB (a) and AU (b) cases fitted by Spherical model.

Figure 3.20 Comparison between the climatic variables (the average annual rainfall and its standard deviation) and GEV parameters $\mu$ and $\sigma$ in GB (a) and AU (b) cases where the colour denotes the value of averaged annual rainfall or the GEV parameter $\mu$, and the size of the circles denotes the value of the standard deviation of the annual rainfall or the GEV parameter $\sigma \ldots 97$ 
Figure 4.1 Trends of AMDR detected in GB (a) and AU (b), represented by Kendall's tau.112 Figure $4.2 p$-values of all ROIs for temporary variation analysis in GB and AU.

Figure 4.3 Spatial distribution of ROIs with the size of $500 \mathrm{~km}^{2}$ and relatively rounded shape in terms of (1)the best-selected model type in GB (a) and AU (e); (2) the best fitted GEV type in GB (b) and AU (f); (3) the changes of location (c and g) and scale parameters ( $d$ and $h$ ) in percentage within the record periods (113 years for GB and 129 years for AU) and please noted that white colour ("w" shown in the colour bar) indicates the ROI with no change of the parameters. 115

Figure 4.4 Spatial distribution of ROI groups whose parameters $(\mu 0, \mu 1, \sigma 0, \sigma 1)$ change with the increase of ROI size in GB (abcd) and AU (efgh). Please note that the white colour indicates the ROI with the insignificant change of the parameters. Noted that "w" shown in the colour bar indicates the colour white which means the changes of the parameter is zero. ...118

Figure 4.5 Both baseline and time-varying parameters $(\mu 0, \mu 1, \sigma 0, \sigma 1)$ change over the ROI shape indicated by the index of $s p$ in GB (ab) and AU (cd). The horizontal axis indicates the location index of the ROIs and the colour bar shows the values of parameters. ...................120

Figure 4.6 Nonstationary return periods corresponding to the return levels estimated by the stationary model and the spatial distribution referring to 1 -in-5 years, 1 -in-25 years and 1-in50 years in GB (a) and AU (b). An overall comparison between the nonstationary and stationary return levels corresponding to the same return year is presented as a boxplot in $\mathrm{c}$ where the upper panel shows the GB case and the lower shows the AU case. Noted that "w" in the colour bar indicates the colour white which means there is no difference between stationary and nonstationary return periods.

Figure 5.1 The location of three study areas (i.e., three grids of $0.4^{\circ} \times 3.15^{\circ}$ ). The green area corresponding to the left vertical axis shows the cover of GB and grids in the GEAR dataset while the yellow marks * corresponding to the right vertical axis indicate the edge of grids in the ERA20CM dataset. 126

Figure 5.2 Methodology of the study. 129

Figure 5.3 Stationary (S) vs. nonstationary (NS) return levels for three time slices (i.e., the year 1910, 1960 and 2010) estimated by different methods by using both the observed (GERA) and projected (ERA20CM) AMDR in the study area of England. 130

Figure 5.4 Stationary (S) vs. nonstationary (NS) return levels for three time slices (i.e., the year 1910, 1960 and 2010) estimated by different methods by using both the observed (GEAR) and projected (ERA20CM) AMDR in the study area of Scotland. 133

Figure 5.5 Stationary (S) vs. nonstationary (NS) return levels for three time slices (i.e., the year 1910, 1960 and 2010) estimated by different methods by using both the observed (GEAR) and projected (ERA20CM) AMDR in the study area of Wales. 134

Figure 5.6 Comparison between stationary GEV of observed and climate projected AMDR in the study area of England (a); and comparison of stationary (S) and nonstationary (NS) return levels of observed (GEAR) and climate projected (UKCP18) AMDR at the specific years 1990 and 2000 (b). 137 
Figure 5.7 Comparison between stationary GEV of observed and climate projected AMDR in the study area of Wales (a); and comparison of stationary (S) and nonstationary (NS) return levels of observed (GEAR) and climate projected (UKCP18) AMDR at the specific years 1990 and 2000 (b).

Figure 6.1 Nonstationary framework of multivariate probability distribution analysis. MMR is the monthly maximum rainfall; MMS is the monthly maximum skew surge. 147

Figure 6.2 Joint density level of quantile curves corresponding to the probability from 0.01 to 0.99

Figure 6.3 Study area (Ho Chi Minh City) where six rain gauges for collecting the daily rainfall over the city centre are denoted by red circles and a water level gauge by a yellow rectangle.

Figure 6.4 Trend analysis of correlation structure between MMS and MMR in March (left) and April (right) where the correlation coefficients $\tau$ of Kendall test and $\rho$ of Spearman test are depicted by blue curves and p-values are indicated by red bars and the significance level (0.05) is shown in dashed black line.

Figure $6.5 p$-value of two rolling window correlation tests (Kendall test and Spearman test) between monthly maxima of daily rainfall and skew surge in March and April.

Figure 6.6 Mean sea level pressure and wind at $850 \mathrm{mb}$ for March (a) and April (b) averaged from 1979 to 2020 using the ERA5 dataset.

Figure 6.7 Distribution of monthly maximum time series of skew surge (a) and rainfall (b) (1980-2017). On each box, the central mark indicates the median, and the bottom and top edges of the box indicate the $25^{\text {th }}$ and $75^{\text {th }}$ percentiles, respectively, and the outliers are indicated by '+'. 164

Figure 6.8 Quantiles corresponding to different joint cumulative probability $(\mathrm{p}=0.1,0.5$ and 0.9 ) of the monthly maximum skew surge and rainfall under different assumptions in the selected four months where the best-fitted distributions of two marginals (MMS and MMR) are shown in the left and lower panels of each figure respectively: if the best-fitted distribution is stationary, the comparison between empirical and best-fitted distribution are shown while if it is nonstationary, only the best-fitted distribution is plotted and colour of curves indicates the

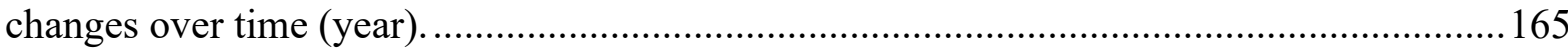

Figure 6.9 Two typical astronomical tide profiles in March. ................................................ 169

Figure 6.10 Three typical astronomical tide profiles in October....................................... 170

Figure 6.11 Flood inundation maps compared between the stationary and nonstationary cases

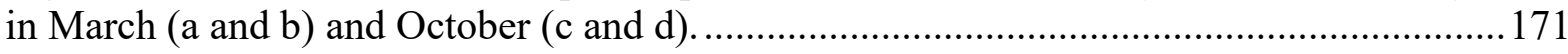

Figure 7.1 SPER: Spatial Pattern Extraction and Recognition Toolbox. 181

Figure 7.2 Two example sketches of ROI where $f(x, y) \geq 35$ without (a) and with (b) hole. 182

Figure 7.3 An example of ROI. 184 
Figure 7.4 An example of ROI with its core

Figure 7.5 The histograms of the number of daily rainfall patterns in England, Wales and Scotland displayed by different colours with respect to $s p$ and $\omega$ at three sizes $(\mathrm{C} 1, \mathrm{C} 2$ and C3)

Figure 7.6 The changes of the scattered area of rainfall centres in 24 hours 194

Figure 7.7 Characteristics of the largest rainfall area (a) and its moving track (b) in 24 hours.

Figure 7.8 Four labels corresponding to the daily rainfall patterns: no rainfall (L0), concentric pattern (L1) where its sp is near 1.0, elongated pattern (L2) and compound pattern (L3) which has both concentric and elongated types (a); and the training and validation loss (blue and black lines) and validation accuracy (orange lines) of CNN (b). 197

Figure 8.1 Procedure to build and apply the new CPT model based on Cumulative Prospect Theory (CPT). 213

Figure 8.2 Flood forecast probability versus the modelled and observed weighted probabilities for prospects $\mathrm{P}_{\mathrm{y}}$ and $\mathrm{P}_{\mathrm{n}}$. 219

Figure 8.3 Optimal decision tree, where Probability refers to the probability of flood occurrence $\mathrm{p}$, and $\mathrm{r}$ refers to the cost/loss ratio.

Figure 8.4 The number of majority decisions observed and simulated by the new CPT and DT models.

Figure 9.1 Accumulative rainfall (mm) in Zhengzhou from 18 to 27 July 2021 and this figure is produced by ECHO/European Commission (source: https://erccportal.jrc.ec.europa.eu/ECHO-Products/Maps\#/maps/3780 Accessed: 2021-11-02) 



\section{List of Tables}

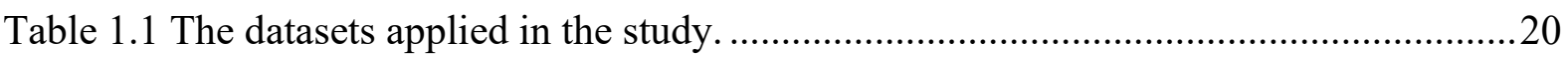

Table 2.1 Five example samples generated by shape-unconstrained sampling method..........41

Table 2.2 Five example samples generated by shape-constrained sampling method..............42

Table 2.3 Comparison of the indicative speed of the two sampling methods: Method 1 the shape-unconstrained method and Method 2 the shape-constrained method. Note that the numbers are obtained on the test PC and for comparing the relative speed difference...........43

Table 3.1 ROIs for analysing the spatial variations in GB and AU.....................................50

Table 3.2 All attempts of proposed GLM form $\boldsymbol{X}$ for two parameters of two cases. .............. 70

Table 3.3 Coefficients of four GLMs and their $p$-values (shown in brackets)......................73

Table 4.1 Stationary and nonstationary GEV models and the estimation methods.............. 107

Table 5.1 Estimated parameters of stationary (S) and nonstationary (NS) GEV models of both observed and projected AMDR in the study area of England. .......................................... 131

Table 5.2 Estimated parameters of stationary (S) and nonstationary (NS) GEV models of both

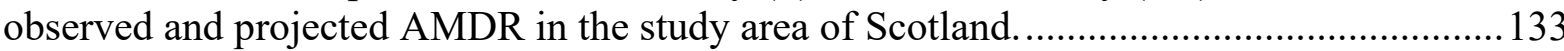

Table 5.3 Estimated parameters of stationary (S) and nonstationary (NS) GEV models of both observed and projected AMDR in the study area of Wales........................................... 135

Table 5.4 Estimated parameters of stationary (S) and nonstationary (NS) GEV models of both

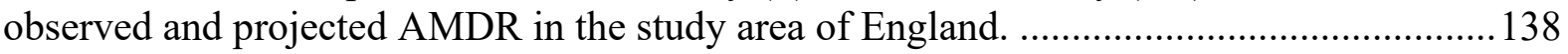

Table 5.5 Estimated parameters of stationary (S) and nonstationary (NS) GEV models of both observed and projected AMDR in the study area of Wales................................................ 140

Table 6.1 Stationary (S) and nonstationary (NS) candidate distributions for time series. For the

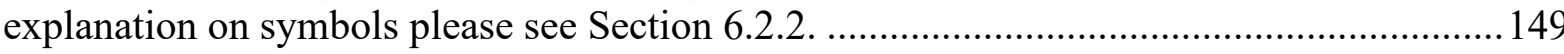

Table 6.2 Test and estimation results of monthly maximum rainfall (MMR) and skew surge

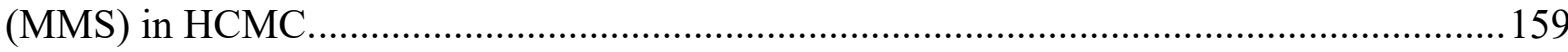

Table 6.3 The scenarios for compound flood simulation. .............................................. 168

Table 7.1 Specific dominating patterns of daily rainfall in England, Wales, and Scotland catalogued at three sizes. 
Table 7.2 Temporal change of specific rainfall patterns in three countries in three size

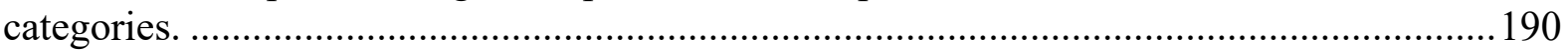

Table 8.1 Sample of instances for DT model building. .....................................................216

Table 8.2 Expected values of each prospect compared with their observed certainty equivalents.

Table 8.3 Parameters of new CPT models for losses (where KT92 refers to the original parameters reported in Tversky and Kahneman (1992)) .................................................220

Table 8.4 Decisions taken by participants and simulated results by three models. ...............224 


\title{
Symbols and Abbreviations
}

\author{
Spatial Random Sampling for Grid-based Dataset Analysis (SRS-GDA) toolbox \\ $L \quad$ Geometric centre $(x, y)$ of the sample \\ $S \quad$ Size of the sample \\ sp Shape index of the sample
}

Generalised Extreme Value (GEV) model

$\begin{array}{ll}\mu & \text { Location parameter } \\ \sigma & \text { Scale parameter } \\ \xi & \text { Shape parameter } \\ \mu_{0}, \mu_{1} & \text { Nonstationary location parameter } \\ \sigma_{0}, \sigma_{1} & \text { Nonstationary scale parameter }\end{array}$

Generalised Linear Model (GLM)

$\beta$

Coefficient of predictors

$X$

The combined structure of predictors

Spatial Pattern Extraction and Recognition (SPER) toolbox

$\omega \quad$ Orientation of the sample

Decision-making in the context of flood forecasting and early warning

$r$

Cost/loss ratio

$p$

Flood probability of forecast

$P_{y}, P_{n}$

Prospects of decisions

$D_{y}, D_{n}$

Decisions

\section{Abbreviations}

ARF

AAR

AAs

AD
Areal reduction factor

Area annual rainfall

Anthropogenic aerosols

Anderson-darling

xix 
ADAM

AIC

AMDR

ANN

AU

BBS-MK

BIC

B-MCMC

CE

$\mathrm{CEH}$

$\mathrm{CM}$

CMIP5

CMIP6

$\mathrm{CNN}$

CP

CPT

CV

DDS

DT

ECMWF

ENSO

EP

FFEW

FRM

GB

GCM

GDAL

GEAR

GEV

GHGs

GLM
Australian Data Archive for Meteorology

Akaike information criterion

Annual maximum daily rainfall

Artificial neural network

Australia

Block bootstrapping Mann-Kendall

Bayesian information criterion

Bayesian Markov-Chain Monte Carlo

Certainty equivalent

Centre for Ecology \& Hydrology

Climate model

Coupled model intercomparison project phrase 5

Coupled model intercomparison project phrase 6

Convolutional neural network

Climate projections

Cumulative prospect theory

Cross-validation

Dynamical downscaling

Decision trees

European Centre for Medium-Range Weather Forecasts

El Niño-southern oscillation

Exceedance probability

Flood forecasting early warning

Flood risk management

Great Britain

General circulation models

Geospatial Data Abstraction Library

Gridded Estimates of daily Areal Rainfall

Generalized extreme value

Greenhouse gases

Generalised linear model 
GOF

GPM

GRIB

HDF

HEPEX

HPC

IOD

KED

KS

LAM

LL

LM

LU

MFB

ML

NAO

NETCDF

NGR

NSE

NS-GEV

NS-RP

OB

POT

$\mathrm{RCM}$

ROI

SD

S-GEV

SPEI

S-RP

SRS-GDA

SST
Goodness of fit

Global precipitation measurements

Gridded Binary

Hierarchical data format

Hydrological ensemble prediction experiments

High-performance computation

Indian Ocean dipole

Kriging with external drift

Kolmogorov-Smirnov

Local area model

Log-likelihood

L-moments

Land use

Mean-field bias

Maximum likelihood

North Atlantic oscillation

Network common data format

National grid reference

Nash-Sutcliffe efficiency

The nonstationary generalised extreme value model

Nonstationary return period

Overall boundary

Peak over threshold

Regional climate model

Region of interest

Standard deviation

The stationary generalised extreme value model

Precipitation Evaporation Index

Stationary return period

Spatial Random Sampling for Grid-based Data Analysis

Sea surface temperature 


\section{Chapter 1 Introduction}

\subsection{Background and motivation}

It is well established that climate change causes a more volatile climate and hence more extreme storms and flooding, more frequent the occurrence of these events or the combination of both (Hall et al., 2014; Hirabayashi et al., 2013; Milly et al., 2002). However, it is yet to know such impact in a quantitative sense, which has caused great difficulties in both designing flood defence structures as well as in evaluating their reliability and effectiveness. A most recent, yet classical example was the aftermath of the 2015 Christmas severe flooding in North England (Spencer et al., 2018) where such questions were raised but failed to be answered for another time. Many so-called "climate-proof" design measures hallmarked by incorporating an arbitrary "safe" margin can neither be scientifically validated nor be economically justified. Therefore, there is an urgent need to study how the changing hydroclimatic extremes affect the engineering standard design procedure in a quantitative way.

Conventionally, the design of water engineering structures relies on the understanding of the dynamics and behaviour of these extremes such as extreme storms, floods, by applying a frequency analysis with long term historical records. For example, flood defence structures are designed to withhold floods up to a certain threshold, e.g. flood size, which in turn is determined by fitting historical extremes such as annual maximum flood peaks using a probability distribution whose parameters are assumed to be stationary (Coles and Tawn, 1996; Mannshardt-Shamseldin et al., 2010; Miniussi et al., 2020; Morrison and Smith, 2002; Szulczewski and Jakubowski, 2018). As it has become increasingly clear that climate change has already altered the environment hence the flooding process, such a stationary view needs to be changed (Herring et al., 2018). 
Further, although the impact of climate change has been appreciated globally, it is far from being homogeneous. For example, whilst certain regions may suffer more severe flooding, others simply observed more water deficiencies (Güneralp et al., 2015; Lehner et al., 2006). Although many hydrology phenomena such as the precipitation process are part of the global hydrological cycle and hence (laterally) boundless, the local and regional changes which directly affect human society are of great concern of stakeholders and policymakers (Jha et al., 2012). Therefore, understanding the spatial variability of the changing hydroclimatic extremes is another important dimension to be explored. In the past decades, research in the spatial variability in hydrology is usually based on gauge or station records to explore the correlations among different locations (Celleri et al., 2007; Ciach and Krajewski, 2006), which often is not comprehensive due to the scarce data and the lack of measurements. More recently, with the rapid advances in environmental monitoring technology, spatially disaggregated, grid-based hydro-climatic datasets have become gradually available with steady improvements in both accuracy and resolutions and their application has become a foundation to support further analyses on the environment or climate change both spatially and temporally (Nashwan et al., 2019; Peleg et al., 2018). Meanwhile, many environmental or hydroclimatic models nowadays are also tuned to make use of these new, grid-based, high-resolution datasets (Muthusamy et al., 2017b). However, it raises some important and new challenges as well. For example, these data usually come with spatial patterns and characteristics linked to certain changing factors that need to be diagnosed; those from climate projection models or field observations are often highlighted by the intermittent spatial variations, with often chaotic, nonstationary, multi-scale temporal distributions, which leads to difficulties in pattern identification using techniques such as deep machine learning and heavy overhead of computer programming.

\subsection{Scope of research}

This section presents an overview of the research in hydroclimatic extremes and recent progress in the last few decades. Hydroclimatology is defined as an interdisciplinary scientific field that synthesizes hydrology and climate, including the impacts that the movement, storage, and 
phase change of water between and within the terrestrial and atmospheric systems have on the global climate, and the impacts of climate patterns and changes on the global hydrological cycle (Wendland, 1987). The hydrological cycle involves the total process where liquid water moves from the land and water surface (e.g., ocean, river) to the atmosphere by evaporation and back in form of precipitation or snow (Kuchment, 2004). It is significantly linked with climate and can be affected by natural climate variability, e.g., the changes in the atmospheric temperature and radiation balance (Inglezakis et al., 2016). Warming of the climate system in recent decades has caused more frequent occurrences of extreme phenomena such as drought, flooding and extreme precipitation. It is because that the hydrological cycle may be strengthened by rising temperature with more evaporation and an increased ability of the atmosphere to hold water which leads to more intense precipitation events in some areas, but the extra areas of the world may suffer from significant reductions in precipitation, so-called droughts. Although it is unequivocal that many hydroclimatic extremes result from natural climate variability, the link between climate change (such as global warming) and local changes in precipitation especially at the extreme level has proved harder to establish (Dadson et al., 2019). Therefore, it is worth having a broader review of the recent progress in assessing climate change impacts on hydroclimatic extremes. Apparently, there can be many ways of carrying out such review, in this thesis, however, I shall focus on the following four strands:

1) identification and quantification of trends, cycles or characteristics in observed records of meteorological and hydrological extremes (section 1.2.1),

2) attribution analysis of historical climate change and variability which may lead to the changes of extreme phenomena (section 1.2.2),

3) evaluation of the climate projected effects on extremes (section 1.2.3) and

4) computer vision and its application in recognising hydroclimatic extremes (section 1.2.4).

\subsubsection{Quantification of hydroclimatic extremes from observed records}

Hydroclimatic extreme events are usually regarded as the most unexpected, unusual, rare but severe events such as heavy rainfall, floods, droughts, cyclones, avalanches, heat waves, and cold waves which can have a significant impact on socioeconomic aspects such as agriculture, 
water resources, ecosystem services and urban infrastructure, sometimes can cost the severe loss of human lives and properties. The term "extreme" is generally defined as the occurrence of a value of a weather or climate variable (e.g., heavy rainfall) above (or below) a threshold value near the upper (or lower) tail of the range of observed values of this variable (Seneviratne et al., 2012). Different disciplines have different selections of the threshold. For example, the Intergovernmental Panel on Climate Change (IPCC), defines the extremes as those are in the top (or below) $10 \%$ of severity for the given event type at a particular place and time of year. NCDC (NOAA's National Climatic Data Centre) defines extreme events (weather or climate) as those lying in the outermost $10 \%$ of the natural events based on the historical weather records (e.g., long-term rainfall records).

Despite the differences in defining extreme events, two approaches have been adopted in many studies and are considered as the most frequently used. The first approach, namely the "Annual Maxima (Minima) method", is to select the maximum or minimum value within a fixed period, e.g., a year from the observed data series (Jenkinson, 1955). As it only includes the single extreme event for every year, the second or third highest/lowest extreme events which may as well be very important are ignored, thereby some information will be lost especially when the data length is not long enough (e.g., $<50$ years). However, this approach is beneficial for water resources management which requires providing a single extreme event per year. To obtain a sufficient number of events and maintain as many characteristics of extreme events as possible, the other approach, namely the "Peak over Threshold (POT) method", was proposed to select extreme events based on a defined threshold (e.g., $>95^{\text {th }}$ or $99^{\text {th }}$ percentiles) during a period (Leadbetter, 1991). More extreme events will be involved by applying the POT method, but a further question needs to be addressed, e.g., how to choose an appropriate threshold, as an inappropriate selection will result in inaccurate quantification of hydroclimatic extremes; and how to prove that the data over the threshold are independent when applying a distribution type to fit them. Therefore, the selection of two approaches should be considered and normally based on the study objective and data availability.

\section{- The production of hydroclimatic data and the challenge for data application}


As hydroclimatic regimes vary in both spatial and temporal dimensions, spatiotemporal analysis is an essential approach to quantifying the characteristics of hydroclimatic extremes such as intensity, magnitude, duration, spatial extent and variability (Ljungqvist et al., 2016). A great effort has been made to provide the measurement of hydroclimatic variables such as rainfall with high spatial and temporal resolution due to the rapid developments in environmental monitoring technology. Historically, hydrometeorological datasets were often relatively scarce in space, and only available at a limited number of locations, because data collection was often restricted by technical conditions, instruments and means of storage. To make full use of these finite data, statistic methods were applied. In terms of rainfall data as an example, there are many statistical methods developed to translate point rainfall records usually collected from certain hydrological gauges or stations to basin/areal rainfall. For instance, the areal reduction factor (ARF) has been widely used, possibly under different names in different countries (NERC, 1977; US Weather Bureau, 1958). More recently, however, spatially disaggregated, grid-based hydroclimatic datasets have become gradually available with steady improvements in both accuracy and spatiotemporal resolution. A typical case is the NIMROD weather radar system deployed by the UK Met Office which can provide up to 1 $\mathrm{km} / 5$ min precipitation distribution over the country (Fairman Jr et al., 2017; Golding, 1998). Similarly, satellite-borne observations, such as the Global Precipitation Measurements (GPM; Islam et al., 2014; Ning et al., 2016) can now provide large scale coverage of the precipitation coverage in near real-time.

Another important source of producing high-resolution data is model-simulated hydroclimatic fields. In this case, rainfall, temperature as well as soil moisture fields generated by numerical weather models or climate models can be used to drive other model simulations. Practices of using the so-called coupled model approach started to gain momentum in the early 2000s when the numerical weather models and climate models were able to produce simulations with high enough spatial resolution, e.g., at tens of kilometres. As such, there have been plenty of studies since then, such as Bauer et al. (2015), Moufouma-Okia and Jones (2015) and many more inspired by the Hydrological Ensemble Prediction Experiments (HEPEX; Schaake et al., 2007) initiative. Datasets such as the ERA40 (Uppala et al., 2005) have been widely used. Although 
these datasets are not originally produced over sets of grids, or at least not the commonly recognised types of grids; they often are interpolated onto regular grids nevertheless in order to facilitate further analysis and for being used as other model inputs. For instance, global numerical weather models tend to use the Gaussian Grids, e.g., EAR40 grids. The local area model (LAM), such as the Weather Research and Forecasting model (WPF; Skamarock et al., 2001) uses regular grids spatially but does so only on a projected plane. Moreover, the importance and hence popularity of using these grid-based forcing data are also underlined by the needs of many climate change impact studies where climate projections, such as those from the Coupled Model Inter-comparison Project (Covey et al., 2003; Giorgetta et al., 2013), are normally provided over a set of regular longitude/latitude grids over the globe. To better facilitate the community in using these grid-based data and encourage the interoperability among models, the Network Common Data Format (NETCDF; Rew and Davis, 1990) has become the de-facto standard in climate change impact studies, although other traditional formats such as GRIdded Binary (GRIB; Rutledge et al., 2006) or Hierarchical Data Format (HDF; Duane et al., 2000) are well supported as well. And some open-sourced libraries such as Geospatial Data Abstraction Library (GDAL) released by the Open Source Geospatial Foundation (Warmerdam, 2008) have been developed and published openly for reading and writing various types of geospatial data formats (e.g., at least 154 raster and 93 vector data formats can be supported by GDAL) and applied in many areas of science (Zhan and Qin, 2012).

However, applications of these new datasets also bring some challenges. For example, in the context of using grid-based hydroclimatic datasets for providing external forcing field of hydroclimatic extreme quantification, an important step is to understand, quantify and if possible, correct the errors and/or bias in these fields. The spatially variant nature of these data remains the centre of the process. Some efforts have been put where e.g., Rojas et al. (2011) applied a statistical bias correction to improve the regional climate model (RCM) driven climate simulations across Europe; Rabiei and Haberlandt (2015) proposed to merge rain gauge measurements and weather radar data which is grid-based data by bias correction. Specifically for weather radar adjustment, many algorithms such as the Mean Field Bias (MFB) method 
and the Kriging with External Drift (KED) method, adjust the radar data solely by a multiplicative factor which does not vary spatially; however, more recently the Conditional Merge algorithm introduced by Sinclair and Pegram (2005) and implemented by Guenzi et al. (2017), considers the spatial impacts by conditioning the gauge adjustment on the radar precipitation values at gauge locations (Silver et al., 2019).

Apart from being used as inputs to the models, the grid-based hydroclimatic datasets are also a foundation to support further analysis on environmental change both spatially and temporally. It is clear from the above examples that the grid-based hydroclimatic data have spatial patterns and characteristics with regard to certain changing factors that need to be diagnosed. Such diagnosis, without exception, is done over analysing targeted variable(s) and/or their combinations sampled spatially within predefined boundaries such as political regions (Bell, 1976) and river catchments (Monteiro et al., 2016). Further, to understand the random nature of the errors and uncertainties associated with the spatial data, the Monte-Carlo simulation approach is commonly used to be combined with geostatistical stochastic simulation for uncertainty quantification. A simple procedure of such is to perform simulations of points (can be data or events) randomly distributed in the predefined area, calculate the empirical distribution function of such inter-point distances in each case and then obtain further values of the statistic by the goodness of fit (Besag and Diggle, 1977). Following this approach, some applications have been published, e.g., Smith and Cheeseman (1986); Xu et al. (2005) and Wu et al. (2018); however, applications on hydroclimatic grid-based data remain scarce and many previous studies on the spatiotemporal analysis of hydroclimatic variables were conducted over predefined areas.

\section{- Spatiotemporal variation analysis of hydroclimatic extremes using grid-based data}

As these new, spatially disaggregated, grid-based hydroclimatic datasets have become more accessible to the research community, it is unsurprising that more and more research has started to make use of these datasets to study the spatiotemporal variation of hydroclimatic events (Peleg et al., 2018), and some are at extreme levels. For example, Du et al. (2019) identified the spatiotemporal variation and trend of precipitation and streamflow extremes in the Xiang 
river basin using gridded data with the horizontal resolution of 0.5 degrees. They analysed the trend of each grid of precipitation extremes at different time scales and revealed the spatial distribution. Fairman Jr et al. (2017) studied the effects of size, shape, and intensity of precipitation features that are defined as contiguous areas of nonzero precipitation rates on the precipitation climatology over Great Britain and Ireland from 2006 to 2015; Laverde-Barajas et al. (2020) proposed a spatiotemporal object-based method and analysed the spatiotemporal characteristics of different storm events at catchment scale (duration, spatial extent, magnitude, and centroid) by using a 3D pattern (e.g., the storm event changes in the scale of longitude, altitude and time) extracted from gauge-adjusted weather radar data. Diaz et al. (2020) proposed a method to monitor the spatial paths of drought (i.e., drought centroids localisation and linkage) and its severity and duration by using grid data from the Standardized Precipitation Evaporation Index (SPEI) Global Drought Monitor over India (1901-2013). And more applications focused on spatial variation of grid-based hydroclimatic observations can be seen in Drusch et al. (2004), Thorndahl et al. (2017), Chen et al. (2015) and UKCIP (Banwell et al., 2018; Lowe et al., 2018; Prein et al., 2017). Most of the abovementioned studies for quantifying or analysing the hydroclimatic extremes varying with time and space, are on a perevent basis and then use different approaches to explore its development in spatial and temporal extent, which is one of the main objectives for spatiotemporal quantitation analysis in hydroclimatic extremes.

Another objective of the research in this area is to attempt to address the spatiotemporal variation of hydroclimatic extremes to support the water infrastructure engineering design, for example, the frequency analysis (Li et al., 2015; Overeem et al., 2010). A classical analysis approach for designing and validating many infrastructure systems (Climate Data, 2009) is to use historical hydroclimatic extreme data, such as maximum rainfall, temperature, river flows etc., to estimate the parameters of the required extreme value model which would offer probability distributions of the natural phenomenon in question, so as to address its occurrence or exceedance probability at given thresholds in a given region of interest. Since Jenkinson (1955) proposed a generalised approach to analyse the frequency distribution of annual maxima, much effort has been made in quantifying the natural phenomena at extreme levels 
by using the Generalised Extreme Value (GEV) models with parameter estimation using the Maximum Likelihood (ML) method and L-Moments (LM) method, especially in designing and planning water engineering systems (Coles and Tawn, 1996; Lazoglou and Anagnostopoulou, 2017; Mannshardt-Shamseldin et al., 2010; Shukla et al., 2012; Yoon et al., 2015). As the parameters are usually assumed to be unchanged, the risk assessment based on frequency analysis is defined as to be stationary.

However, the last few decades have witnessed significant disturbances and changes to the hydrological regimes caused by climate change and human activities, which have resulted in changing frequency and intensity of hydrological extreme events over the world. Numerous studies have researched many key hydroclimatic variables, such as precipitation, temperature, streamflow, as well as the compound events such as extreme flooding related to joint rivertide-storm surge (De Luca et al., 2020; Zscheischler et al., 2018) which are indeed changing due to the impact of climate change (Assani and Guerfi, 2017; Herring et al., 2018). Therefore, in view of the reliability of infrastructure designs based upon extreme value analysis, stationary risk analysis has been re-assessed from a new adaptive perspective where Sarhadi et al. (2016) proposed a multivariate time-varying risk framework for all stochastic multidimensional systems under the influence of a changing environment. For the commonly used nonstationary GEV model, this is meant to assume that its parameters vary with time or other climate covariates. For example, Hasan et al. (2012) proposed two nonstationary GEV models for extreme temperature and each model assumes only one parameter as nonstationary depending linearly and exponentially in time respectively. Sarhadi and Soulis (2017) defined both the scale and location parameters for extreme precipitation analysis using a linear, time-varying representation. Their results demonstrated the underestimation of extreme precipitation if stationary models are used. Panagoulia et al. (2014) generated 16 nonstationary GEV models of extreme precipitation with linear time dependence of location and log-linear time dependence of scale, employing the Akaike Information Criterion (AIC) and the Bayesian Information Criterion (BIC) for selecting the best model, and examined confidence intervals for model parameters. Different from the researches listed above which assume a constant 
shape parameter, Ragulina and Reitan (2017) explored the change of the shape parameter and found that it evidently depends on the altitude of study areas.

In summary, there has been a growing interest and related studies in quantifying the variation of hydroclimatic extremes in space and time, e.g., some focused on the event-based development of hydroclimatic variables and studied its duration, magnitude and spatial extent whilist others involved non-stationarity to fit a long-term record of series of hydroclimatic extremes (e.g., annual/seasonal/monthly maximum daily precipitation or streamflow) and then attempted to explore its spatial distribution. However, a limited number of specific domains or events were used because of data availability issues in hydrological observations somehow affect the outcome of studies e.g., the lack of generalisation over space (Ganguli and Coulibaly, 2017), therefore, study of spatiotemporal variation of hydroclimatic extremes still has a long way to go.

\subsubsection{Climate modes of variability and hydroclimatic extremes}

Many hydroclimatic extremes result from natural climate variability (Dadson et al., 2019). In the hydroclimatic extreme study, the climate modes of variability are important for discussion, which aims to explain how climate variability triggers the changes of extremes (Field et al., 2012; Seneviratne et al., 2012).

In recent decades, many efforts have been made in identifying and quantifying the link between extreme events and possible natural climate modes such as El Niño-Southern Oscillation (ENSO), North Atlantic Oscillation (NAO), the Indian Ocean Dipole (IOD), Arctic-midlatitude interactions, sea surface temperature (SST), anthropogenic mode and land-surface process. For example, Scaife et al. (2008) estimated the changes of both temperature and precipitation in extreme winter weather events over Europe based on the historical observation of 30 years and found that large changes in the frequency of $10^{\text {th }}$ percentile temperature and $90^{\text {th }}$ percentile precipitation events are attributed to the changes of NAO on regional and seasonal scales. Zhao et al. (2016) quantified the contributions of anthropogenic factors including the greenhouse gases (GHGs), anthropogenic aerosols (AAs), and land use (LU) and external natural forcing 
to climate change in China by using both observed temperature and precipitation records and simulations from the Coupled Model Intercomparison Project Phase 5 archive (CMIP5) over 50 years. They found that both the anthropogenic (GHGs and AAs) and external natural forcing contribute to a very high percentage (around 95\%) of the changes of observed temperature; and both LU and external natural forcing contribute around $70 \%$ of changes on the long-term nonlinear trends in precipitation.

Among all the climate modes that lead to the variability of hydroclimate extremes, the El NiñoSouthern Oscillation (ENSO) has been regarded as one of the most profound climate phenomena related to hydroclimate extremes in the tropical Pacific, which has a global effect. An El Niño episode is usually associated with abnormal/different frequencies of occurrence of climate extremes such as very heavy rainfall and extreme temperatures, which brings challenges for organizations coping with natural disasters. And the climate variability induced by El Niño is worldwide and its impacts have been observed in many regions, e.g., North America (Larkin and Harrison, 2005; Neale et al., 2013; Shimizu et al., 2017), Western Pacific and Indian Oceans (Ratnam et al., 2014; Yadav et al., 2013), East Asia (Jiang et al., 2019; Liu et al., 2014), Europe and the Atlantic Ocean (Amaya and Foltz, 2014; Graf and Zanchettin, 2012), and Arctic (Hu et al., 2016; Kim et al., 2020). However, an El Niño episode is difficult to predict because of its diverse properties, e.g., El Niño can sometimes be observed in the eastern Pacific and sometimes occur in the centre and sometimes in both portions of the Pacific simultaneously. In other words, the generation mechanisms of different ENSO events may not be the same, which cannot be fully described by one single index. By tracking the central location of the ENSO-associated SST anomalies, Trenberth and Stepaniak (2001) proposed that the different types of ENSO can be contrasted by differentiating the ENSO-associated SST anomalies between the central and eastern Pacific. Therefore, the different generation mechanisms and underlying dynamics of two distinct types of ENSO have been identified (Kim and Yu, 2012; Ashok et al., 2007; Fedorov et al., 2015; Yu and Kim, 2010), i.e., a CentralPacific type located in the central tropical Pacific and less sensitive to thermocline variations during its generation and an Eastern-Pacific type located mainly in the tropical eastern Pacific and its generation involves equatorial thermocline variations. And in recent two decades, there 
is a growing interest in studying different impacts produced by these two types Central-Pacific El Niño and Eastern-Pacific El Niño and their teleconnection associated with the change of hydroclimate extremes.

In summary, these climate modes of variability, which span a range of time and space scales, can greatly help risk managers to understand the physical mechanisms and variability of the extreme phenomenon in hydrology and meteorology.

\subsubsection{Climate models, climate projections and hydroclimatic extremes}

Another strand of research to quantify the possible changes in hydroclimatic extremes driven by climate change has concentrated on the provision of projections of the relevant meteorological components of the water balance in the future (Dadson et al., 2019). Many climate models (CM) are well established for simulating climate dynamics and forecasting the future. A commonly applied method is to use the outputs of general circulation models (GCMs; Colman, 2003) as the driving boundary conditions, simulating various hydroclimatic variables, such as the changes of rainfall, flood and groundwater etc, at a specific scale by using regional climate models (Im et al., 2015; Sato et al., 2007). The GCMs are the basic tools for simulating global changes due to greenhouse gas emissions which lead to the changes of hydroclimatic variables under the effects of climate change on a global scale. However, it is widely recognised that all climate models can contain various uncertainties coming from, e.g., the assumption of initial conditions of the atmosphere and ocean, the dynamic and physical formulation of the model structure and the scenarios of economic activity on which the models are based. Thus, how to reduce/quantify uncertainties thereby improving the accuracy of climate simulation and projection is the main issue to be addressed (Weigel et al., 2010; Weigel et al., 2008).

A typical approach is to combine and upgrade the CP models, e.g., using ensemble/coupled climate simulations with various combinations of RCMs and GCMs that can provide a better simulation under climate change within an uncertainty range. It exploits the strengths of diverse methods or perspectives and yields a more appropriate approach to reduce the simulation errors and increase the accuracy of climate prediction, which is currently in use as a matter of routine 
within national meteorological services (Buontempo et al., 2015; Meehl et al., 2007; Randall et al., 2007).

Moreover, in multi-model climate model projection developments, the Coupled Model Intercomparison Project (CMIP) is one of the well-known foundational frameworks of climate science used for the design and distribution of global climate model simulations of the past, current, and future. The CMIP project has evolved over six phases and has been applied to various international multi-model research (Eyring et al., 2016; Waliser et al., 2020). Besides, it remains an authentic data source for producing national and international climate-change assessment and impact studies by making the outputs available in a standardised format for public use.

In recent decades, many efforts have been made to evaluate the skills of CMIP and to improve the climate simulations. For example, Ramirez-Villegas et al. (2013) assessed 24 CMIP3 and 26 CMIP5 models of their simulation skills by studying the mean climate and interannual variability for four seasons in five tropical regions. They found that the simulation on seasonal precipitation and wet-day frequency depicts a larger error than seasonal mean temperature and in more than $30 \%$ of the study areas, no single GCM matches observations for monthly precipitation and wet-day frequency, $50 \%$ for diurnal range and $70 \%$ for mean temperatures. Comparing the generations of CMIP, Cannon (2020) found that the latest generation (i.e., CMIP6) of climate models provide less biased simulations for use in regional dynamical and statistical downscaling efforts than previous generations by comparing historical simulations of daily sea-level pressure circulation types over 6 continental-scale regions (North America, South America, Europe, Africa, East Asia, and Australasia) by 15 pairs of global climate models from CMIP5 and CMIP6. Di Luca et al. (2020) explored and quantified the skill of CMIP5 and CMIP6 models for simulating daily temperature extremes by composing the temperature extremes errors and found that although CMIP models systematically exaggerate the magnitude of daily temperature anomalies for both cold and hot extremes, they can simulate temperature extremes well. Besides, the CMIP6 improvements relative to CMIP5 are systematic across most land areas and are only partially explained by the increase in horizontal resolution and other differences must therefore help explain the higher CMIP6 skill. Some 
other studies also have been carried out to explore the gap between the latest CMIP6 with previous generations when simulating variables such as precipitation, sea levels and temperature in different regions, e.g., Séférian et al. (2020); Xin et al. (2020); Gusain et al. (2020); Stouffer et al. (2017).

Another approach is to use the downscaling technique to increase the accuracy of simulation as the horizontal grid spacing of most global climate models is widely recognised as too coarse to adapt to the local-scale decisions (Dadson et al., 2019; Maraun et al., 2010). Normally, downscaling can be done statistically by either using the empirical relationship between predictors in a global model and variables of interest, or dynamically using a regional model with a higher resolution within a limited area which is a subdomain of the global model. For instance, due to the limitations in resolution and physical parameterisations of GCMs, e.g., the typical horizontal resolution of GCM is 250 to $600 \mathrm{~km}$ (IPCC., 2021), in order to simulate the hydroclimate variables at smaller scales such as a regional or local scale, the dynamical downscaling (DDS; Xu et al., 2019) technique has been proposed and applied to estimate various climatic conditions with a fine/higher resolution (such as $\sim 50 \mathrm{~km}, \sim 25 \mathrm{~km}$ and even $\sim 10 \mathrm{~km}$ ) in a physical model by involving detailed geographic information such as topography. It can be carried out by the regional climate model (RCM) nested within GCM simulations.

Although the performance of climate projection simulations has been remarkably improved with even higher resolutions and now can be accessed by the public, the improvements are not enough nor effective due to technology limitations, knowledge level of nature, etc, in many respects and especially in terms of revealing extreme events. Most abovementioned studies treated the climate as a mean level of the state. How to bridge the gap between the climatechange data produced by climate projections and the in-situ observations for hydro-climatic extremes, has yet to be explored fully. 


\subsubsection{Computer vision and its potential application in recognising and classifying hydroclimatic extremes}

Computer vision is an interdisciplinary field of science that aims to produce digital systems for processing, analysing, and understanding visual data such as images and videos at a high level in the same way that the human visual system does (Huang, 1996). It is not a new technology since the historical roots of computer vision began in the 1950s (Rosenfeld, 1998). The early foundations of computer vision have been constructed in the 1970s with many algorithms are proposed e.g., extraction of edges, labelling of lines, non-polyhedral and polyhedral modelling, representation of objects as interconnections of smaller structures, optical flow, and motion estimation (Baumgart, 1974; Milgram, 1979; Shirai, 1979; Zucker, 1976). Then the studies turned to focus on more rigorous mathematical techniques for performing quantitative image and scene analysis to deduce the shape by studying shading, texture and contour (HG Barrow et al., 1978; Harry Barrow et al., 1978; Rosenfeld and Weszka, 1980; Terzopoulos, 1983). In the 1990s, a lot of efforts were made on projective 3-D reconstructions for recognition, e.g., multi-view stereo algorithms can produce a complete 3-D surface (Szeliski, 1999). In this period, many algorithms/techniques have been improved, such as tracking algorithms (Guo and Ljung, 1995; Kass et al., 1988), optical flow methods (Bergen et al., 1992; Bruhn et al., 2005), image segmentation (Pavlidis and Liow, 1990; Shi and Malik, 2000), factorization techniques (Poelman and Kanade, 1997). Especially, statistical learning techniques emerged and were firstly applied in practice to recognise faces from images and it should be highlighted that the interaction between computer vision and computer graphics was increased significantly during this period (Szeliski, 2010), e.g., in the field of image-based rendering and modelling (Shum et al., 2008), image morphing techniques (Wolberg, 1998), view interpolation (Chen and Williams, 1993), panoramic image stitching (Chen and Klette, 1999) and full light-field rendering (Levoy and Hanrahan, 1996). The 2000s has continued to witness a deepening interaction between computer vision and graphics and some notable techniques such as computational photography technique that can create and convert the high dynamic images to displayable results and extract different regions from even the overlapping images (Agarwala et al., 2004), feature-based recognition technique (Betta et al., 2011), Markov random field 
(MRF) in reference algorithm (Mehta et al., 2018). Recent progress has been seen in the application of machine learning techniques to computer vision problems. Thanks to the rapid development of new hardware paired with these sophisticated algorithms, computer vision techniques can process more complex problems of identifying, recognising and tracking the patterns from the complicated world. Nowadays, computer vision has been applied in various fields e.g., bioinformatics, signal processing, image analysis, information retrieval, robotic navigation (Paolanti and Frontoni, 2020; Stowell and Plumbley, 2014).

In the field of hydrology and climate science, computer vision has been applied in some research such as flood monitoring and mapping (Arshad et al., 2019), fusing spatiotemporal data for hydrological modelling (Jiang et al., 2018), and causal inference for climate change events from satellite images (Ramachandra, 2019). In particular, the last decade witnessed a dominating trend of applying machine/deep learning techniques to computer vision, for solving hydroclimatic pattern recognition problems due to the increased availability of big data (Bishop, 2006). For example, recently, some researchers applied machine learning methods such as artificial neural networks (ANNs) to extract features of spatiotemporal climatic variables (Qiu et al., 2017; $\mathrm{Wu}$ et al., 2013); others parameterised those features (Gentine et al., 2018; O'Gorman and Dwyer, 2018) to realise weather predictions (Liu et al., 2016; Petersik and Dijkstra, 2020). Among them, Nayak and Ghosh (2013) developed an algorithm by incorporating a machine learning technique based on a support vector machine (SVM), to identify the specific patterns before extreme events (a lead time of $6 \mathrm{~h}$ to $48 \mathrm{~h}$ ) therefore predicting the extreme rainfall in Mumbai, India, using mesoscale and synoptic-scale weather patterns. They introduced two phases of support vector classifier as the significant differences that were observed between the weather patterns before the extreme rainfall during night-time and day-time and used frequency of high anomaly values of weather variables at different pressure for the predictors of SVM. They also compared the performance of the prediction with the state-of-the-art statistical technique fingerprinting approach and found a better prediction by the SVM algorithm.

Nguyen-Le and Yamada (2019) used Self-Organizing Maps (SOM) combined with the Kmeans clustering technique to classify the anomalous weather patterns (WPs) of heavy rainfall 
days during the summertime (May-June, MJ, and July-August-September, JAS) in the year of 1979 to 2007 over the Upper Nan River basin, Thailand. Their results revealed that the primary factors for producing local heavy rainfall are the westerly summer monsoon in MJ and westward-propagating tropical disturbances including tropical cyclones in JAS. They applied the classification results to predict the occurrence of heavy rainfall days over the basin in summer 2008-2017 using prognostic WPs from the operational Japan Meteorological Agency Global Spectral Model (GSM) and found a significant improvement to the forecast skills up to 3-to-33-day lead times.

Chattopadhyay et al. (2020) applied an unsupervised clustering algorithm to classify the daily large-scale weather patterns over North America and then evaluated the performance of the deep learning method convolutional neural network (CNN) to re-identify and predict these clusters up to 5 days ahead of time. The results showed that CNN that identified 1000 samples or more per cluster has an accuracy of $90 \%$ or better for both identification and prediction while the prediction accuracy scales weakly with the number of lead days and CNN outperforms logistic regression, a simpler machine learning algorithm.

Although there have been a few studies attempting to incorporate machine/deep learning techniques into pattern recognition for classifying and predicting extreme events in hydroclimatic science, there is still a challenge needing to be addressed due to the properties of climate and environmental data. These data provided either by model simulations or via observations are usually spatiotemporal, nonlinear, nonstationary, chaotic with high dimensions and large scales. For example, large-scale atmospheric circulation significantly can affect the daily weather and extreme events, which leads to coherent and correlated patterns due to various physical processes, and non-stationarity due to coupling and anthropogenic effects. In addition, the observational datasets are usually not long enough for training the required algorithm, in addition to the persistent noise in measurements. Therefore, the robustness of pattern recognition has not been fully studied yet. 


\subsection{Research objective and questions}

According to Section 1.2, the gaps can be identified as:

- As more grid-based hydroclimatic data become available, their spatial patterns and characteristics concerning possible factors need to be diagnosed. Meanwhile, the substantial overhead of computing these large-sized datasets has affected research's capacity of studying the spatiotemporal variation of hydroclimatic extremes, especially in the view of non-stationarity. Therefore more efficient sampling tools and methods are worthy to be explored.

- Recent spatiotemporal quantification analysis mostly focused on the hydroclimatic extremes on the per-event basis and attempted to study its boundary and spatial development. However, corresponding to the common practice where extremes for given areas of concern, the spatial features of the regions of interest (ROIs) play a crucial role in determining the amount of quantity of hydroclimatic extremes.

- Many climate projection models and related quantification studies have shown a strong ability to capture the average level of hydroclimatic variables under climate change. Although some studies focused on the extreme level, they were frustrated by the limited length of data, the small number of study areas thereby lacking generalisation.

- Computer vision algorithms have been developed and used widely in many areas of science, however, their techniques and applications in studying climate change and quantifying hydroclimatic extremes is a challenge due to the properties of the environmental data which are usually interrelated, chaos and large-scaled.

To attempt to fill these gaps, three objectives are proposed: the first objective of the study is to develop a quantitative modelling framework that is expected to be widely used for quantifying spatial and temporal variation of hydroclimatic extremes. Unlike the event-based analysis, the framework is further to support an ROI-based analysis that focuses on certain hydroclimatic variables in a number of regions with different locations, sizes, shapes and orientations and studies how the variability of extremes in time (by involving the perspective of non- 
stationarity) and space can be related/sensitive to different features of regions. The potential of the proposed quantitative framework is to be illustrated using two century-long grid-based datasets of daily rainfall over Great Britain and Australia, as an example. As one of the most representative hydroclimatic variables, rainfall is a dominant component of the global hydrological cycle; intense rainfall can lead to floods. Although the rainfall process is a boundless phenomenon, its area-oriented variation is of the concern of the engineers and flood risk managers. It is clear that the area-oriented rainfall variation and distribution are closely related to the climate at large scale (Millán et al., 2005); in the meantime, local features and processes, such as the topography, urbanisation, as well as the orientation and the size of the area can also affect the rainfall amount in question (Buytaert et al., 2006).

The framework is also extended to estimate the joint probability of compound events driven by more than one hydroclimatic variable in the view of nonstationarity. As very few studies in the field of nonstationary multivariate probability analysis, this context should be highlighted as one of the innovations of the study.

Further activity of applying this quantitative framework is to use the simulated rainfall from two climate models, then compare the variability of rainfall extremes with the observation, aiming to explore the bias of climate projections.

Different from the first objective of ROI-based analysis, the second objective of the study is to attempt to design a more stable algorithm for supporting AI-based training to help classify and monitor the development of rainfall events (or rainfall cells over the threshold) from the largesized, chaos environmental datasets. This algorithm is further tested by three case studies and as the front-end of the deep learning technique, its potential development to support deep learning is also involved.

The third objective is to support decision making under uncertainty in the context of flood forecast and early warning, by evaluating the risk attitudes of decision-makers under uncertain consequences thereby supporting decision making.

Seven research questions are therefore raised for realizing each objective and are specified as: 
Q1 How to sample the large-sized, grid-based hydroclimatic data to generate ROIs and extract the spatiotemporal features from them?

Q2 How do hydroclimatic extremes (rainfall in the study) change over time and space and further affect the spatial dependencies?

Q3 How are the quantitative patterns and variations of rainfall extremes linked to largescale climatology?

Q4 What are the methods for assessing the applicability of quantitative (e.g., stationary or nonstationary) models?

Q5 How to quantify the relationship between observed and climate projected rainfall extremes?

Q6 How to support pattern recognition and classification by applying deep learning techniques effectively and robustly?

Q7 How to understand/represent the decision-making process when providing uncertain information of hydroclimatic extremes such as flood forecasts?

\subsection{Study datasets}

There are four grid-based datasets used in this study, two of which are observed rainfall records named as the "Gridded Estimates of daily Areal Rainfall" (GEAR; Tanguy et al., 2016) and the "Australian Data Archive for Meteorology" (ADAM; Jones et al., 2009). The rest are simulated rainfall datasets from climate model projections, which are the "ERA20CM" (Hersbach et al., 2015) and "UKCP18" (Lowe et al., 2018). The details are shown in Table 1.1.

Table 1.1 The datasets applied in the study.

\begin{tabular}{|c|c|c|}
\hline Types & Observation & Simulation \\
\hline
\end{tabular}




\begin{tabular}{|c|c|c|c|c|}
\hline $\begin{array}{c}\text { Name of } \\
\text { Dataset }\end{array}$ & GEAR & ADAM & ERA20CM $^{1}$ & UKCP1 $8^{2}$ \\
\hline $\begin{array}{l}\text { Country } \\
\text { Covered } \\
\end{array}$ & $\begin{array}{c}\text { Great Britain } \\
(\mathrm{GB})\end{array}$ & Australia (AU) & $\begin{array}{c}\text { Great Britain } \\
(\mathrm{GB})\end{array}$ & $\begin{array}{c}\text { Great Britain } \\
(\mathrm{GB})\end{array}$ \\
\hline $\begin{array}{c}\text { Temporal } \\
\text { Resolution }\end{array}$ & 24 hours & 24 hours & 3 hours & 24 hours \\
\hline $\begin{array}{c}\text { Spatial } \\
\text { Resolution }\end{array}$ & $1 \mathrm{~km} \times 1 \mathrm{~km}$ & $\begin{array}{c}0.05^{\circ} \times 0.05^{\circ} \\
(\text { approx. } 5 \mathrm{~km} \times \\
5 \mathrm{~km})\end{array}$ & $0.4^{\mathrm{o}} \times 3.15^{\mathrm{o}}$ & $\begin{array}{c}2.2 \mathrm{~km} \times 2.2 \\
\mathrm{~km}\end{array}$ \\
\hline $\begin{array}{l}\text { Overall } \\
\text { Covered } \\
\text { Area }\end{array}$ & $700 \times 1250 \mathrm{~km}^{2}$ & $\begin{array}{l}44.5 \mathrm{~S}-9.95 \mathrm{~S} \\
112 \mathrm{E}-156.3 \mathrm{E}\end{array}$ & Whole UK & Whole UK \\
\hline Duration & $\begin{array}{c}1898-2010 \\
(113 \text { years })\end{array}$ & $\begin{array}{c}1890-2018 \\
(129 \text { years })\end{array}$ & $\begin{array}{c}1900-2010 \\
(111 \text { years })\end{array}$ & $\begin{array}{c}1981-2000 \\
(20 \text { years })\end{array}$ \\
\hline Provider(s) & $\begin{array}{c}\text { Centre for } \\
\text { Ecology \& } \\
\text { Hydrology }(\mathrm{CEH})\end{array}$ & $\begin{array}{l}\text { The Bureau of } \\
\text { Meteorology }\end{array}$ & $\begin{array}{c}\text { European } \\
\text { Centre for } \\
\text { Medium-Range } \\
\text { Weather } \\
\text { Forecasts } \\
\text { (ECMWF) }\end{array}$ & $\begin{array}{c}\text { Met Office } \\
\text { Hadley Centre }\end{array}$ \\
\hline
\end{tabular}

The GEAR dataset is a grid-based $\left(1 \times 1 \mathrm{~km}^{2}\right)$ rainfall estimation that covers the mainland of Great Britain (GB) from 1898 to 2010. It is derived from the UK Met Office national database of observed precipitation from the UK rain gauge network. The natural neighbour interpolation method with a normalisation step based on the average annual rainfall, was used to generate the daily estimates (Tanguy et al., 2016). The geographical origin of the GEAR data matrix starts from the location of $400 \mathrm{~km}$ west, $100 \mathrm{~km}$ north of the true Origin $\left(49^{\circ} \mathrm{N}, 2^{\circ} \mathrm{W}\right.$, an offshore point in the English Channel which lies between the island of Jersey and the French port of St. Malo), spreading $700 \mathrm{~km}$ eastward and $1250 \mathrm{~km}$ northward. The coordinates are in the National Grid Reference (NGR; Ordnance Survey, 1946) which is a projected map coordinate system with the easting $(x-)$ and northing $(y-)$ expressed in linear kilometres.

\footnotetext{
${ }^{1}$ To match the spatial and temporal resolutions of the ERA20CM dataset with the GEAR dataset, I firstly project the longitude/latitude coordinate of the ERA20CM dataset to the NGR by adopting the transverse Mercator projection method, then convert the 3-hour rainfall to the 24-hour-based ( 9 am- 9 am) by statistical aggregation.

${ }^{2}$ To match the spatial and temporal resolutions of the UKCP18 dataset with the GEAR dataset, I firstly rotate the coordinate back to be the same with the standard reference NGR and do $1 \mathrm{~km}$ interpolation to keep the same size of grids.
} 
The ADAM dataset is generated using a sophisticated analysis technique described in Jones et al. (2009), which is also grid-based $\left(0.05^{\circ} \times 0.05^{\circ}\right.$, approx. $\left.5 \times 5 \mathrm{~km}^{2}\right)$ rainfall from $01 / 01 / 1900$ to 31/12/2018 over Australia (AU) based on the Geocentric Datum of Australia 1994 (GDA94; Collier, 2002) with the origin $\left(44^{\circ} \mathrm{S}, 112^{\circ} \mathrm{E}\right)$ i.e., $(0,0) \mathrm{km}$, and easting $(x$ - $)$ and northing $(y$ - $)$ transformed to kilometres.

The recorded rainfall values of both observed datasets are provided as daily rainfall, i.e., the total rainfall amount over a predefined 24-hour ( 9 am-9 am) period which refers to the 24 hours prior to the reporting time for the ADAM dataset and the 24 hours after for the GEAR dataset.

The ERA20CM data is derived from an ensemble climatic projection (with 10 ensemble members) at a $0.4^{\circ} \times 3.15^{\circ}$ (latitude by longitude) with a 3-hour spatial and temporal resolution from 1900 to 2010 (Hersbach et al., 2015), provided by the European Centre for MediumRange Weather Forecasts (ECMWF).

The UKCP18 (short for 'the UK Climate Projection 2018 project') data are produced by the UK Met Office Hadley Centre, providing changes in various climate variables over the GB in rotated pole coordinates, downscaled to a high spatial resolution $(2.2 \mathrm{~km})$. The entire projections have three time-slices (1981-2000, 2021-2040 and 2061-2080), for a high emissions scenario, RCP8.5. In this research, I selected the first time-slice $2.2 \mathrm{~km} \times 2.2 \mathrm{~km}$ grid-based daily rainfall data compared with the GEAR data which are observed rainfall.

\subsection{Layout of thesis}

This thesis is structured with nine chapters which are further organised into five parts: the introduction, conclusion and the three main technical parts which correspond to the three objectives proposed in Section 1.3. The structure is depicted in Figure 1.1 and described below:

Introduction presents the research background, current literature, objectives and research questions and datasets which are applied in the study (Chapter 1). 
Part $\mathbf{I}$ is the most comprehensive part that discusses the quantitative modelling framework for analysing spatiotemporal extreme patterns, including the development of an important toolbox of spatial random sampling (SRS-GDA) to support the spatial analysis for large-sized, gridbased data (Chapter 2); quantifying the extremes changing over space (geographical location, sampling size and orientation) and time using the proposed probability models framed as the stationary and nonstationary and generalised linear models with the assistance of highperformance computation (HPC) as presented in Chapter 3 and Chapter 4. This framework is further examined by using two series of datasets coming from two types of climate projection models, aiming to reveal its bias on quantifying extremes compared with observations (Chapter 5). Moreover, the framework is extended to estimate the joint probability of the multivariate extremes and a case study for evaluating the risk of compound flood is then applied, which is presented in Chapter 6.

Part II presents the development of a spatial pattern recognition toolbox (SPER) that is used to quantify and extract both spatial and physical features of the hydroclimatic patterns over the threshold. Its applicability and potential ability for supporting AI-based training (such as a convolution neural network) are discussed (Chapter 7).

Part III discusses the building and testing of the two decision-making models driven by decision theory and data respectively to simulate human behaviour in the context of flood forecasting and early warning system. The risk attitudes confronting extreme events are analysed, which contributes to the understanding of the decision-making under uncertainty (Chapter 8).

Conclusion highlights the innovation and contribution of the thesis and discusses the future work (Chapter 9). 


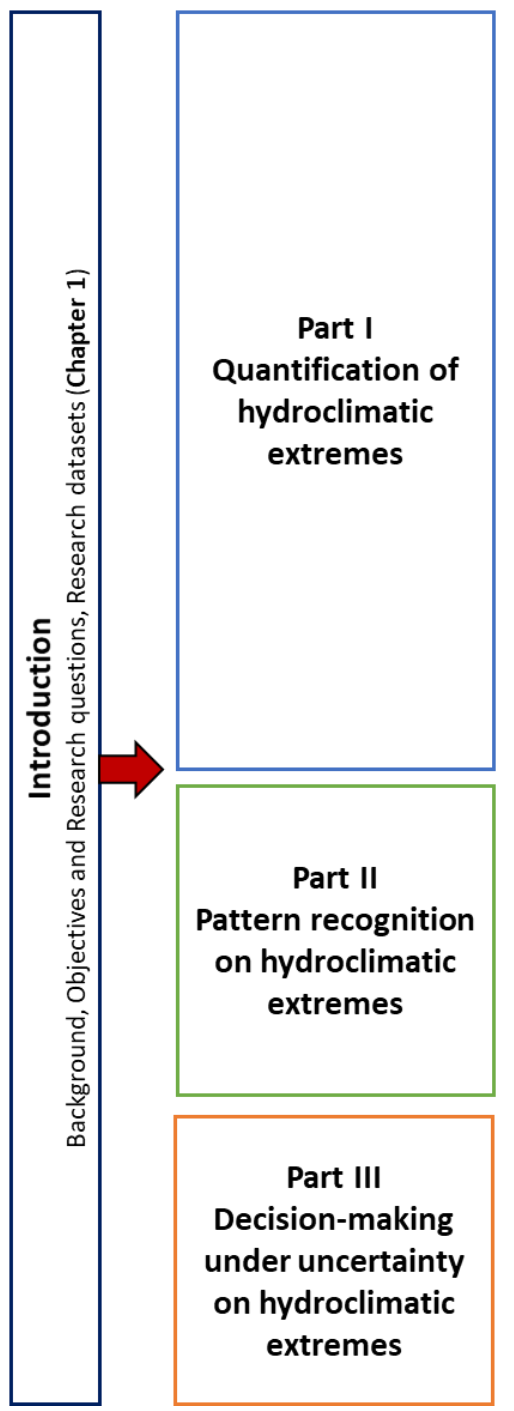

\begin{tabular}{|c|c|}
\hline State of the art & \\
\hline $\begin{array}{l}\text { - Datasets: GEAR, ADAM and EAR2OCM, UKCP18; } \\
\text { - Scope of current research (section 1.2.1, 1.2.2, 1.2.3) } \\
\text { Challenges (section 2.1, 3.1, 4.1, 5.1, 6.1). }\end{array}$ & \\
\hline $\begin{array}{l}\text { Spatial sampling toolbox } \\
\text { - Design a toolbox of spatial random sampling for grid-based data analysis (SRS- } \\
\text { GDA) to generate a great number of regions of interest (ROI) for the } \\
\text { quantification analysis. } \\
\text { (Chapter 2) }\end{array}$ & \\
\hline Quantification on univariate extremes & \\
\hline $\begin{array}{lc}\text { - Quantification on spatial variation of extremes } & \text { (Chapter 3) } \\
\text { - Quantification on temporal variation of extremes } & \text { (Chapter 4) } \\
\text { - Quantification of bias between observed and climate projected extremes } \\
\text { (Chapter 5) }\end{array}$ & 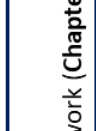 \\
\hline Quantification on multivariate extremes & \\
\hline $\begin{array}{l}\text { Nonstationary multivariate quantitative framework. } \\
\text { - Compound flooding driven by rainfall and skew surge in Ho-Chi-Minh-City, } \\
\text { Vietnam. } \\
\text { (Chapter 6) }\end{array}$ & 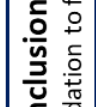 \\
\hline State of the art & ᄃ \\
\hline $\begin{array}{l}\text { - Scope of current research (section 1.2.4); } \\
\text { - Challenges (section 7.1). }\end{array}$ & 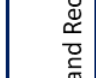 \\
\hline Recognising and classifying hydroclimatic extremes & $\overline{\underline{n}}$ \\
\hline $\begin{array}{l}\text { - Design a toolbox of spatial pattern extraction and recognition (SPER). } \\
\text { - Three illustrative cases and one case for recognising extreme rainfall in UK by } \\
\text { using a convolution neural network. } \\
\text { (Chapter 7) }\end{array}$ & $\begin{array}{l}\frac{J}{U} \\
\frac{0}{0}\end{array}$ \\
\hline State of the art & \\
\hline $\begin{array}{l}\text { - Scope of current research (section 8.1); } \\
\text { - Challenges (section 8.1). }\end{array}$ & \\
\hline Decision-making support and risk attitude analysis & \\
\hline $\begin{array}{l}\text { - Both theory-driven and data-driven models are generated to simulate the } \\
\text { decision in the context of flood forecasting and early warning. } \\
\text { (Chapter 8) }\end{array}$ & \\
\hline
\end{tabular}

Figure 1.1 Layout of the thesis. 


\section{Part I}

Quantitative modelling framework for hydroclimatic extremes 

The quantitative framework has three main steps i.e., sampling (Chapter 2), modelling and quantifying (Chapter 3 and Chapter 4), and is depicted in Figure I.1 where the grid-based rainfall dataset is exemplified.

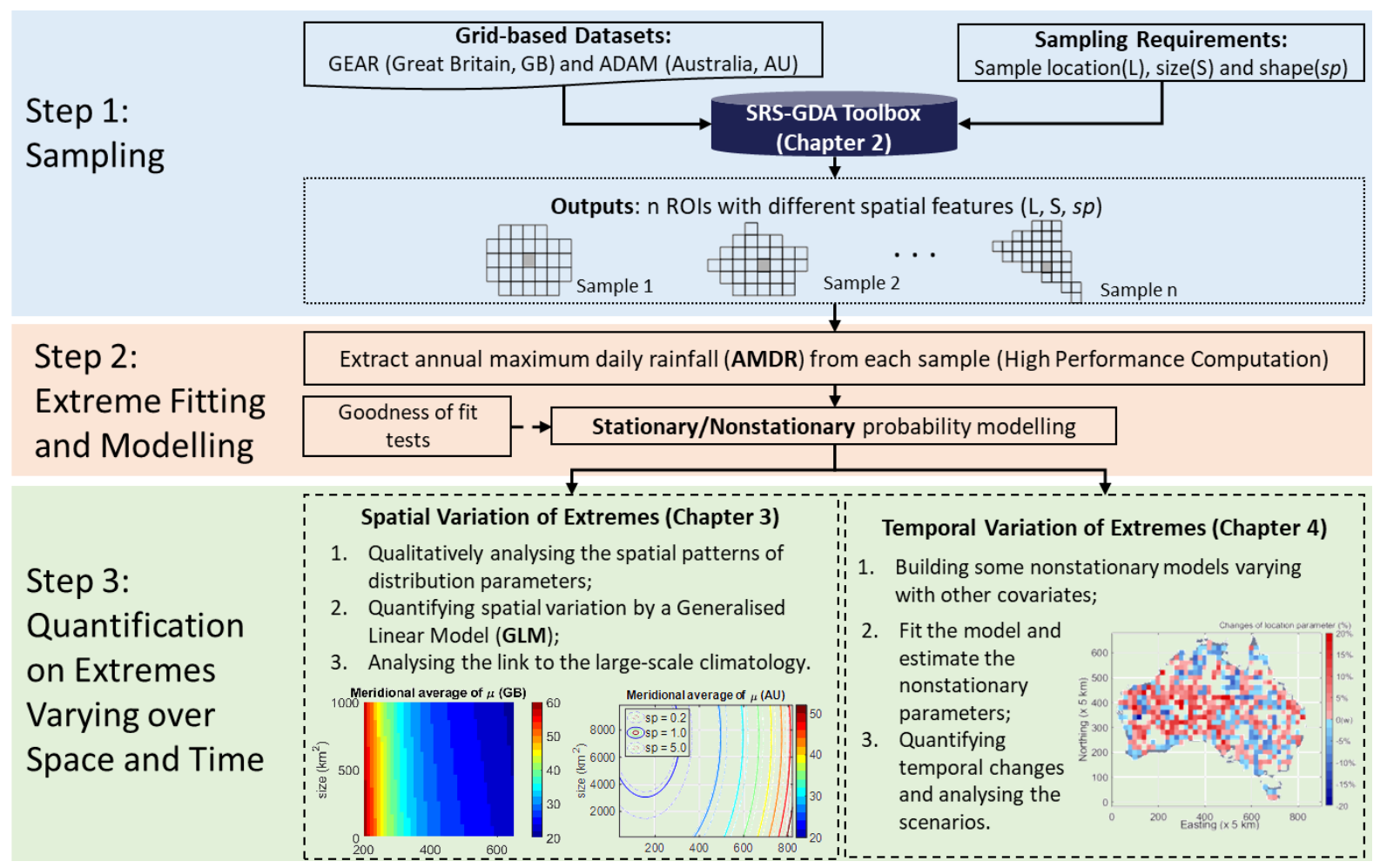

Figure I.1 Quantitative modelling framework for analysing the spatiotemporal variation of hydroclimatic extremes. 



\section{Chapter 2 The Development of a Spatial Random Sampling Toolbox}

Studying hydroclimatic extremes often needs to deal with large-sized, grid-based forcing datasets. How to effectively process such datasets to reveal the patterns and characteristics of hydroclimatic extremes with regard to different spatial features of the regions of interest (ROIs) is the first challenge as discussed in Chapter 1, e.g., as one of the proposed research questions Q1. To address this question and to provide an effective tool for supporting the environmental change studies and my subsequent work (i.e., the ROI-based analysis), a new, open-source SRS-GDA toolbox was developed, aiming to provide a random spatial sampling for grid-based hydroclimatic datasets, which can be used to carry out random spatial sampling of grid-based quantities with various constraints: shape, size, location, dominant orientation and resolution. This toolbox is a fundamental tool to support spatiotemporal analysis on extremes presented in Chapter 3 and Chapter 4.

\subsection{Introduction}

This chapter ${ }^{3}$ presents the development of a Spatial Random Sampling toolbox for Grid-based Data Analysis (SRS-GDA) which can generate arbitrary samples from grid-based environmental observation and modelling dataset automatically, aiming to answer the research

\footnotetext{
${ }^{3}$ Part of the contents of Chapter 2 has been published in "Wang, H., \& Xuan, Y. (2020). SRS-GDA: A spatial random sampling toolbox for grid-based hydro-climatic data analysis in environmental change studies. Environmental Modelling \& Software, 124, 104598. https://doi.org/10.1016/j.envsoft.2019.104598."
} 
question Q1: how to sample the large-sized grid-based hydroclimatic data and extract the spatiotemporal features from them? The main function of this open-source toolbox is highlighted in that it can assist in spatial random sampling with various constraints such as shape, size, location, dominant orientation and resolution which can be regarded as the spatial properties (features) of the data. In the field of environmental change impact studies where the spatial properties of grid-based datasets remain the focus, this toolbox addresses the need of quantifying how hydro-climatic responses vary with location and scale. The grid size of the SRS-GDA toolbox can be defined in line with any resolution of the base grid map. To increase the applicability of this toolbox, users can customise various sampling conditions and their combinations which can be directly applied to many environmental change studies.

This chapter is structured as follows: first, a brief introduction of the study background and the main objective is provided in Section 2.2, followed by the presentation of the methodology section. An example use case of analysing hydro-climatic extremes, i.e., extreme precipitation over Great Britain using the GEAR dataset is provided to demonstrate the application of the toolbox, shown in Section 2.3. Finally, a discussion on further applicability and availability of the toolbox are presented in Section 2.4.

\subsection{The design of SRS-GDA toolbox}

The main aim of designing and implementing the SRS-GDA toolbox is to enable random spatial sampling of grid-based data within a pre-defined Region of Interest (ROI) of different sizes, shapes, locations and resolutions. The sampling procedure starts with a user-supplied grid dataset with spatial reference. It is also common to have an overall boundary (OB) from which the sampling is to be conducted, as many grid datasets have a coverage normally much larger than that of the user's interest, such as the General Circulation Model (GCM) output around the globe. Normally, the OB should be set large enough for studying how the variation of locations can affect certain quantities represented by an ROI. 
The randomisation of the sampling process is manifested by the ways in how the ROI is constructed:

1) Randomisation of the shape of the ROI. The shape of an area often plays an important role in various applications. For example, in hydrology, a so-called donor catchment is often desired to have a shape analogous to that of the ungauged, target one. Understandably, this process sets to be the most complex one in the SRS-GDA toolbox. There are two options are offered with regards to whether the shape of the ROI is concerned: the shape-unconstrained sampling which randomises the shape of ROI; and the shape-constrained sampling that makes use of a predefined geometric shape supplied by the user e.g., a polygon at a given scale. A special case is a point or single grid sampling whose ROI reduces to a single grid. This is also useful, for example, when studying the variation of point-measured quantities.

2) Randomisation of the location of the ROI. The location of an ROI can be varied using the coordinates of its centroid of predefined ROI or its origin for randomly generating ROI. This operation is done by randomly setting a point or grid within the OB as the location of the ROI. An extra step is usually applied to ensure the entire region of the ROI falls within the OB.

3) Randomisation of the size of the ROI. Variation of the ROI size can help users to identify whether the aggregated data value over an area exhibits notable behaviour. A typical case, for example, is to study the extreme value distribution of a hydrometeorological variable - temperature or precipitation, over regions, countries and globally. This operation depends on whether the ROI is shape-constrained or not. If a predefined shape is used, a 'buffering' operation (Chang, 2008) is used to either increase or reduce the size whilst maintaining the shape unchanged; whereas for a shape-unconstrained case, the desired ROI is randomly produced with a given centroid and specified size.

These three operations can be combined to achieve the various levels of randomisation required by users. The implementation of the toolbox involves a series of steps that are described below and shown in Figure 2.1 which includes: (a) Grid map generation which sets the overall 
boundary (OB) spatial coverage constraint and the resolution for the study (sampling) area; (b) Sampling setup that determines whether one or more constraints are used and sets the corresponding values and/or features, for example, location (fixed or floated), shapeunconstrained or shape-constrained, size fixed or not etc. and (c) Sampling processing and validation which are automatically carried out by the SRS-GDA toolbox based on the OB grid map and the constraint setups with extra filters applied to the results depending on extra conditions where appropriate. 


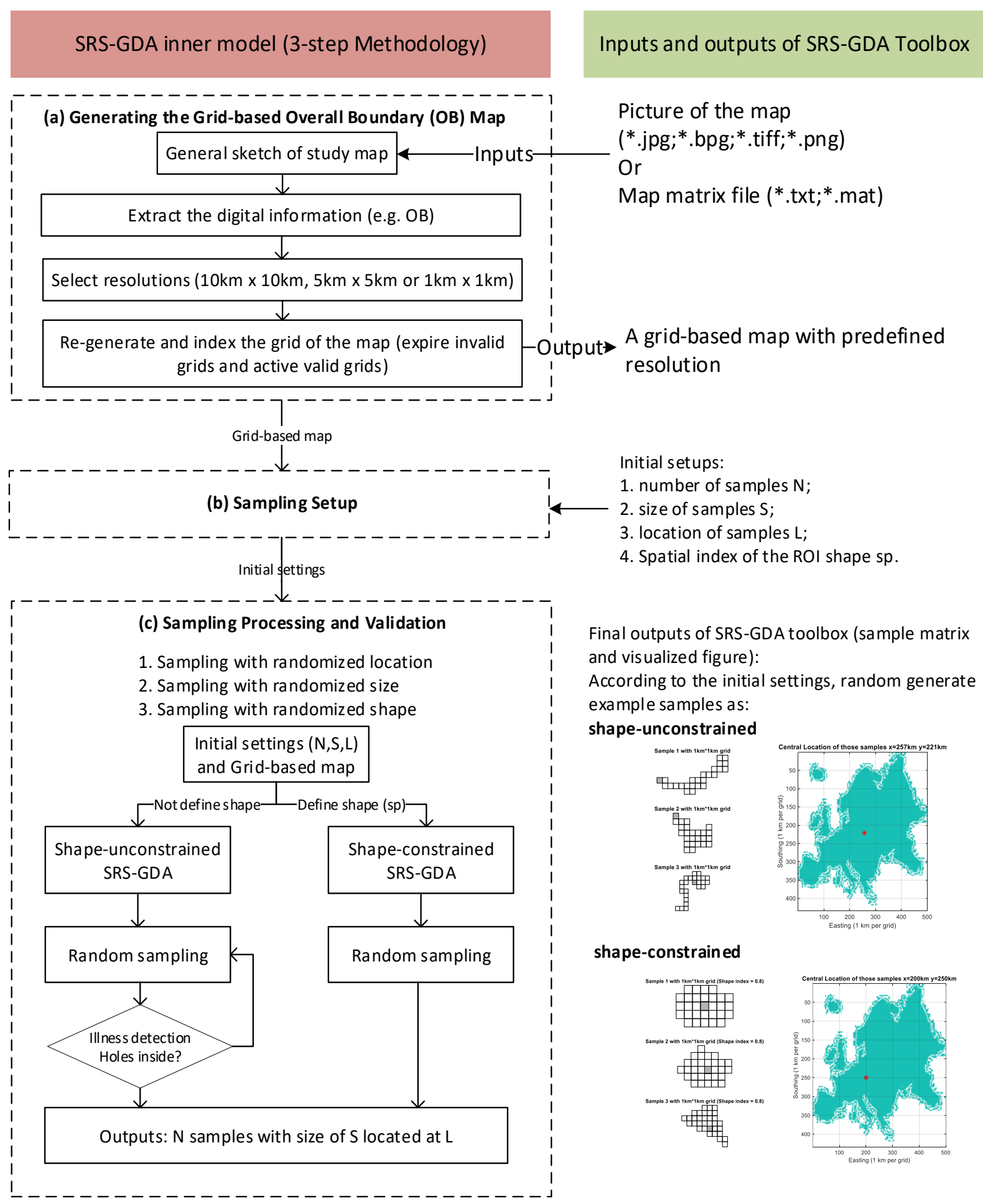

Figure 2.1 The three basic steps and the corresponding inputs and outputs of the SRS-GDA toolbox. 


\subsubsection{Generating the grid-based overall boundary (OB) map}

As mentioned previously, the underlying dataset normally comes with a coverage larger than that of users' interest. In other words, a subset based on an OB needs to be produced. This OB needs to be specified by the user, e.g., by using either a raster file or a vector-based map such as shapefiles that define the boundary. If no OB is specified, the entire coverage of the underlying grid dataset will be used to conduct the sampling process. It should be mentioned that the sampling process is often carried out inside the OB. However, unlike OB, the boundaries specified by the ROI's are deemed to be restrictive and arbitrary as far as a natural process is concerned, such as rainfall and wind speed. The logic behind sampling ROI in OB is because many times only the quantity of certain hydro-climatic variables falling in such given boundary is of concern, for example, rainfall over the urban area of a city is a key element for urban drainage design.

A grid-based map is then generated by rasterising the OB (if it comes as a vector map) using the same projection and grid resolution as the underlying dataset. The grids inside OB are regarded as valid grids while those outside OB are invalid grids. Once this is completed, the toolbox will automatically exclude those invalid grids and activate the valid grids. For example, in the example case given in this paper, the National Grid Reference (NGR, Ordnance Survey, 1946) is used to refer to the coordinates of the grids of the GEAR dataset. The base map is processed to distinguish ocean (so-called invalid grids outside the GB boundary) and land (socalled valid grids inside the GB boundary). It is also further refined to have several versions with different spatial resolutions which are normally multiples (exact divisions) of the grid size of the underlying dataset. These refined OB's will be used for further study on aggregation (upscaling) and disaggregation (downscaling). The toolbox provides three resolutions to match the underlying grid dataset: $1 \mathrm{~km} \times 1 \mathrm{~km}, 5 \mathrm{~km} \times 5 \mathrm{~km}$ and $10 \mathrm{~km} \times 10 \mathrm{~km}$ for user application. And the base maps of the UK are produced with these three resolutions respectively, as shown in Figure 2.5 where $1 \mathrm{~km} \times 1 \mathrm{~km}$ is chosen for demonstrating the example case for being consistent with the resolution of the dataset. 
In addition to setting the $\mathrm{OB}$, another important task at this step is to spatially index the data grids and label those that contain valid data. From now on, all subsequent spatial sampling is conducted over (or within, to be more precise) the base map.

\subsubsection{Sampling setup}

There are four initial settings (also seen in Figure 2.1b) that need to be specified before starting the sampling process which are:

1) Total numbers of samples required;

2) The desired location of the samples, which is only applicable in the case where users wish to fix the location while randomising other properties such as shapes and sizes;

3) Sample size in the unit of $\mathrm{km}^{2}$ which is translated into numbers of grids at the finest grid resolution used;

4) Spatial index of the ROI shape (i.e., samples) which is needed when a shapeconstrained sampling is required. In this case, the ROI shapes are randomly generated as convex hulls having the spatial index $(s p)$ value set by the user. In the case of shapeunconstrained sampling, the shape of the ROI's will be randomised. The spatial ( $s p)$ is defined to indicate dominant spatial extension direction, e.g., north-south or westeast:

$$
s p=\frac{\mathrm{D}_{\mathrm{NS}}}{\mathrm{D}_{\mathrm{WE}}}
$$

Where $\mathrm{D}_{\mathrm{NS}}$ and $\mathrm{D}_{\mathrm{WE}}$ refer to the north-south dimension (the longest vertical axis) and the eastwest dimension (the longest horizontal axis) of a sample (represented by a matrix). The reason for having $s p$ as an attached indicator is that in many climate studies, the direction of an area (such as a river catchment) plays a crucial role in determining the amount of quantity, such as rainfall (Svensson and Rakhecha, 1998; Viviroli et al., 2003). Obviously, other indexes, such as the direction of the major axis, can be easily defined if required. 


\subsubsection{Sampling processing and validation}

This is the final step (Figure 2.1c) where samples are generated according to the initial settings. The methods discussed below correspond to the three main functions of the SRS-GDA toolbox.

- Sampling with randomised locations

This function randomly selects different locations of centroids of the samples within the OB base map. The sampling is relatively straightforward: first $x$ - and $y$-coordinates are sampled from the range of the $\mathrm{OB}$ maps in the two directions using a joint uniform distribution $U(X, Y)$; followed by filtering out those samples that are not entirely within the OB.

- Sampling with randomised sizes

The second function is to randomly generate samples of different sizes, which is mainly used in the cases where the behaviour of aggregated quantity over the area of a sample is desired. Since the grid resolution $A_{\text {grid }}$ (in $\mathrm{km}^{2}$ ) is known, the size of the sample $A_{\text {sample }}$ can be translated into the number of grids $N_{\text {grid of sample }}$ of the ROI. The equation below shows the translation:

$$
N_{\text {grids of sample }}=A_{\text {sample }} / A_{\text {grid }}
$$

The variation of the area of the ROI (the sample) is realised by applying a 'buffer' operation while keeping the centroid location unchanged, i.e., it only increases or decreases the main axis of the sample proportionately. Figure 2.2 shows an example of shape generation. 


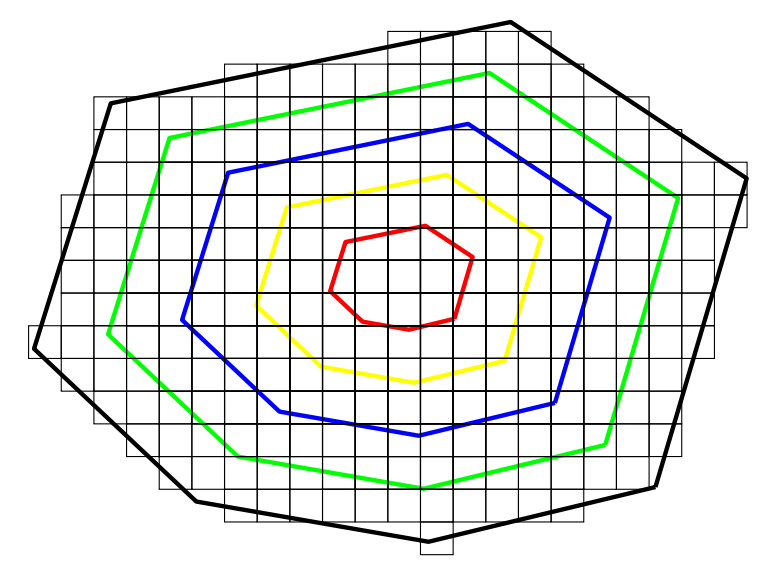

Figure 2.2 The 'buffering' operation used to vary the ROI into different sizes (shown here in different border colours).

- Sampling with randomized shape of ROI: unconstrained and constrained

The third main function is to randomly generate samples in different shapes varying in both sizes and locations. Depending on the user's initial settings, this function can conduct both shape-unconstrained and shape-constrained sampling. For the case of shape-unconstrained sampling, the location and the size of the sample (ROI) are both obtained from the two previous functions; for each combination of the location and the size, the shape is randomised using the size as a constraint. Two principles are applied in this process:

1) all grids should be interconnected, i.e., no isolated grids are allowed;

2) any growth must not go over the boundary set by the OB map.

The sampling starts at the given location and follows a random run to the neighbouring grid and records it until the number of grids equals the sample size. All the grids covered by the path are selected to comprise the sample. An extra validation step is applied to remove samples with the hole inside (the so-called ill-set samples) and rerun the process until the required number of samples is met, as presented in Figure 2.3. This sampling method can be used for generating the samples with free shape, especially for sampling from the area nearby the irregulated $\mathrm{OB}$. 


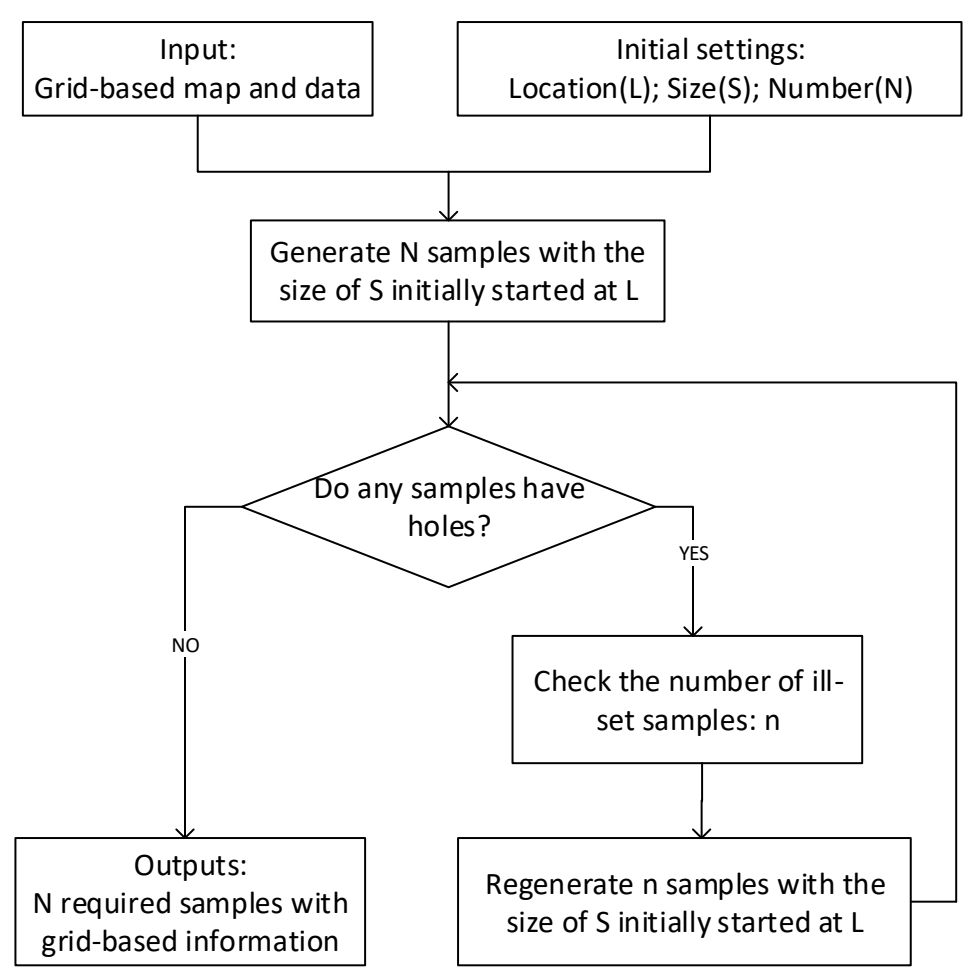

Figure 2.3 The process of shape-unconstrained random sampling method with ill-sample detection and removal.

For the case of shape-constrained random sampling, it focuses on sampling with the shapes of convex polygons as seen in many hydrological catchments in environmental or climatic research. The working flow is shown in Figure 2.4.

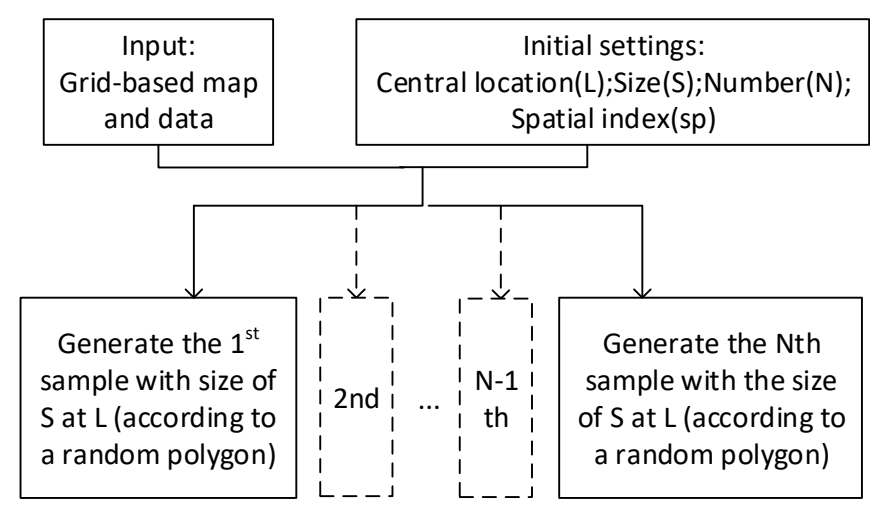

Figure 2.4 Flowchart of shape-constrained sampling method. 
Unlike the shape-unconstrained method, the shape-constrained random sampling method can control the shape of samples and produce more regular samples for mimicking river catchments or political areas. The main parameters such as the initial/centre location (L), sample size (S) and number $(\mathrm{N})$ are the same as those required by the shape-unconstrained method. In addition, the shape-constrained method uses one more major parameter "the spatial index $(s p)$ " as a further constraint. If required, several optional parameters can also be set to further refine the control of the polygon generation, i.e., the number of angles (usually is greater than or equal to 3 ) and the irregularity that indicates how much variance there is in the angular distance of vertices with a range of $0-1$; the spikiness which indicates how much variance there is in each vertex from the average radius with a range of $0-1$. However, as in the setup of the main parameters, L, S, N and $s p$, specification of these additional parameters is not compulsory. Unless otherwise specified explicitly by the user, default values of these additional parameters. The toolbox automatically generates default values for them will be applied (e.g., irregularity $=0.3$ and spikiness $=0.1$ ) to avoid generating extreme weird (irregularity $=1$ ) or sharp (spikiness $=1$ ) polygons. Compared with the shape-unconstrained random sampling method, it runs substantially faster because there is no need for random walking to grow the grids nor having any possibility of producing ill-set areas.

\subsection{An example application of the toolbox}

\subsubsection{Example dataset}

One of the motivations of this example is to investigate how areal rainfall extremes in terms of their distributions can vary with locations, size and shapes of the ROI. In fact, there has been a consensus about the impact of the size of catchment when producing areal rainfall at certain return levels. This is normally acknowledged by applying a so-called Areal Reduction Factor (ARF; Bell, 1976) to the value obtained at the location of the centroid of the catchment. Whilst variation of hydroclimatic variables is commonly recognised to be associated with climatology, the impact of the locations as well as the shape of the catchment have not been fully studied in 
a quantitative way. In this case, the GEAR dataset (see Table 1.1 on Page 20 of Chapter 1) is applied for this example.

\subsubsection{Application of the SRS-GDA toolbox}

To be consistent with the precision of the underlying dataset, the OB base map is produced as the same grid size of $1 \mathrm{~km}^{2}$. The production of the OB map undergoes two steps: first, a rough sketch of the boundary of Great Britain (GB) is used to generate grids with very coarse resolution set as $100 \mathrm{~km}^{2}$. This is to ensure that the boundary is properly covered. Secondly, the grid map is then refined by subdividing every grid with a number of smaller ones so that the grid resolution gradually increases to $5 \mathrm{~km} \times 5 \mathrm{~km}$ and $10 \mathrm{~km} \times 10 \mathrm{~km}$, which allows for the detection and removal of those grids falling outside of the boundary. This process is shown in Figure 2.5: (a) includes 638607 valid grids (marked as green) with the size of $1 \mathrm{~km}^{2}$; (b) includes 9464 valid grids with the size of $25 \mathrm{~km}^{2}$; (c) includes 2368 valid grids with the size of $100 \mathrm{~km}^{2}$.



(a)

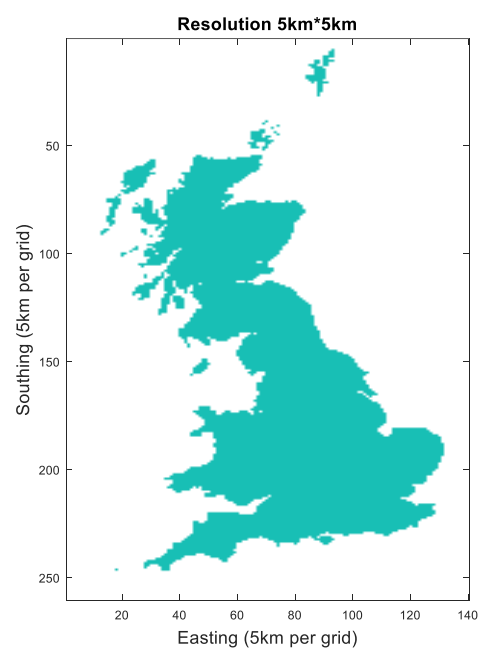

(b)

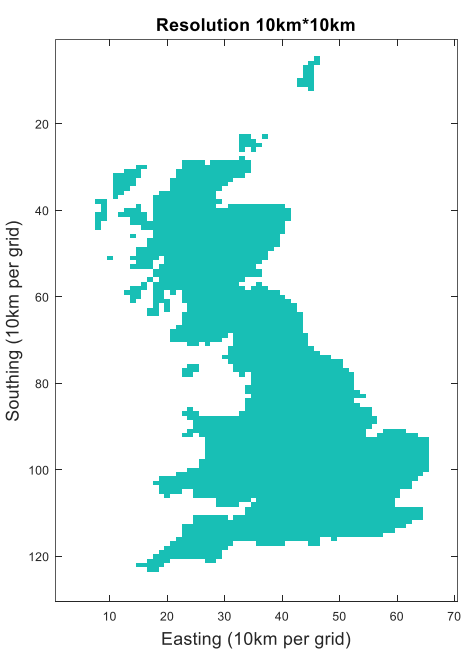

(c)

Figure 2.5 General map of Great Britain with three resolutions: (a) $1 \mathrm{~km} \times 1 \mathrm{~km}$ (b) $5 \mathrm{~km} \times 5 \mathrm{~km}$ (c) $10 \mathrm{~km} \times$ $10 \mathrm{~km}$. The difference in details and resolutions can be appreciated in the representation of the coastlines. 
Meanwhile, the initial centroid location of the sample in this example study is chosen to be at London with the coordinate of $\mathrm{L}=(520 \mathrm{~km}, 1070 \mathrm{~km})$. Two random sampling methods, e.g., shape-unconstrained and shape-constrained, are used to generate 5 different samples $(\mathrm{N}=5)$ at this location with the same size of $25 \mathrm{~km}^{2}$. According to Eq. (2.2), the number of grids in each sample (S) is calculated as $25 \mathrm{~km}^{2} / 1 \mathrm{~km}^{2}=25 . \mathrm{N}, \mathrm{L}$ and $\mathrm{S}$ are the basic inputs for the SRSGDA toolbox.

\subsubsection{Shape-unconstrained Random Sampling Method}

Table 2.1 presents the 5 different samples around the initial location L (grey grid) generated by the shape-unconstrained random sampling method. It can be observed that all samples have grids interconnected with no hole inside. However, the shapes of the sample can be very irregular as there is no requirement that they need to be a convex polygon which is used in the shape-constrained sampling method.

Table 2.1 Five example samples generated by shape-unconstrained sampling method.

\begin{tabular}{|c|c|c|c|c|c|}
\hline & No.1 & No.2 & No.3 & No.4 & No.5 \\
\hline Sample & ${ }_{\oplus}$ & $\forall+\|_{1}+\square$ & ${ }^{\#}$ & 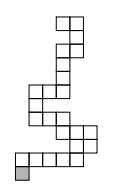 & 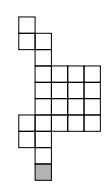 \\
\hline
\end{tabular}

The shape-unconstrained sampling offers maximum freedom; however, it can inevitably introduce shapes with holes inside, which have to be rejected.

Figure 2.6 shows the steps involved to detect and remove those ill-set sample shapes: First the original sample is presented to the validation function (Figure 2.6a) before it is converted into a binary image (Figure 2.6b). Secondly, the inner area of the binary image is flooded to remove the potential holes which results in a hole-free image as shown in Figure 2.6c. Finally, by comparing the areas of the two images, the location and the size of the hole(s) can be detected, which in turn triggers the removal process to discard the ill-set sample. In this test, the whole process of shape-unconstrained random sampling method takes 7.0 seconds on a low- 
configuration laptop to randomly generate five accepted samples with sizes of $25 \mathrm{~km}^{2}$ (specified as an initial constraint) while three samples are abandoned.
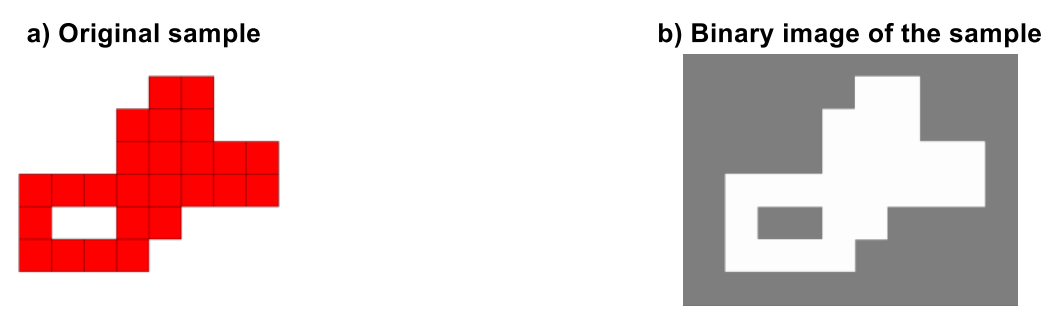

c) Filling holes

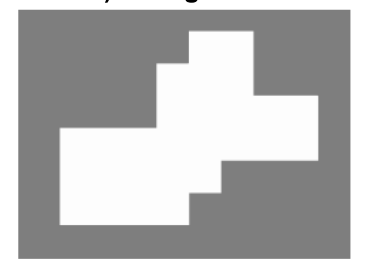

d) Holes detection

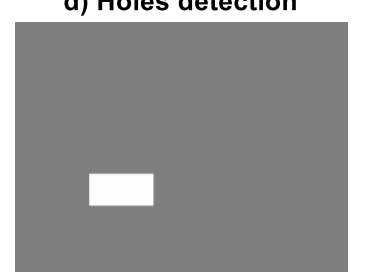

Figure 2.6 The process of hole detection.

\subsubsection{Shape-constrained Random Sampling Method}

Five samples at the same location L (grey grid) generated by using shape-constrained random sampling method are shown in Table 2.2 with various shape indexes $s p$ defined by the toolbox. Comparing with those samples listed in Table 2.1, clearly the shapes are more regular here as convex polygons, which can be directly used to simulate hydrological catchments. The whole process is recorded to have finished in 2.0 seconds on the test PC, which is faster than the former method. However, the tests show that the larger size and number are, the more efficient and time-saving the shape-constrained method is, compared with the shape-unconstrained method in Table 2.3.

Table 2.2 Five example samples generated by shape-constrained sampling method.

\begin{tabular}{|l|l|l|l|l|l|}
\hline & No.1 & No.2 & No.3 & No.4 & No.5 \\
\hline
\end{tabular}




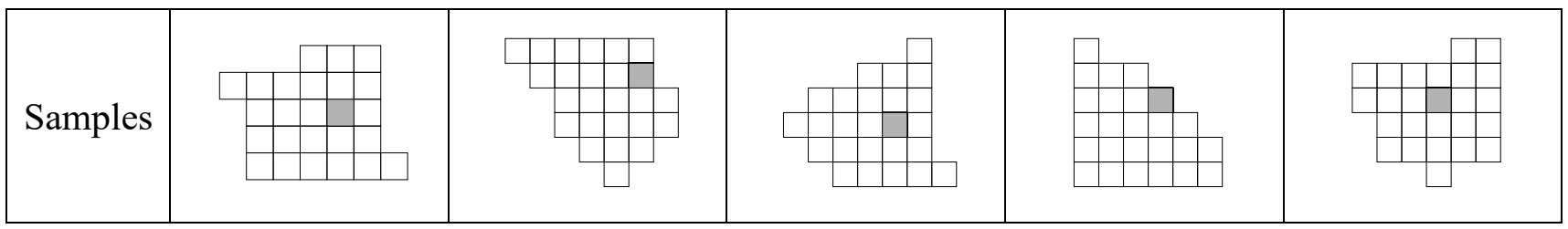

Table 2.3 Comparison of the indicative speed of the two sampling methods: Method 1 the shape-unconstrained method and Method 2 the shape-constrained method. Note that the numbers are obtained on the test PC and for comparing the relative speed difference.

\begin{tabular}{|c|c|c|c|c|c|c|c|c|}
\hline \multirow{2}{*}{$\begin{array}{l}\text { Number } \\
\text { of Grids }\end{array}$} & \multirow{2}{*}{$\begin{array}{c}\text { Sampling } \\
\text { Method }\end{array}$} & \multicolumn{7}{|c|}{ Number of Samples } \\
\hline & & 5 & 10 & 20 & 45 & 60 & 100 & 150 \\
\hline \multirow{2}{*}{25} & Method 1 & $7.4 \mathrm{~s}$ & $13.8 \mathrm{~s}$ & $39.1 \mathrm{~s}$ & $2.3 \mathrm{~min}$ & $3.6 \mathrm{~min}$ & $9.3 \mathrm{~min}$ & $20.9 \mathrm{~min}$ \\
\hline & Method 2 & $2.2 \mathrm{~s}$ & $2.8 \mathrm{~s}$ & $3.0 \mathrm{~s}$ & $4.6 \mathrm{~s}$ & $5.6 \mathrm{~s}$ & $7.0 \mathrm{~s}$ & $10.0 \mathrm{~s}$ \\
\hline \multirow{2}{*}{50} & Method 1 & $18.1 \mathrm{~s}$ & $33.1 \mathrm{~s}$ & $1.8 \mathrm{~min}$ & $8.1 \mathrm{~min}$ & $29.4 \mathrm{~min}$ & $39.6 \mathrm{~min}$ & $1.4 \mathrm{~h}$ \\
\hline & Method 2 & $2.1 \mathrm{~s}$ & $3.8 \mathrm{~s}$ & $4.7 \mathrm{~s}$ & $7.7 \mathrm{~s}$ & $10.7 \mathrm{~s}$ & $12.0 \mathrm{~s}$ & $20.6 \mathrm{~s}$ \\
\hline \multirow{2}{*}{100} & Method 1 & $50.8 \mathrm{~s}$ & $3.5 \mathrm{~min}$ & $28.1 \mathrm{~min}$ & $1.2 \mathrm{~h}$ & $2.4 \mathrm{~h}$ & $9.6 \mathrm{~h}$ & $12.9 \mathrm{~h}$ \\
\hline & Method 2 & $1.7 \mathrm{~s}$ & $3.1 \mathrm{~s}$ & $7.3 \mathrm{~s}$ & $11.4 \mathrm{~s}$ & $13.5 \mathrm{~s}$ & $23.0 \mathrm{~s}$ & $30.5 \mathrm{~s}$ \\
\hline
\end{tabular}

Figure 2.7 summarises the steps taken for shape-constrained sampling starting with an arbitrary but convex polygon (with $s p$, irregularity and spikiness all set by the toolbox) set at the same location index L (grey grid).

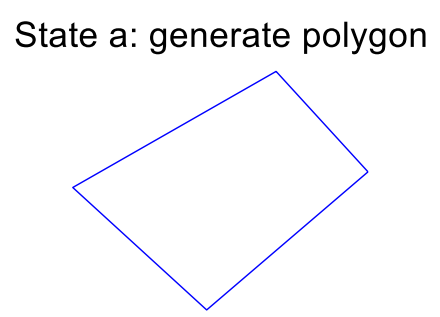

State b: select grid inside

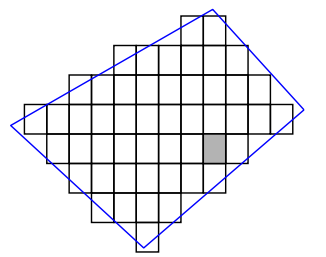

State c: final sample

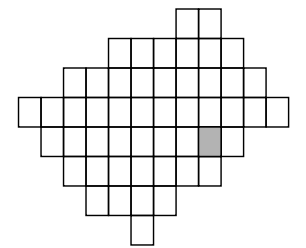

Figure 2.7 The process of generating samples by shape-constrained sampling method.

The effect of the spatial index $s p$ in the process of shape-constrained sampling is shown in Figure 2.8 with larger values of $s p$ having more north-south direction dominated shapes while 
smaller values indicate west-east direction dominated samples. Apparently, other shape-related constraints can be defined and applied subject to the needs of different applications.
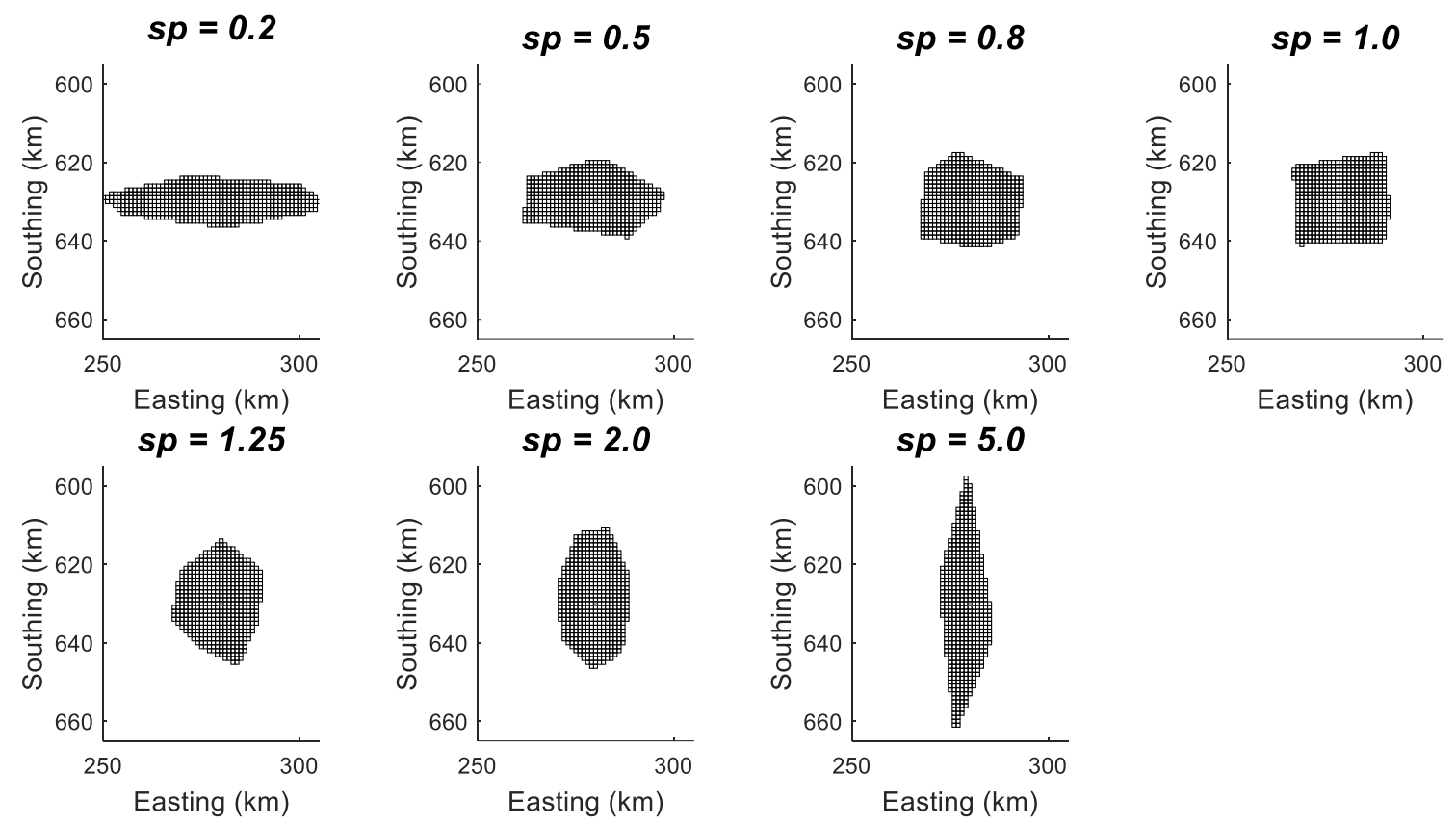

Figure 2.8 The seven samples with different spatial index $s p$.

The value of the toolbox can be well appreciated in the analysis results, partly shown in Figure 2.9 , in finding the spatial variation of extreme rainfall over GB. The entire analysis is not presented here; however, with the help of the SRS-GDA toolbox, I was able to reveal patterns never reported before. For example, a west-east variation of the rainfall distribution at different quantiles is clearly seen as "west high, east low" in Figure 2.9a. What is more interesting is the symmetric pattern shown in Figure $2.9 \mathrm{~b}$ (around $s p=1.0$ ) with regards to the sample shape which implies that sampled areas with slight elongation in north-south direction are expected to have a higher amount of rainfall than those spread more in the east-west direction at given frequency/return period. For samples with the same size and location, there is a remarkable difference of areal averaged rainfall between more elongated (e.g., $s p=0.2$ or 5.0) and rounded shape (e.g., $s p=1.0$ ) which can be attributed to heterogeneity of the grid rainfall distribution that cannot compensate to the areal average. The relationship between the sample size and the annual maximum daily rainfall (Figure 2.9c) is shown to have largely followed what is 
expected, e.g., decrease of areal rainfall as catchment size grows. These spatial features and underlying discussion are described in detail in Chapter 3.

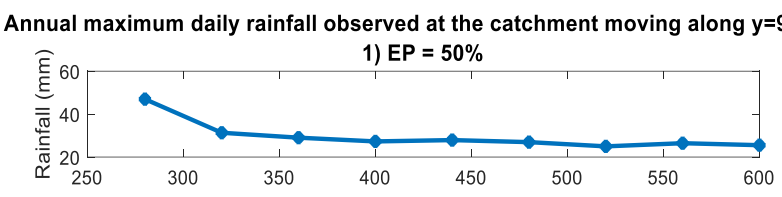

2) $E P=25 \%$
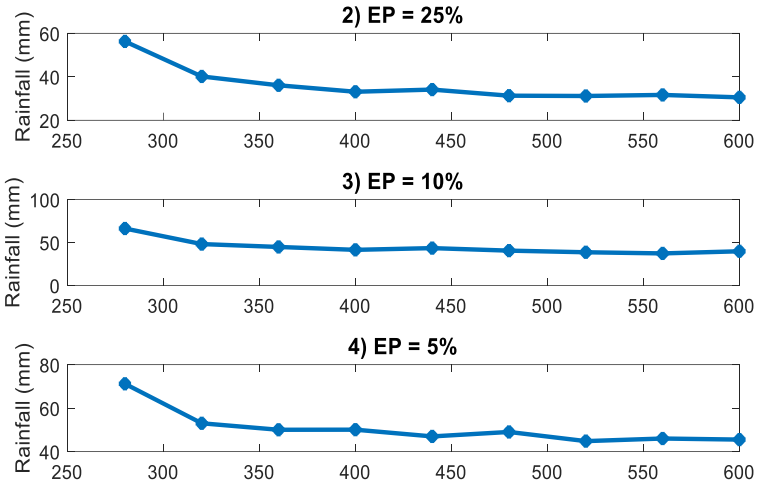

5) $\mathrm{EP}=1 \%$

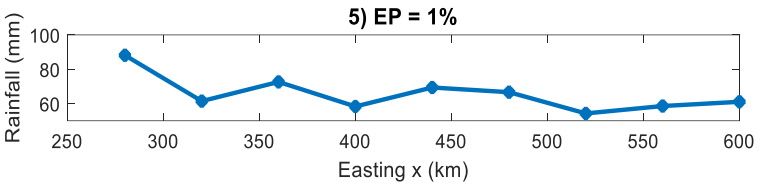

(a)
Annual maximum daily rainfall observed in the catchment central located at
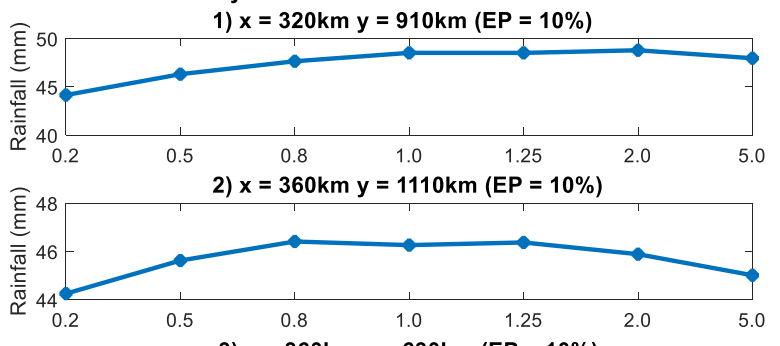

3) $x=360 \mathrm{~km} \mathrm{y}=630 \mathrm{~km}(E P=10 \%)$

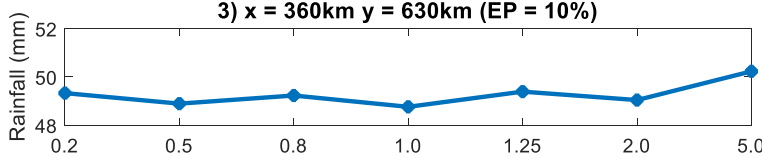

4) $x=400 \mathrm{~km} y=910 \mathrm{~km}(E P=10 \%)$
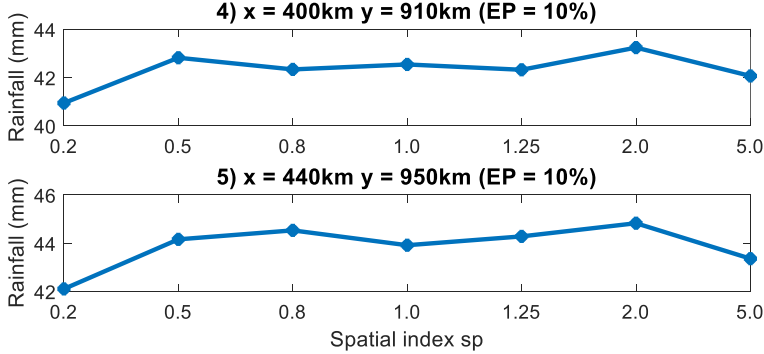

(b)

Annual maximum daily rainfall observed in the catchment central located at
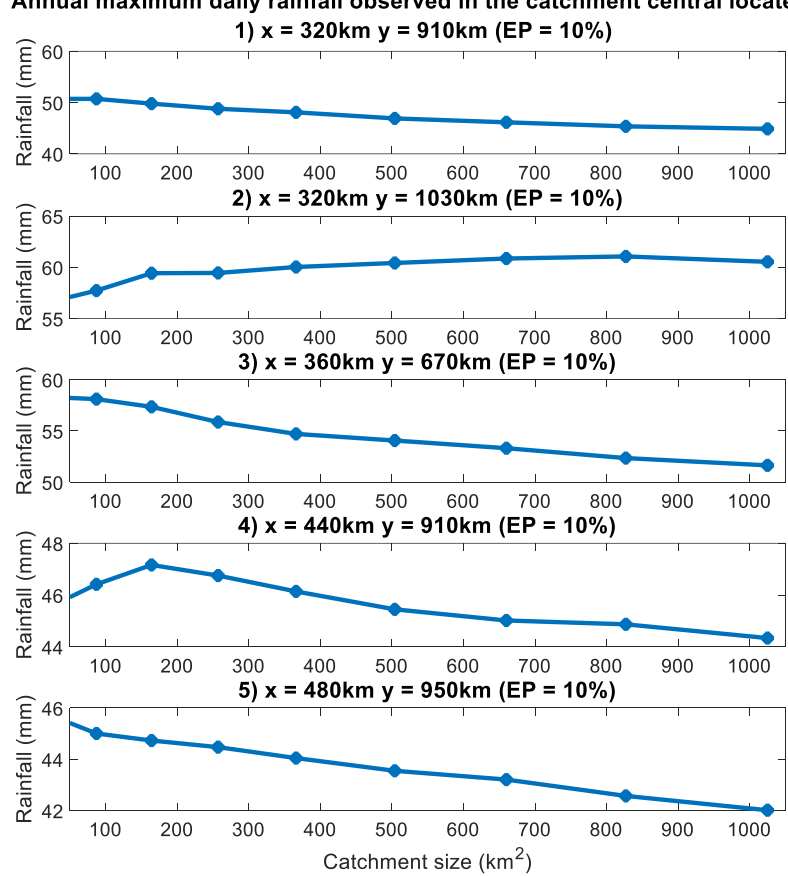

(c) 
Figure 2.9 The dependencies on the locations, the shape index and the size of rainfall distribution over GB: (a) the east-west pattern and (b) the symmetric pattern with regards to the sampled shape and (c) the trend pattern with regards to the sampled area size as detected by using the toolbox discussed in this chapter. EP is short for

"Exceedance Probability".

\subsection{Summary and remarks}

This chapter discusses the development of a new MATLAB toolbox for spatial random sampling in grid-based data analysis (SRS-GDA). The main aim of the toolbox is to address the very needs of many climate change related studies on spatial-temporal diagnostics of hydroclimatic datasets. An example application case is given in which the implementation details are discussed. The initial applications show that with this toolbox, several important variation patterns of extreme rainfall over GB that have yet to be reported are clearly identified. Based on the promising results and thanks to the availability of its source code, this toolbox will help the related research community in their analyses of grid data sets and gain further insight into the underlying science.

The source code of the SRS-GDA toolbox as well as the example case given in this chapter are also available at the GitHub (https://github.com/wanghan924/SRS-GDA Toolbox.git, published in doi: 10.5281/zenodo.4044626). The source code is provided subject to a GPL V3 licence. Use/fork of the toolbox is subject to proper acknowledgement as stated on the Webpage of the toolbox.

The following research on spatiotemporal variation of extremes (Chapter 3 and Chapter 4) is based on the SRS-GDA toolbox which is used to generate a large number of regions of interest (ROIs, i.e., so-called "sample" by the concept of toolbox in this chapter). 


\section{Chapter 3 Modelling Spatial Variation of Rainfall Extremes Using an ROI-based Approach}

With the support of the SRS-GDA toolbox depicted in Chapter 2, this chapter ${ }^{4}$ presents an ROI-based approach of modelling the spatial variation of rainfall extremes, aiming to address (part of) Q2 and Q3. The spatial variation of area-orientated annual maximum daily rainfall (AMDR) is modelled by well-fitted generalised extreme value (GEV) distributions, over the last century in Great Britain (GB) and Australia with respect to three spatial properties: geographic locations, sizes and shapes of the more than 11,000 regions of interest (ROI) that are generated by SRS-GDA toolbox and 903 real catchments and sub-catchments in the same river basin located in England and Wales that are used for comparison. The results show that the spatial variation of GEV location and scale parameters is dominated by geographic locations and area sizes. In GB, there is an eastward-decreasing banded pattern compared with a concentrically increasing pattern from the middle to coasts in Australia. The parameters tend to decrease with increased area sizes in both studied regions. Although the impact of the ROI shapes is insignificant, the round-shaped regions usually have higher-valued parameters than the elongated ones. The findings provide a new perspective to understanding the heterogeneity of extreme rainfall distribution over space driven by the complex interactions among climate, geographical features, and the practical sampling approaches.

\footnotetext{
${ }^{4}$ Part of the contents of Chapter 3 has been published in "Wang, H., \& Xuan, Y. (2020). Spatial variation of extreme rainfall observed from two century-long datasets. Geophysical Research Letters, 48, e2020GL091933. https://doi.org/10.1029/2020GL091933." And part of the contents has been revised and resubmitted to the journal "Atmospheric Research".
} 


\subsection{Introduction}

Intensive rainfall is considered to be one of the primary triggers for flooding alongside other factors such as climate change, topography, and soil type of different catchment patterns (Rogger et al., 2017; Westra et al., 2014). In this chapter, a modelling framework is proposed to gain insights into how area-orientated rainfall extremes vary with space with respect to the probability distribution parameters which are of concern of flood risk management and civil engineering design. By making use of two century-long, grid-based rainfall datasets (the GEAR dataset and the ADAM dataset, see Table 1.1 on Page 20 of Chapter 1) covering Great Britain (GB) and Australia (AU), the study attempts to address the research questions (part of) Q2 and Q3, where the following sub-questions are specified as:

1. How areal rainfall extremes change over space (or elevation).

2. How other factors such as the size, shapes (orientations) of the area in question may affect such spatial dependencies.

3. How the spatial patterns and variations are linked to the large-scale climatology of rainfall.

4. What is the implication of the spatial variation of the parameters to the applications (e.g. flood risk management).

In addition, the SRS-GDA toolbox described in Chapter 2 is employed to assist the required spatial sampling and generate 11,011 ROIs with predefined spatial features, i.e., geographic location, size and shape. In parallel with these arbitrary ROIs, 903 real catchments in England and Wales are also employed to help validate the result of the ROI-based study. The spatial features of catchments are identified and extracted by the Spatial Pattern Extraction and Recognition (SPER) toolbox described in Chapter 7. The sampled annual maximum daily rainfall (AMDR) at each ROI or catchment is fitted with the widely used and tested Generalised Extreme Value (GEV) distributions whose spatial variation is then analysed. The associated intensive computation demand is met by the high-performance computing (HPC) resources provided by Super Computing Wales (https://www.supercomputing.wales). 
The remainder of this chapter is organised as follows: Section 3.2 describes the methodology of spatial variation analysis including generation of ROIs, spatial feature extraction of catchments, fitting GEV and goodness of fit (GOF) tests. Then GOF test result is presented in Section 3.3. And both the qualitative and quantitative results of the spatial variation of the distribution parameters in ROIs are discussed in Section 3.4. Meanwhile, the analysis of the spatial distribution of parameters in catchments is discussed in Section 3.5. The linkage between the spatial distribution of GEV parameters with the large-scale climatology of rainfall is explained in Section 3.6. Finally, the summary of the conclusion and recommendations of further study are given in Section 3.7.

\subsection{Methodology}

The methodology considers two different subjects when it comes to the area of interest as described in Section 3.1: 1) that is defined by the ROIs generated by the SRS-GDA toolbox and 2) that is just real catchments. While areas based on variable and arbitrary ROI are used to explore the relationship among extreme rainfall and spatial characteristics such as location, size and shape which can vary with different study purposes, the study of real catchments is used to validate such relationships. Therefore, the first step of the methodology is to generate ROIs and identify the spatial features of all ROIs as well as those of the real catchments. In one aspect, the geographical areas of the two data domains, i.e., GB and AU, are firstly sampled into a series of ROIs using the SRS-GDA toolbox and three different types of predefined spatial features (geographical locations, sizes and shapes) are applied in this spatial sampling process to reduce the overall computing time while maintaining the representativeness of the samples. As a result, these ROIs are evenly distributed across the two study domains. In the other aspect, since the boundary of each real catchment in England and Wales has already known, the SPER toolbox is employed to extract the spatial features (geographical locations, elevation, size, orientation and shape), e.g., no variations of the spatial features as those done with ROIs. Then the AMDR extracted from each ROI and catchment is fitted with a probability distribution. In this study, the three-parameter GEV distribution is chosen as the candidate distribution. 
Meanwhile, the goodness of fit (GOF) of the fitted distributions is further tested by two different methods: the Kolmogorov-Smirnov (KS) and Anderson-Darling (AD) tests. The location and scale parameters ( $\mu$ and $\sigma$ ) of the fitted distributions are then analysed with regard to their spatial distribution with reference to the large-scale climatology of rainfall variations.

\subsubsection{ROI generation, catchment identification and AMDR extraction}

The ROI sampling starts with an initial set of uniformly distributed ROIs whose geographical locations are recorded as the coordinates of the geometric centroid of ROIs. The 7 shapes of these ROIs are predefined and parameterised by their spatial indexes (see Eq. (2.1) on Page 35 ) reciprocally grouped as $0.2 / 5.0,0.5 / 2.0,0.8 / 1.25$ and 1.0 . The size of these ROIs is then gradually increased by 10 steps with a $20 \%$ increment each, while maintaining the same shape and location (of the geometric centroid). In the end, the largest sizes of the ROIs are $1,050 \mathrm{~km}^{2}$ for $\mathrm{GB}$ and $9,900 \mathrm{~km}^{2}$ for AU respectively.

The SRS-GDA toolbox used to generate the ROIs is set up in a way that only one spatial feature is allowed to vary at a time. For instance, to obtain ROI samples of G2 and A2 in Table 3.1, the toolbox is configured to keep the centroid location unchanged while generating 10 ROIs only by varying their sizes. Table 3.1 also summarises all ROIs and their properties.

Table 3.1 ROIs for analysing the spatial variations in GB and AU.

\begin{tabular}{|c|c|c|c|c|}
\hline \multicolumn{2}{|c|}{ Sampling areas } & Changing with location & $\begin{array}{l}\text { Changing with size } \\
\text { (each group includes } 10\end{array}$ & $\begin{array}{l}\text { Changing with shape } \\
\text { (each group includes } 7\end{array}$ \\
\hline \multirow{3}{*}{$\begin{array}{l}\mathrm{G} \\
\mathrm{B}\end{array}$} & Indicator & G1 & G2 & G3 \\
\hline & $\begin{array}{l}\text { ROI(s) of } \\
1 \times 1 \mathrm{~km} \\
\text { grid }\end{array}$ & 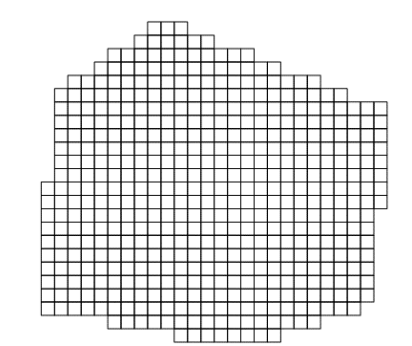 & 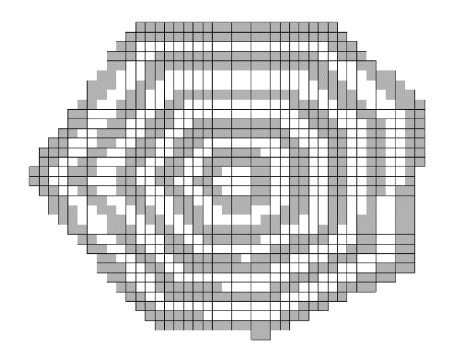 & $\begin{array}{c}\mathrm{sp}=0.2 \\
\mathrm{sp}=0.5\end{array}$ \\
\hline & Size $\left(\mathrm{km}^{2}\right)$ & 500 & $\begin{array}{c}10,43,87,164,257,366 \\
504,660,827,1025\end{array}$ & 500 each ROI \\
\hline
\end{tabular}




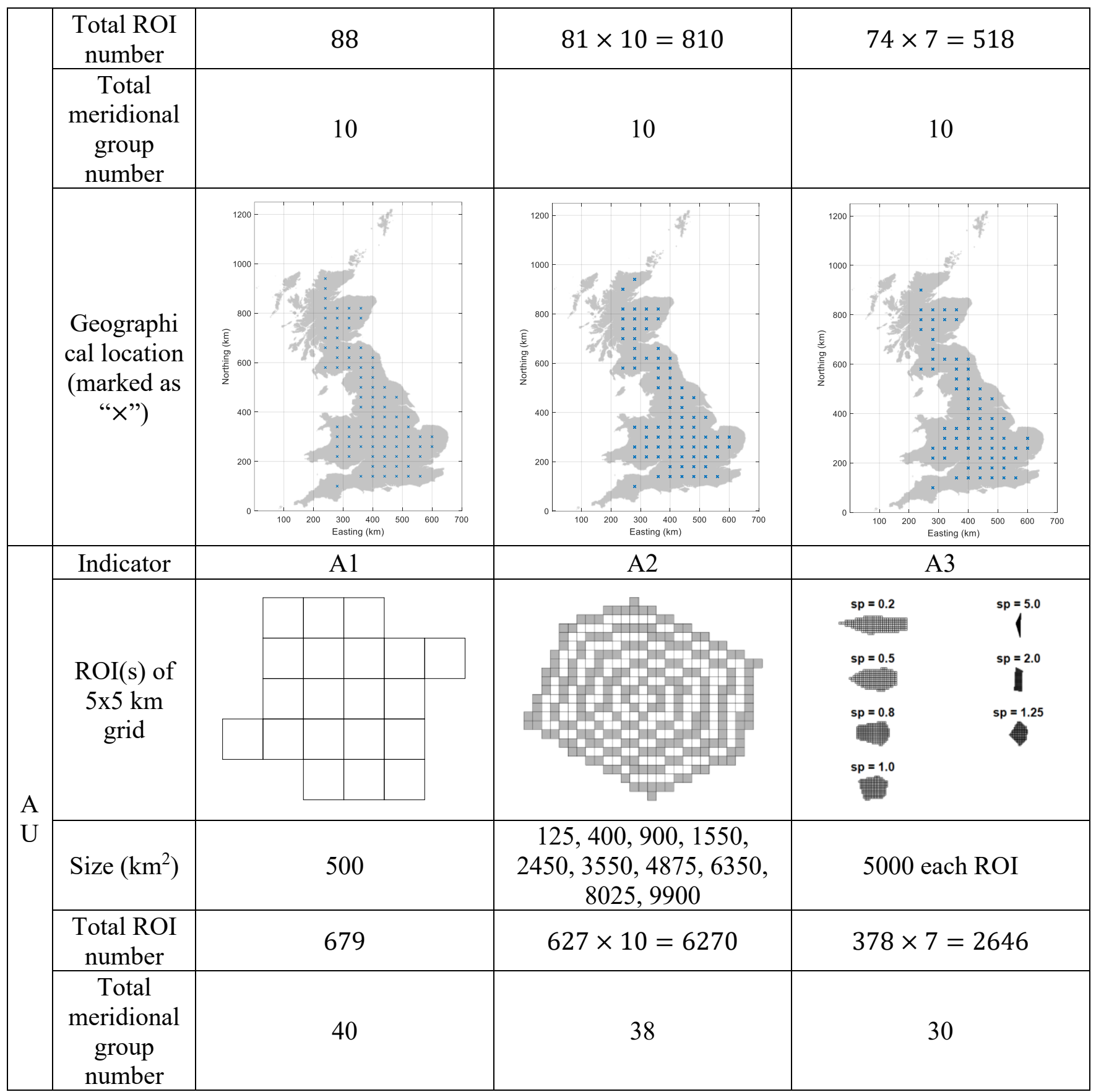




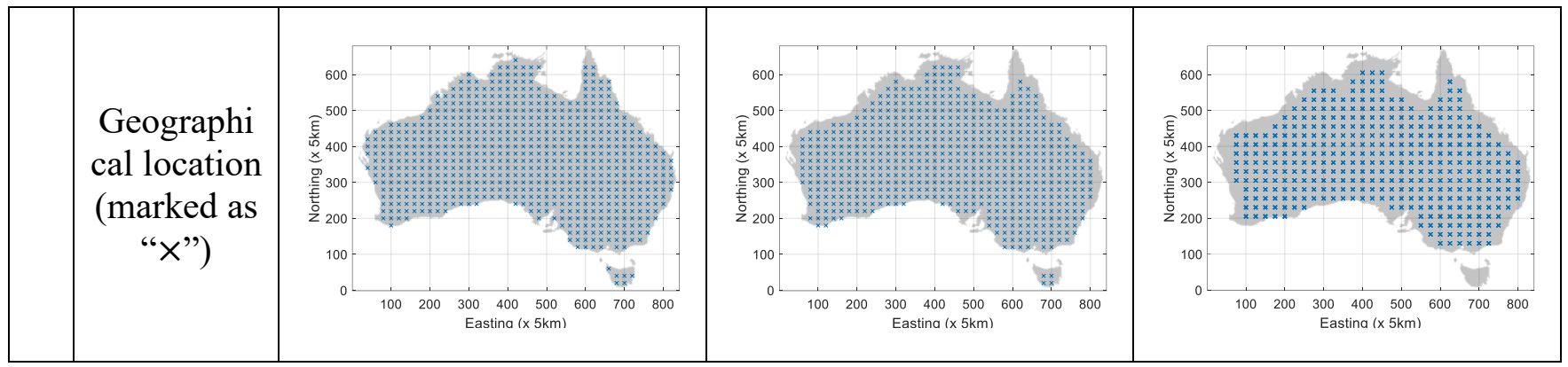

As for the analysis of rainfall extremes over real catchments, 903 catchments of England and Wales are included and their locations are presented in Figure 3.1a.

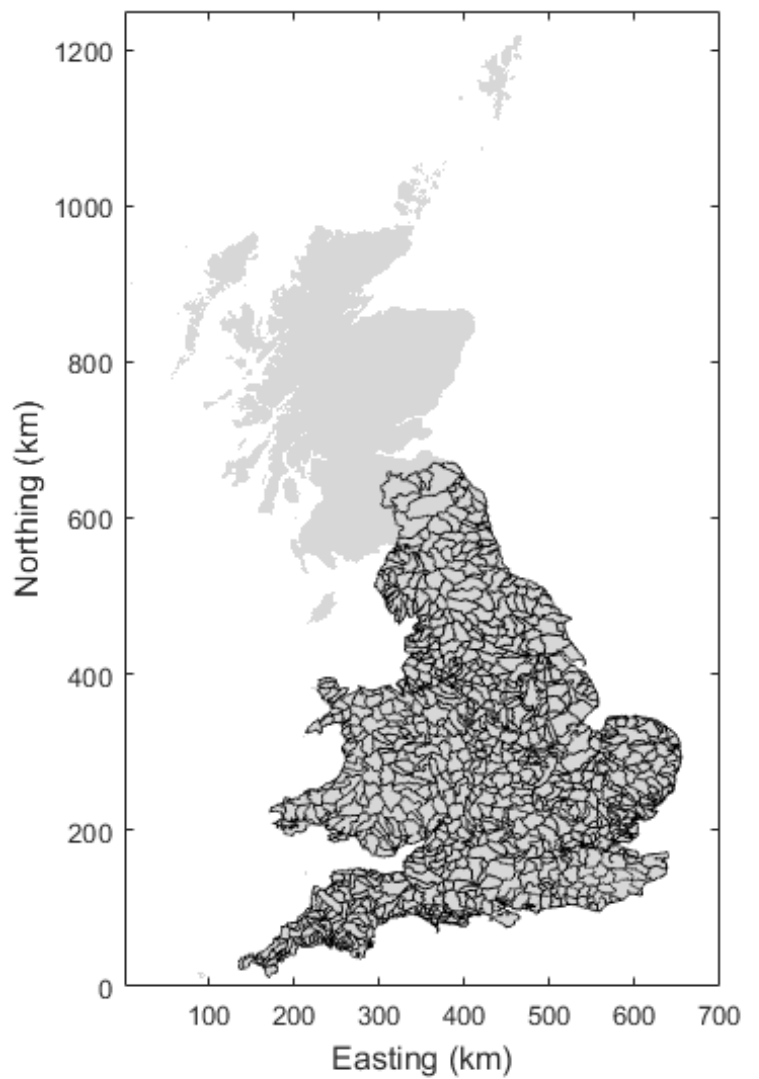

(a)

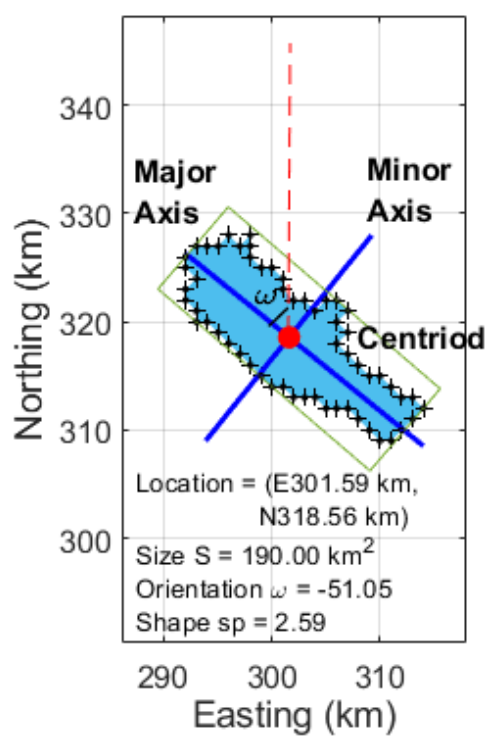

(b)

Figure 3.1 Location of the catchments over England and Wales (a) and one catchment with its spatial features is exemplified (b).

Different from those of the ROIs, the location, boundary and shape of the real catchments have been already given. In order to validate the ROI-based study, the spatial indexes used for representing the spatial characteristics of studying regions should be consistent to make two 
studies comparable. Therefore, the SPER toolbox (see Chapter 7) is employed to identify and quantify the spatial features of these catchments such as the location, size, and shape/orientation in a similar way. The location of the catchment is represented by the coordinates of its geometric centroids under the system reference system (national grid reference, NGR) of the GEAR dataset while the size is calculated as the number of grids inside the boundary (as the grid size is $1 \times 1 \mathrm{~km}^{2}$ ). The only slight difference is in defining the shape and orientation of the catchments. In the ROI-based study, the shape index $s p$ is defined as the ratio of north-south dimension over east-west dimension because the shape of ROI generated in the study (see A3 and G3 in Table 3.1) is either north-south orientated or east-west orientated. However, such a definition is not broad enough in describing the case of real catchments which have various orientations. Thus in the catchment-based study, the SPER toolbox is used to find the main axis of the catchment and the orientation $\omega$ is defined as the angle of major axis from the North in clockwise direction (One example catchment can be seen in Figure 3.1b).

Finally, for each catchment there are four indexes used to represent the spatial features that are used to compare with ROIs, including 1$)$ the location index ( $x$ - and $y$ - of the geometric centroid); 2) the elevation of the catchment; 3) the size index $s$ which is the size of the catchment; 4) orientation index includes the ratio of major axis and minor axis (which is perpendicular to the main axis), $s p$, and the angle $\omega$. Besides, the average elevation of all catchments is calculated by using the OS Terrain 5 dataset (available in https://www.ordnancesurvey.co.uk/business-government/products/terrain-5). This dataset is supplied as a whole set of GB divided into $5 \mathrm{~km}$ by $5 \mathrm{~km}$ tiles. These tiles are identified by quoting the National Grid reference of the southwest corner of the area they cover and the dataset is published as both grid (with 5-metre post spacing) and contours (with 5-metre interval). In this study, a grid type is set such that each tile includes 100 by 100 (10000) grids whose size is $50 \times 50 \mathrm{~m}^{2}$. In this study, to make the spatial resolution of two datasets consistent, a conversion of the resolution of the OS Terrain 5 data is done by firstly resampling the OS Terrain 5 data in the $50 \times 50 \mathrm{~m}^{2}$ grids, and then taking the average of height to match the $1 \times 1 \mathrm{~km}^{2}$ grids of the GEAR dataset. 
For each ROI and catchment, the areal daily rainfall is calculated by taking the arithmetic average and the maximum value of each year is selected to generate the annual maxima series, i.e., annual maximum daily rainfall, (AMDR). There is a huge amount of data to be processed, e.g., around 700 gigabytes of grid-based daily rainfall data $(1 \times 1 \mathrm{~km}, 365$ days/year, 113 years) covering the UK in our case, which causes a heavy overhead of typical desktop PC that usually features 4 processing cores and a limited memory capacity. To increase the operation efficiency, we employed supercomputers from the High-performance Computing (HPC) Wales (https://www.supercomputing.wales) to assist the extraction of such huge data. The supercomputing hubs of HPC Wales have a total of 13,080 processing cores, connecting to high-speed memory and storage, and can deliver 1 petaflop of computing power (Flanagan et al., 2020). Supercomputers of HPC Wales are Linux-powered and use the Slurm batch scheduler/script to allocate and push the jobs or programme to supercomputers and run (more operation details can be checked in https://portal.supercomputing.wales/). In this study, the programme for extracting catchment rainfall is coded in Python and multiprocess is used for paralleling the jobs to increase the computation speed.

\subsubsection{Fitting the extracted AMDRs using GEV distribution}

Derived from the extreme value theory, the generalised extreme value (GEV) distribution has become by far one of the most well-founded distributions for describing annual maximum rainfall. It has been applied to not only many gauged rainfall extreme studies (Feng et al., 2007; Martins and Stedinger, 2000; Westra et al., 2013) but also those using grid rainfall datasets (Overeem et al., 2010). For a given ROI or catchment, areal daily rainfall is calculated by taking the arithmetic average before the annual maxima series (Annual maximum daily rainfall, AMDR) is generated (denoted here as $x$ ). Then the series $x$ can be fitted by using the GEV whose cumulative distribution function is defined as:

$$
F(x ; \sigma, \mu, \xi)=\exp \left[-\left(1+\xi\left(\frac{x-\mu}{\sigma}\right)\right)^{-1 / \xi}\right]
$$

The cumulative probability function $F$ is defined for $1+\xi(x-\mu) / \sigma>0,-\infty<\mu<$ $\infty, \sigma>0$ and $-\infty<\xi<\infty$, where $\mu$ is the location parameter, $\sigma$ is the scale parameter, and 
$\xi$ is the shape parameter. There are three types of distribution in the GEV family which are distinguished by their shape parameters. Type I, also known as the Gumbel distribution, refers to the case where $\xi=0$; while the types II and III are known as the Fréchet distribution and the Weibull distribution corresponding to the cases where $\xi>0$ and $\xi<0$ respectively. A maximum likelihood estimator (MLE, Hosting, 1985) is introduced to estimate the three parameters of the GEV distribution fitted to those AMDRs extracted from each ROI.

It is worth revisiting the implication of parameters of the GEV models. The location parameter $\mu$ indicates the mode of the time series which is consistent with the most frequent AMDR in our cases, while the scale parameter $\sigma$ indicates its average dispersion for each AMDR from $\mu$ (equals $\sqrt{6} / \pi$ multiplying by the standard deviation if $\xi=0$ ) (Izaguirre et al., 2010). In other words, the larger $\sigma$, the more spread-out the distribution is. Conversely, the smaller the parameter, the more compressed the distribution is (Kantar and Şenoğlu, 2008; Mann, 1967). In our study, if $\sigma$ is estimated to be increasing, the occurrence probability of extreme AMDR, i.e. rainfall ranked in the higher positions is increased.

\subsubsection{Goodness of fit tests}

Although the GEV distribution generally fits well to the point rainfall extremes (e.g. gauge observation) as reported in many studies before (Schaefer, 1990; Yoon et al., 2013), very few have been done on the suitability of GEV distribution fitting the areal grid-based rainfall extremes. Therefore in this study, the goodness of fit by GEV distribution is tested using two methods: The Kolmogorov-Smirnov (KS) test (Kolmogorov, 1933; Smirnov, 1948) and Anderson-Darling (AD) test (Anderson and Darling, 1952, 1954). These two tests have been widely used to test whether a given data sample is drawn from a particular type of probability distribution (the reference distribution). In this case, the reference distribution is GEV distribution and the null hypothesis $H_{0}$ for both tests is that the $\operatorname{AMDR} X\left(x_{1}, x_{2}, \ldots, x_{n}\right)$ is drawn from GEV distribution and the alternative hypothesis $H_{1}$ states against $H_{0}$. Besides, the L-moment ratio diagrams are also employed to compare the fitted GEV distribution with the statistical characteristics of AMDR itself. 
- KS test

The KS test detects the greatest vertical distance, the so-called KS test statistic $\left(D_{n}\right)$, between empirical cumulative distribution function of the observed $\operatorname{AMDR} F_{n}(x)$ and the cumulative distribution function of the reference GEV distribution $F(x)$. The equation for test statistics is given by:

$$
D_{n}=\sup _{x}\left|F_{n}(x)-F(x)\right|
$$

where $\sup _{x}$ is the least upper bound of the set of distances. $F_{n}(x)$ can be calculated by $\frac{1}{n} \sum_{i=1}^{n} I_{X_{i} \leq x}$ where $I_{X_{i} \leq x}$ is an indicator function and equals 1 if $X_{i} \leq x$ or 0 if otherwise. If $H_{0}$ holds, $D_{n}$ tend to be small. Conversely, large values of $D_{n}$ are expected. The criteria are to reject the null hypothesis at a 0.05 significance level if $D_{n}$ is greater than the critical value (0.198).

- $\mathrm{AD}$ test

Similar to the KS test, the $\mathrm{AD}$ statistic $\left(A^{2}\right)$ is used in the $\mathrm{AD}$ test to detect how well the data follow a reference distribution, i.e., GEV distribution in this study. The smaller $A^{2}$ indicates a better fitness of the data by given distribution. Different from the KS test, the AD test weights more heavily in the tails of the distribution for extreme data and $A^{2}$ is given as:

$$
A^{2}=-n-\frac{1}{n} \sum_{i=1}^{n}(2 i-1)\left\{\ln F\left(X_{i}\right)+\ln \left[1-F\left(X_{n-i+1}\right)\right]\right\}
$$

If $A^{2}$ is greater than the critical value (2.502) at the 0.05 significance level, the null hypothesis is rejected. The critical value is approximated depending on the sample size only and not on the distribution.

One of the most significant limitations when using the non-parametric KS test in evaluating the fitness is that the reference distribution (i.e., the reference GEV distribution $F(x)$ in Eq. (3.3)) has to be fully specified and data-independent (Fasano and Franceschini, 1987). In other words, the KS test becomes invalid if the three GEV parameters are estimated using the same 
data whose distribution is going to be tested. Therefore, the challenge is how to determine the reference GEV distribution appropriately. One possible approach is to use the bootstrapping technique to simulate the reference. I broadly followed Lilliefors (1967) and developed a bootstrapping method to establish the reference distribution under the null hypothesis $H_{0}$ and the general procedure can be found in Eduardo (2020).

Step 1: Estimate a set of parameters $\hat{\theta}$ of the GEV distribution, from the sample of AMDR $\left(x_{1}, x_{2}, \ldots, x_{n}\right)$ by using the maximum likelihood (ML) method;

Step 2: Compute the KS statistic $D_{n}$ from $\boldsymbol{X}$ and the cumulative distribution function (CDF) of the GEV distribution with the parameters of $\widehat{\theta}$, e.g., $F_{\widehat{\theta}}$;

Step 3: Perform bootstrap resampling for a predefined number of times $J$; for each iteration $j=$ $1,2, \ldots, J:$

i. $\quad$ simulate a bootstrapped sample $\left(x_{1 j}, x_{2 j}, \ldots, x_{n j}\right)$ from $F_{\widehat{\theta}}$;

ii. estimate a new set of parameters $\hat{\theta}_{j}$ from the bootstrapped sample $\left(x_{1 j}, x_{2 j}, \ldots, x_{n j}\right)$ using the same ML method;

iii. compute the statistic $D_{n j}$ from $\left(x_{1 j}, x_{2 j}, \ldots, x_{n j}\right)$ and $F_{\widehat{\theta}_{j}}$;

Step 4: Obtain the $p$-value approximation:

$$
p \text {-value } \approx \frac{1}{J} \sum_{j=1}^{J} 1_{D_{n j}>D_{n}}
$$

In this study, $J=5000$ is used to run the iterations for each ROI. A significant level $\alpha=0.05$ is used and the null hypothesis $H_{0}$ is rejected if $p$-value $<\alpha$.

- The L-moment ratio diagrams

The L-moments method proposed by Hosking (1990) has been widely applied to select the most appropriate theoretical distribution to fit a given data sample by comparing the linear combinations of probability weighted moments, i.e., L-moments. The advantages of applying L-moment are that they can characterize a wider range of theoretical distributions and can be 
less affected by the presence of the outliers in samples and the bias in the estimation. The Lmoment method uses ratios to characterize distributions which are introduced below:

$$
\begin{gathered}
\mathrm{L}-\mathrm{CV}=\lambda_{2} / \lambda_{1} \\
\text { L-skewness }=\lambda_{3} / \lambda_{2} \\
\text { L-kurtosis }=\lambda_{4} / \lambda_{2}
\end{gathered}
$$

where $\lambda_{r}$ indicates the $r$-th L-moment. Let $X_{r-k: r}$ denote the $k$-th order statistic in an independent sample (i.e., AMDR time series) of size $n$ if the expectation $E X_{r-k: r}$ exists, $\lambda_{r}$ can be calculated as:

$$
\lambda_{r}=r^{-1} \sum_{k=0}^{r-1}(-1)^{k}\left(\begin{array}{c}
r-1 \\
k
\end{array}\right) E X_{r-k: r}
$$

\subsubsection{Analysing the spatial distribution of the location-scale parameters}

The spatial variation of the location and scale parameters of the fitted GEV distributions are analysed both qualitatively and quantitatively. Instead of using full spatial coordinates to represent the geographical locations, a univariate spatial-location representation is adopted in the ROI-based study. The procedure is briefly described below:

i. The chosen GEV parameter is aggregated meridionally, e.g., over all ROIs that have the same $x$-direction (easting or longitude) coordinate.

ii. The aggregated GEV parameter values are indexed by their $x$-direction only coordinate which is then used as an input variable to represent the geographical locations.

iii. The same procedure is also applied zonally, i.e., over the same $y$-direction coordinate.

With this arrangement, the meridional or zonal average of the GEV parameter in question is taken as the response variable (predictor). In AU, a concentric pattern is found where both the meridional average and the zonal average show a similar result while for the case of GB, only a strong west-east pattern exists (shown in Figure 3.2). Therefore, for comparing two cases and convenience, the meridional average is taken for both cases. 

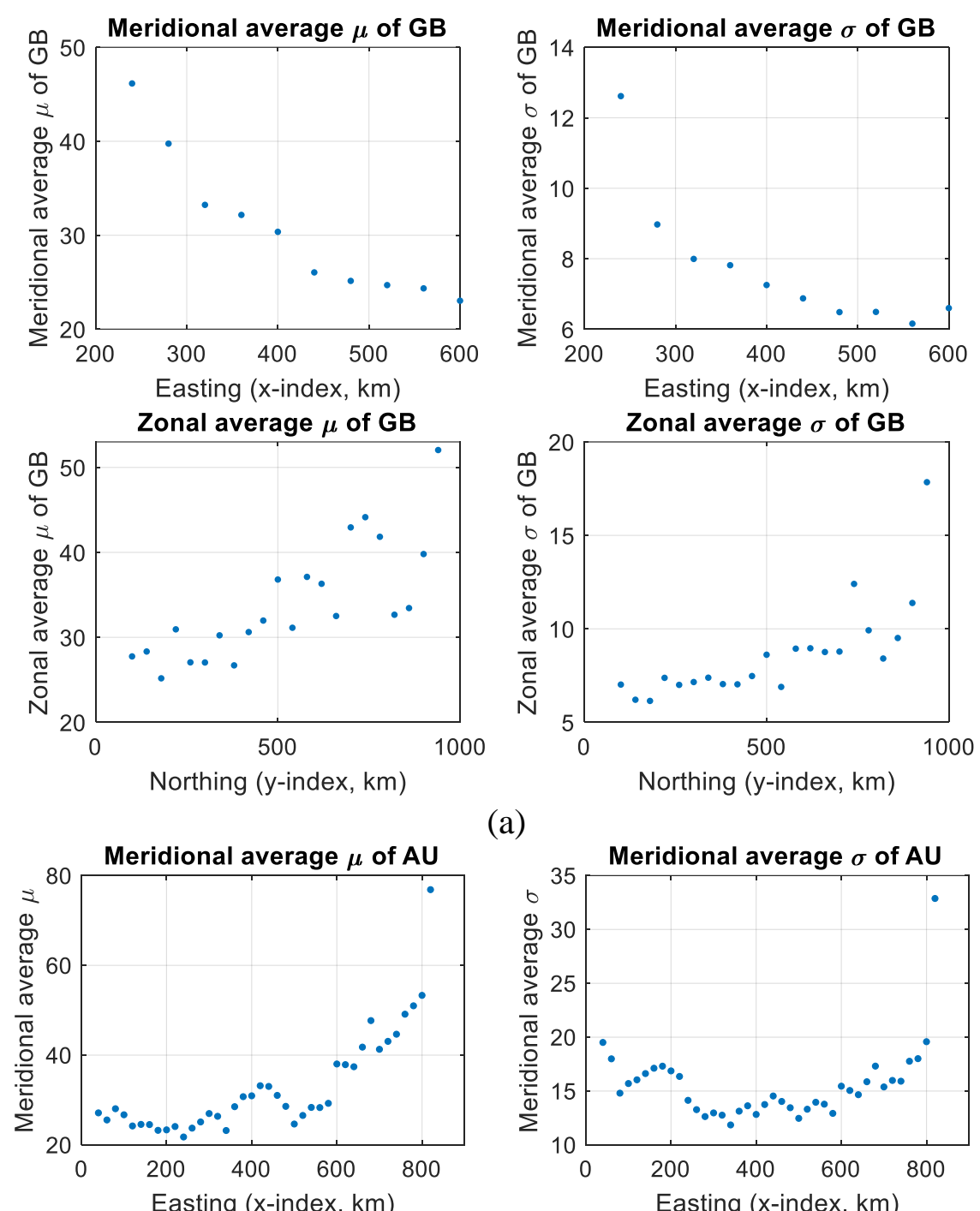

(a)
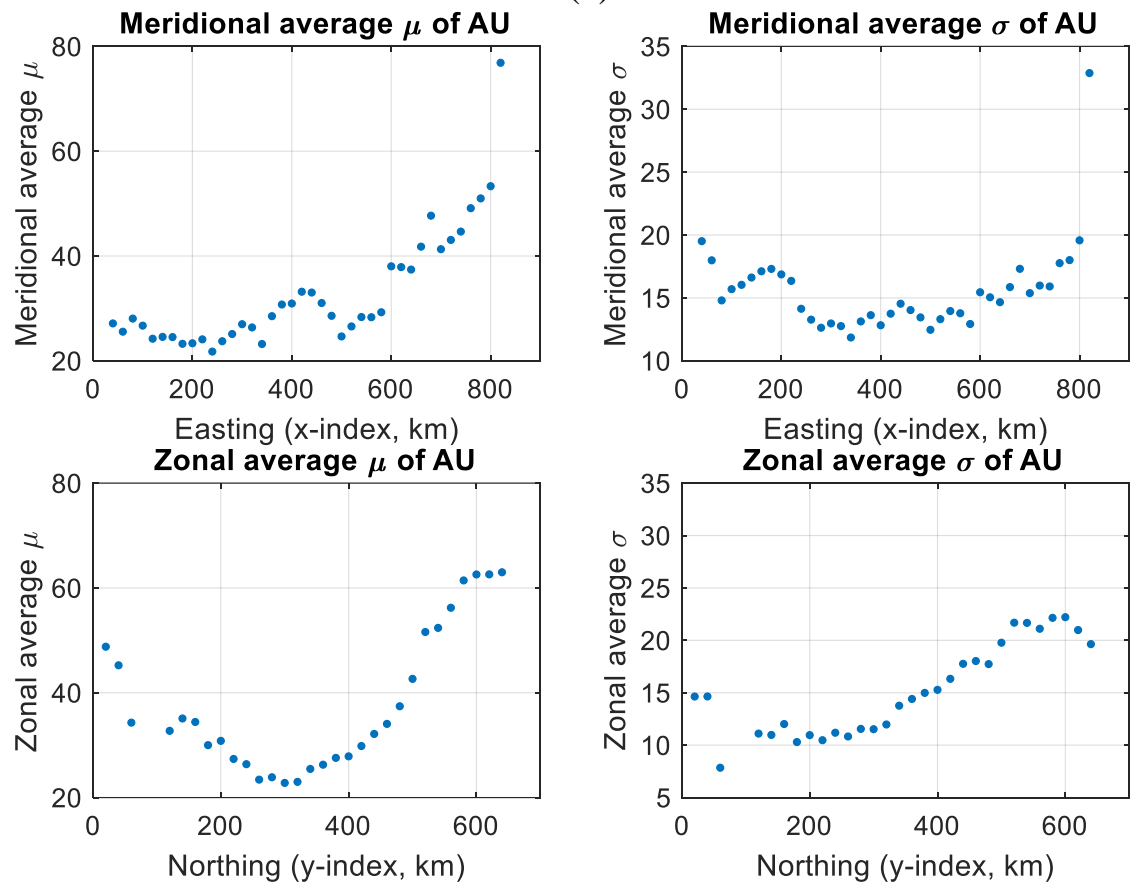

(b)

Figure 3.2 Zonal and meridional average parameter analysis in Great Britain (GB) and Australia (AU).

Finally, a generalised linear model (GLM) is fitted to quantify the relationship between the GEV parameters and the associated spatial features, i.e., to explicitly model the spatial variation 
of the GEV parameters with respect to the locations, sizes, and shapes of the underlying ROIs. The setups of the structure of GLM are based on qualitative analysis of location and scale parameters, which is shown in Section 3.4.4 in detail. A $k$-fold-cross-validation (Efron and Tibshirani, 1997) and variogram (Cressie and Hawkins, 1980) are introduced to evaluate the performance of the GLMs.

\subsection{Results of goodness of fit tests}

The following Figure 3.3 shows the histogram of test statistics of exampled 88 ROIs in GB by using a bootstrapping method and the title of each histogram indicates the $p$-value and the location of ROI in bracket. It can be observed that all histograms are skewed to be less than the critical values. 


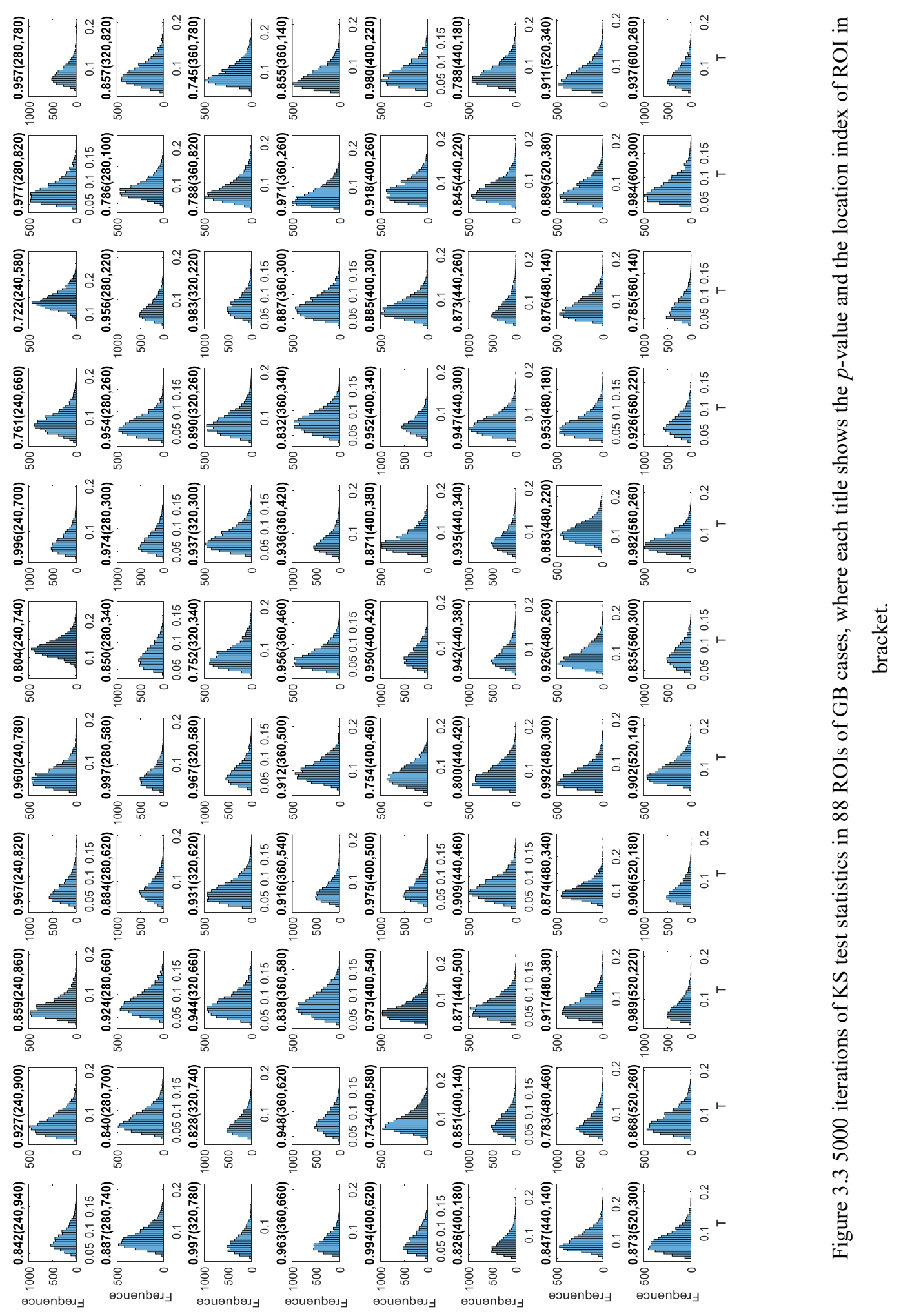


It is not possible to show the Australia case here because of the too large number of ROIs produced. However, the distribution of the $p$-value of all ROIs in GB and AU (Figure 3.4a) obtained from using these two test methods, shows that that the GEV distribution fits well the grid-based areal AMDR series with a $100 \%$ pass of the KS test and more than $97 \%$ for the AD test. For the AMDR time series extracted from the 903 catchments, the GOF results are presented in Figure 3.4b where the GEV distribution can fit well as the $p$-value in the majority is close to 1 with more than $95 \%$ passing the KS and AD tests.
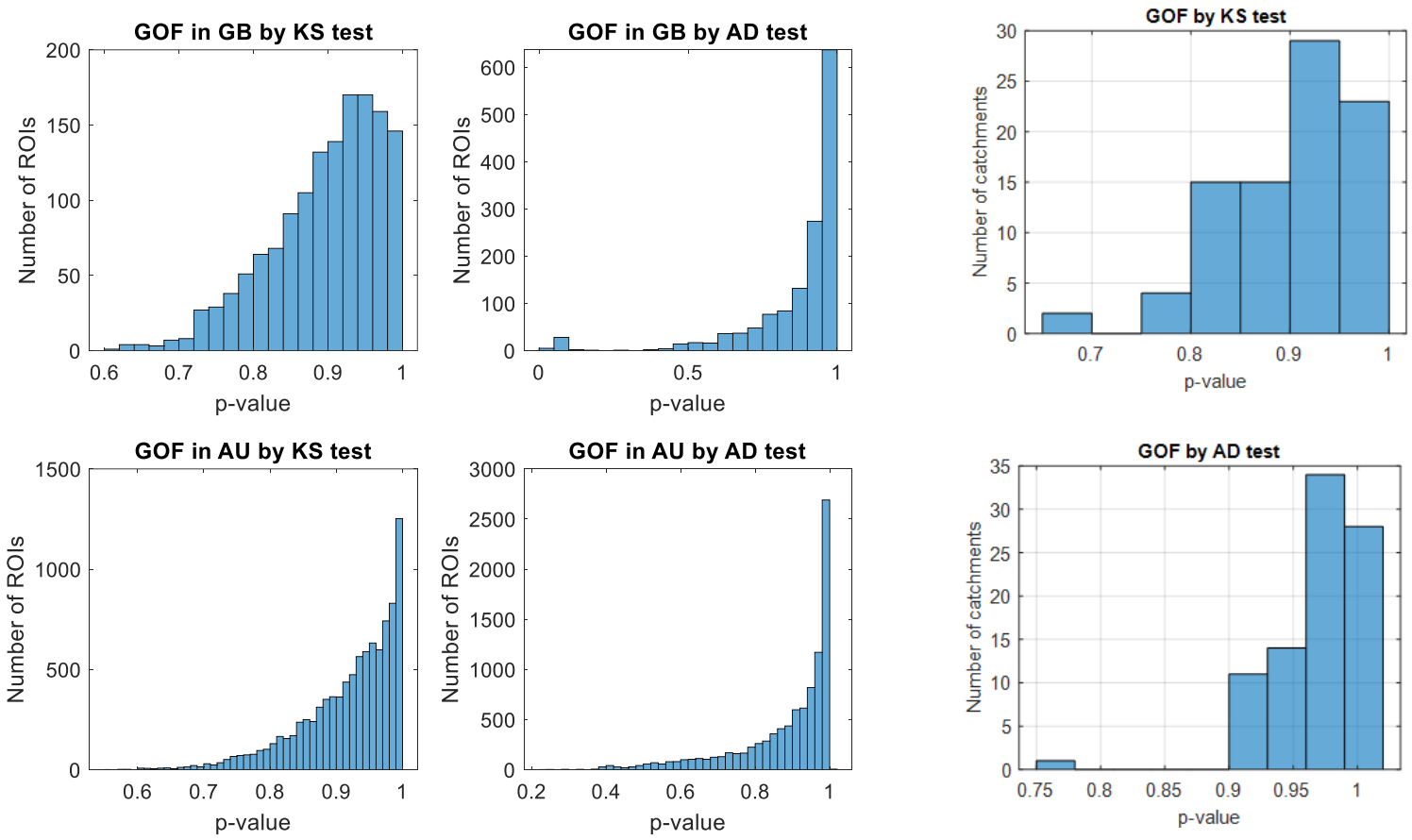

(a)

(b)

Figure 3.4 Histograms of the $p$-value of all ROIs (a) in GB and AU and all catchments (b) in England and Wales by KS and $\mathrm{AD}$ tests.

Figure 3.5 presents the L-moment ratio diagram of L-kurtosis versus L-skewness calculated using the AMDR time series of all ROIs plotted as circles, and the fitted GEV distribution is shown as a blue curve for both cases GB (a) and AU (b). The colours and sizes of circles indicate the geographic location of ROIs, i.e., the easting $x$-index and the northing $y$-index of the ROIs respectively. It can be observed that in both cases, the GEV distribution is an appropriate candidate distribution because its curves properly model the majority of the data 
points displayed. The AMDR in almost all ROIs of the two countries has a large-skewed empirical distribution $(0.1<$ L-skewness of GB $<0.35$ and $0.05<$ L-skewness of AU $<0.4)$. In GB, the majority of the dark-coloured, large-sized circles, indicating the ROIs located in north and west regions, have a relatively medium kurtosis value $(0.15 \sim 0.2)$ while the major ROIs located in south and east regions have both greater kurtosis $(>0.2)$ and skewness $(>0.25)$. In $\mathrm{AU}$, the majority of light-coloured circles (ROIs in the east regions) are located along the GEV curves and have a relatively medium kurtosis. However, ROIs with the same value of Lskewness (indicated by dark-coloured circles) located in the eastern regions have a higher kurtosis than those located in the east. The L-moment ratio diagram helps visualise the comparison between GEV fitted distribution and the characteristics of AMDR themselves, which shows that GEV can fit the majority well.

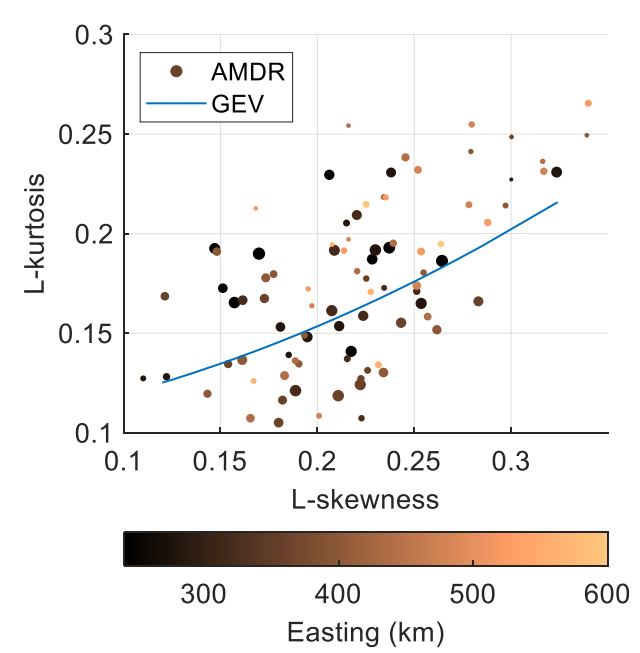

(a)

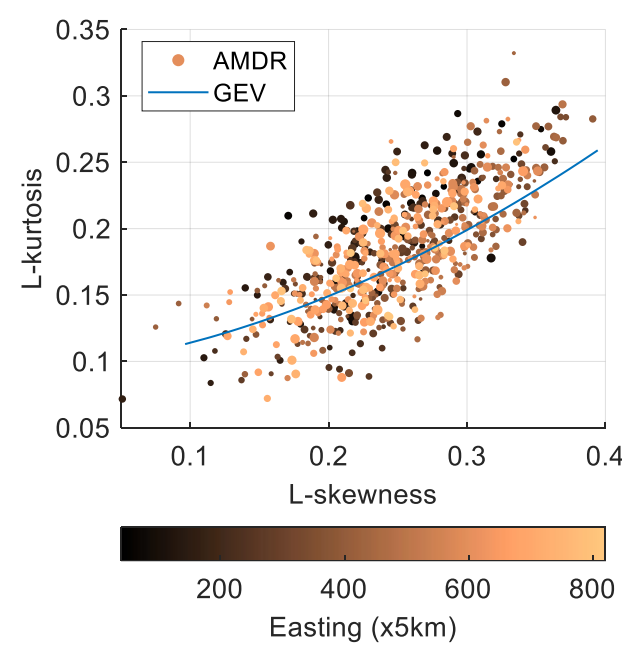

(b)

Figure 3.5 L-moment ratio diagram of L-kurtosis versus L-skewness in GB (a) and AU (b) 


\subsection{Qualitative analysis of the spatial variation of ROI-based study}

The qualitative results of spatial variation of GEV location and scale parameters are described in the following three subsections in respect of three spatial features.

\subsubsection{GEV parameter variation over geographical locations}

Figure 3.6 present the histograms and spatial variations of the three GEV parameters of all ROIs in GB and AU where the following patterns can be clearly identified:

- Most ROIs are in favour of the Frechét type of distribution $(\xi>0)$ and $\xi$ shows insignificant patterns over space but it may be affected by elevation.

- Both $\sigma$ and $\mu$ present a similar spatial pattern where a higher $\mu$ is usually accompanied by a higher $\sigma$.

In GB, the values of $\mu$ and $\sigma$ in the western region, especially in the coastal area, are much larger than those in the east. Such west-east gradient is also strong in the west indicated by the much denser contours. However, there is no remarkable variation from south to north, even though the $\mu$ and $\sigma$ in Scotland are higher. As such, the meridional average is thought to better reveal such an eastward pattern. This meridional spatial pattern can be described as "west high, east low" with an apparently nonlinear variation.

The values of $\mu$ and $\sigma$ in AU have a clear increasing trend from the south-middle zone to the coastal regions. This spatial pattern can be seen as a series of concentric circles. It is also notable that the rapid variations are close to the northeastern coastal regions. For a matter of convenience, the meridional average is also taken for studying the west-east variation in AU. 

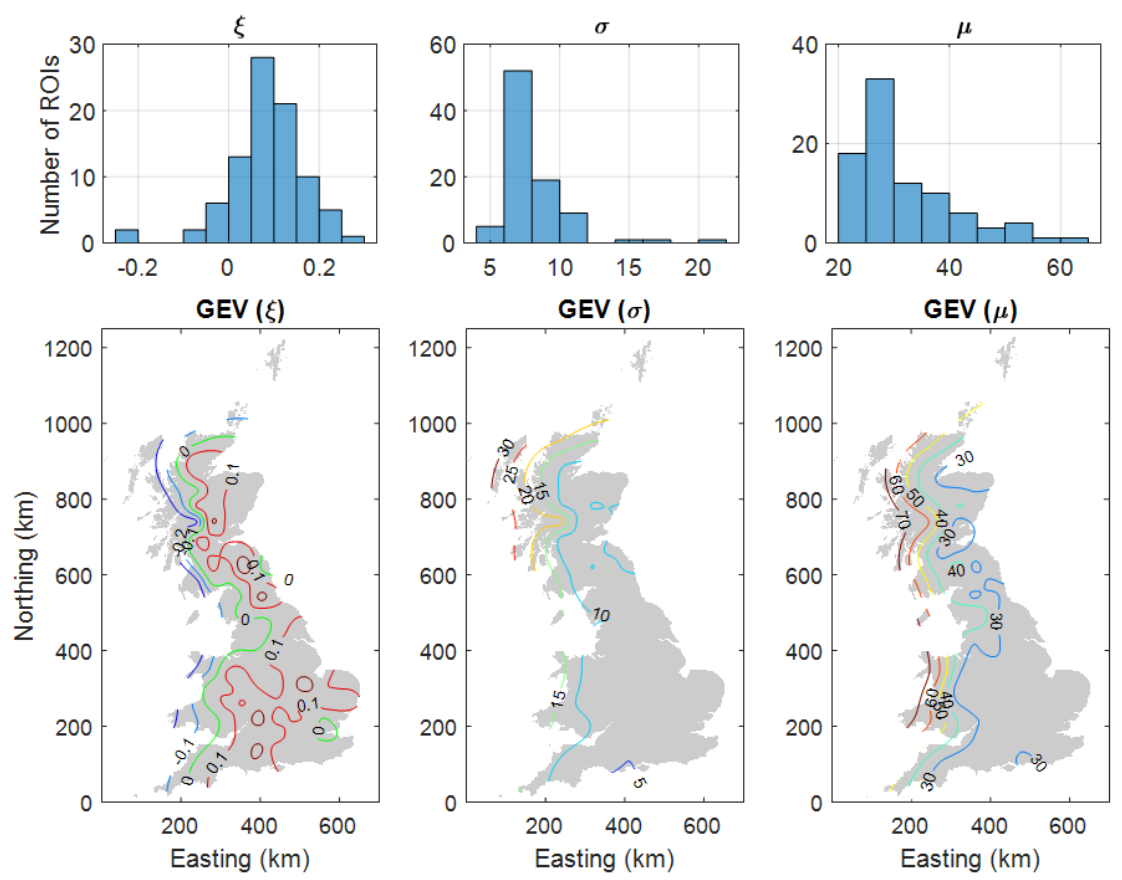

(a)
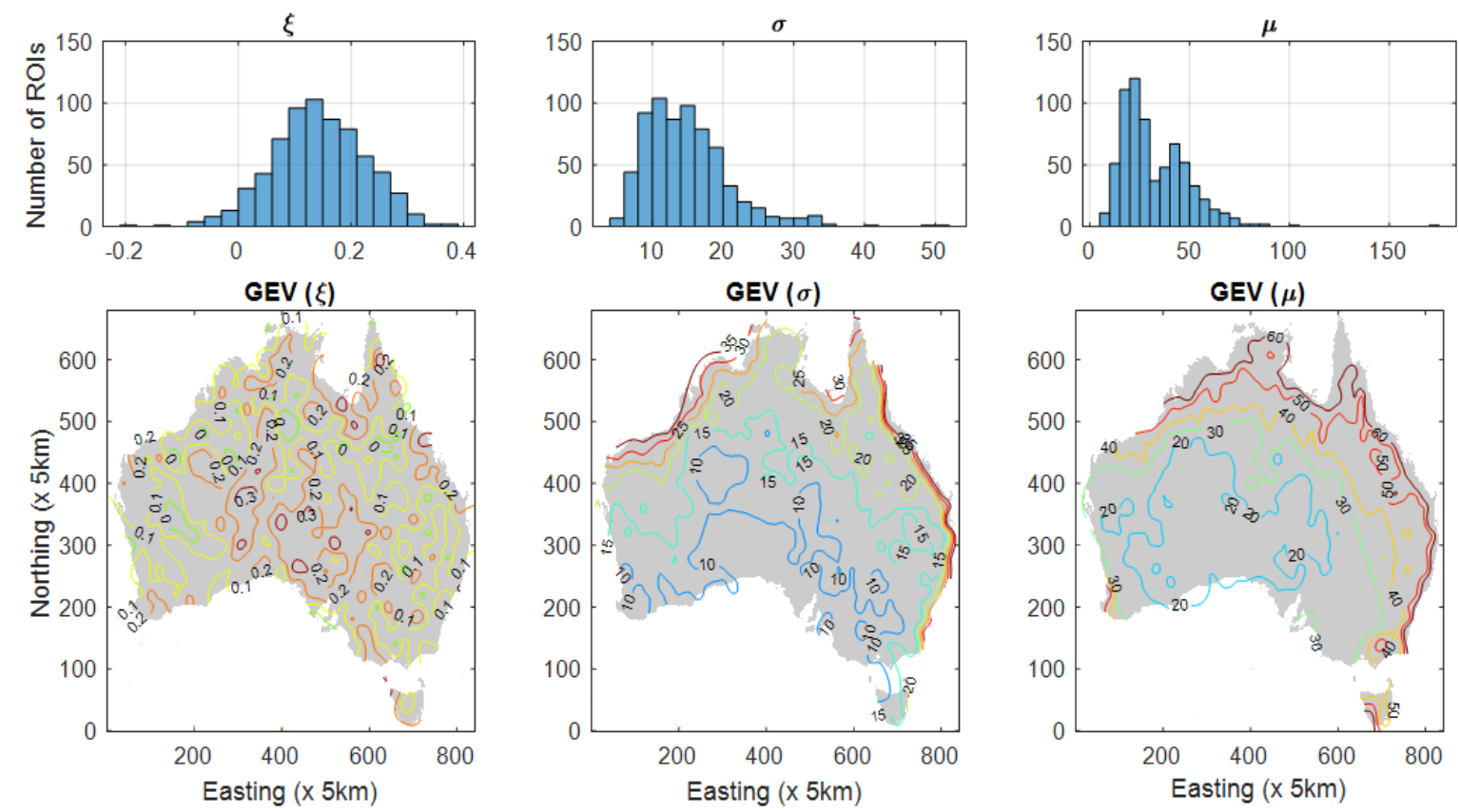

(b)

Figure 3.6 Histograms and spatial variations of the three GEV parameters in GB (a) and AU (b). 


\subsubsection{Variation of GEV parameters with regards to the area size}

Figure 3.7 show the changes of $\mu$ and $\sigma$ of all meridional groups in GB and AU, parameterised by the size of the ROI $\left(s\right.$, in $\left.\mathrm{km}^{2}\right)$. Generally, regardless of their locations, the parameter values are inversely proportional to the sizes of the ROIs.

The decreases in both $\mu$ and $\sigma$ with increased ROI sizes have an important implication: the most frequent AMDR (relating to $\mu$ ) becomes smaller for larger ROI alongside an overall decreased extremity (relating both parameters). Another interesting measure is the rate of such reduction (RR) as the size of ROI increases, which has also shown a clear spatial dependency. In $\mathrm{AU}$, the RR remains low in the central desert zone (e.g., from Easting 300 to $360 \mathrm{~km}$ ), and it increases near the coastal areas where large parameter values are also found. This feature can be explained by the fact that regions having more extreme rainfall (e.g., the outer coastal regions in $\mathrm{AU}$ ) are not only manifested by the higher $\mu$ and $\sigma$; they also have more heterogenous rainfall than those with less extreme rainfall (lower $\mu$ and $\sigma$ ). Therefore, the changes of $\mu$ and $\sigma$ are more sensitive to geographic locations, as revealed by the RR. GB also shows a similar pattern albeit not as remarkable.

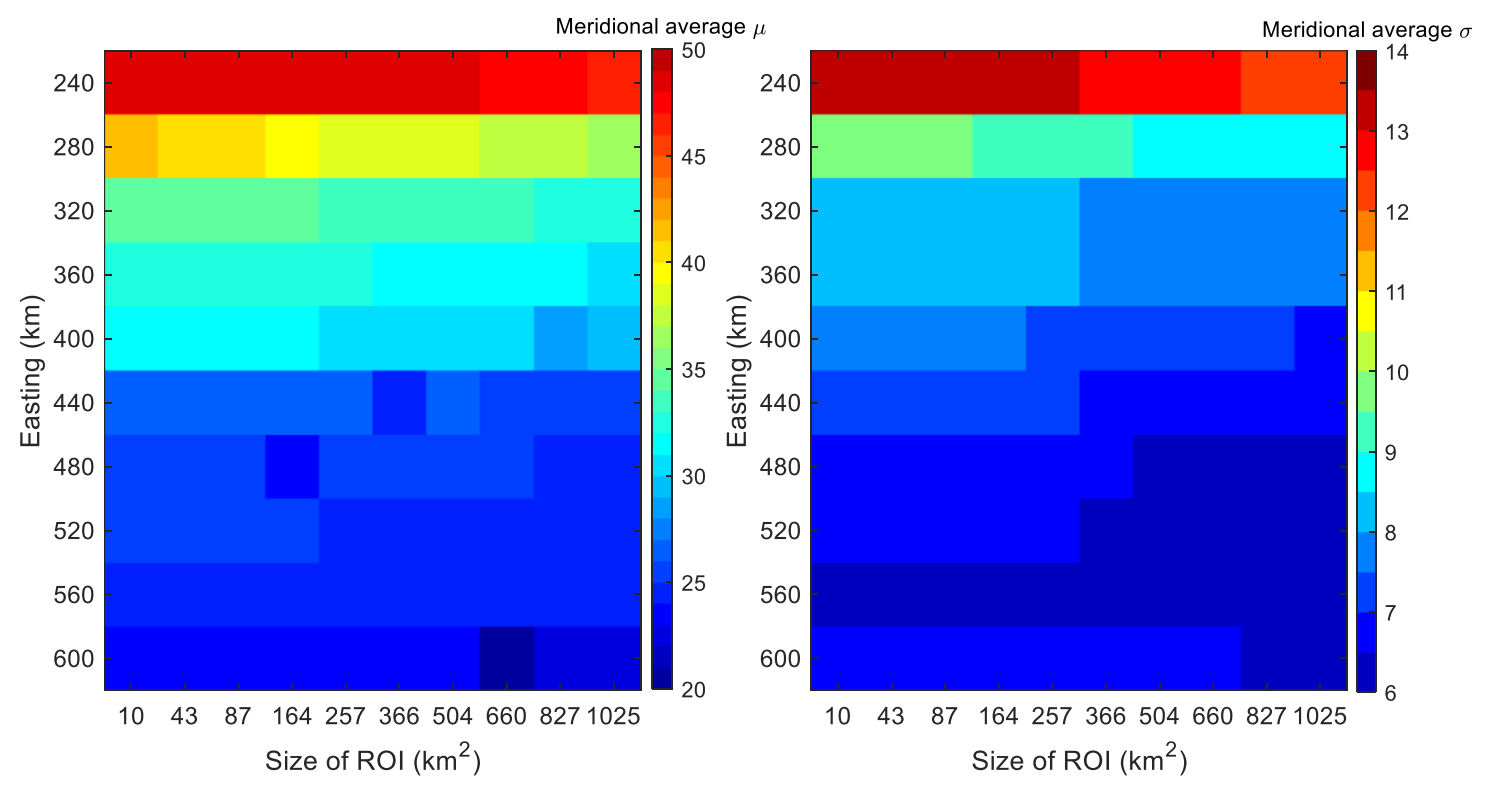

(a) 


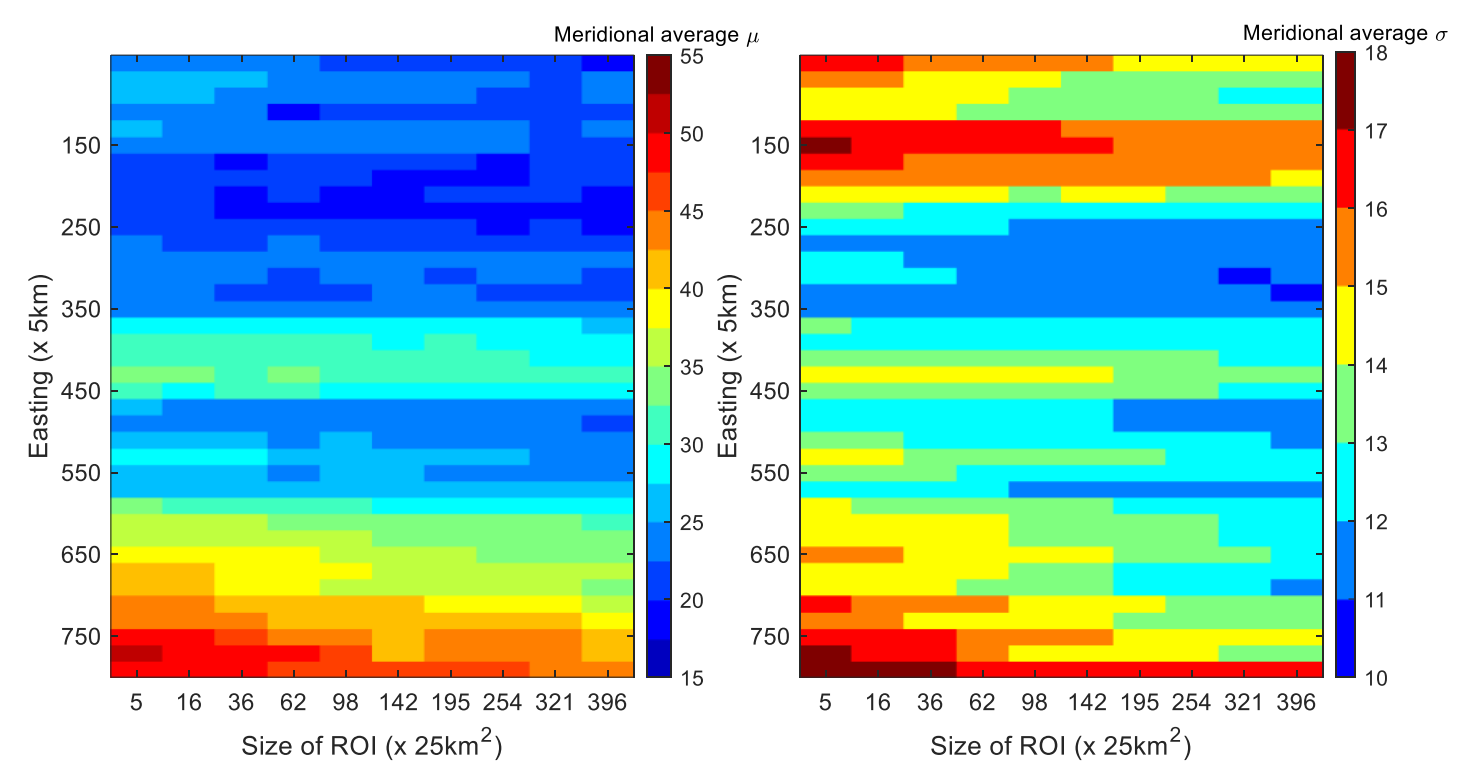

(b)

Figure 3.7 The changes of meridional average location-scale parameters with the ROIs' size in GB (a) and AU (b) shown by colour scale.

\subsubsection{Variation of GEV parameters due to change of ROI shape}

Figure 3.8 presents the changes of $\mu$ and $\sigma$ in GB and AU, parameterised by the ROI shape $(s p)$. The variation of the shape starts from west-east orientated shapes $(s p=0.2)$, gradually growing into more rounded shapes $(s p=1.0)$ and then to more north-south orientated shapes $(s p=5.0)$. By the definition of $s p$, two shapes with reciprocal $s p$ values will have their major dimension swapped, i.e., east-west versus south-north and vice versa.

The result is inspected and summarised as:

- For the majority of the meridional groups, there is little difference between the locationscale parameters of ROIs with reciprocal shapes, e.g., two shapes with $s p$ values of 0.2 and 5.0. This is regarded as a symmetric pattern around $s p=1.0$;

- Generally, the values of $\mu$ and $\sigma$ of ROIs in an elongated shape are smaller than those of the ROIs in more rounded shapes. This indicates that the rounded-shape ROIs have a better chance to capture more rainfall extremes than the elongated ones. It also leads 
to that for the same area size, regions with more regular shapes tend to have more extreme areal rainfall.

- Overall, the effects of ROI shape are not as significant.

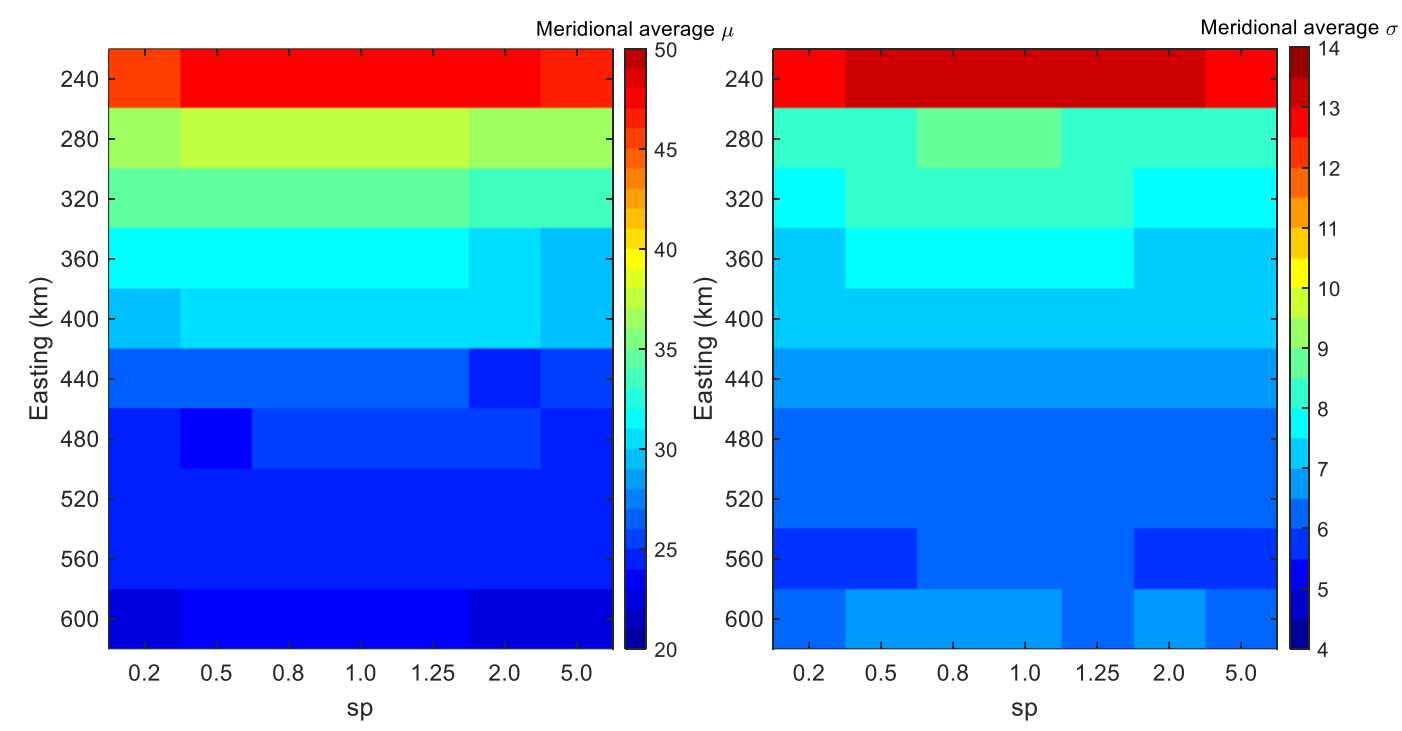

(a)

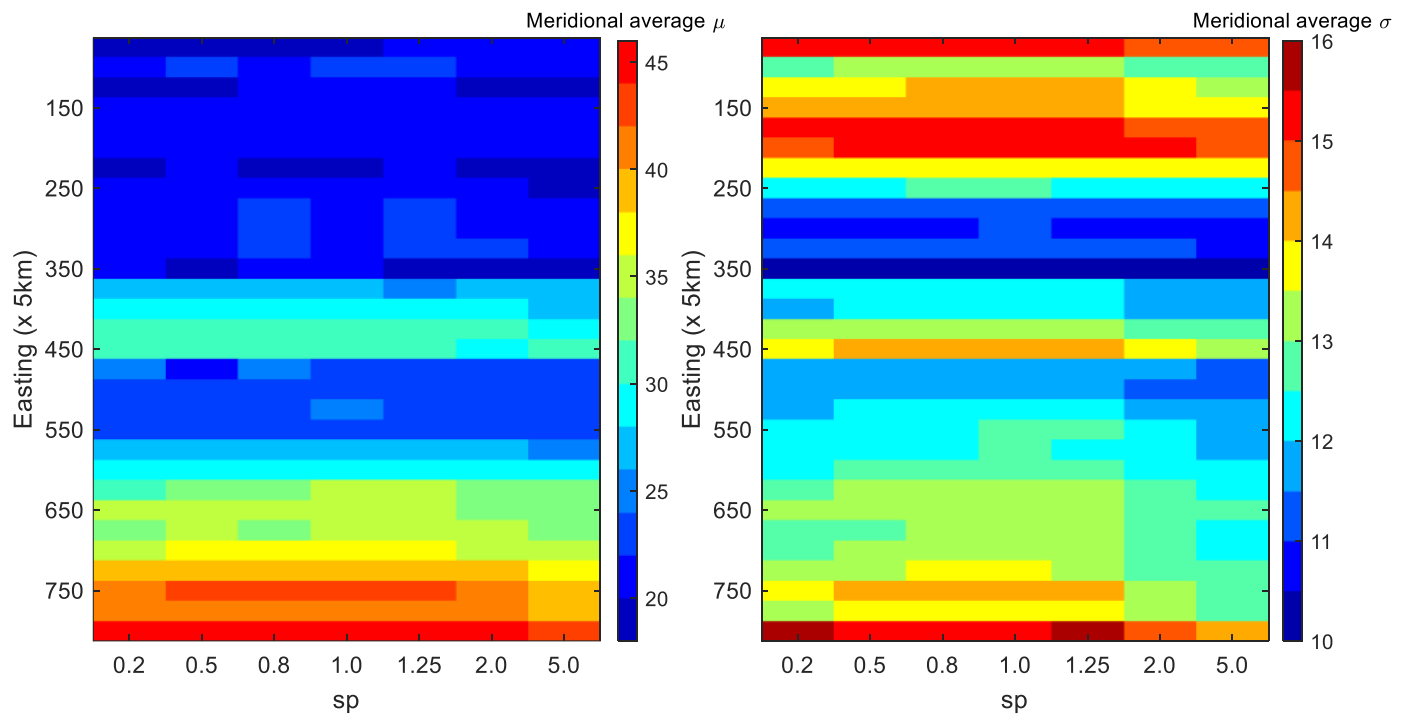

(b)

Figure 3.8 The changes of meridional average location-scale parameters with the ROIs' shape in GB (a) and AU (b) shown in the colour scale. 


\subsubsection{Quantitative modelling of the spatial variation}

The generalised linear models (GLMs) are based on an extension to the classical linear regression model (McCullagh, 1989), and have found many applications in hydrology and meteorology (Coe and Stern, 1982; Stern and Coe, 1984). GLMs have been shown to be effective in incorporating complex structures (Segond et al., 2006). Chandler and Wheater (2002) proposed a GLM-based framework for interpreting historical daily rainfall records and revealing the changes in rainfall occurrence and amount in western Ireland. Many more applications have since followed, e.g., Rashid et al. (2013); Yan et al. (2002); Yang et al. (2005), with good performance reported.

In this study, the two parameters $\mu$ and $\sigma$ which reflect the property of rainfall extremes, show a similar right-skewed gamma distribution (see Figure 3.9), therefore I broadly followed Chandler and Wheater (2002) and propose a GLM with a log-link to describe their spatial variation:

$$
\ln (v)=\boldsymbol{X} \boldsymbol{\beta}
$$

where $v$ is the mean of the distribution; $\boldsymbol{\beta}$ is the estimated vector of coefficients of predictors which are the three spatial properties of the underlying ROIs, i.e., the size $(s)$, location (xindex: $x$ ), and shape $(s p)$ according to the qualitative analysis in Section 3.4.3; and $\boldsymbol{X}$ is the form of combined predictors. 

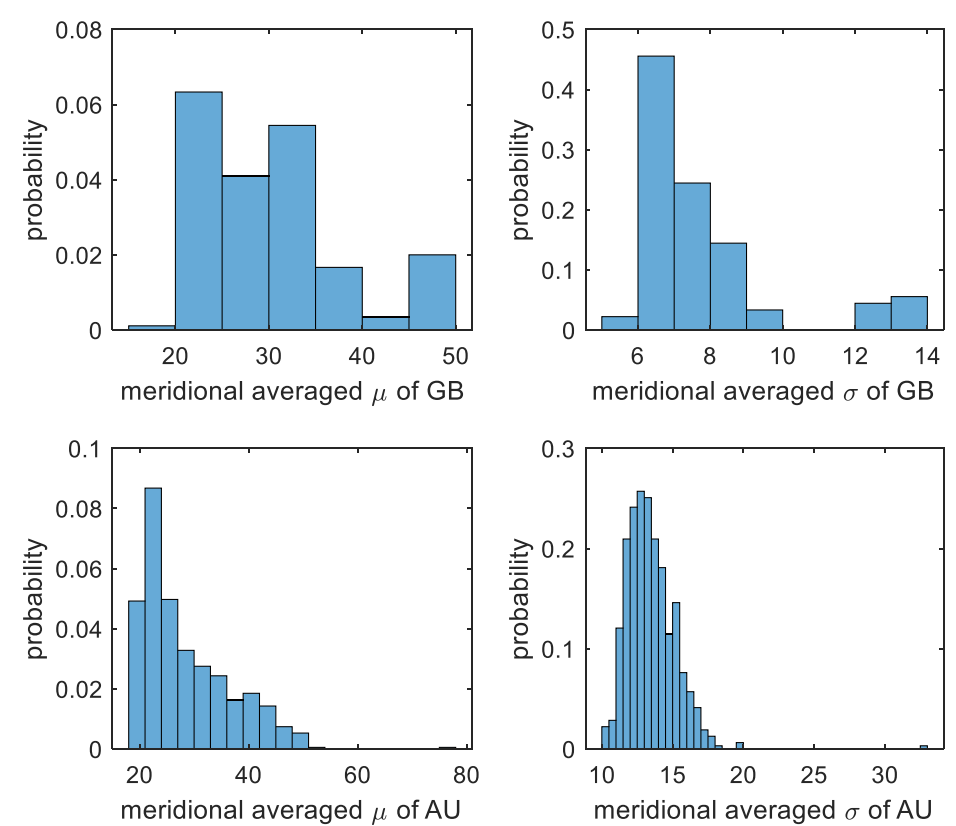

Figure 3.9 Histograms of GEV parameters (meridional averaged $\mu$ and $\sigma$ ) of GB and AU cases.

To get the form of $\boldsymbol{X}$, the fitting of the GLM starts with a simplest form and then successively adds other predictors or their combinations (Chandler and Wheater, 2002; James, 2002). The significance of the newly added predictor or the combination of each attempt is evaluated by calculating the value of log-likelihood at 0.05 insignificance level. The best-fitted form of GLMs is obtained by considering both the log-likelihood and the discrepancy (e.g., root mean squared error, RMSE). All attempts of proposed forms $\boldsymbol{X}$ for two parameters of two cases are shown in Table 3.2.

Table 3.2 All attempts of proposed GLM form $\boldsymbol{X}$ for two parameters of two cases.

\begin{tabular}{|c|c|c|c|c|c|}
\hline \multicolumn{2}{|c|}{ Model No. } & Form $(\boldsymbol{X})$ & $\begin{array}{c}\text { Log } \\
\text { likelihood }\end{array}$ & RMSE & Comments \\
\hline \multirow{4}{*}{$\begin{array}{c}\text { GB } \\
(\mu)\end{array}$} & GM1 & $1+x$ & -401.021 & 2.583 & $x$ is significant \\
\cline { 2 - 6 } & GM2 & $1+x^{2}+x$ & -302.68 & 1.376 & $x^{2}$ is significant \\
\cline { 2 - 6 } & GM3 & $1+x^{3}+x^{2}+x$ & -289.06 & 1.203 & $x^{3}$ is significant \\
\cline { 2 - 6 } & GM4 & $\mathbf{1}+\boldsymbol{x}^{\mathbf{3}}+\boldsymbol{x}^{\mathbf{2}}+\boldsymbol{x}+\boldsymbol{s}$ & $\mathbf{- 2 7 7 . 2 0}$ & $\mathbf{1 . 1 0 1}$ & $s$ is significant \\
\cline { 2 - 6 } & GM5 & $1+x^{3}+x^{2}+x+s^{2}+s$ & -276.18 & 1.091 & $s^{2}$ is insignificant \\
\cline { 2 - 6 } & GM6 & $1+x^{3}+x^{2}+x+s+s p$ & -276.13 & 1.090 & $s p$ is insignificant \\
\hline
\end{tabular}




\begin{tabular}{|c|c|c|c|c|c|}
\hline & GM7 & $1+x^{3}+x^{2}+x+s+x \times s$ & -276.80 & 1.097 & $\begin{array}{c}x \times s \text { is } \\
\text { insignificant }\end{array}$ \\
\hline \multirow{7}{*}{$\begin{array}{l}\mathrm{GB} \\
(\sigma)\end{array}$} & GS1 & $1+x$ & -227.77 & 1.068 & $x$ is significant \\
\hline & GS2 & $1+x^{2}+x$ & -130.17 & 0.632 & $x^{2}$ is significant \\
\hline & GS3 & $1+x^{3}+x^{2}+x$ & -104.36 & 0.500 & $x^{3}$ is significant \\
\hline & GS4 & $1+x^{3}+x^{2}+x+s$ & -89.80 & 0.466 & $S$ is significant \\
\hline & GS5 & $1+x^{3}+x^{2}+x+s^{2}+s$ & -88.14 & 0.464 & $s^{2}$ is insignificant \\
\hline & GS6 & $1+x^{3}+x^{2}+x+s+s p$ & -87.92 & 0.461 & $s p$ is insignificant \\
\hline & GS8 & $1+x^{3}+x^{2}+x+s+x \times s$ & -89.53 & 0.465 & $\begin{array}{c}\quad x \times s \text { is } \\
\text { insignificant }\end{array}$ \\
\hline \multirow{10}{*}{$\begin{array}{c}\mathrm{A} \\
\mathrm{U} \\
(\mu)\end{array}$} & AM1 & $1+x$ & -1739.7 & 4.151 & $x$ is significant \\
\hline & AM2 & $1+x^{2}+x$ & -1608.2 & 3.231 & $x^{2}$ is significant \\
\hline & AM3 & $1+x^{3}+x^{2}+x$ & -1607.6 & 3.217 & $x^{3}$ is insignificant \\
\hline & AM4 & $1+x^{2}+x+s$ & -1567.4 & 2.959 & $S$ is significant \\
\hline & AM5 & $1+x^{2}+x+s^{2}+s$ & -1554.9 & 2.882 & $s^{2}$ is significant \\
\hline & AM6 & $1+x^{2}+x+s^{2}+s+s p$ & -1552.9 & 2.871 & $s p$ is significant \\
\hline & AM7 & $\begin{array}{c}1+x^{2}+x+s^{2}+s+s p^{2} \\
+s p\end{array}$ & -1552.9 & 2.871 & $s p^{2}$ is insignificant \\
\hline & AM8 & $\begin{array}{c}1+x^{2}+x+s^{2}+s+s p \\
+x \times s\end{array}$ & -1552.6 & 2.864 & $\begin{array}{l}\quad x \times s \text { is } \\
\text { insignificant }\end{array}$ \\
\hline & AM9 & $\begin{array}{c}1+x^{2}+x+s^{2}+s+s p \\
+x \times s p\end{array}$ & -1552.9 & 2.871 & $\begin{array}{c}x \times s p \text { is } \\
\text { insignificant }\end{array}$ \\
\hline & AM10 & $\begin{array}{c}1+x^{2}+x+s^{2}+s+s p \\
+s p \times s\end{array}$ & -1552.9 & 2.871 & $\begin{array}{l}\qquad p \times s \text { is } \\
\text { insignificant }\end{array}$ \\
\hline \multirow{10}{*}{$\begin{array}{c}\mathrm{A} \\
\mathrm{U} \\
(\sigma)\end{array}$} & AS1 & $1+x$ & -1207.5 & 1.750 & $x$ is significant \\
\hline & AS2 & $1+x^{2}+x$ & -1025.0 & 1.354 & $x^{2}$ is significant \\
\hline & AS3 & $1+x^{3}+x^{2}+x$ & -1024.0 & 1.349 & $x^{3}$ is insignificant \\
\hline & AS4 & $1+x^{2}+x+s$ & -957.27 & 1.219 & $s$ is significant \\
\hline & AS5 & $1+x^{2}+x+s^{2}+s$ & -941.26 & 1.190 & $s^{2}$ is significant \\
\hline & AS6 & $1+x^{2}+x+s^{2}+s+s p$ & -936.54 & 1.183 & $s p$ is significant \\
\hline & AS7 & $\begin{array}{c}1+x^{2}+x+s^{2}+s+s p^{2} \\
+s p\end{array}$ & -936.31 & 1.183 & $s p^{2}$ is insignificant \\
\hline & AS8 & $\begin{array}{c}1+x^{2}+x+s^{2}+s+s p \\
+x \times s\end{array}$ & -929.80 & 1.170 & $x \times s$ is significant \\
\hline & AS9 & $\begin{aligned} 1+x^{2}+x+s^{2} & +s+s p \\
& +x \times s+x \times s p\end{aligned}$ & -928.64 & 1.167 & $\begin{array}{c}\quad x \times s p \text { is } \\
\text { insignificant }\end{array}$ \\
\hline & AS10 & $\begin{aligned} 1+x^{2}+x+s^{2} & +s+s p \\
& +x \times s+s p \times s\end{aligned}$ & -929.80 & 1.169 & $\begin{array}{l}\quad s p \times s \text { is } \\
\text { insignificant }\end{array}$ \\
\hline
\end{tabular}

To specify the GLMs, the GLM for capturing the meridionally averaged $\mu$ and $\sigma$ for GB case is called "GM" and "GS" respectively and for AU case is "AM" and "AS". There are several GLMs attempted with each attempt adding new terms and the optimal form of GLMs is 
obtained by obtaining the maximum log-likelihood (LL) and the smallest discrepancy (e.g., root mean squared error) with all terms being significant (i.e., the $p$-value is less than critical level 0.05 ). Figure 3.10 shows the process of testing and selection of the best-fitted GLMs, i.e., GM4, GS4, AM6 and AS8.
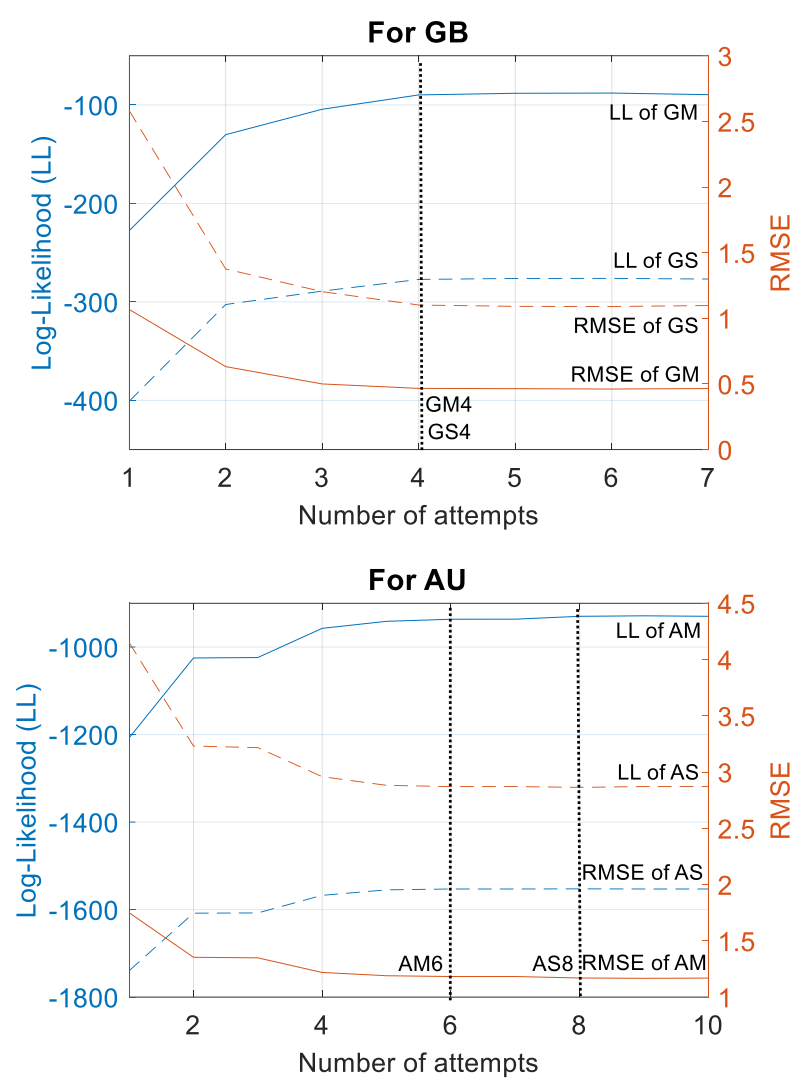

Figure 3.10 The selection process for the optimal structure of GLM for meridionally averaged $\mu$ and $\sigma$ in GB and AU.

According to the values of LL and RMSE, the best-fitted GLMs are model GM4 and GS4 for GB case and model AM6 and AS8 for AU case with the optimum form of GLM models are identified as follows:

$$
\begin{aligned}
& \text { For } \mu_{G B}:\left(1+x+s+x^{2}+x^{3}\right) \boldsymbol{\beta}_{\boldsymbol{\mu}_{G B}} \\
& \text { For } \sigma_{G B}:\left(1+x+s+x^{2}+x^{3}\right) \boldsymbol{\beta}_{\sigma_{G B}}
\end{aligned}
$$




$$
\begin{gathered}
\text { For } \mu_{A U}:\left(1+x+s+s p+x^{2}+s^{2}\right) \boldsymbol{\beta}_{\boldsymbol{\mu}_{A U}} \\
\text { For } \sigma_{A U}:\left(1+x+s+s p+x^{2}+s^{2}+x \times s\right) \boldsymbol{\beta}_{\sigma_{A U}}
\end{gathered}
$$

where the subscripts of $\boldsymbol{\beta}$ GB and AU refer to the study area in question. A maximum likelihood estimator (McCullagh, 1989) was employed for obtaining $\boldsymbol{\beta}$ s which are shown in with their $p$-values shown in brackets. Except the intercept, the coefficient of the term $x$ (indicating the location of ROIs by the easting index) is the (absolute) maximum polynomial factor.

Table 3.3 Coefficients of four GLMs and their $p$-values (shown in brackets).

\begin{tabular}{|c|c|c|c|c|c|c|c|c|}
\hline $\begin{array}{c}\text { GLM } \\
\text { For }\end{array}$ & $\beta_{0}$ & $\begin{array}{c}x \\
(100 \mathrm{~km})\end{array}$ & $\begin{array}{c}x^{2} \\
(100 \mathrm{~km})\end{array}$ & $\begin{array}{c}x^{3} \\
(100 \mathrm{~km})\end{array}$ & $\begin{array}{c}s \\
\left(100 \mathrm{~km}^{2}\right)\end{array}$ & $\begin{array}{c}s^{2} \\
\left(100 \mathrm{~km}^{2}\right)\end{array}$ & $s p$ & $\begin{array}{c}x \times s \\
(100 \mathrm{~km} \\
\times 100 \\
\mathrm{~km}^{2}\end{array}$ \\
\hline$\mu_{G B}$ & $\begin{array}{c}5.98 \\
(7.9 \mathrm{e}-83)\end{array}$ & $\begin{array}{c}\mathbf{- 1 . 3 6} \\
(\mathbf{1 . 2 e - 2 0})\end{array}$ & $\begin{array}{c}0.24 \\
(3.4 \mathrm{e}-12)\end{array}$ & $\begin{array}{c}-1.46 \mathrm{e}-2 \\
(2.4 \mathrm{e}-08)\end{array}$ & $\begin{array}{c}-5.62 \mathrm{e}-3 \\
(1.1 \mathrm{e}-06)\end{array}$ & - & - & - \\
\hline \multirow{2}{*}{$\sigma_{G B}$} & $\begin{array}{c}5.85 \\
(5.7 \mathrm{e}-60)\end{array}$ & $\mathbf{- 2 . 2 8}$ & 0.44 & $-2.92 \mathrm{e}-2$ & $-8.69 \mathrm{e}-3$ & - & - & - \\
\hline \multirow{2}{*}{$\mu_{A U}$} & 3.23 & $\mathbf{( 1 . 5 e - 2 6 )}$ & $(5.6 \mathrm{e}-19)$ & $(2.4 \mathrm{e}-14)$ & $(9.3 \mathrm{e}-08)$ & - & - & - \\
\hline \multirow{2}{*}{$\sigma_{A U}$} & 2.90 & $\mathbf{- 0 . 0 6}$ & $1.82 \mathrm{e}-2$ & - & $-3.34 \mathrm{e}-3$ & $2.20 \mathrm{e}-5$ & -0.0092 & - \\
& $(0)$ & $\mathbf{( 1 . 6 e - 1 0 )}$ & $(1.3 \mathrm{e}-61)$ & - & $(2.8 \mathrm{e}-12)$ & $(1.3 \mathrm{e}-05)$ & $(0.046)$ & - \\
\hline
\end{tabular}

The GLMs are further visualised in Figure 3.11 where the previously demonstrated, qualitative properties, are readily reproduced. For example, the spatial changes of the two GEV parameters are "west high, east low" in GB whereas they are "centre low, outer coastal regions high" in AU; the parameters get smaller as the size of ROI increases. However, the RR, which can be interpreted as the vertical distance between curves, is more uneven in AU, which means that the reduction on most frequent rainfall $(\mu)$ and occurrence probability of extremes $(\sigma)$ is more spatially dependent and area-oriented compared with GB. Moreover, ROI shape is significant in the AU case where different $\mu$ or $\sigma$ values are observed in the east-west-orientated elongated shapes $(s p=0.2)$ and the rounded ones $(s p=1.0)$ and the difference tends to decrease for lager $\mu$ and $\sigma$. In comparison, the two GEV parameters in the north-south-orientated ROIs $(s p=5.0)$ are also smaller than those in the east-west-orientated and rounded ones, which can 
be explained as that in AU the north-south variation is in general smaller than that of the eastwest direction.

This fitted GLMs help to reveal the following intriguing features regarding the spatial variation of the two parameters:

1) In GB, both the meridionally-averaged $\mu$ and $\sigma$ have a nonlinearly-eastwarddecreasing pattern, and the RR is stronger in the west $\left(\Delta \mu_{G B}=-0.22 / \mathrm{km} ; \Delta \sigma_{G B}=\right.$ $-7.50 / 100 \mathrm{~km})$ and gradually reduces to the east $\left(\Delta \mu_{G B}=-0.02 / \mathrm{km} ; \Delta \sigma_{G B}=\right.$ $-0.30 / 100 \mathrm{~km})$. An almost-linearly-decreasing pattern with the increase of ROI size $\left(\Delta \mu_{G B}=-0.02 / 100 \mathrm{~km}^{2}\right.$ and $\left.\Delta \sigma_{G B}=-0.08 / 100 \mathrm{~km}^{2}\right)$ is shown and such RRs are not affected by geographic locations. However, they do not appear to be dependent on the ROI shape $(s p)$.

2) In $\mathrm{AU}$, the spatial changes of the meridionally-averaged $\mu$ and $\sigma$ are nonlinear with respect to both the ROI location and size. The $\mu$ diminishes slowly from the west coasts to the south-middle zone $\left(\Delta \mu_{A U}=-0.05 / 500 \mathrm{~km}\right)$ then increases faster and faster to the east coasts (largest $\Delta \mu_{A U}=+6.70 / 500 \mathrm{~km}$ ); The $\sigma$ shows a "centre low, outer (coastal regions) high" pattern where the changing rate near the centre $\left(\left|\Delta \sigma_{A U}\right|=\right.$ $0.05 / 500 \mathrm{~km})$ is lower than the coastal regions $\left(\left|\Delta \sigma_{A U}\right|=1.40 / 500 \mathrm{~km}\right)$. Unlike GB, $s p$ plays a more significant role and diminishes both parameters ( $\Delta \mu_{A U}=$ $\left.-0.9, \Delta \sigma_{A U}=-0.5\right)$ when the ROI shape changes from the west-east-orientated ( $s p=$ $0.2,0.5)$ to north-south-orientated $(s p=5.0,2.0)$. It means that the north-south variation in $\mathrm{AU}$ is in general smaller than that of the east-west direction. Besides, the combined term $(x \times s)$ is significant, which means that the RR of $\sigma$ with respect to ROI size varies at different geographic locations. It is manifested by the uneven vertical gaps between contours in the right panel of Figure 3.11b. 

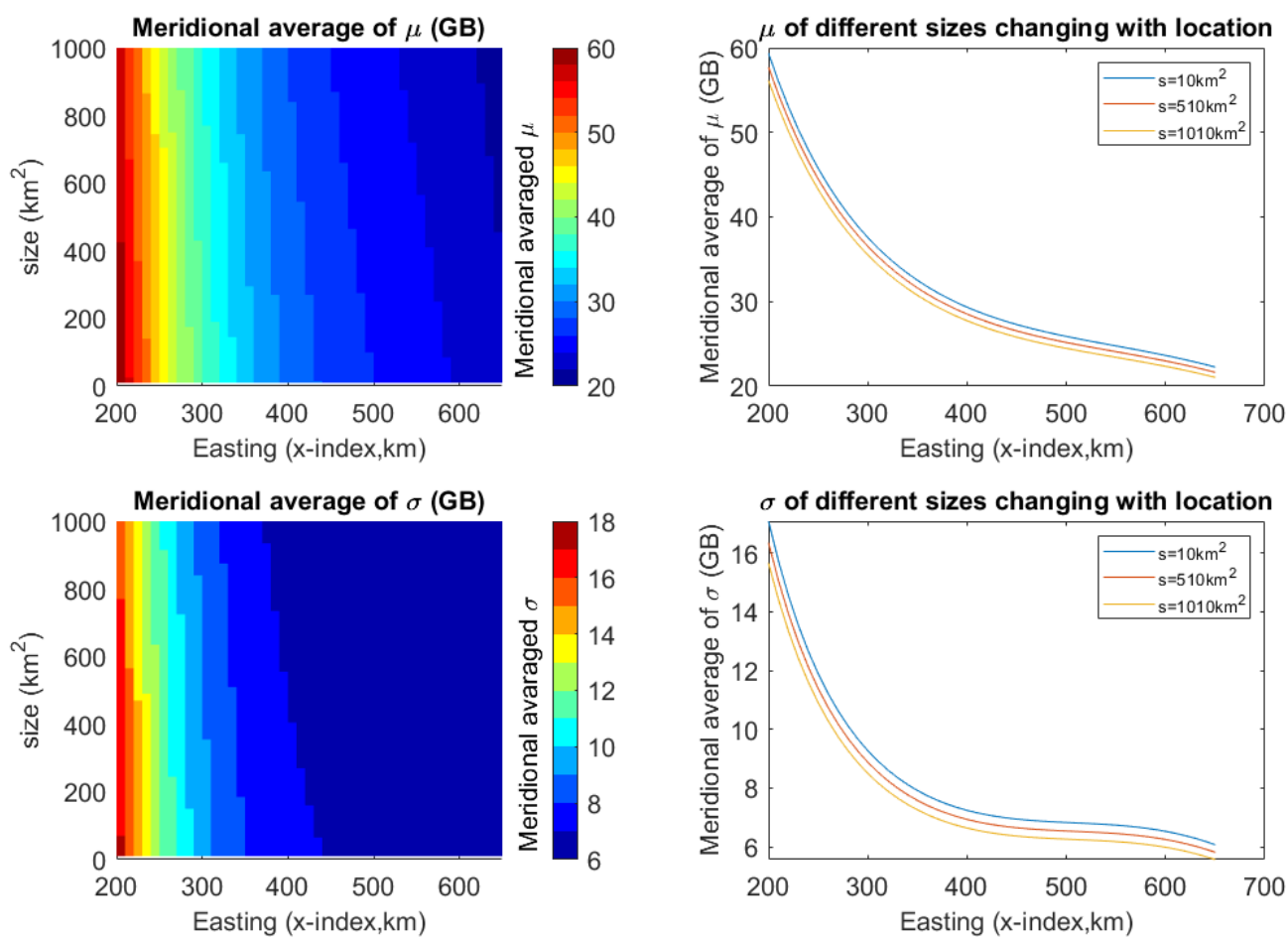

(a)
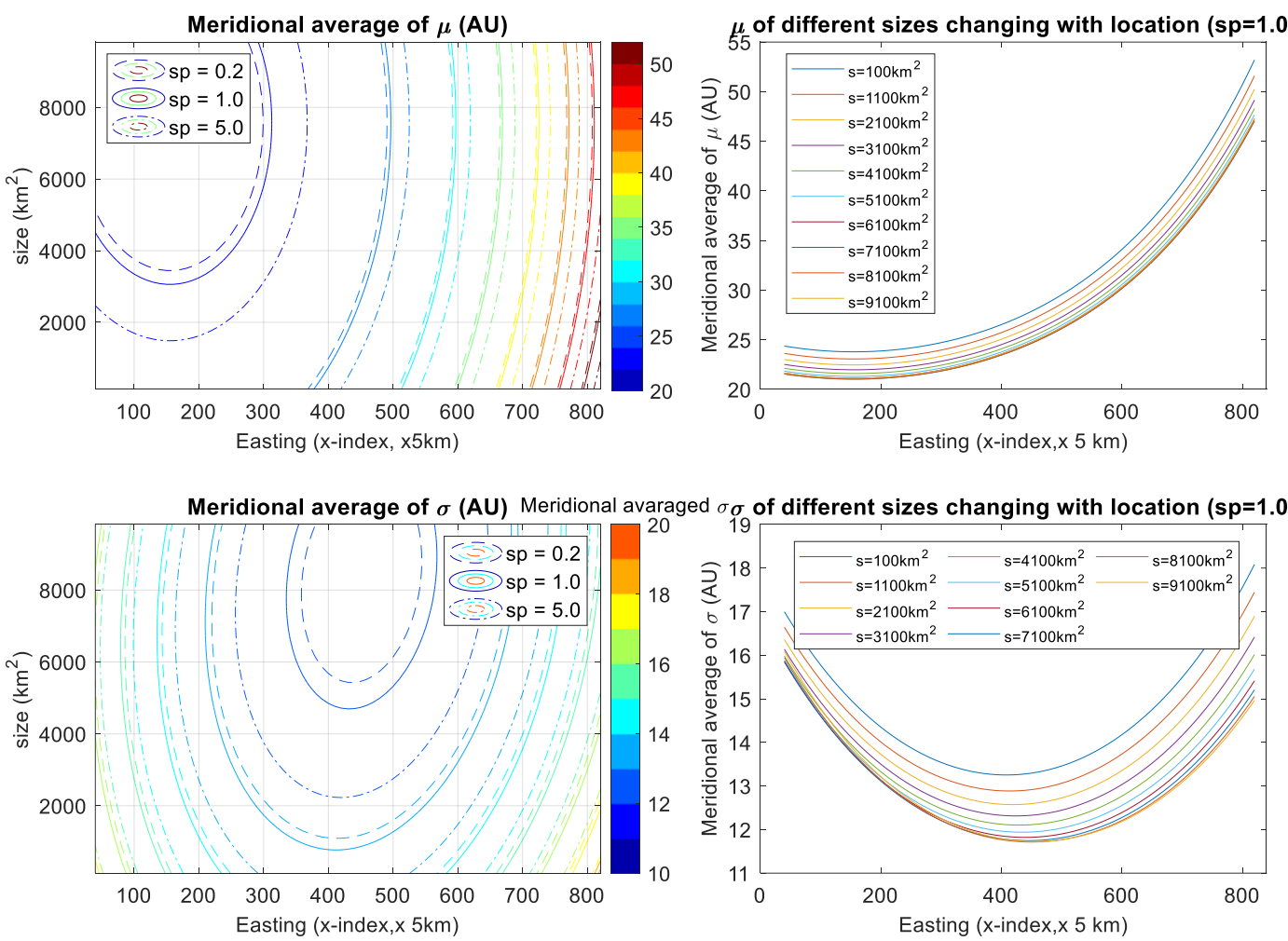

(b) 
Figure 3.11 Visualisation of the GLMs fitted to the meridional average GEV $\mu$ and $\sigma$ parameters as a colourscale plot for GB (a) and a contour plot for AU (b) whose contours are picked up at the same stops of the values and their changes with respect to the geographic location and size.

The performance of the GLMs is evaluated by comparing the parameter values modelled by the GLMs and those from the originally fitted GEVs; as well as by conducting a residual analysis (McCullagh, 2018; Pierce and Schafer, 1986; Wang, 1987).

The GLMs for both cases perform well (see Figure 3.12a). The GB case has slight underestimations for some large values that appear in the western coastal region; and for the AU case, some overestimation happens for the small values which are located in the middlesouth dry zone. The GLM model probability structure is checked by the normal quantile plot (see Figure 3.12b) of the residuals, where a theoretical normal distribution is shown on the $x$ axis compared with the residual quantiles on the $y$-axis. If the probability assumption (i.e., gamma assumption) is correct, all residuals would have the same distribution which is an approximate normal distribution. It can be observed that the distribution of the residuals of the four GLMs is symmetric with two flat sides. Generally, the approximation fits well except for the upper side which represents only $0.9 \%$ of the total data points. In view of the research aims, this is considered to be acceptable. 

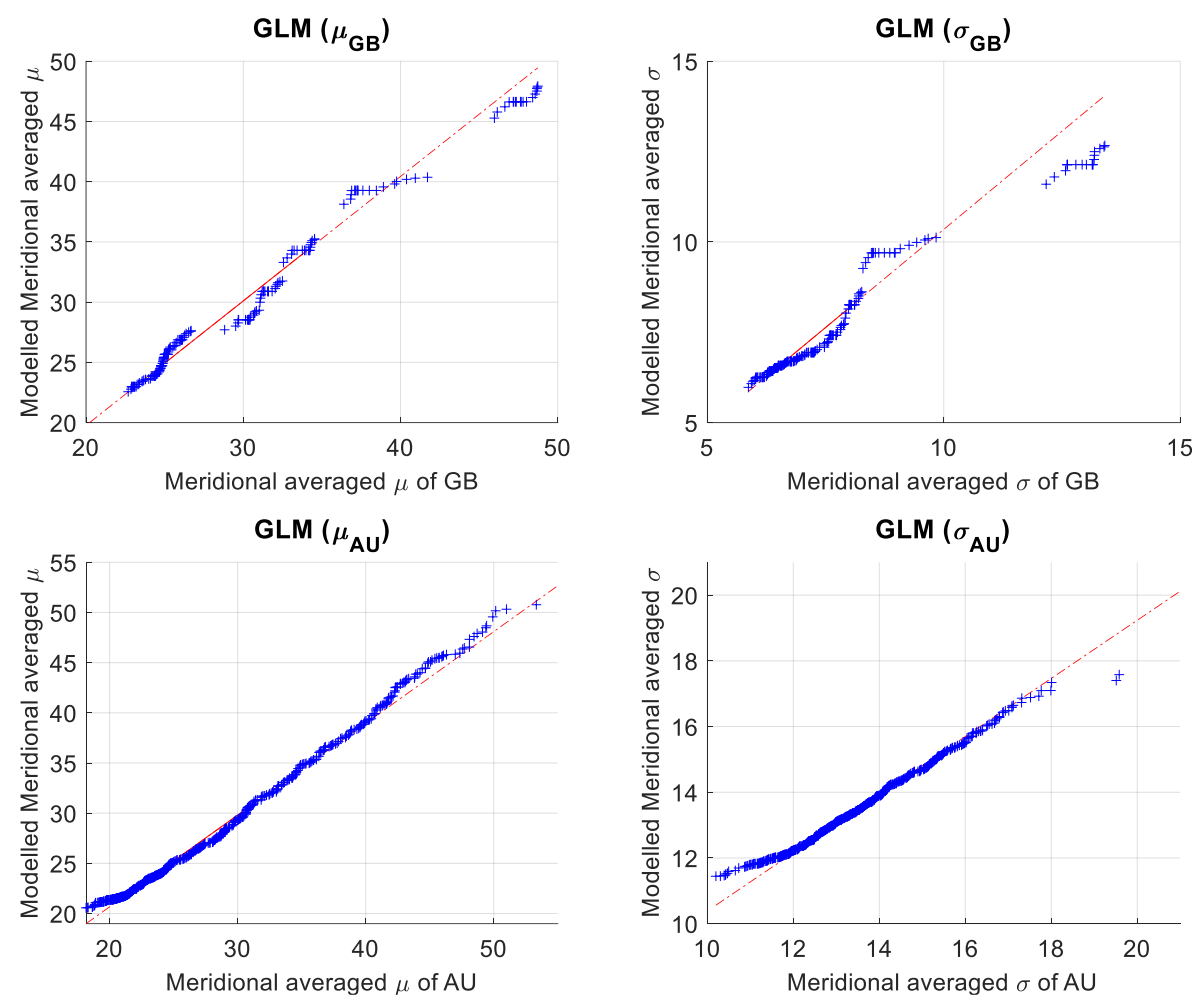

(a)
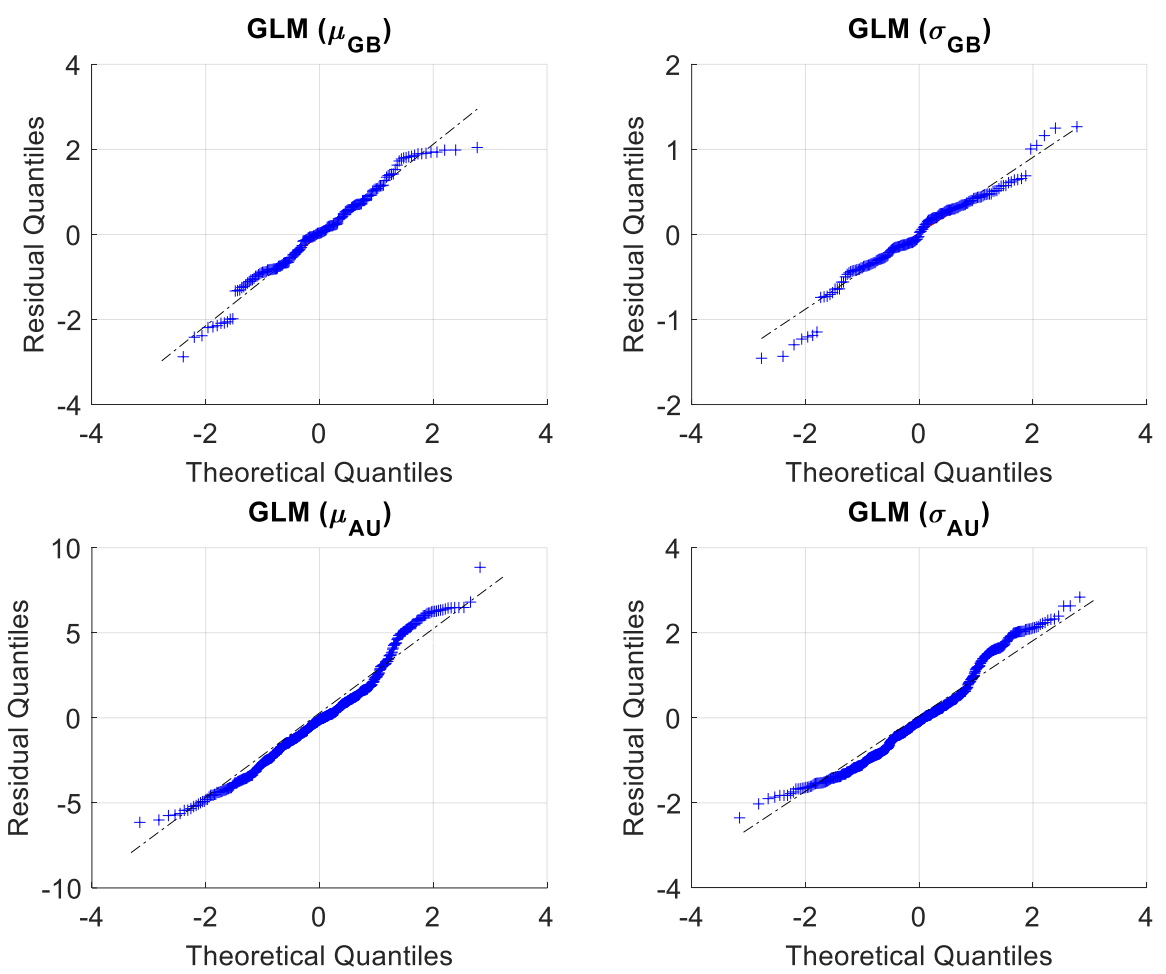

(b) 
Figure 3.12 A scatter plot (a) and a normal quantile plot (b) for revealing the difference between the actual GEV parameters and the modelled GEV parameters.

Meanwhile, the 10-fold cross-validation was used by random partitioning the GEV parameters to test the four GLMs. The average Nash-Sutcliffe efficiency (NSE) coefficient across all partitions of each GLM was reported as $0.97,0.89,0.86$ and 0.68 respectively (shown in Figure 3.13). NSE nearer to 1 means the model with more predictive skill.
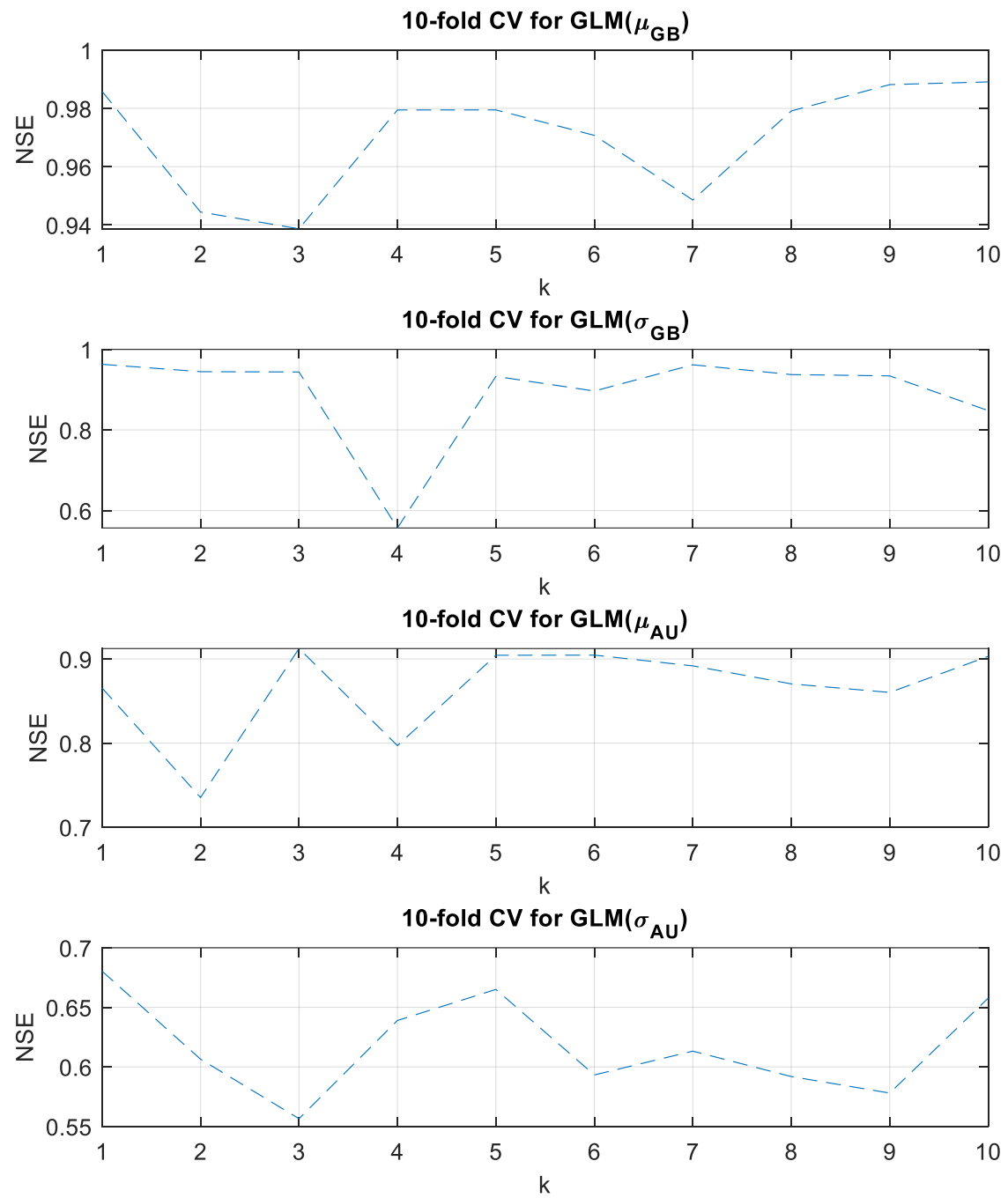

Figure 3.13 Nash-Sutcliffe efficiency (NSE) coefficient of 10-fold cross-validation (CV) method for evaluating the performance of four GLMs. 
In order to reveal whether the GEV parameters $\mu$ and $\sigma$ in the adjacent ROIs are interdependent to a certain distance, i.e., checking the locative continuity, (semi-) variograms are fitted to the data using a widely adopted Spherical Model. And the variogram is calculated by:

$$
\gamma(h)=\frac{1}{2 N(h)} \sum_{i=1}^{N(h)}\left(Z_{l i}-Z_{l i+h}\right)^{2}
$$

where $N(h)$ is the number of pairs of ROI locations at distance $h$ apart and $Z_{l i}$ indicates the value at the location $i$.

The results are presented in Figure 3.14. The meridionally averaged $\mu$ and $\sigma$ in GB, which were estimated by the GEV and fitted by the GLMs respectively, show very little difference on locative continuity (range, sill and nugget). For the distance between the adjacent ROIs less than around $200 \mathrm{~km}$, both $\mu$ and $\sigma$ are specially related. It indicates that the fitted GLM for the GB case is able to reproduce the spatial coherence of the two GEV parameters. For the AU case, similar results are found but with a distance threshold around $400(\times 5 \mathrm{~km})$. There is a difference in the sills when comparing the $\sigma$ parameter from the GLM and the GEV. 


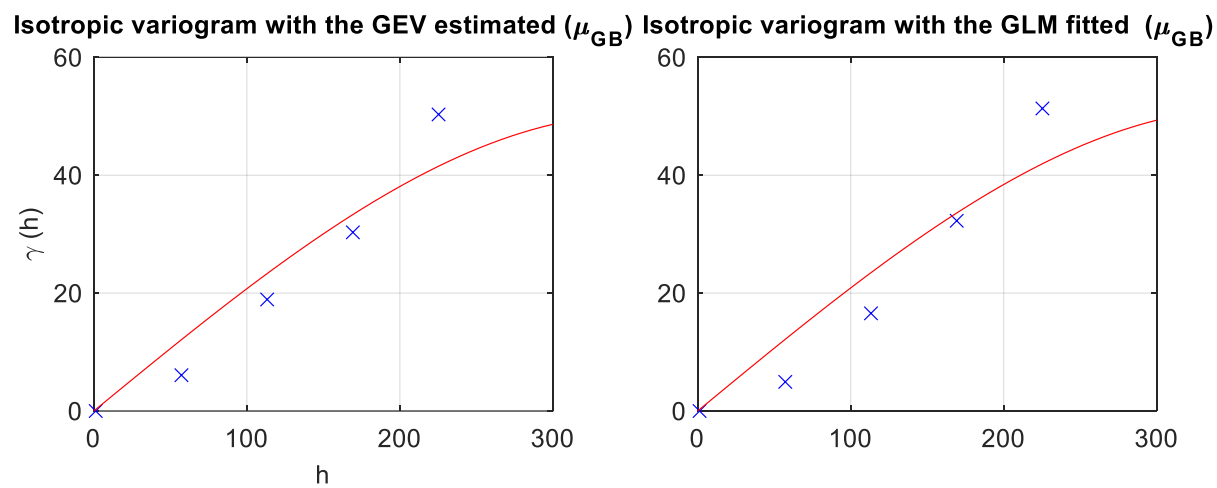

Isotropic variogram with the GEV estimated $\left(\sigma_{\mathrm{GB}}\right)$ Isotropic variogram with the GLM fitted $\left(\sigma_{\mathrm{GB}}\right)$
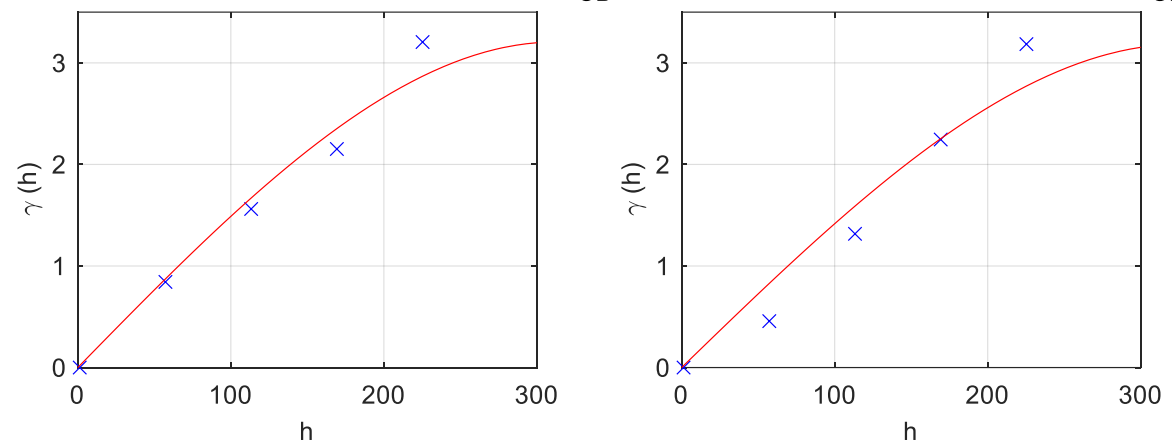

(a)

Isotropic variogram with the GEV esitmated $\left(\mu_{\mathrm{AU}}\right)$ Isotropic variogram with the GLM fitted $\left(\mu_{\mathrm{AU}}\right)$
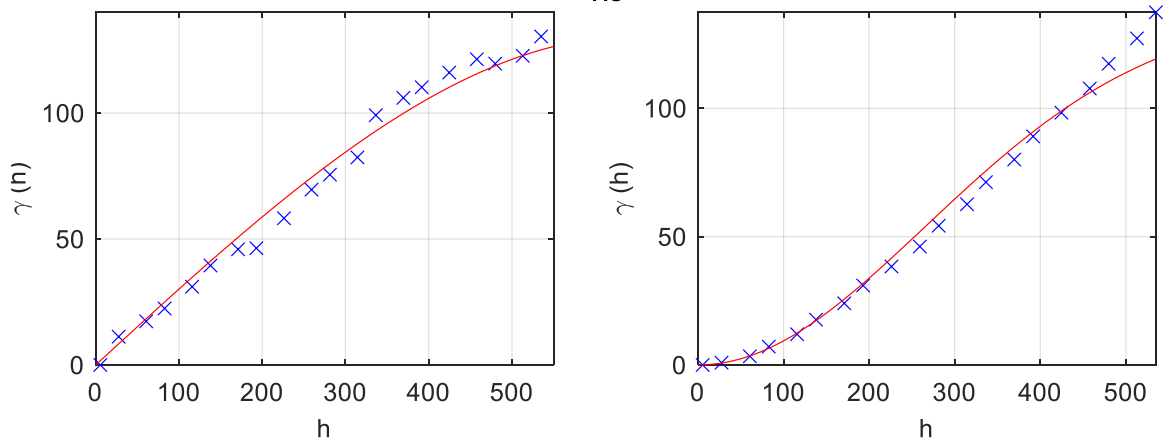

Isotropic variogram with the GEV esitmated $\left(\sigma_{\mathrm{AU}}\right)$ Isotropic variogram with the GLM fitted $\left(\sigma_{\mathrm{AU}}\right)$
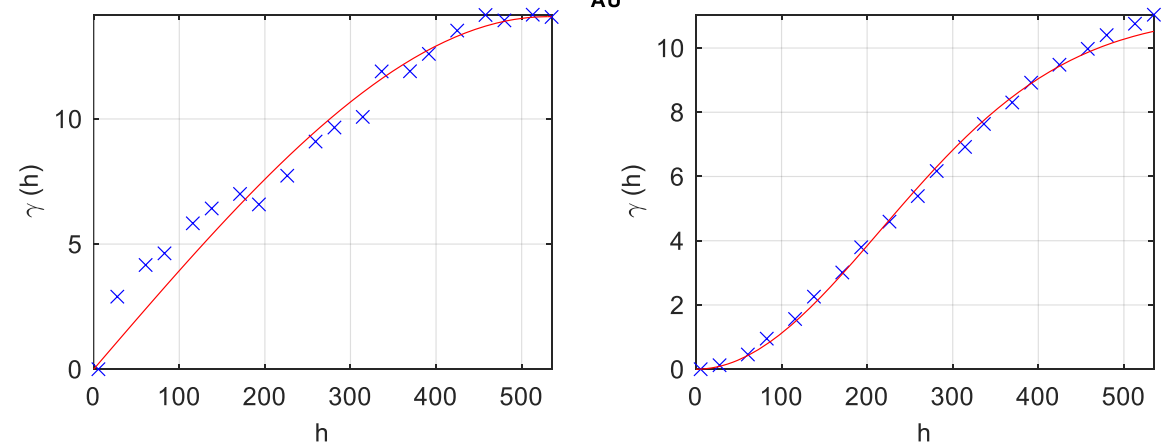

(b) 
Figure 3.14 The variograms for evaluating spatial continuity of GEV parameters $\mu$ and $\sigma$ estimated by GEV and GLM models of GB (a) and AU (b) cases fitted by Spherical model.

These quantitative findings regarding the spatial variation of the GEV parameters have two important implications to many downstream applications of the areal rainfall maxima, e.g., flood risk management (FRM). For one aspect, the traditional approach in FRM makes use of point rainfall maxima to represent the areal one (catchment or a predefined area), where a scaling factor is involved. This simplistic treatment ignores the complexity in spatial distribution, nor can it account for the interplay of the size, location as well as the shape of the area in question, as revealed above. For another, the overall quantification of the spatial variation of the GEV parameters (hence, the return values) makes it possible to study the FRM at the country level as a single entity instead of looking at individual regions with isolations. It also helps to gain insights into how large scale hydroclimatic (rainfall) variation can affect the local FRM, which is very important for studying FRM under climate change impact.

\subsection{Qualitative analysis on the spatial variation of catchment- based study}

\subsubsection{Spatial features of catchments and simulation results of GEV model}

The subfigures $a, b, c$ and $d$ of Figure 3.15 present the spatial distribution of catchments with respect to their spatial features, i.e., elevation $H$, size $S$, orientation angle $\omega$ and shape $s p$. The highest elevation is observed in the Scottish Highland, generally more than 800 metres. Then North England and North Wales are also relatively high with an average elevation higher than 400 metres and the rest of England is the lowest. In addition, it can be observed that the size of most catchments (around 99\%) in England and Wales are less than $600 \mathrm{~km}^{2}$ while only the catchments located near the boundary between England and Scotland have a larger size which is greater than $1000 \mathrm{~km}^{2}$. The catchment sizes vary greatly because these catchments are actually sub-catchments of the same river basin. The orientation of these catchments is indicated by the major angle $\omega$ referring to the North direction. In other words, $\omega=0$ indicates 
the orientation of catchment is north-south direction; the positive $\omega$ (presented by reddish colour) indicates a north-east orientation while the negative angle (presented by bluish colour) is a north-west orientation; and $\omega$ equaling to $\pm 90^{\circ}$ means the orientation of the catchment is east-west direction. It can be observed that the catchments near the north coastlines tend to be northeast orientated while the ones near east coastlines are northwest orientated. Moreover, the catchments located at the boundary of Wales and England tend to be north-south orientated indicating a very light red or blue colour (i.e., $\omega$ closed to 0 ). Referring to the shape of catchments shown in Figure 3.15c, the shape of most catchments $(61 \%)$ is relative rounded or elliptical and their $s p$ is in the range of 1.0 and 2.0. $s p$ to the rest majority is less than 4.0 indicating an elongated shape.

The correlation among these spatial features does not necessarily imply a causal relationship; one should be, however, more interested in how the parameters co-vary with the catchment characteristics. To explore more about catchment characteristics, Figure 3.15e and Figure $3.15 \mathrm{f}$ are generated where the spread of shape index $s p$ of smaller regions is greater than that of larger regions; while the elongated shape has a much higher probability of being accompanied with a small size while the large size of catchment tends to have a relatively rounded shape in both England and Wales. The relationship between the catchment shape and orientation is insignificant while the catchments with an elongated shape tend to have a northwest or northeast orientation $\left(\omega\right.$ around $\left.\pm 50^{\circ}\right)$. 


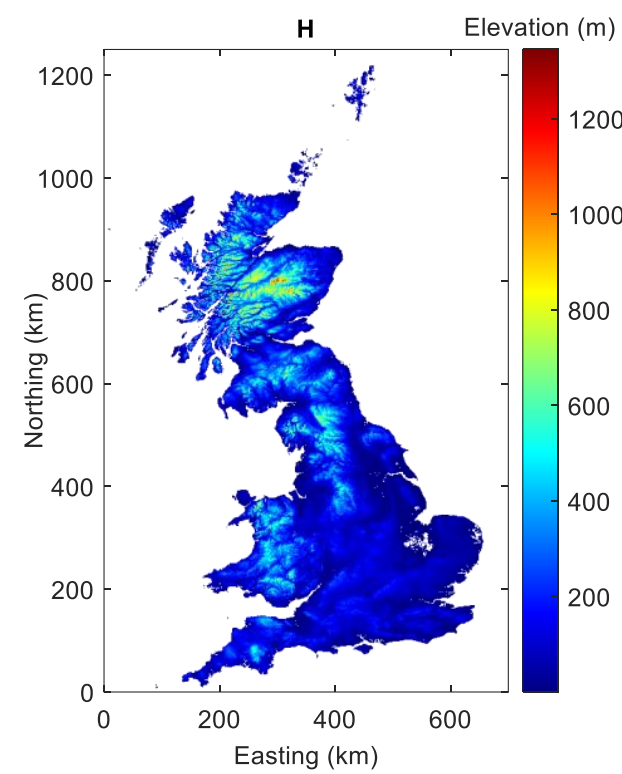

(a)

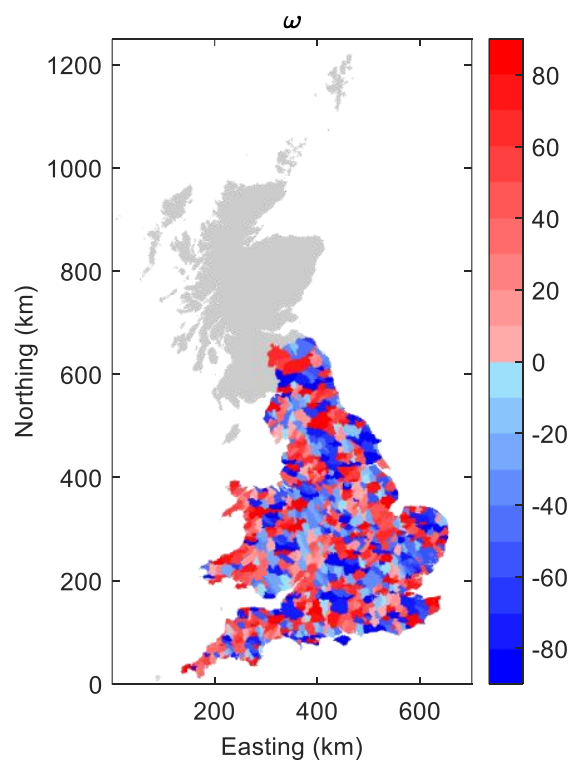

(c)

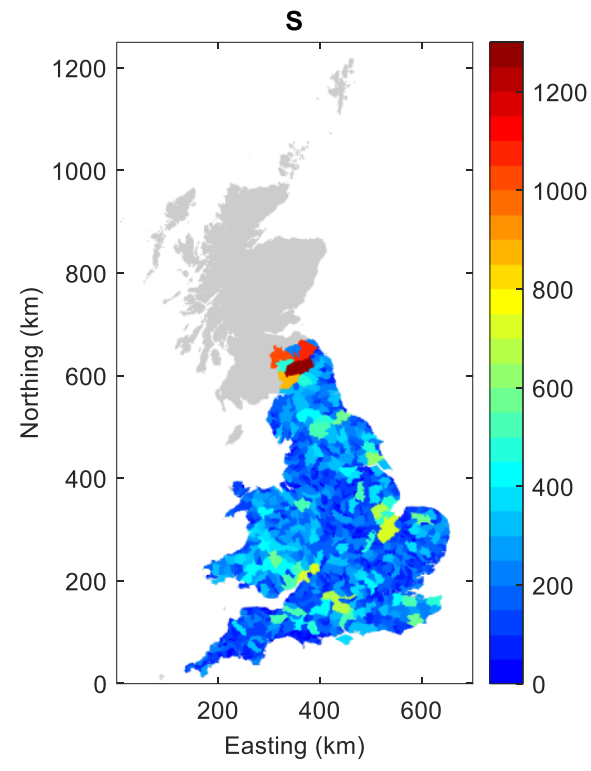

(b)

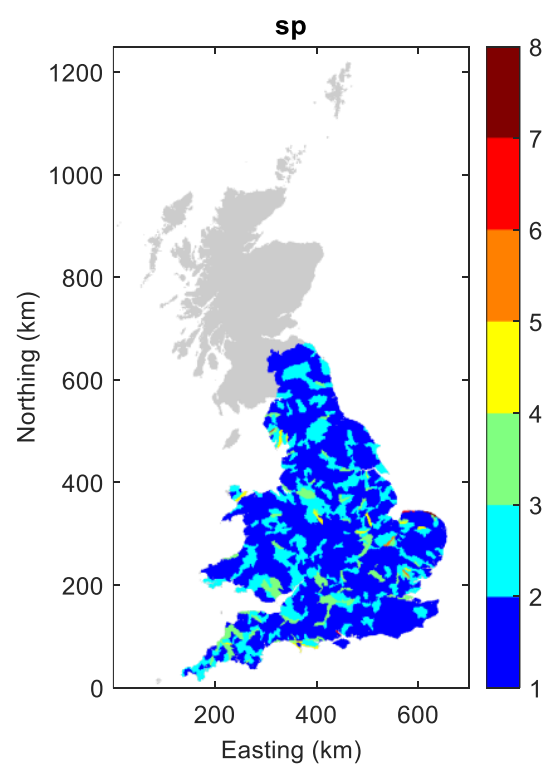

(d) 


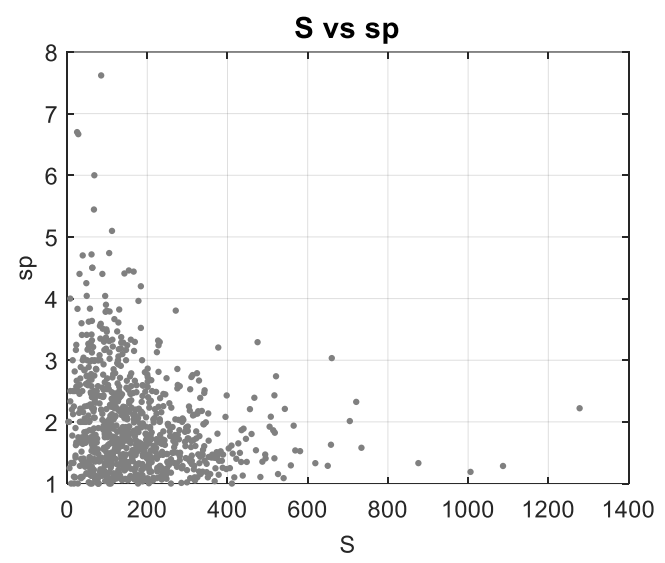

(e)

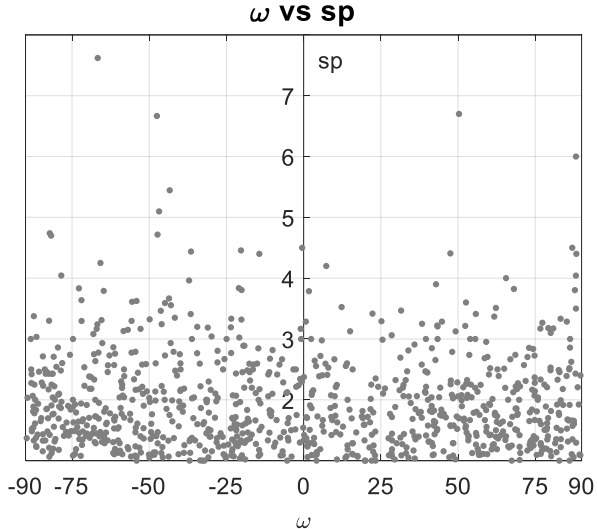

(f)

Figure 3.15 Spatial features of study catchments.

\subsubsection{Spatial variation of AMDR with respect to location and elevation}

Figure 3.16 presents the spatial patterns of the AMDR represented by three GEV parameters in the catchments over England and Wales (subfigures a, b and c), and how these parameters are related to the catchment elevation (def). Out of all catchments, there are around $80 \%$ follow the Fréchet distribution $(\xi>0$, shown as reddish colour in Figure 3.16a), mainly located in middle and eastern England of lower elevation; only 16\% follow the Weibull distribution $(\xi<$ 0 , shown as bluish colour), mainly located in the vicinity of Manchester and Liverpool and middle-western Wales where the elevation is relatively high (see Figure 3.15a); and the rest (4\%) following Gumbel distribution $(\xi=0)$.

Subfigures $\mathrm{b}$ and $\mathrm{c}$ of Figure 3.16 present the spatial variation of the GEV parameters $\sigma$ and $\mu$ by which the behaviour of AMDR can be parameterised and depicted. It can be observed that both $\sigma$ and $\mu$ present a similar spatial pattern where a higher $\mu$ is usually accompanied by a higher $\sigma$. Meanwhile, along the same Northing coordinate, the parameters of the western region, especially western Wales and Lake District of England, are much greater than the eastern area such as middle and east England. By contrast, the change of the parameters in the catchments located at the same Easting coordinate is not remarkable and the only difference that can be observed is that $\sigma$ and $\mu$ of the catchments in North England are higher than the South areas. Such spatial pattern can be described as "west high, east low" and the difference 
is not linearly either - there is a significant decrease occurring in the west while the gradient of such decrease is much smaller in the east. As $\mu$ can reflect the level of the most frequent AMDR in the last century and $\sigma$ can somehow tell the occurrence probability of those extreme events, the parameterization quantification can be translated as the most frequent AMDR in the west is usually higher than the east and those areas with a higher most frequent AMDR are usually observed to have a higher probability of the occurrence of extreme AMDR. These findings are the same as the result of the ROI-based study (see Section 3.4.1).

The corresponding Figure 3.16e and Figure 3.16f are presented to show the general relationship between the GEV parameters and the catchment elevation $H$ which is demonstrated by trend lines. In general, both parameters have a positive trend that catchments with a higher elevation have a higher level of most frequent AMDR as well as a higher occurrence probability of extremes. A plausible explanation is that the local topography can play an important role in enhancing extreme rainfall via processes such as the uplifting of moist air.

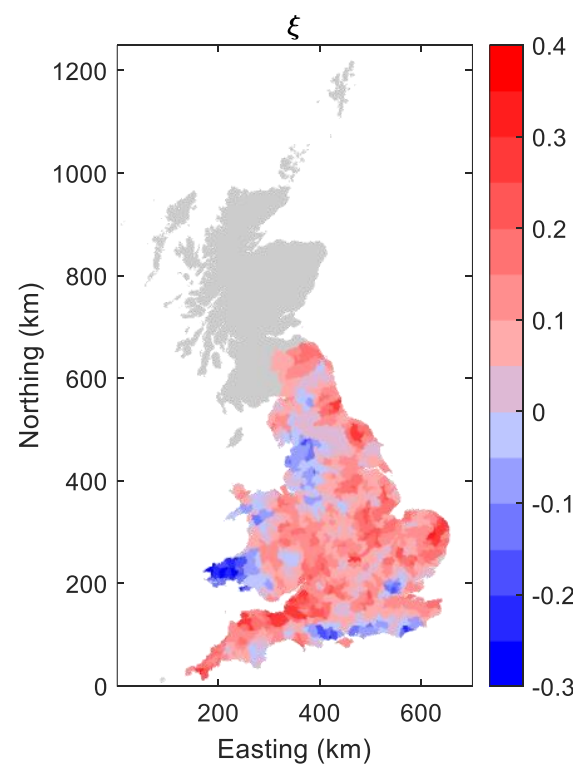

(a)

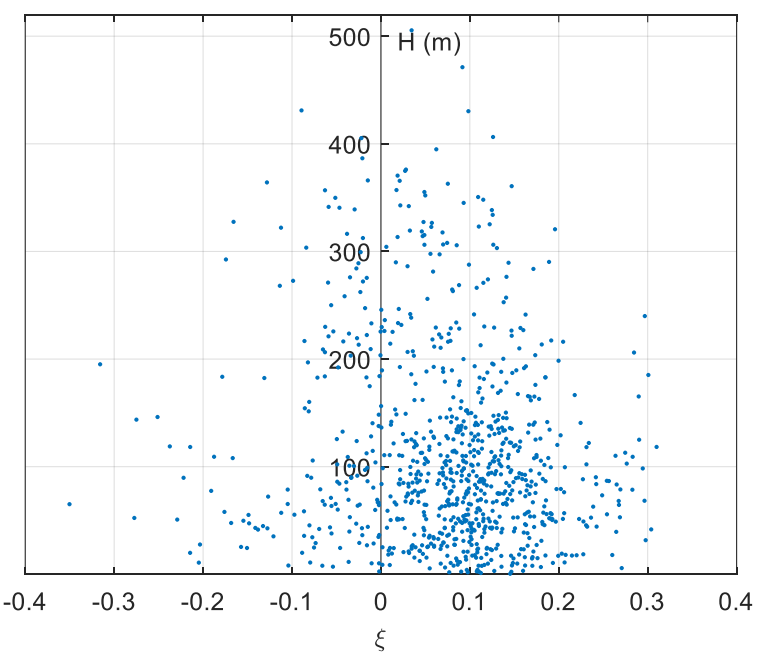

(d) 


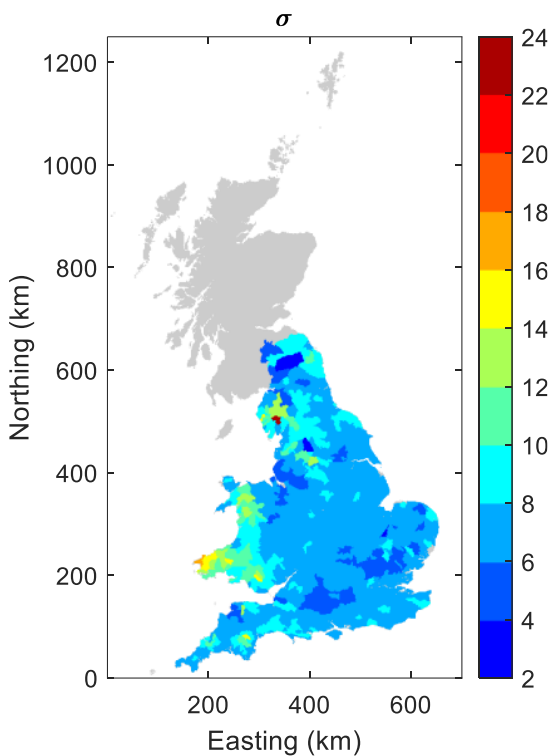

(b)

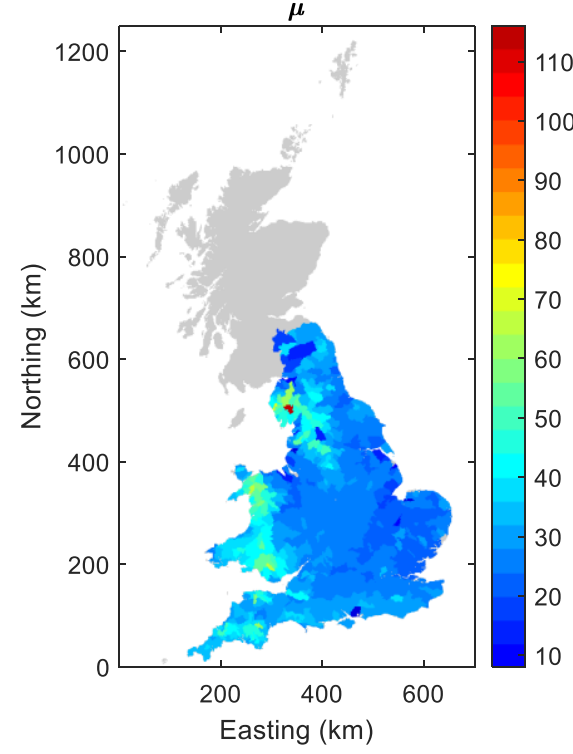

(c)

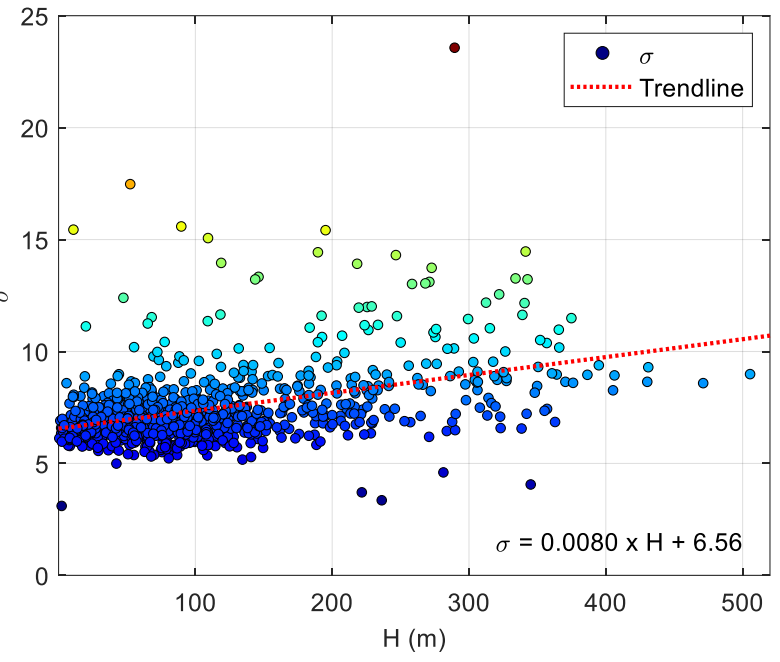

(e)

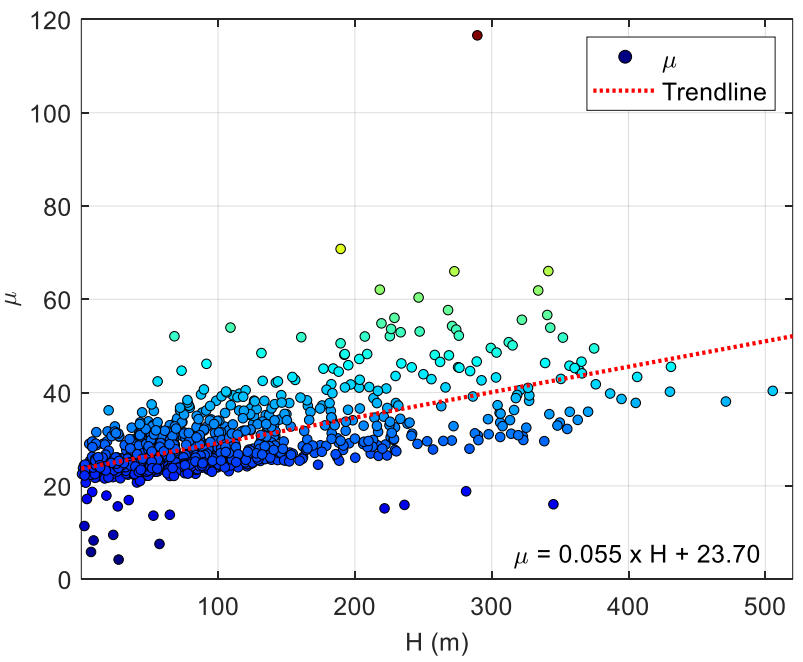

(f)

Figure 3.16 Spatial variation of AMDR with respect to catchment location represented by GEV parameters $\xi$

(a), $\sigma$ (b) and $\mu$ (c); and the relation between these three parameters and the catchment elevation $(H)$ is presented in d,e,f respectively.

\subsubsection{Spatial variation of AMDR with respect to catchment size}

The UK Meteorological Office usually classifies rainfall into four categories according to the rate of precipitation (Jebson, 2007): "slight" ( $0 \sim 2 \mathrm{~mm} /$ hour or roughly $0 \sim 5 \mathrm{~mm} /$ day), 
"moderate" (2 10 mm/hour or 5 25 mm/day), "heavy" (10 50 mm/hour or 25 125 $\mathrm{mm} /$ day), and "violent" ( $>50 \mathrm{~mm} /$ hour or $125 \mathrm{~mm} /$ day). To be more specific, in this study, I added several classes and divided the catchment-based AMDR at the most frequent level (w.r.t , $\mu$ ) into 5 groups, i.e., slight ( $<10 \mathrm{~mm} /$ day, $1.0 \%$ of all catchments), moderate $(10 \sim 25$ $\mathrm{mm} /$ day, $25.6 \%$ ), high (25 50 mm/day, 70.3\%), extreme (50 100 mm/day, 3.0\%), very extreme ( $>100 \mathrm{~mm} /$ day, $0.1 \%)$. The location of catchments belonging to these five groups as well as the GEV parameters $\sigma$ and $\mu$ changing over catchment size are shown in Figure 3.17, respectively. Catchments with moderate AMDR levels are mainly located in east England while those at the extreme level are located in the Lake District and North and West Wales near the coast.
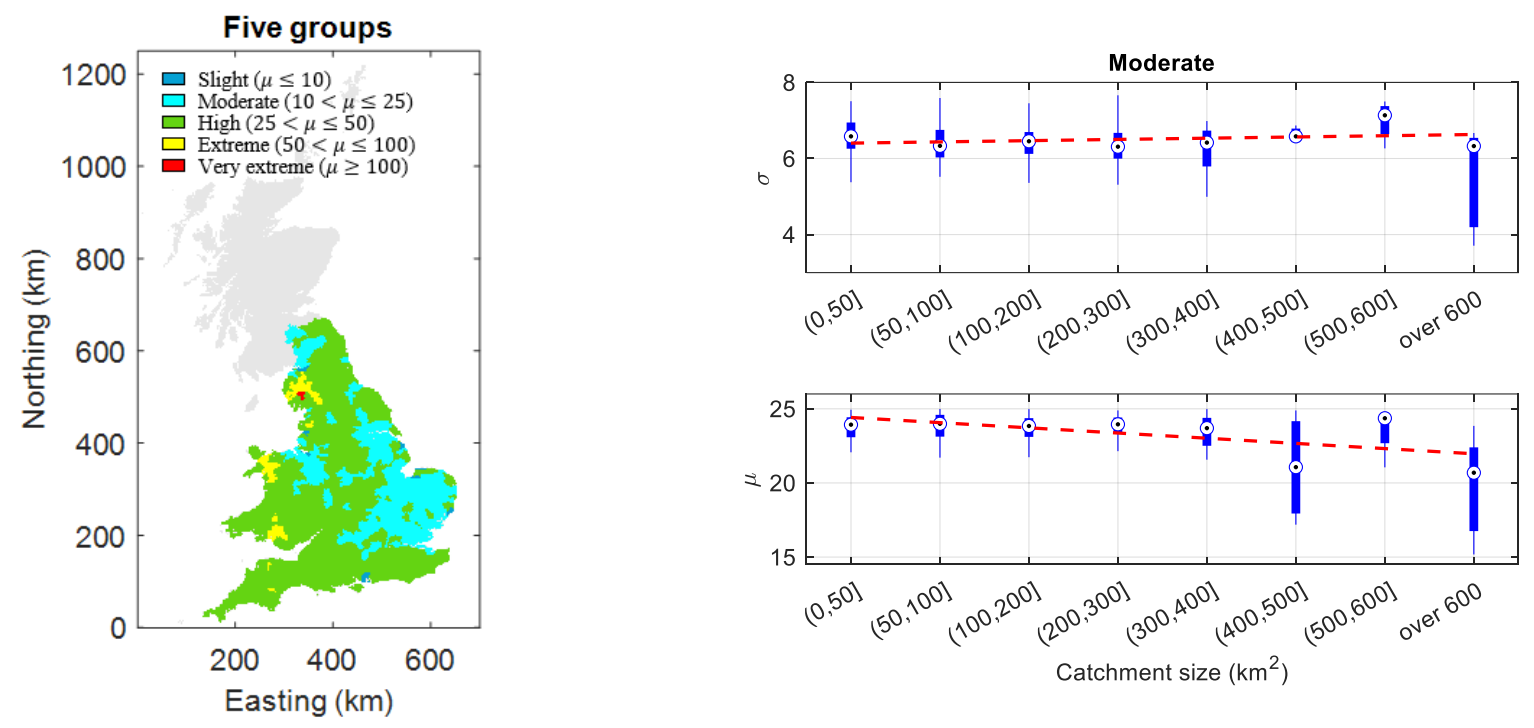

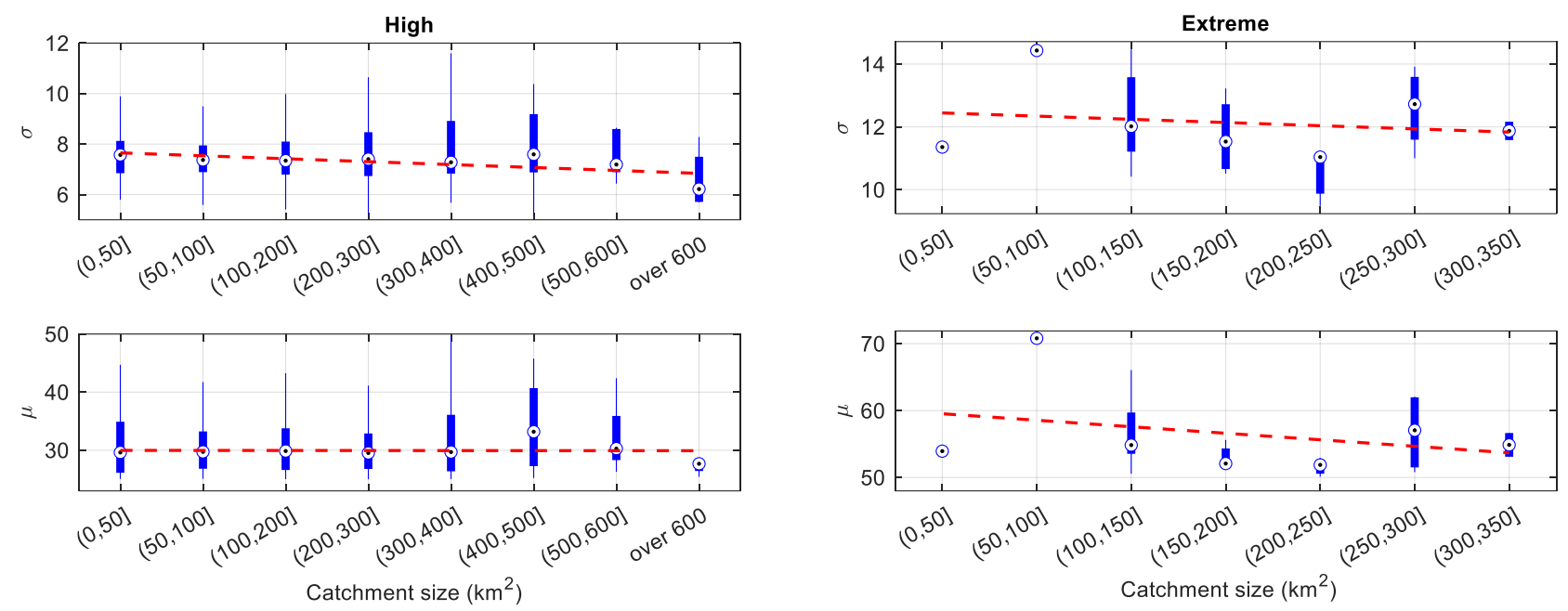

Figure 3.17 Categories of five groups according to the level of AMDR and GEV parameters $\sigma$ and $\mu$ changing with catchment size in the catchments with "Moderate", "High" and "Extreme" levels of AMDR.

In general, with the increase of catchment size, both parameters show a decreasing trend especially in the south and east England ("Moderate" group), middle-west Wales and the Lake District ("Extreme" group), which is caused by areal averaging and the same with the ROIbased study. However, for the "High" group with a middle level of the most frequent AMDR, a decreasing trend can be observed when the catchment size is either relatively small (e.g., less than $200 \mathrm{~km}^{2}$ ) or relatively large (e.g., greater than $500 \mathrm{~km}^{2}$ ). However, there is an increasing trend shown in the catchments of medium sizes $\left(400 \sim 500 \mathrm{~km}^{2}\right)$. This phenomenon shows that the change of parameters over catchment sizes is strongly affected by their geographic locations. For example, for the catchments in this group (those mainly located near the boundary of the high and extreme AMDR areas; see the catchments with the size of $400 \sim 500$ $\mathrm{km}^{2}$ in Wales and southern England in Figure 3.15b), if increasing the catchment size involves more grids with higher AMDR, the corresponding parameters $\mu$ and $\sigma$ will also increase because the reduction caused by areal averaging cannot be compensated by the involvement of more heterogeneous grids of higher rainfall. Such effect can go the other way as well when more grids of lower AMDR are included. This can explain the result for the "Extreme" group where an increasing trend is observed when the size is in the range of $200 \sim 300 \mathrm{~km}^{2}$. For the small catchments located in the Lake District and middle-west Wales where the AMDR are 
extreme, larger catchments tend to include more grids with higher rainfall thereby increasing $\mu$ and $\sigma$.

\subsubsection{Spatial variation of AMDR with respect to catchment orientation and} shape

Compared with the location, elevation and size of the catchments, the effect of catchment orientation and shape is not that significant; however, in order to demonstrate such relation more clearly, I fit the result using local linear regression (LLR; Baíllo and Grané, 2009; Fan, 1993 ) as the background of subfigures in Figure 3.18 to help the analysis. One of the most commonly used methods for carrying out LLR is Locally Weighted Scatterplot Smoothing (LOWESS; Cleveland, 1979; Moran, 1984) which can generate a smooth curve or surface to help figure out the relationship or trend between two GEV parameters and catchment features, i.e., orientation and shape in this case.

For the catchments in "Moderate" group, there is a small difference between the westnorthwest oriented and east-northeast oriented catchments on $\sigma$ and $\mu$ which tend to be smaller than that of the north orientation. However, both $\sigma$ and $\mu$ tend to decrease when the shape becomes more and more elongated. For those in "High" group, the majority of the catchments show small differences on both parameters between the west-northwest and east-northeast orientation while the $\mu$ parameters for the catchments with north-northeast orientation are usually higher than those of the catchments with north-northwest orientation. Generally, two parameters decrease with an increased $s p$ but smaller-sized catchments witness a converse trend. For "Extreme" group, the pattern of parameters changing over orientation is distributed symmetrically with $\omega=0$ (North) where catchments with a west-northwest orientation usually have higher parameters than east-northeast orientation. On the whole, several patterns can be summarised as:

1) In middle-west Wales and the Lake District of England where AMDR is high, both levels of the most frequent AMDR and occurrence probability of extremes are higher in the catchment orientated in a west-northwest direction than others. 
2) For the rest area of Wales and England, generally, the level of the most frequent AMDR and occurrence probability of extremes in the catchments whose orientations are westnorthwest or east-northeast are almost the same and lower than that the north-south oriented catchments.

3) The level of the most frequent AMDR and occurrence probability of extremes in the catchments with an elongated shape are usually lower than others with a relatively rounded shape.

4) Catchment orientation and shape are not as much significant as their locations and sizes concerning the spatial effect on AMDR.
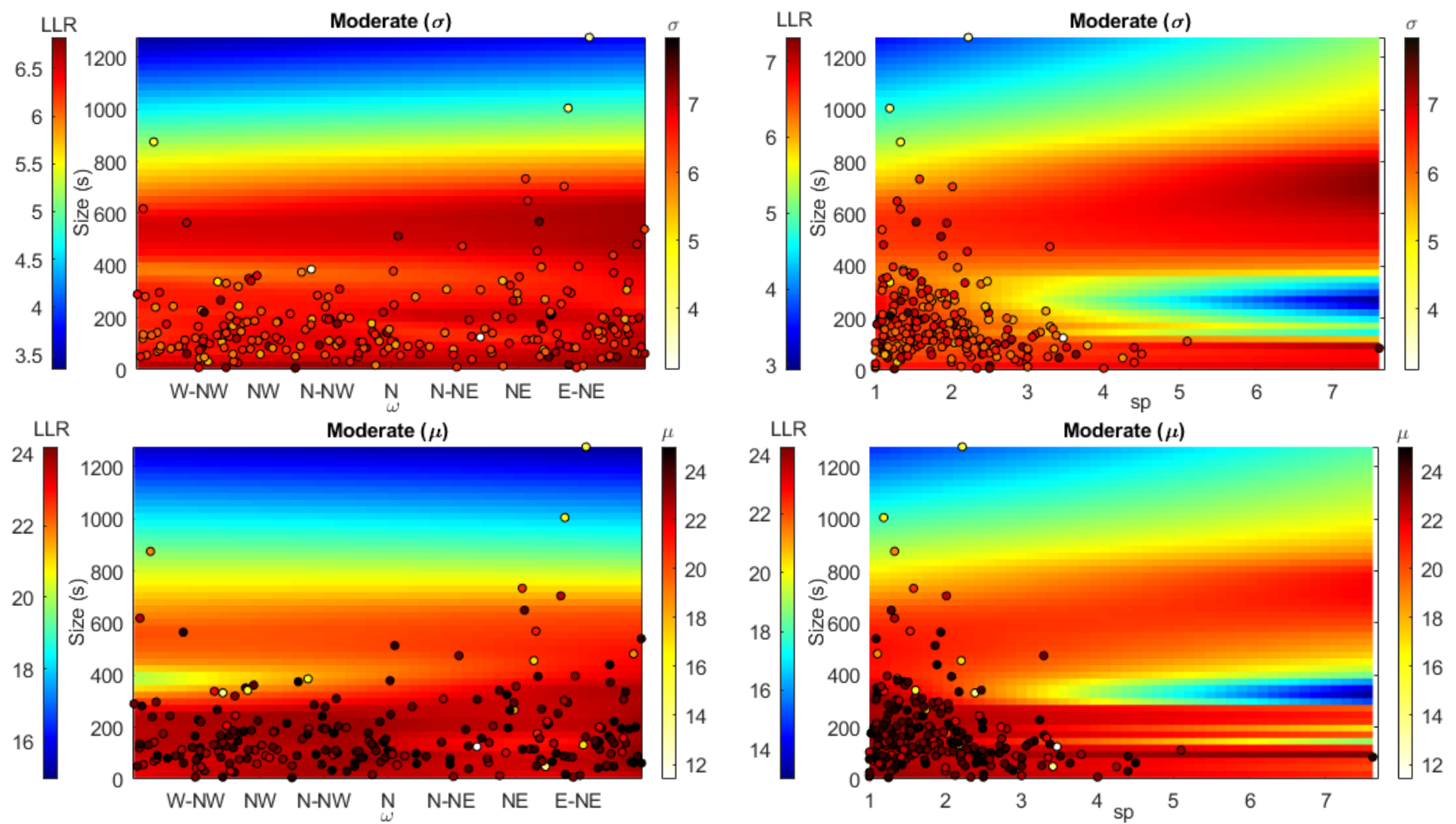

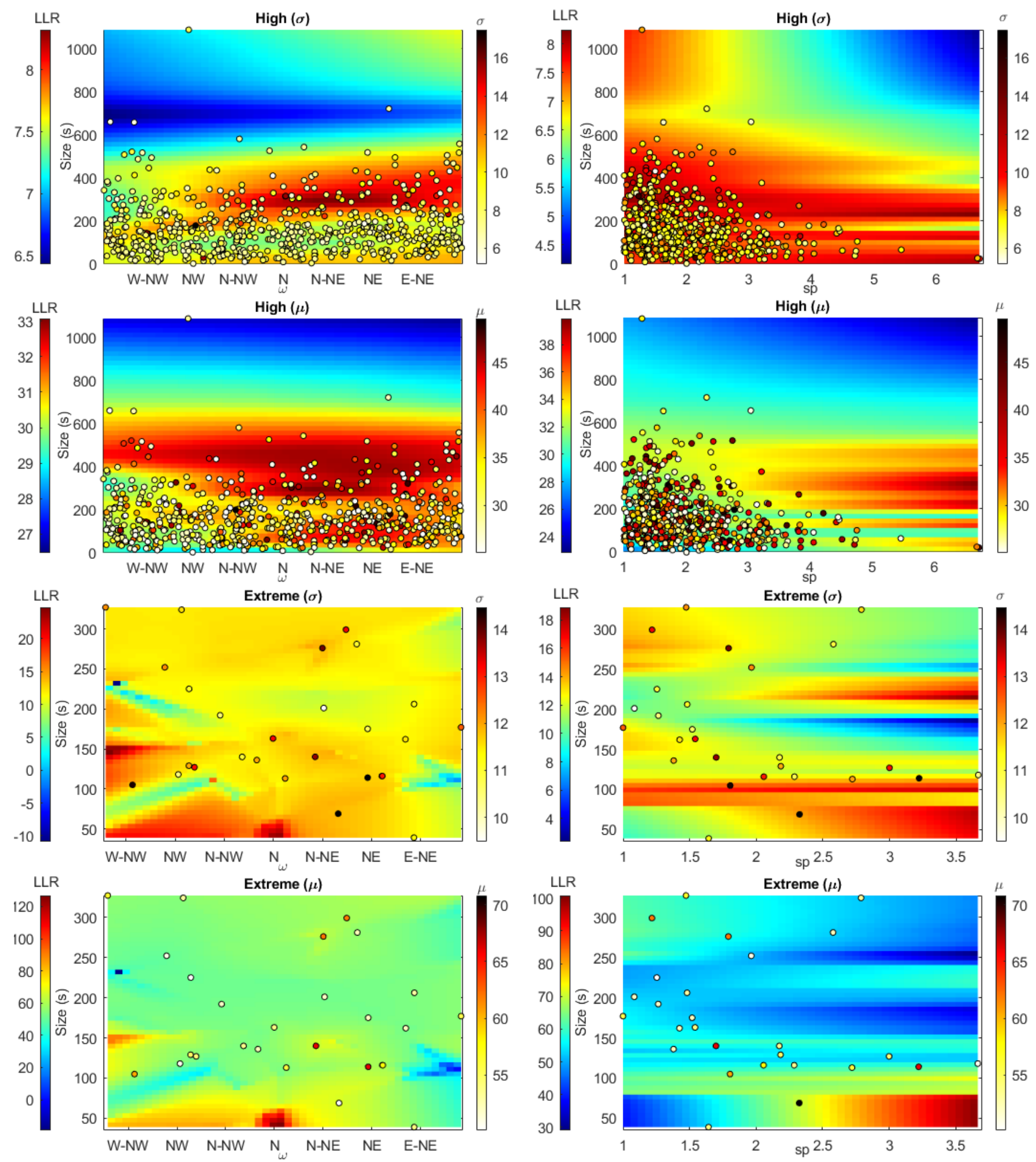

(a)

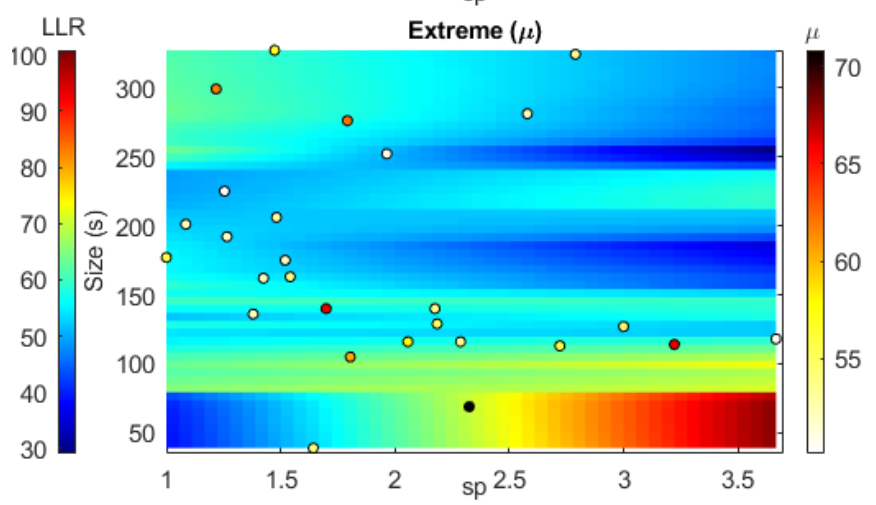

(b) 

climatology of rainfall variation

Figure 3.18 GEV parameters $\sigma$ and $\mu$ change over orientation $\omega$ (a) and shape $s p$ (b) of catchments in the three groups where "LLR" is short for "local linear regression" and the directions "North", "West" and "East" are abbreviated as "N", "W" and "E”.

\subsection{The link between the spatial variations of GEV parameters and the large-scale climatology of rainfall variation}

The GEV distribution parameters that can reveal the characteristics of extreme rainfall in terms of both its amount and occurrence probability, are shown to have a strong spatial dependency as discussed previously. To understand how such spatial variation of the extreme rainfall is related to the climatology of rainfall variation, the areal annual rainfall (AAR) time series from each ROI was obtained. The mean and standard deviation (SD) of the AAR series were then compared with the GEV parameters $\mu$ and $\sigma$ of the AMDR series extracted from the same ROIs. To visualize the link, the spatial continuity of the corresponding parameters from both AAR and AMDR was represented by their variograms (see Figure 3.19) which shows very little difference in locative continuity.

For the GB case, when the distance between the adjacent ROIs is less than around $300 \mathrm{~km}$, both $\mu$ and the mean of areal annual rainfall (AAR) are spatially related. However, there is no sill observed in $\sigma$ and the standard deviation of AAR. For AU, the ranges for spatial related ROIs for $\mu$ and $\sigma$ of AMDR are around $300 \mathrm{~km}$, which is similar to those for the mean and standard deviation of AAR. 


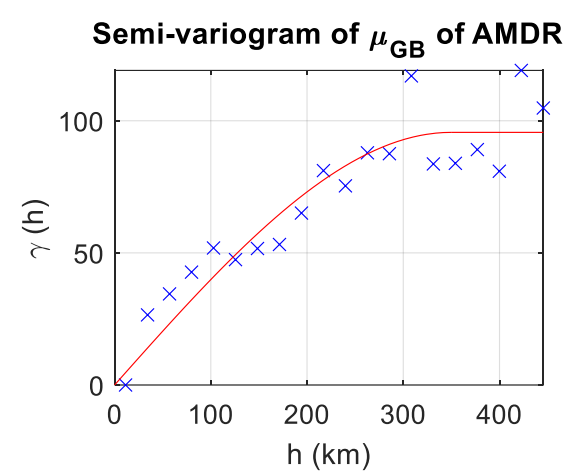

Semi-variogram of mean of AAR (GB)

Semi-variogram of $\sigma_{\mathrm{GB}}$ of AMDR
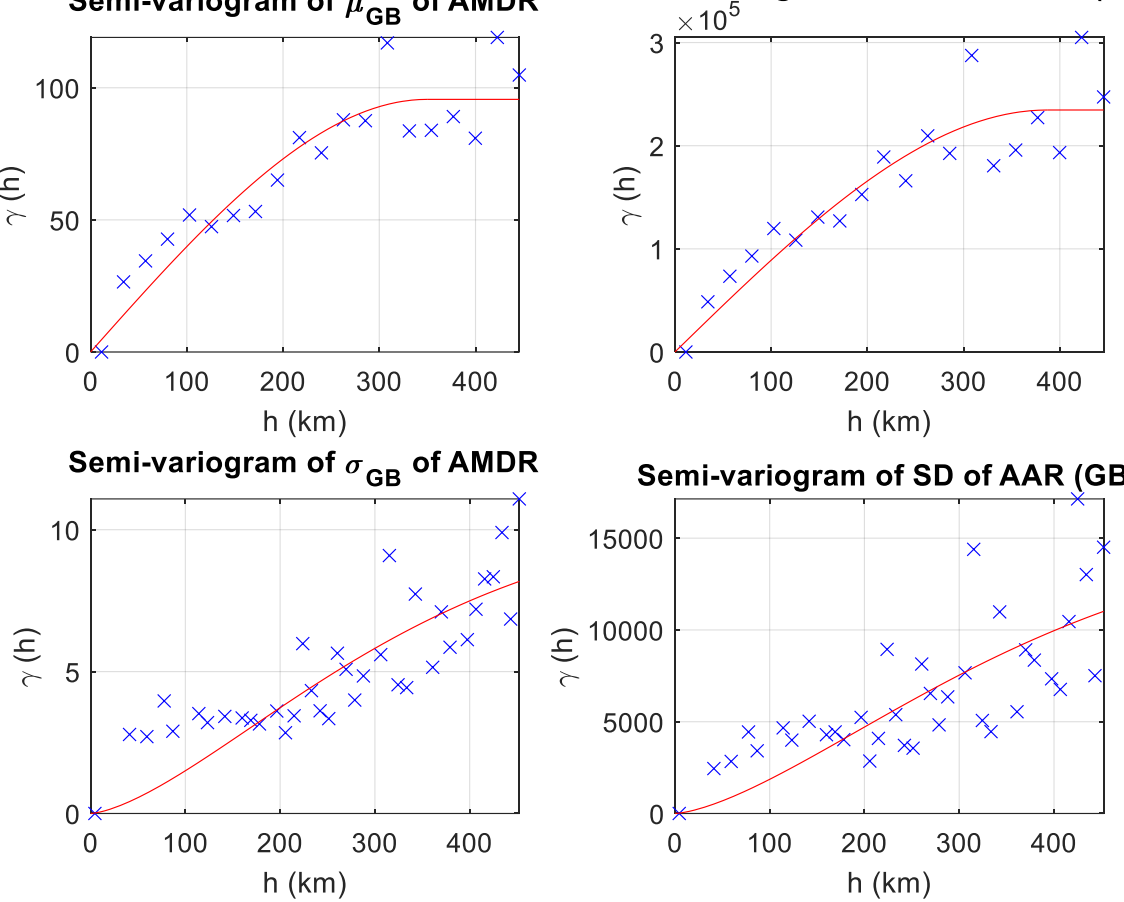

(a)

Semi-variogram of $\mu_{A U}$ of AMDR

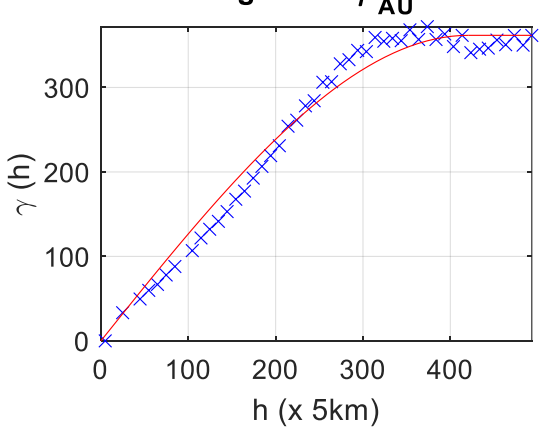

Semi-variogram of mean of AAR (AU)

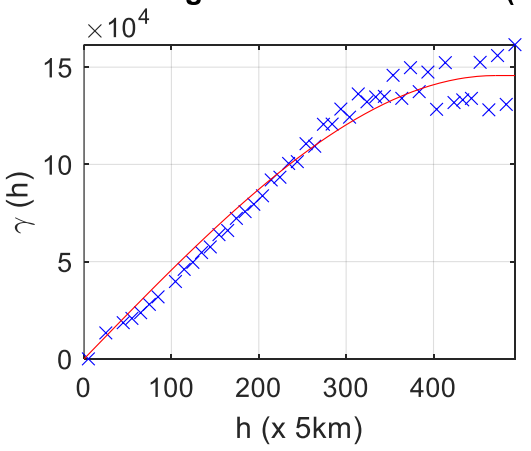

Semi-variogram of $\sigma_{\text {AU }}$ of AMDR
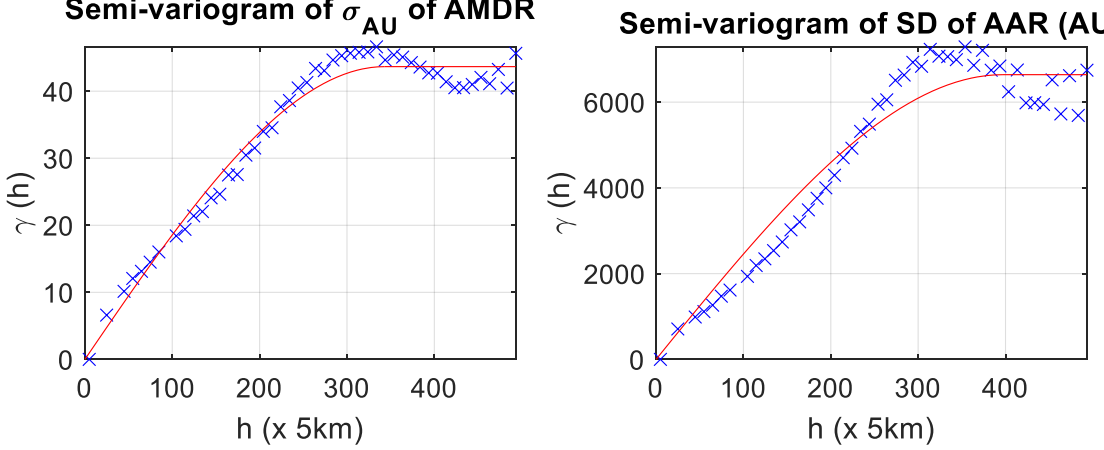

(b) 

climatology of rainfall variation

Figure 3.19 The variograms for comparing spatial continuity of GEV parameters $\mu$ and $\sigma$ with spatial continuity of areal annual rainfall (AAR) 's mean and their standard deviation (SD) of GB (a) and AU (b) cases fitted by Spherical model.

Figure 3.20 demonstrate a great deal of similarity exiting in space between the daily maxima time series (i.e., AMDR) and the cumulative annual rainfall (i.e., AAR). For example, regions with higher mean of AAR are not only represented by higher SD (e.g., the circles located in west Scotland and west Wales of GB and in north-eastern coastal regions of AU, appearing more reddish and larger), they are also associated with higher GEV parameters of AMDR, and appear to be more heterogeneous. This feature also exists in the regions with low and more even annual rainfall distribution, but works in an opposite way (e.g., circles located in middle and eastern England of GB and middle-north zone of AU are all more bluish and smaller). These findings are consistent with those published in the series of climate reports of both countries.

In GB, a series of annual state of climate reports (Kendon et al., 2015; Kendon et al., 2018, 2019) released in the past few decades by Met Office show that:

1) Rainfall in eastern England has a much more even distribution while rainfall of midland at the same latitude is higher than eastern England but the wettest month varies more across this region;

2) Wales varies most widely with the highest average rainfalls;

3) Scotland has the most remarkable increase in average rainfall and altitude is the greatest effect factor of rainfall distribution.

These phenomena can be explained and also revealed by the spatial distribution of two GEV parameters in the analysis of Figure 3.20: 1) the amount of most frequent rainfall (w.r.t. $\mu$ ) in western GB are higher than the east, especially in the Highland of Scotland and Wales; 2) the extremes are more likely to be accompanied by a high amount of most frequent rainfall. The 

climatology of rainfall variation

extreme rainfall in Wales and west Scotland varies most compared with eastern GB where the rainfall is more evenly distributed (w.r.t. $\sigma$ ).

In $\mathrm{AU}$, the climate also has a strong spatial dependency and is changing in response to a warming global climate system with a change of both frequency and severity of extreme weathers as shown by annual climate reports produced by CSIRO and the Australian Bureau of Meteorology (CSIRO and Meteorology, 2018) which state that:

"There has been a significant drying across southern AU and last century with lower-thanaverage growing season rainfall which is expected to be more frequent than in the past. An increasing proportion of Australia received more rain from heavy rain days during the period 1950-2012 and large variability in extreme rainfall events from decade to decade is also evident, with very wet events often associated with La Niña years."

This feature is clearly reflected in the GEV parameters in the southern AU where $\mu$ indicating the amount of most frequent rainfall is low (less than $20 \mathrm{~mm}$, dry climate) but $\sigma$, although is not high when being compared with the coastal regions, varies across this area (from less than 10 to 15 ) and the relatively high $\sigma$ value shows a relatively high occurrence probability of extremes such as extreme events of lower-than-average rainfall. However, the situation is different in north-eastern coastal areas of AU with a high $\mu$ and a varying $\sigma$. This finding is also consistent with the explanation in the cited climate report. 

climatology of rainfall variation

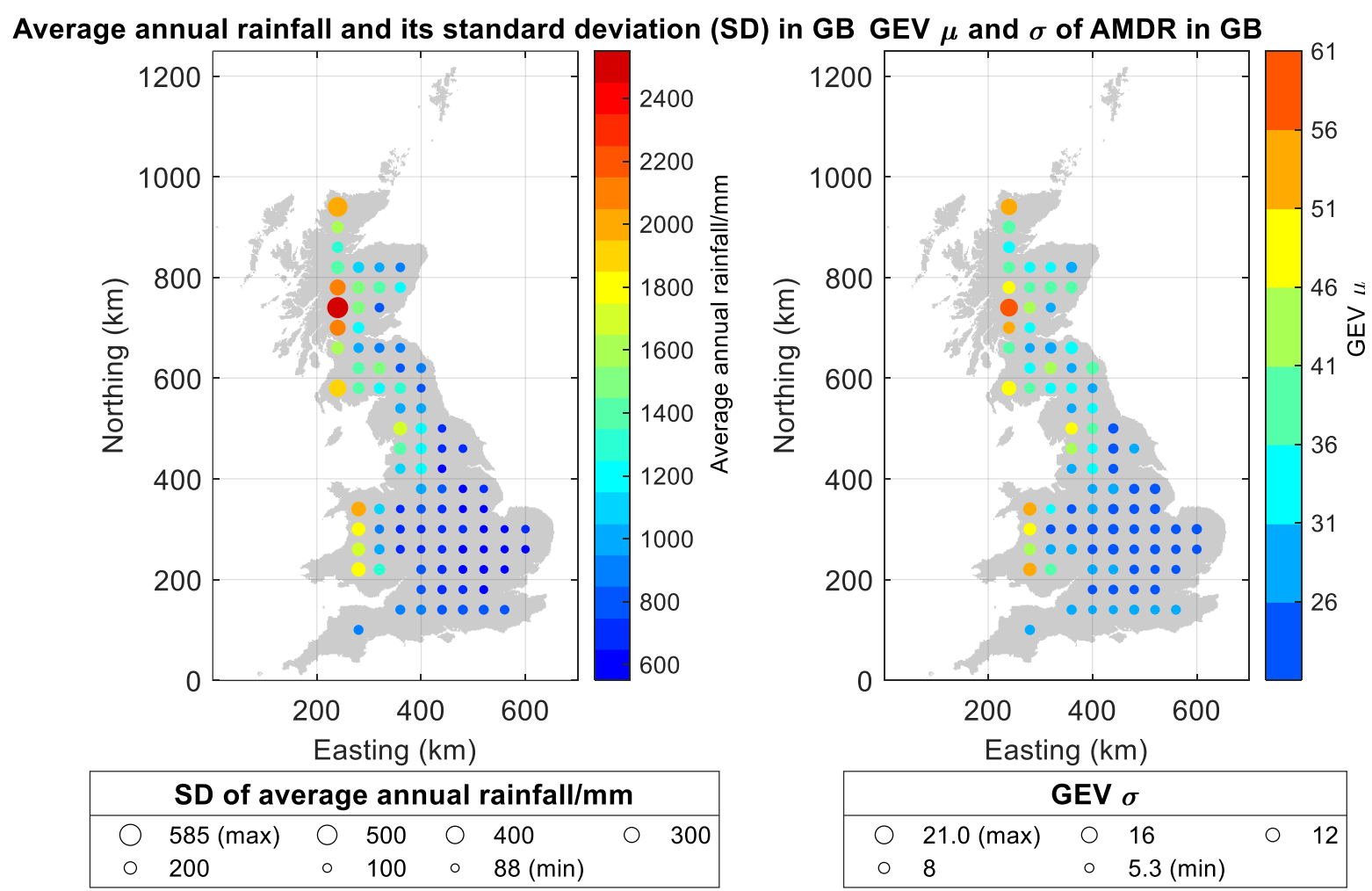

(a) 

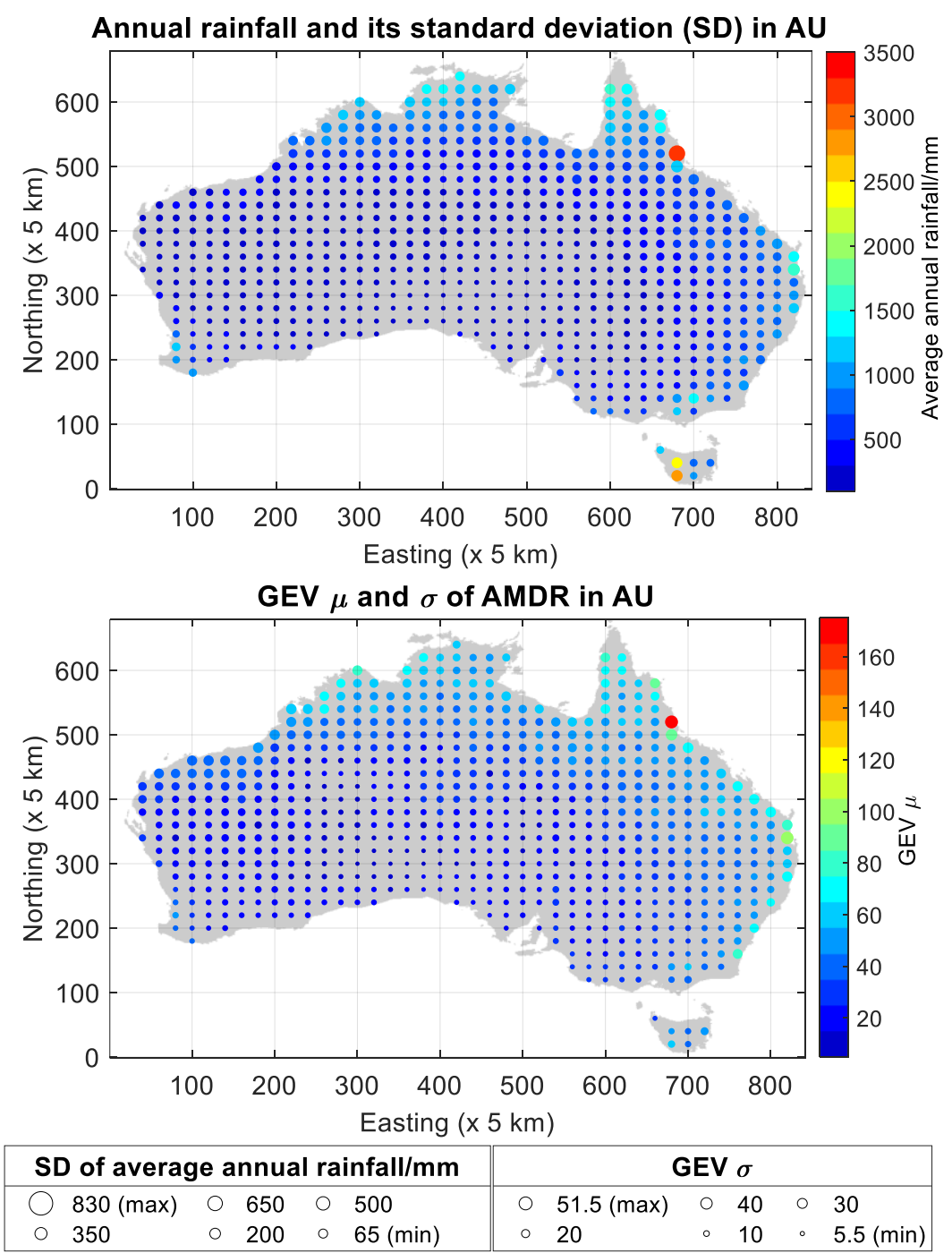

(b)

Figure 3.20 Comparison between the climatic variables (the average annual rainfall and its standard deviation) and GEV parameters $\mu$ and $\sigma$ in GB (a) and AU (b) cases where the colour denotes the value of averaged annual rainfall or the GEV parameter $\mu$, and the size of the circles denotes the value of the standard deviation of the annual rainfall or the GEV parameter $\sigma$.

\subsection{Summary and remarks}

This chapter presents a quantitative study of the spatial variation of extreme rainfall with regards to various spatial characteristics such as location, size and shape/orientation, using two- 
century long datasets covering Great Britain (GB) and Australia (AU). First, an ROI-based approach is developed where a large number of regions of interest (ROIs, 11,011 in total) are randomised by altering their spatial properties using the SRS-GDA toolbox (presented in Chapter 2). The annual maximum daily rainfall (AMDR) series extracted from these ROIs are individually fitted with a well-tested GEV distribution whose parameters are then analysed over space. The relationship between the rainfall extremes and the various spatial properties of the ROIs is validated by a catchment-based analysis where 903 real catchments in England and Wales are involved and further quantified by developing four generalised linear models (GLMs). As the result from the real catchments shows good consistency with the ROI-based study, the accuracy and effectiveness of the proposed ROI-based approach specifically designed for large grid-based datasets are well-validated and the latter can be readily applied to other hydro-climatic quantification analysis for evaluating the spatial heterogeneity of climate change impacts, such as flooding and droughts.

From the results discussed previously based on the GB and AU application cases, the following conclusions can be drawn:

1) The GEV distributions are shown to be able to model well the grid-based areal AMDR for both the GB and AU cases; more than $90 \%$ of ROIs and $80 \%$ of real catchments are better fitted with the Frechét type of distribution among the three GEV types.

2) Most catchments (around 99\%) are less than $600 \mathrm{~km}^{2}$ while only the boundary of England and Scotland have a larger size greater than $1000 \mathrm{~km}^{2}$; The catchments near the north coastlines tend to be northeast orientated while the ones near the east coastlines are northwest orientated and the catchments at the boundary of Wales and England tend to be north-south orientated. And the shape of more than half catchments $(61 \%)$ is relative rounded or elliptical.

3) The deviation of the shape of smaller regions is greater than that of larger regions and the elongated shape has a much higher probability to be observed in small-sized catchments however the large catchment tends to have a relatively rounded shape in England and Wales. 
4) The GEV location $(\mu)$ and scale $(\sigma)$ parameters present similar spatial patterns where a higher $\mu$ is usually accompanied by a higher $\sigma$ indicating those regions that have a higher amount of most frequent rainfall often observe a higher occurrence probability of extremes. And catchments with higher elevation usually get a higher level of most frequent AMDR and occurrence probability of extremes.

5) Geographic location is the most significant factor affecting the two GEV parameters. The spatial pattern in GB is an eastward decreasing banded pattern with no significant difference along the north-south direction. In AU, a concentrically increasing pattern from the middle-south zone to northeast coasts is found.

6) Increasing the region size will decrease both parameters which means a decrease of the most frequent AMDR amount and the occurrence probability of extremes. However, in $\mathrm{AU}$, the rate of such decrease varies with regions as the combined impact of ROI location and size is also detected to be significant. In the catchment-based study, generally with the increase of catchment size, both parameters show a similar decreased trend caused by the areal average especially in the south and east England (where AMDR is relatively low), Lake District and middle-west Wales (where AMDR is very high). However, for the catchments with the middle level of AMDR, a decreased trend can be observed when the catchment size is either very small or very large but in between, both parameters increase. This phenomenon shows that the change of parameters over catchment sizes is affected by their geographic locations as well when increasing size by involving more grids of higher rainfall can overcompensate the reduction caused by the areal average.

7) Compared with other spatial properties, the shape of ROI is detected as insignificant, even though, a symmetric pattern is found for regions with reciprocal spatial indexes. Also, regions of more elongated shapes tend to have small parameter values in contrast with those having regular/rounded shapes. However, in middle-west Wales and Lake District of England where AMDR is high, both parameters are higher in the westnorthwest-oriented catchment than in other orientations. And for the rest area, parameters in the catchments whose orientations are west-northwest or east-northeast are almost the same and lower than the north-south orientation. 
These findings offer new quantitative insight in understanding the spatial variation of largescale climatology of rainfall. Not only are they supported and consistent with many previous studies on rainfall distributions, but the quantification of the extreme rainfall and its spatial dependencies are also of great practical value in engineering design, e.g., designed rainfall/floods for constructions. 


\section{Chapter 4 Modelling Temporal Variation of Rainfall Extremes}

Continuing from the studies presented in the previous two chapters, this chapter focuses on the temporal quantification of rainfall extremes in parallel with the spatial variation models depicted in Chapter 3, aiming to address (part of) Q2 and Q4. The study discussed in this chapter ${ }^{5}$ demonstrates the process of modelling the extreme rainfall using both stationary and nonstationary Generalised Extreme Value (GEV) models over a large number of ROIs distributed over GB and AU for the last century, aiming to gain insights into the spatial variation of the GEV distribution in modelling extreme rainfall. Alongside the L-Moments (LM) and Maximum Likelihood (ML) estimation methods, the Bayesian Markov-Chain Monte Carlo (B-MCMC) method is employed to estimate the parameters in the nonstationary condition. The results show that a large proportion of the ROIs in both countries can be best modelled by nonstationary GEV models as far as the annual maximum daily rainfall (AMDR) is concerned. The most frequent AMDR, represented by the location parameter of the GEV, tends to be increasing over time especially in the coastal regions of GB and western Australia. Increasing the region area will decrease the baseline values of the GEV location and scale parameters and the time-varying terms due to climate change in most situations. However, in certain locations, increasing the area can amplify the climate change impact. Region shape is the least significant factor compared with the other two spatial features, but a symmetric pattern is observed. Furthermore, the comparison between different models shows that the conventionally used stationary models can underestimate remarkably the AMDR in regions

\footnotetext{
${ }^{5}$ Part of the contents of Chapter 4 has been submitted to the journal "Weather and Climate Extremes" and under review.
} 
where the nonstationary model is preferred. The findings suggest that an overhaul of the current storm design determination procedure may be needed in view of the impact from not only the environmental change but also the spatial variation in natural processes.

\subsection{Introduction}

As discussed in the literature review in Section 1.2.1, recently, there has been a growing interest in studying natural events from a climate-change perspective, given that key hydro-climatic variables, such as precipitation, temperature, streamflow, etc., are indeed changing due to the impact of climate change (Zscheischler et al., 2018). For the commonly used nonstationary GEV model, this is meant to assume that its scale and location parameters are varying with time or other climate indices (Son et al., 2017) and in the last few decades there have been several studies applying nonstationary GEV distributions to fit extreme rainfall. However, most of them focused on a limited number of specific domains because of data availability issues in hydrological observations; therefore, their conclusions are mostly of rationale and lack of generalization (Ganguli and Coulibaly, 2017). Meanwhile, it is clear that hydroclimatic extremes such as extreme rainfall can be affected by its local features not only the topography but also the orientation (shape) and size of the area, which is presented in Chapter 3. However, how the area-orientated rainfall extremes vary with the ROIs' geographical location, size and shape in the perspective of nonstationarity has not been fully studied; yet it is challenging as the variability of extremes can be sensitive to the size of the regions studied, e.g., substantial trends over smaller regions can arise purely from natural variability (Brown, 2018; de Leeuw et al., 2016; Fischer and Knutti, 2014).

This chapter presents a comparative study of Great Britain (GB) and Australia (AU) using two century-long grid-based (daily and 1-5 km) rainfall datasets, i.e., the GEAR dataset and the ADAM dataset (see Table 1.1 on Page 20 of Chapter 1), and it aims to gain the much-needed insights into the spatial variability of extreme rainfall associated with dramatically different 
climate and geomorphological features (GB and AU), as represented by the nonstationary probability distribution parameters. To achieve this, and not to be limited by the regional boundaries, I employed a sampling toolbox (Chapter 2) to generate a large number of ROIs by randomizing their locations, sizes and shapes, which is all presented in Table 3.1 on Page 50 of Chapter 3. For each ROI, the annual maximum daily rainfall (AMDR) time series were extracted with the assistance of high-performance computing (HPC) and fed in both stationary and nonstationary GEV models to address the impact of climate change on extreme rainfall. Finally, the patterns changing with three spatial features and the contrasting differences between stationary and nonstationary conditions at different return levels were analysed.

Specifically, the study attempts to address the research questions (the other part of) Q2 and Q4 and the main objectives include:

1. to reveal the extreme daily rainfall pattern that varies with time during the last century in two countries;

2. to assess the applicability of both stationary and nonstationary GEV models;

3. to test the three mainstream parameter estimation methods with regards to their goodness of fit at different levels of the rarity of rainfall extremes;

4. to evaluate the climate change impact in both countries and how it changes over time and space.

The remainders of this chapter start with the presentation of the main methodology including Block Bootstrapping Mann-Kendall (BBS-MK) test and parameter estimation for both stationary and nonstationary GEV models in Section 4.2; then it shows the results alongside a detailed discussion focusing on the spatial feature of the stationary and nonstationary GEV models (Section 4.3.2); spatial changes with ROI sizes (Section 4.3.3) and shapes (Section 4.3.4). The comparison at different return levels is discussed in Section 4.4. The conclusions and recommendations are given in Section 4.5. 


\subsection{Methodology}

The following approach is applied and covers the three related aspects of this study:

- Generate ROIs with varying location, size and shape and extract the maximum time series with the assistance of high-performance computing (HPC).

- Fit the time series obtained at every ROI with stationary and nonstationary GEV models with different parameter estimation methods.

- Evaluate the performance of all models and analyse the changes of time-varying parameters with regards to the geographical locations, sizes, and shapes as well as the level of extremity.

\subsubsection{Block bootstrapping Mann-Kendall (BBS-MK) test}

The Mann-Kendall (M-K) test (Kendall, 1948; Mann, 1945) is a nonparametric method to detect the monotonic trends in a series of hydrometeorological data, which is recommended by the World Meteorological Organization and has been widely applied in the hydroclimatic research area (Fathian et al., 2016; Song et al., 2014; Yue et al., 2002). The null hypothesis $H_{0}$ of the test is that the data (i.e., the time series AMDR in this study) come from a population that is independent, identically distributed; and the alternative hypothesis $H_{A}$ is that the data have a monotonic trend. Therefore, for the time series AMDR $X=\left(x_{1}, x_{2} \ldots x_{n}\right)$, the M-K test statistic $\mathrm{S}$ is given by:

$$
S=\sum_{k=1}^{n-1} \sum_{j=k+1}^{n} \operatorname{sgn}\left(x_{j}-x_{k}\right)
$$

With

$$
\operatorname{sgn}(x)=\left\{\begin{array}{c}
1 \text { if } x>0 \\
0 \text { if } x=0 \\
-1 \text { if } x<0
\end{array}\right.
$$

where $n$ is the length of AMDR which equals 113 for GB case and 129 for AU case. The mean of $S, \mathrm{E}(S)$ is 0 and the variance $\operatorname{Var}(S)$ can be calculated as: 


$$
\operatorname{Var}(S)=\frac{n(n-1)(2 n-5)-\sum_{j=1}^{p} t_{j}\left(t_{j}-1\right)\left(2 t_{j}+5\right)}{18}
$$

where $p$ is the number of the tied groups in the time series and $t_{j}$ is the number of data in the $j$ th tied group. The standardized normal test statistic $Z$ is employed for approximating the statistic $S$ is normally distributed:

$$
Z=\left\{\begin{array}{ccc}
\frac{S-1}{\sqrt{\operatorname{Var}(S)}} & \text { if } & S>0 \\
0 & \text { if } & S=0 \\
\frac{S+1}{\sqrt{\operatorname{Var}(S)}} & \text { if } & S<0
\end{array}\right.
$$

If the statistic $Z$ is greater than the critical value at 0.05 significant level in this study, the null hypothesis will be rejected. The positive or negative $Z$ indicates an increasing or decreasing trend. Besides, the magnitude of the trend can be evaluated by Sen's slope $\beta$ which is given by (Sen, 1968):

$$
\beta=\operatorname{Median}\left(\frac{x_{j}-x_{i}}{j-i}\right), j>i
$$

However, the basic assumption of the MK test is that data are serially independent. For example, if testing data have a positive autocorrelation, the occurrence possibility of the Type 1 error of rejecting the null hypothesis when it is correct is increased because of inflation of variance of M-K test statistic (Von Storch, 1999). Therefore, in the study, I employed the Block Bootstrapping Mann-Kendall (BBS-MK) test (Kundzewicz and Robson, 2004; Önöz and Bayazit, 2012; Sonali and Kumar, 2013), which is a robust and flexible approach for detecting the trend of AMDR. It firstly randomly resamples the AMDR in predetermined blocks without any modification of original data structure or autocorrelation and in bootstrapping, I shuffled 2000 times. Then the test statistic is calculated for each sample and its probability distribution is obtained. Finally, the test statistic from the resampled data is then compared with the test statistic from the original data to estimate the level of significance. Although there is a tradeoff between the Type I error and the power of the BBS-MK test, the results are not very sensitive to the selection of block length (Önöz and Bayazit, 2012). 


\subsubsection{Stationary generalised extreme value model (S-GEV)}

For a given ROI, The AMDR time series extracted at each ROI is then fitted by the GEV distribution whose cumulative distribution function (CDF) is defined as:

$$
F(x ; \sigma, \mu, \xi)=\exp \left[-\left(1+\xi\left(\frac{x-\mu}{\sigma}\right)\right)^{-1 / \xi}\right]
$$

The cumulative probability function $F$ is defined for $1+\xi(x-\mu) / \sigma>0,-\infty<\mu<\infty, \sigma>$ 0 and $-\infty<\xi<\infty$, where $\mu$ is the location parameter, $\sigma$ is the scale parameter, and $\xi$ is the shape parameter. There are three types of distribution in the GEV family, which are distinguished by their shape parameters. The type I distribution, also known as the Gumbel distribution, refers to the case where $\xi=0$; while the types II and III are known as the Fréchet distribution and the Weibull distribution corresponding to the cases where $\xi>0$ and $\xi<0$ respectively. These three parameters are invariable with time or other covariations, hence the name 'stationary'. The parameters of the stationary model (S) are estimated by using the Maximum Likelihood (ML) method (Myung, 2003) which is a common and robust choice.

In the stationary case, for any given year and a threshold $x_{0}$, the exceedance probability is $1-$ $F\left(x_{0}\right)$. The return period for $x_{0}$ (i.e., the number of years it takes for the exceedance event returns) can be calculated as $\tau=\frac{1}{1-F\left(x_{0}\right)}$. The link between $\tau$ and the number of expected exceedances $K$ over $T$ years, can then be explained by starting with a duration $d t$ with the corresponding number of exceedances $d K$ :

$$
d K=\frac{d t}{\tau}=\left[1-F\left(x_{0}\right)\right] d t
$$

and then integrating over $T$ years:

$$
K=\int_{0}^{T} d k=\int_{0}^{T}\left[1-F\left(x_{0}\right)\right] d t
$$

It should be noted that for the AMDR over $N$ years (113 years in the GB case and 129 years in the AU case), since $F\left(x_{0}\right)$ is time-independent, Eq. (4.8) leads to the normal finding: $K\left(x_{0}\right)=$ $\left[1-F\left(x_{0}\right)\right] N$, i.e., for $K$ exccendances over $N$ years, the return period is $N / K$. 


\subsubsection{Nonstationary generalized extreme value model (NS-GEV)}

Compared with the stationary model, the nonstationary model makes an important extension by assuming that the parameters change over time. In this study, the scale and location parameters are considered to vary with time and thus the cumulative probability is:

$$
F_{t}\left(x_{t} ; \sigma_{t}, \mu_{t}, \xi\right)=\exp \left[-\left(1+\xi\left(\frac{x_{t}-\mu_{t}}{\sigma_{t}}\right)\right)^{-1 / \xi}\right]
$$

Basically, the CDF $F_{t}$ of the NS-GEV follows the same form as the stationary one with an additional subscript $t$ added to the location and scale parameters which indicates that both parameters are time-dependent.

Table 4.1 Stationary and nonstationary GEV models and the estimation methods.

\begin{tabular}{|c|c|c|}
\hline Description & Parameters & Estimation Method(s) \\
\hline Stationary model: $F\left(x ; \sigma_{0}, \mu_{0}, \xi\right)$ & $\sigma_{0}, \mu_{0}, \xi$ are constant & ML* $^{*}$ \\
\hline Nonstationary model 1: & $\mu_{t}=\mu_{0}+\mu_{1} \times t$ & \multirow{2}{*}{ ML and B-MCMC* } \\
$F_{t}\left(x_{t} ; \sigma_{0}, \mu_{t}, \xi\right)$ & $\sigma_{0}, \xi$ are constant & \\
\hline Nonstationary model 2: & $\sigma_{t}=\sigma_{0}+\sigma_{1} \times t$ & \\
$F_{t}\left(x_{t} ; \sigma_{t}, \mu_{t}, \xi\right)$ & $\mu_{t}=\mu_{0}+\mu_{1} \times t$ & ML and B-MCMC \\
\hline Nonstationary model 3: & $\xi$ is constant & \\
$F_{t}\left(x_{t} ; \sigma_{t}, \mu_{t}, \xi\right)$ & $\sigma_{t}=\exp \left(\sigma_{0}+\sigma_{1} \times t\right)$ & \multirow{2}{*}{ ML and B-MCMC } \\
\hline
\end{tabular}

Noted that ML* is short for the "Maximum Likelihood" method and B-MCMC* is for the "Bayesian Markov-

Chain Monte-Carlo" method.

To create a stable quantile estimation consistent with the behaviour of rainfall extremes, four different GEV models are developed with different assumptions of parameters, as listed in Table 4.1. Both ML and the Bayesian Markov-Chain Monte-Carlo (B-MCMC) methods are employed to estimate the parameters of nonstationary models.

- The ML method

The ML method (Myung, 2003) is built upon the likelihood function of the occurrence of AMDR, which is the product of the probability density function of NS-GEV distribution: 


$$
L\left(x_{t} ; \boldsymbol{\theta}\right)=\prod_{t=t_{0}}^{t_{0+k}} f\left(x_{t} ; \boldsymbol{\theta}\right)=\left(\frac{1}{\sigma_{t}}\left(1+\xi\left(\frac{x_{t}-\mu_{t}}{\sigma_{t}}\right)\right)^{-\frac{1}{\xi}-1} \exp \left(-\left(1+\xi\left(\frac{x_{t}-\mu_{t}}{\sigma_{t}}\right)\right)^{-1 / \xi}\right)\right)^{k}
$$

The set of the parameters $\boldsymbol{\theta}$ can then be estimated by maximizing the likelihood function as $\frac{\partial L\left(x_{t} ; \boldsymbol{\theta}\right)}{\partial \boldsymbol{\theta}}=0$. It usually cannot be solved analytically, thus an iterative algorithm was employed to find the minimizer of $\frac{\partial L\left(x_{t} ; \boldsymbol{\theta}\right)}{\partial \boldsymbol{\theta}}$ starting with an initial guess which is based on the value of parameters estimated in stationary model S.

- The B-MCMC method

Not to get the parameters $\boldsymbol{\theta}$ of NS-GEV directly, the B-MCMC method makes use of Bayesian inference to estimate the posterior distribution of parameters $\boldsymbol{\theta}$ based on the informative prior knowledge. In this study, in order to ensure a better fit by taking full use of the knowledge, the estimated parameters of the stationary model were used to define the initial prior values of the NS-GEV model and the prior distribution of parameters is assumed to be a uniform distribution. Eq. (4.11) presents the transformation from the prior distribution to the posterior distribution by multiplying by its likelihood (Rasmussen and Ghahramani, 2003).

$$
p(\boldsymbol{\theta} \mid x, t) \propto p(x \mid \boldsymbol{\theta}, t) \times p(\boldsymbol{\theta} \mid t)=\prod_{t=\mathrm{t}_{0}}^{t_{0+k}} p\left(x_{t} \mid \boldsymbol{\theta}_{t}, t\right) \times p(\boldsymbol{\theta} \mid t)
$$

where $p(x \mid \boldsymbol{\theta}, t) \propto L(x ; \boldsymbol{\theta}, t)$ is the likelihood function and $p(\boldsymbol{\theta} \mid t)$ is the prior probability distribution of the parameters $\boldsymbol{\theta} ; t$ indicates the time from $t_{0}$ to $t_{0+k}$.

Numerical iterations for exploring the posterior distribution are carried out by using the MCMC simulation (Binder et al., 2012; Manly, 2018; Metropolis and Ulam, 1949), which is also aimed at analysing the uncertainty of the NS-GEV model. The final simulation results are compared with those estimated using the ML method.

The essence of the MCMC algorithm is to generate a trial moving from the current state of the Markov Chain with a prior probability of parameters $p(\boldsymbol{\theta} \mid t)$ to a next proposed state with a prior probability of the proposed parameters $p\left(\boldsymbol{\theta}^{\prime} \mid t\right)$. In this study, to make full use of the knowledge, the estimated parameters of the stationary model were used to define the initial prior values of the nonstationary parameters which are drawn from uniform distributions using 
Latin Hypercube Sampling (LHS). Numerical iterations for exploring the posterior distribution are carried out by using the MCMC simulation with Metropolis within Gibbs sampling. The Metropolis ratio is calculated to accept or reject proposal status and the convergence of simulation is monitored by Gelman-Rubin diagnostic (Gelman and Rubin, 1992).

This algorithm firstly starts as a random search over the entire prior distribution $(p(\boldsymbol{\theta} \mid t)$ of $D$ parameters using the LHS method then d samples are randomly assigned to $N$ Markov chains and the sample with the highest likelihood value will be selected as the starting point for each chain. To diversity the probability of the jumping direction, I broadly followed Sadegh et al. (2017) to use two approaches to update the chain: some chains (N1) follow the Adaptive Metropolis (AM) approach which is effective for searching direction at the early stage of MCMC and the rest (N-N1) follow the Differential evolution (DE) approach which has a stronger potential in converging to the target distribution. The details are shown below (Sadegh et al., 2017):

1) For each chain, randomly select $d$ samples from $D$ parameter spaces with Gibbs sampling (Gilks et al., 1995).

2) For N1 chains, propose a new state $S_{t+1}$ with a proposed set of parameters $\boldsymbol{\theta}^{\prime}$ by using AM approach, i.e., $S_{t+1}\left(\boldsymbol{\theta}^{\prime}\right)=S_{t}\left(\boldsymbol{\theta}^{\prime}\right)+(1-\beta) N\left(0_{\boldsymbol{\theta}^{\prime}}, \gamma_{1}^{2} \sum_{\boldsymbol{\theta}^{\prime}}\right)+\beta N\left(0_{\boldsymbol{\theta}^{\prime}}, \gamma_{2}^{2} I_{\boldsymbol{\theta}^{\prime}}\right)$;

For the rest N-N1 chains, the new state is $S_{t+1}\left(\boldsymbol{\theta}^{\prime}\right)=S_{t}\left(\boldsymbol{\theta}^{\prime}\right)+\gamma_{3}\left(S_{r_{2}}-S_{r_{1}}\right)+e$.

where $\sum_{\boldsymbol{\theta}^{\prime}}$ is the covariance matrix of $\boldsymbol{\theta}^{\prime}$ and $\beta$ is a random number in the range of $0 \sim 0.1 ; \gamma$ indicates the jump factors defined as $\gamma_{1}$ is a number randomly selected from $[1.2,2.2], \gamma_{2}=$ $2.38 / \sqrt{d}, \gamma_{3}=0.1 / \sqrt{d}$ (Roberts and Rosenthal, 2009) and $\gamma_{4}=2.38 / \sqrt{2 d}$ (Ter Braak, 2006); and $S_{r_{1}}$ and $S_{r_{2}}$ are two samplers drawn from parameter space D just for pre-defining the chain update direction.

3) Compute the Metropolis ratio $\frac{p\left(x \mid \boldsymbol{\theta}^{\prime}, t\right)}{p(x \mid \boldsymbol{\theta}, t)}$; if $\min \left(1, \frac{p\left(x \mid \boldsymbol{\theta}^{\prime}, t\right)}{p(x \mid \boldsymbol{\theta}, t)}\right) \geq p^{*}$, then accept $S_{t+1}$ and update the current chain where $p^{*}$ is the random number drawn from $N(0,1)$. If not, reject and go back to the previous step to re-propose the state. 
4) Check whether the iteration convergence or not by Gelman-Rubin convergence diagnostic.

To define the return period for the nonstationary case, the preceding procedure for the stationary case can be followed but have to be recognised as the time-varying nature of the nonstationary exceedance probabilities, i.e., for a given threshold $x_{0}$, the number of expected exceedances over $T$ years is:

$$
K_{t}\left(x_{0}\right)=\int_{0}^{T}\left[1-F_{t}\left(x_{0}\right)\right] d t
$$

For annual maxima, e.g., the AMDR series over $N$ years, this leads to

$$
K_{N}\left(x_{0}\right)=\sum_{i=1}^{N}\left[1-F_{i}\left(x_{0}\right)\right] \times 1
$$

where $F_{i}(\cdot)$ is the nonstationary CDF for the $i^{\text {th }}$ year. Correspondingly, the return period for the NS case is $\tau_{N}\left(x_{0}\right)=N / K_{N}\left(x_{0}\right)$. Note that a subscript $N$ is used here to indicate that fact that both the return level and the expected number of exceedances are dependent on the duration (the $N$ years).

\subsubsection{Goodness of fit and performance of the S-GEV and NS-GEV models}

The goodness of fit (GOF) of the fitted S-GEV model is further tested by two different methods: the Kolmogorov-Smirnov (KS) test (Kolmogorov, 1933; Smirnov, 1948) and AndersonDarling (AD) test (Anderson and Darling, 1952, 1954). The tests are carried out by comparing the empirical cumulative probability distribution of the datasets with the reference GEV cumulative probability distribution and the reference GEV distribution is selected by simulating 5,000 times by a bootstrap method (presented in Section 3.2.3 of Chapter 3).

For assessing the GOF of the nonstationary assumption, as well as comparing the performance between the S-GEV and NS-GEV models, firstly the difference measure (DIFF) is proposed and defined as the difference between the modelled AMDR $\left(y^{\prime}\right)$ either by the S-GEV or NSGEV model and the actual $\operatorname{AMDR}(y)$, as shown below:

$$
\mathrm{DIFF}=y^{\prime}-y
$$


The boxplot is generated based on the DIFF, which indicates the risk of underestimation $(\mathrm{DIFF}<0)$ or overestimation $(\mathrm{DIFF}>0)$ of extremes. Meanwhile, other three criteria are also applied to assess GOF of nonstationarity (Kim et al., 2017), i.e., the root mean squared error (RMSE), the Akaike Information Criterion (AIC; Akaike et al., 1973) and the Bayesian Information Criterion (BIC; Schwarz, 1978). Small values of these three criteria or small absolute values of DIFF are used to indicate better performance for model selection.

$$
\begin{gathered}
A I C=-\frac{2}{N} \times L L+2 \times \frac{k}{N} \\
B I C=-2 \times L L+\log (N) \times k
\end{gathered}
$$

where $N$ is the number of data (113 for GB case and 129 for AU case), $L L$ is the log-likelihood of the model on these data and $k$ is the number of parameters (e.g., 3 for stationary model S, 4 for nonstationary model NS1 and 5 for NS2 and NS3).

\subsection{Results}

\subsubsection{Results of BBS-MK test}

To evaluate whether non-stationarity exists and the AMDR is influenced by the impact of climate change during the period of over 100 years in GB and AU, the BBS-MK test was applied and the test results are illustrated in Figure 4.1 by the indicator Kendall's tau. 


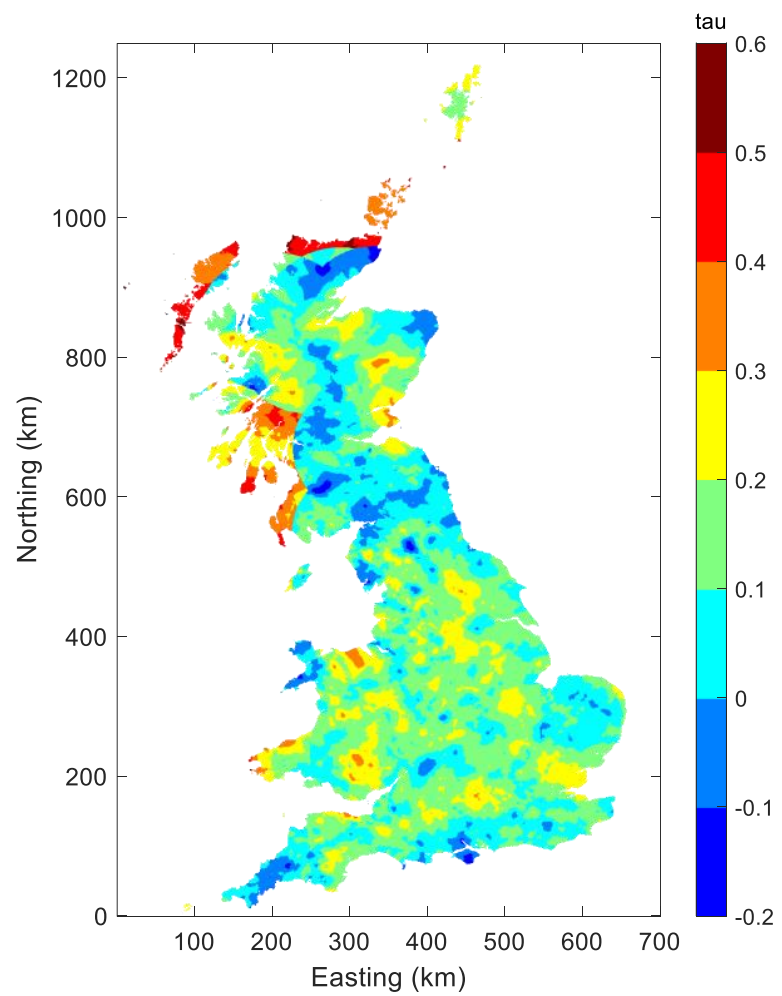

(a)

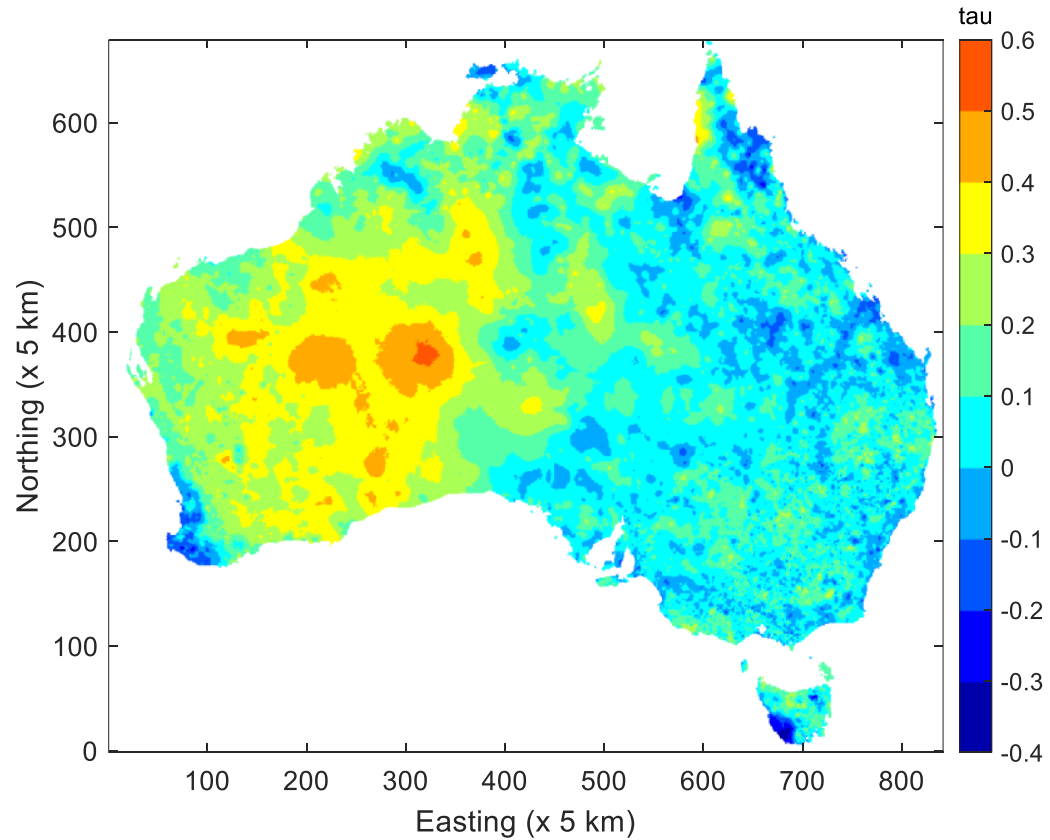

(b)

Figure 4.1 Trends of AMDR detected in GB (a) and AU (b), represented by Kendall's tau. 
The results present the nonstationary behaviour of both cases at the significant level of $5 \%$ where the reddish colour indicates the magnitude of increasing trend and bluish colour for decreasing trend, respectively. Most regions of middle England, Wales and western regions of Scotland are dominated by green, yellow, orange colours, indicating an increasing trend of AMDR. AMDR in north England and middle Scotland is shown an unchanged or decreasing trend. In AU, western regions are dominated by green and yellow colours while the middle and east regions are blue. It means that the magnitude of an increasing trend in the west is tested to be higher than east and AMDR in some coastal regions, e.g., east coasts, west-south coasts, is unchanged or even decreased. This demonstrates that the extreme daily rainfall is influenced by the impact of climate change in the study area and the presence of a statistically significant trend, therefore, violates the currently used stationary-based assumptions in the risk estimation in both two countries.

\subsubsection{Selection of stationary and nonstationary models and spatial nonstationary patterns}

The suitability of GEV is assessed using the bootstrapping KS and AD tests against the stationary GEV, and the results show that the GEV distribution fits well the AMDR series with a $100 \%$ pass of the KS test and more than $97 \%$ for the AD test presented in Figure 4.2. The best-fitted model of each ROI is selected by choosing the model with the smallest values of the criteria (RMSE, AIC and BIC). Results show that overall: 1) around 35\% of ROIs in GB prefer a stationary model while the rest $45 \%$ select NS1 (only $\mu$ is allowed to be time-varying) and $20 \%$ select NS2 and NS3 (NS2-3; both $\mu$ and $\sigma$ are time-varying); 2) AU has a relative lower ratio of ROIs favouring stationary model (around 20\%) while 50\% prefer NS1 and 30\% prefer NS2-3. As to the methods used to fit the preferred nonstationary models, the ML method performs better for $60 \%$ of the GB cases compared with $40 \%$ performed by the B-MCMC method. In the AU cases, the ML method is significantly more dominating, e.g., with a ratio of $90 \%$ vs $10 \%$. 

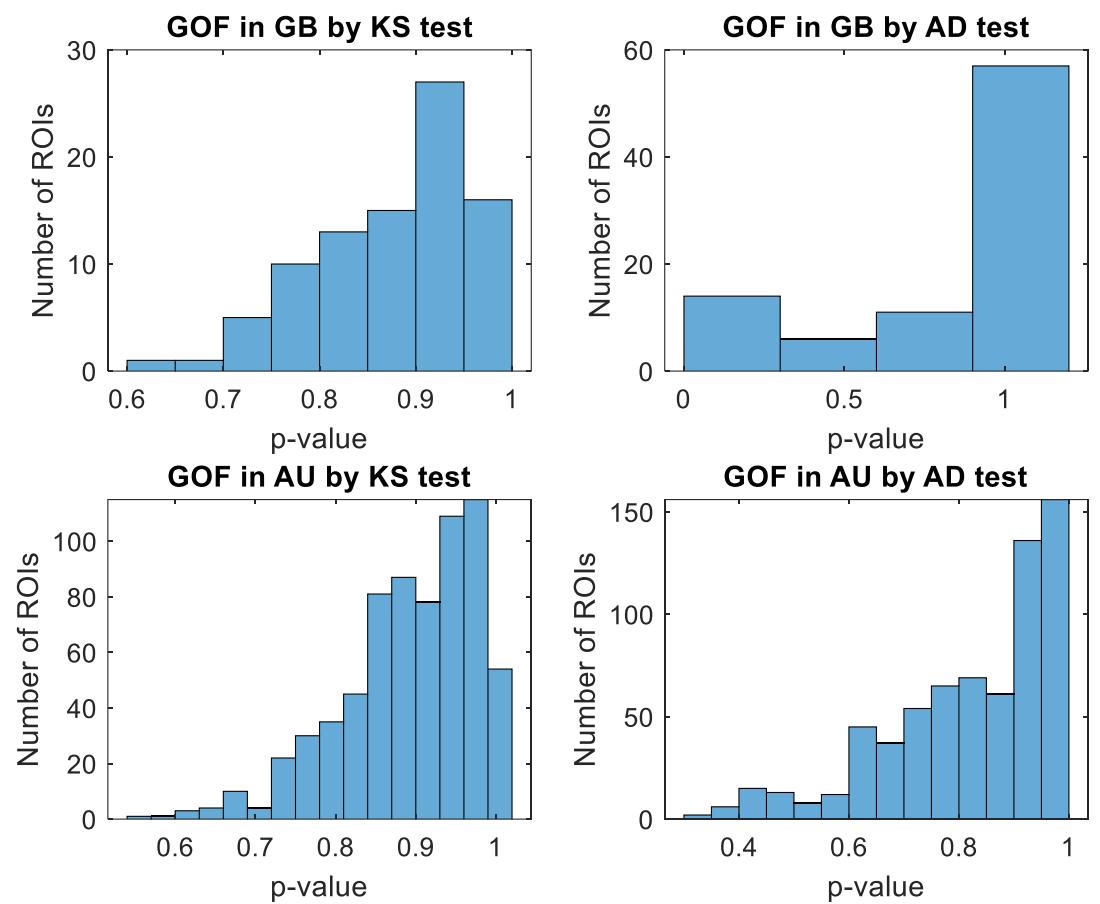

Figure 4.2 -values of all ROIs for temporary variation analysis in GB and AU.

The spatial distribution of model preference, i.e., stationary GEV versus nonstationary GEV is further demonstrated in subfigures a and e of Figure 4.3 where ROIs with the same size of 500 $\mathrm{km}^{2}$ and a relatively rounded shape is used. Geographically, those ROIs in GB that prefer nonstationary models are located along or near the coastal regions especially in eastern and northern GB and the Scotland Highland. In AU, nonstationary models dominate the inland area and the majority of south-western coastline while the north coastline of AU and the majority inland of Northern Territory favour stationary model.

As to the chosen types of GEV, subfigures $b$ and $f$ of Figure 4.3 present the spatial variation of the GEV types of the best-fitted models of these ROIs where the majority follows the Fréchet distribution. Out of all ROIs in GB, there are near $80 \%$ following the Fréchet distribution, mainly located inland; around 16\% following the Weibull distribution located on the western coast. In AU, around $90 \%$ of ROIs follow the Fréchet distribution and only a very small proportion (3\%) follows the Gumbel distribution. 


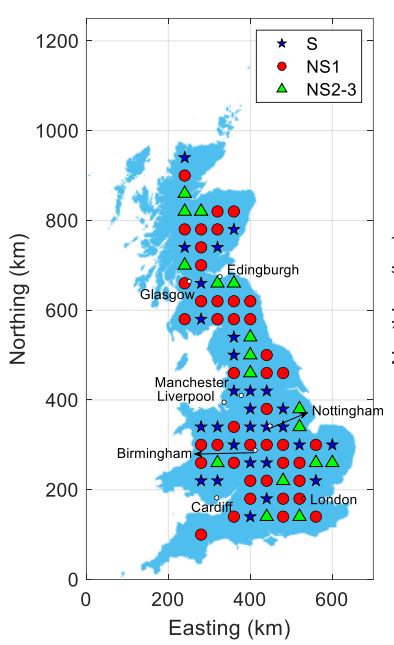

(a)

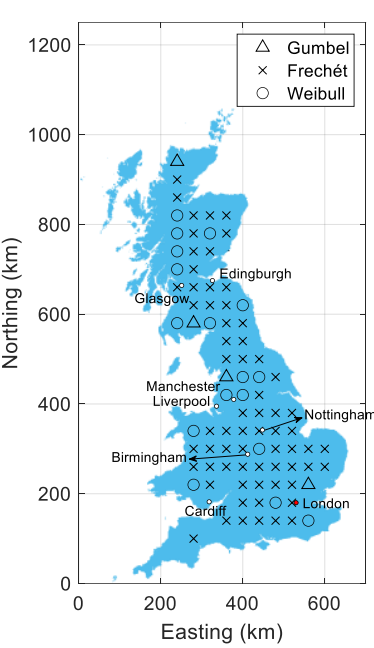

(b)

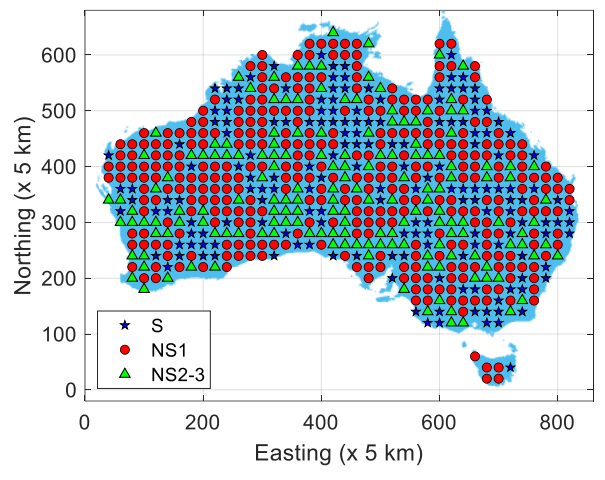

(e)

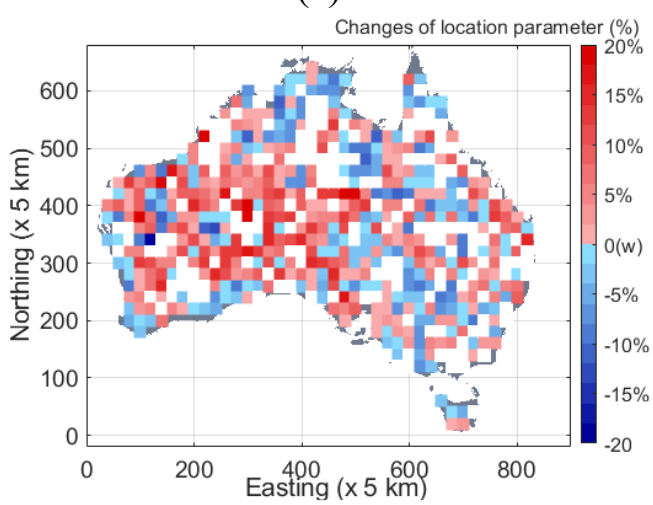

(g)

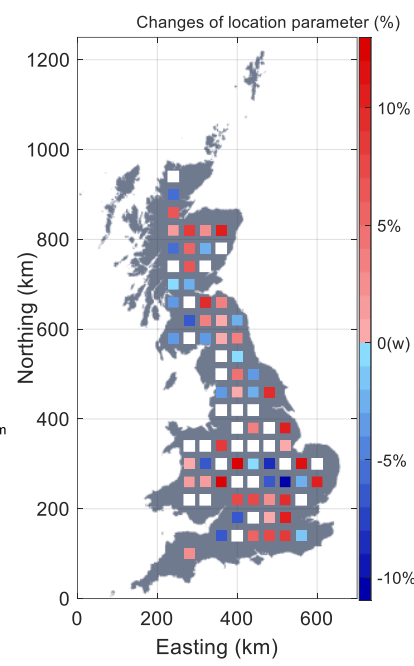

(c)

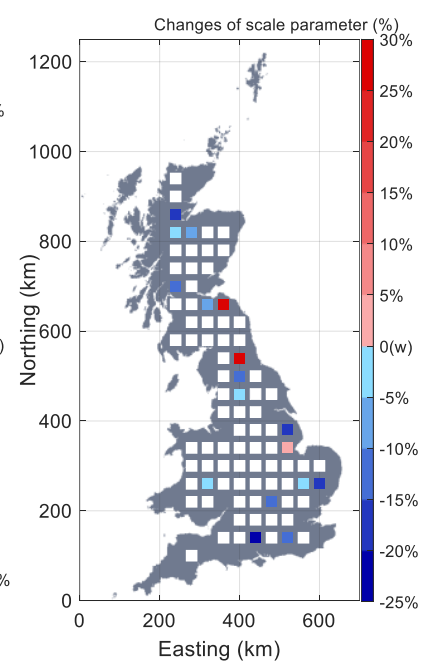

(d)

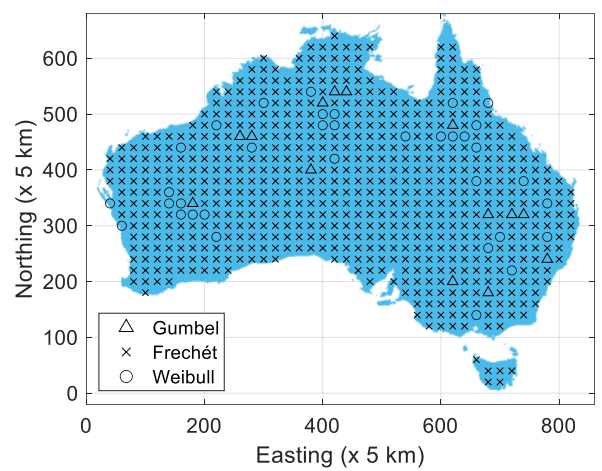

(f)

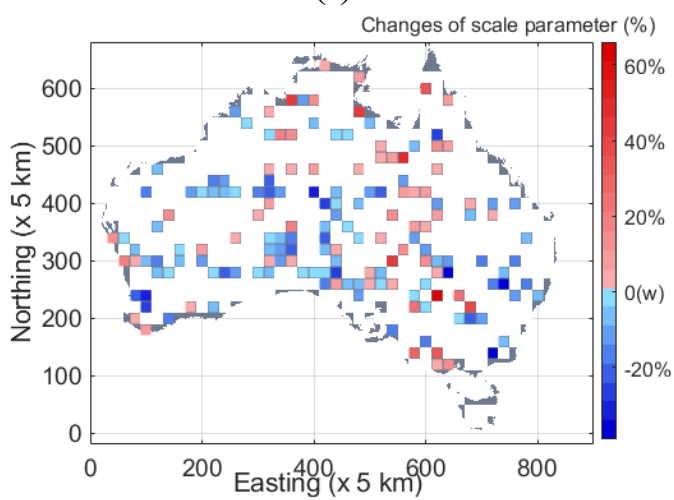

(h)

Figure 4.3 Spatial distribution of ROIs with the size of $500 \mathrm{~km}^{2}$ and relatively rounded shape in terms of (1)the best-selected model type in GB (a) and AU (e); (2) the best fitted GEV type in GB (b) and AU (f); (3) the changes of location ( $\mathrm{c}$ and $\mathrm{g}$ ) and scale parameters $(\mathrm{d}$ and $\mathrm{h}$ ) in percentage within the record periods (113 years 
for GB and 129 years for AU) and please noted that white colour ("w" shown in the colour bar) indicates the ROI with no change of the parameters.

To reveal the time-varying changes of $\mu$ and $\sigma$ of the best selected GEV model, the rate of the change at the end of the study period (i.e., 2010 for GB and 2018 for AU) is calculated with respect to the starting times (i.e., 1898 for GB and 1990 for $\mathrm{AU}$ ). In GB, the changes of $\mu$ are in the range of $\pm 10 \%$ and the ROIs with a decreasing $\mu$ are mainly located in southern Scotland and the regions between London and Birmingham while the majority areas show a non-decreasing $\mu$ which indicates that the level of most frequent AMDR is non-decreasing. However, in AU, the south-middle zone and the eastern coasts are dominated by an increasing $\mu$ up to the rate of $+20 \%$ while the north coast of Northern Territory and west-south coast of Western Australia are controlled by a decreasing $\mu$ with the rate of $-5 \%$. The majority regions of GB and AU are observed to have a constant $\sigma$ while the rest region shows a decreasing $\sigma$ scattering near the coasts of England, which somehow indicates a decreasing occurrence probability of extremes.

\subsubsection{Spatial variation of nonstationary patterns over ROI size}

In GB, the proportion of ROIs preferring stationary model is gradually increased with the growth of ROI size $\left(60 \%\right.$ for ROI size $<100 \mathrm{~km}^{2} ; 65 \%$ for ROI size in $100 \mathrm{~km}^{2} \sim 500 \mathrm{~km}^{2}$ and $67 \%$ for ROI size $>500 \mathrm{~km}^{2}$ ). However, such proportion in AU is relatively stable and keeps around $25 \%$ for stationary models and $75 \%$ for nonstationary models, regardless of the ROI size.

To help the discussion, the rate of the change of GEV parameters is introduced alongside the incremental change of ROI size (i.e., $+20 \%$ each), denoted as $\Delta . \Delta$ is the Sen'slop (Sen, 1968) of the BBS-MK test applied to detect the changes of parameters over region size at the significant level of 0.05 and white colour $(\Delta=0)$ in Figure 4.4 indicates the insignificant change. With the increase of ROI size, the reddish colour represents a positive $\Delta$ which means the parameter increases as well, while the bluish indicates the negative cases. Four parameters analysed are: 1) $\mu_{0}$ and $\sigma_{0}$ which indicate the baselines, i.e., the average estimation of the 
climate referring to the level of most frequent AMDR and the ocurrence probability of extremes; and 2) $\mu_{1}$ and $\sigma_{1}$ which are the time-varying changes from such baselines due to climate change while equal to zero if the best-selected model is stationary.

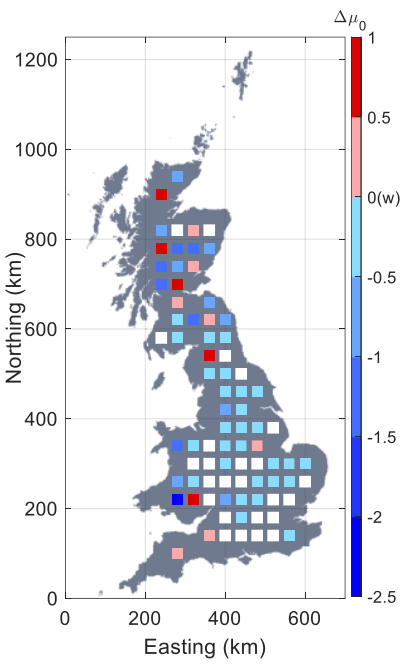

(a)

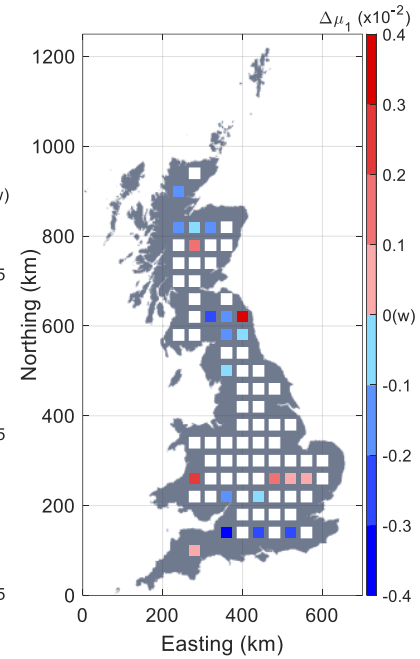

(b)

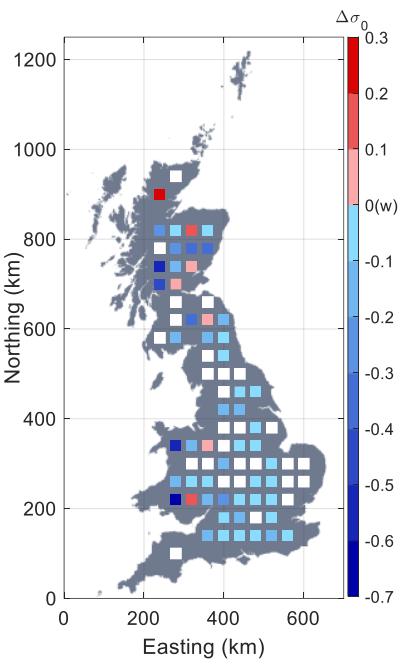

(c)

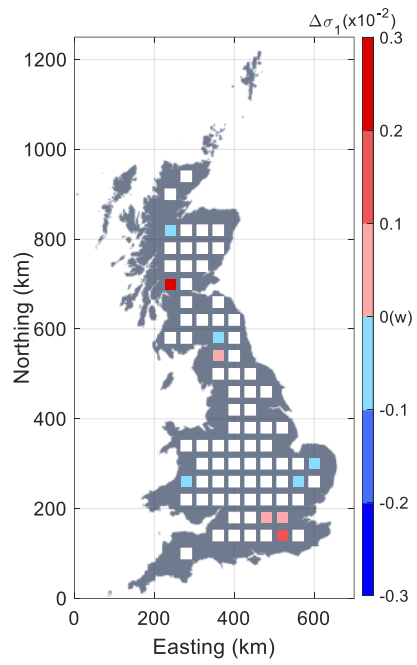

(d)

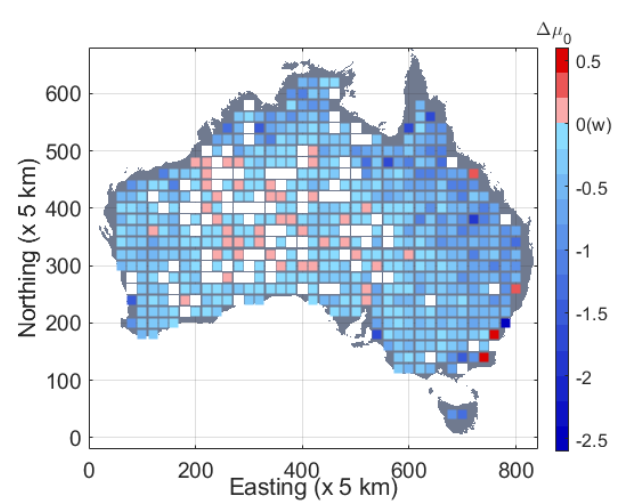

(e)

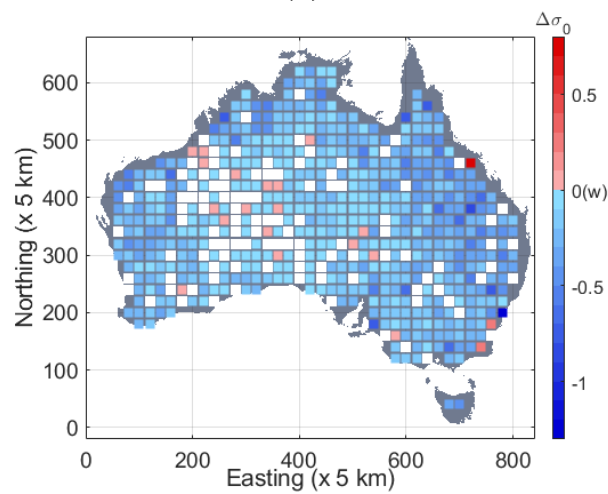

(g)

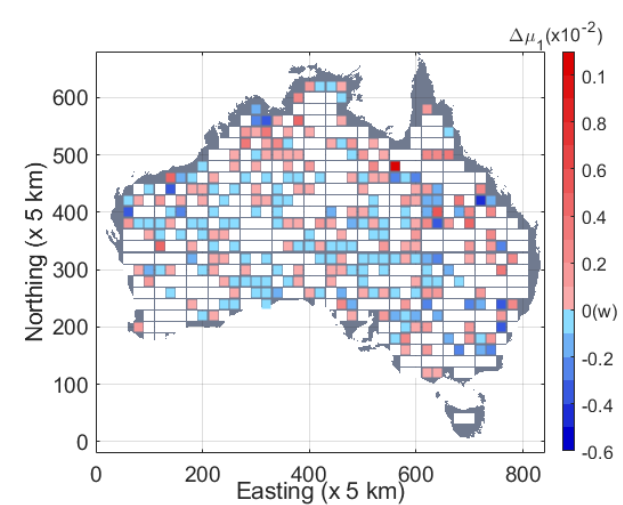

(f)

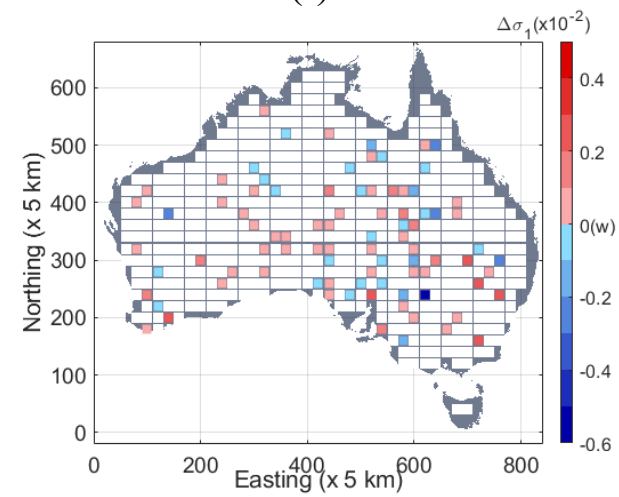

(h) 
Figure 4.4 Spatial distribution of ROI groups whose parameters $\left(\mu_{0}, \mu_{1}, \sigma_{0}, \sigma_{1}\right)$ change with the increase of ROI size in GB (abcd) and AU (efgh). Please note that the white colour indicates the ROI with the insignificant change of the parameters. Noted that "w" shown in the colour bar indicates the colour white which means the changes of the parameter is zero.

In both countries (Figure 4.4a\&c and Figure 4.4e\&g), most regions show decreasing baselines $\left(\mu_{0}, \sigma_{0}\right)$ as the ROI size increases, especially in AU. Such decrease can be attributed to the areal averaging when involving more grids in the sampled ROI. However, near the coastal regions of GB and boundary of the south-middle dry zone of AU, some ROIs do have increased baselines because the increasing size will involve more grids of higher rainfall which may overcompensate the reduction caused by the areal average.

In addition, the time-varying changes of parameters against size (i.e., $\mu_{1}$ and $\sigma_{1}$, see Figure $4.4 \mathrm{~b} \& \mathrm{~d})$ are insignificant in most parts of GB while very few with significant change locate near the coasts and have a decreasing $\mu_{1}$. In AU (Figure 4.4f), regions with a decreased $\mu_{1}$ are mainly located in the middle-south zone while the others with a increased $\mu_{1}$ are more closed to the coasts. However the spatial distribution of the changes of $\sigma_{1}$ is more random than $\mu_{1}$ and most regions present an insignificant trend of $\sigma_{1}$ in both countries.

The time-varying terms $\mu_{1}$ and $\sigma_{1}$ can reflect how the most frequent AMDR and the occurrence probability of extremes change over time affected by climate change in the last century. Interestingly, such impact is not always coincident with the decreased average climate estimation $\left(\mu_{0}\right.$ and $\left.\sigma_{0}\right)$ caused by a statistical average of larger ROI sizes, but influenced by the geographical locations, e.g., for the ROIs near the coasts in both countries, increasing their size can lead to an amplification of climate change impact on the most frequent AMDR and a higher probability for extremes to occur. However, in general, increasing region size will decrease both the average status of climate and climate change impact.

\subsubsection{Spatial variation of nonstationary patterns over ROI shape}

Figure 4.5 presents the changes of baselines $\left(\mu_{0}, \sigma_{0}\right)$ and time-varying terms $\left(\mu_{1}, \sigma_{1}\right)$ of the ROIs in GB and AU, parameterised by the ROI shape ( $s p)$. The shapes vary from an elongated 
west-east orientated $(s p=0.2,0.5)$, gradually to more rounded $(s p=0.8,1.0,1.25)$, then to an elongated but north-south orientated $(s p=5.0,2.0)$. A small difference is found between the baseline parameters of ROIs with reciprocal shape indexes especially in AU, e.g., two shapes with $s p$ of 0.5 and 2.0, which is regarded as a symmetric pattern around $s p=1.0$. However, such pattern is insignificant in the time-varying changes.

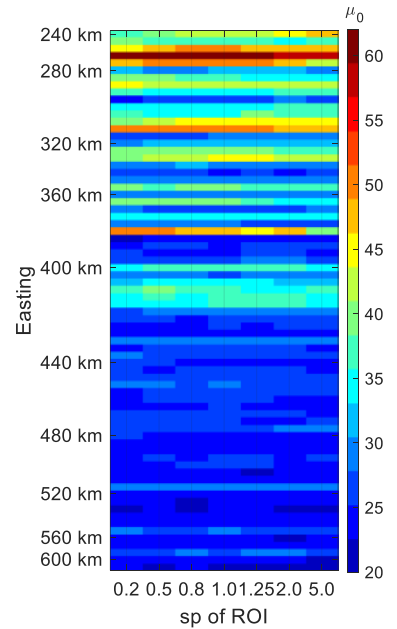

$\mathrm{sp}$ of ROI

(a)

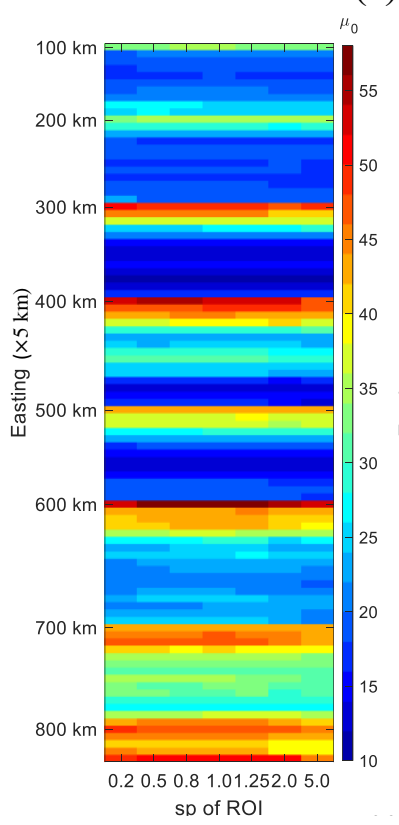

(c)

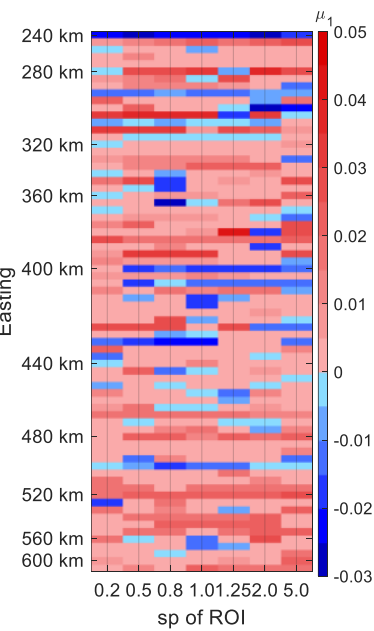

$\mathrm{sp}$ of ROI

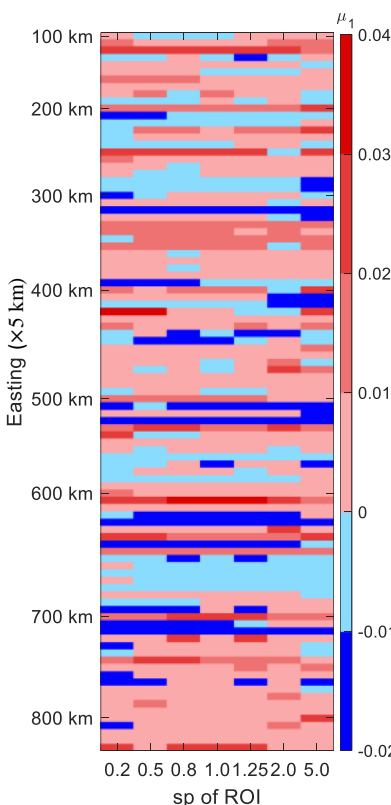

$\mathrm{sp}$ of $\mathrm{ROI}$

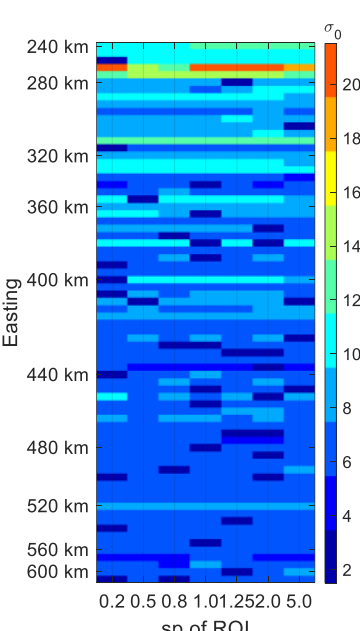

sp of ROI

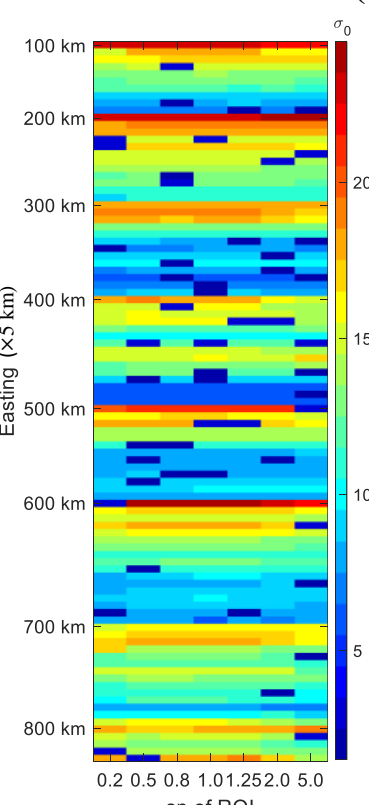

$\mathrm{sD}$ of ROI

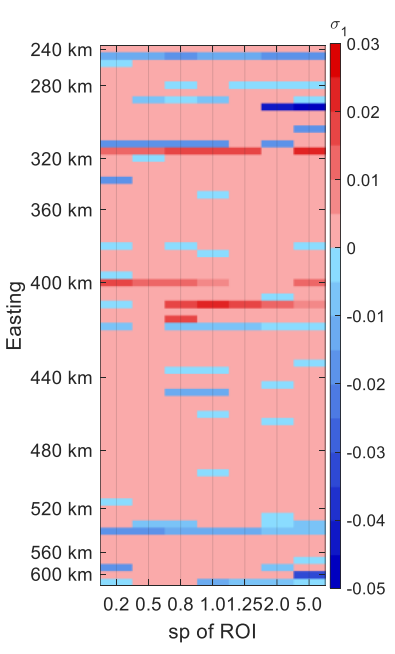

(b)

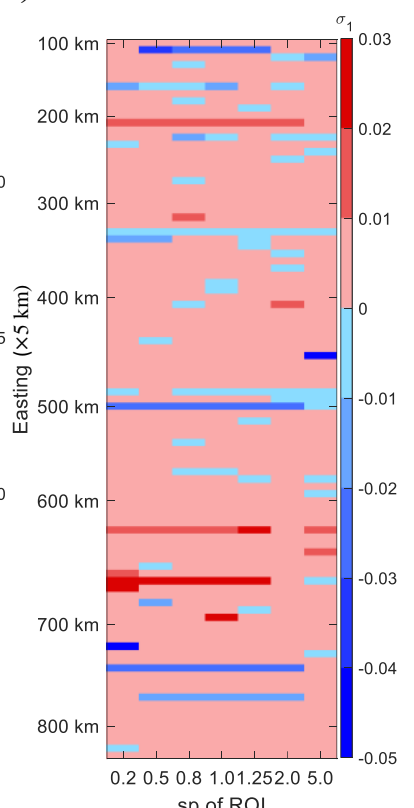

(d) 
Figure 4.5 Both baseline and time-varying parameters $\left(\mu_{0}, \mu_{1}, \sigma_{0}, \sigma_{1}\right)$ change over the ROI shape indicated by the index of $s p$ in GB (ab) and AU (cd). The horizontal axis indicates the location index of the ROIs and the colour bar shows the values of parameters.

\subsection{Implication on return period}

To demonstrate the difference between the nonstationary and stationary models, 6 reference return levels of $x_{0}$ are selected, i.e., $\mathrm{AMDR}_{\mathrm{ref} 2}, \mathrm{AMDR}_{\mathrm{ref5}}, \mathrm{AMDR}_{\mathrm{ref10}}$, $\mathrm{AMDR}_{\mathrm{ref25}}, \mathrm{AMDR}_{\mathrm{ref50}}$ and $\mathrm{AMDR}_{\mathrm{ref} 100}$ corresponding to the return periods of 2, 5, 10, 25, 50 and 100 years, and calculated from the stationary model. These stationary return levels are then applied to calculate their corresponding return periods under the nonstationary condition (see Eqs. (4.12) and (4.13)). The difference is then computed between the nonstationary return periods (NS-RP) and the referenced stationary return periods (S-RP) for the same given reference return levels.

Subfigures $\mathrm{a}$ and $\mathrm{b}$ of Figure 4.6 present the NS-RP estimated by their best selected nonstationary model for the three return levels $x_{0}$ from low to high (i.e., AMDR $\mathrm{A}_{\text {ref5 }}$, $\mathrm{AMDR}_{\mathrm{ref25}}$ and $\mathrm{AMDR}_{\mathrm{ref50}}$ ) in GB and AU. In both countries, the difference between NS-RP and S-RP can be ignored at the lower return level (e.g., $\mathrm{AMDR}_{\mathrm{ref5}}$ ). However, for a higher return level, the difference becomes significant. In GB, higher return levels (e.g., AMDR $_{\text {ref25 }}$ and $\mathrm{AMDR}_{\text {ref50 }}$ ) in North Wales, middle Scottish Highland and eastern and southern England (shown by blueish symbols) are underestimated by stationary model while the coastal regions of Scotland and Wales witness an overestimation. In AU, the middle region of New South Wales and the coastal region of North Territory are shown an underestimation on higher return levels by stationary model while the coastal area of Queensland and most regions of western Australia show an overestimation.

Generally, with an increase of the return periods, the difference between the return levels calculated by the nonstationary and stationary model grows larger (see boxplot in Figure 4.6c). In GB (the upper panel of Figure 4.6c), a left-skewed boxplot is observed at the higher return periods, which means that the return levels in most ROIs estimated by a stationary model are 
higher than the corresponding nonstationary model estimate; while in AU (the lower panel of Figure 4.6c), the difference is small and randomly distributed around zero and a half for overestimation and half for underestimation.

Combining the results with the best-selected models presented in Section 4.3.2, it can be seen that in GB, the AMDR in coastal regions, most part of Scotland and the east-south of England, the nonstationary condition is preferred and underestimated by stationary model, e.g., 1-in-50 year rainfall becomes 1 -in-30 year estimated by the best-fitted nonstationary model. In AU the same situation happens in the inland areas which are fitted better by the nonstationary model, such as the middle region of New South Walse and the boundary area with Queensland, north coastal regions of North Territory, but the underestimation is small, e.g., 1-in-50-years becomes 1-in-45-years.
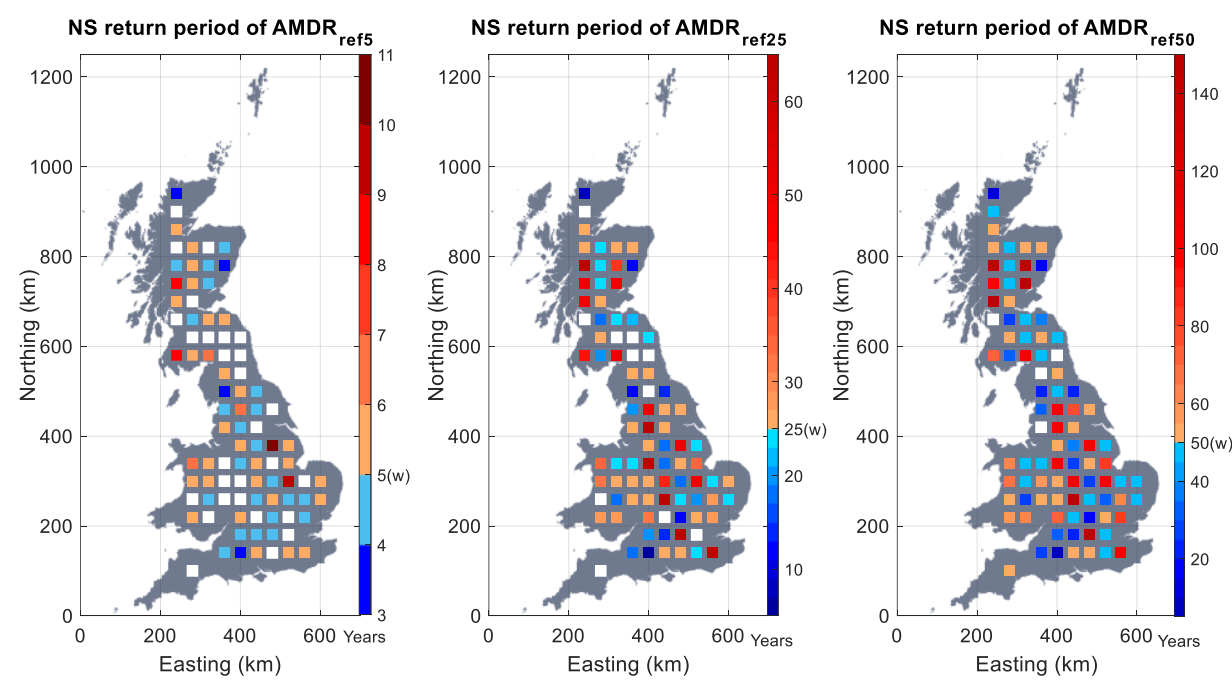

(a)
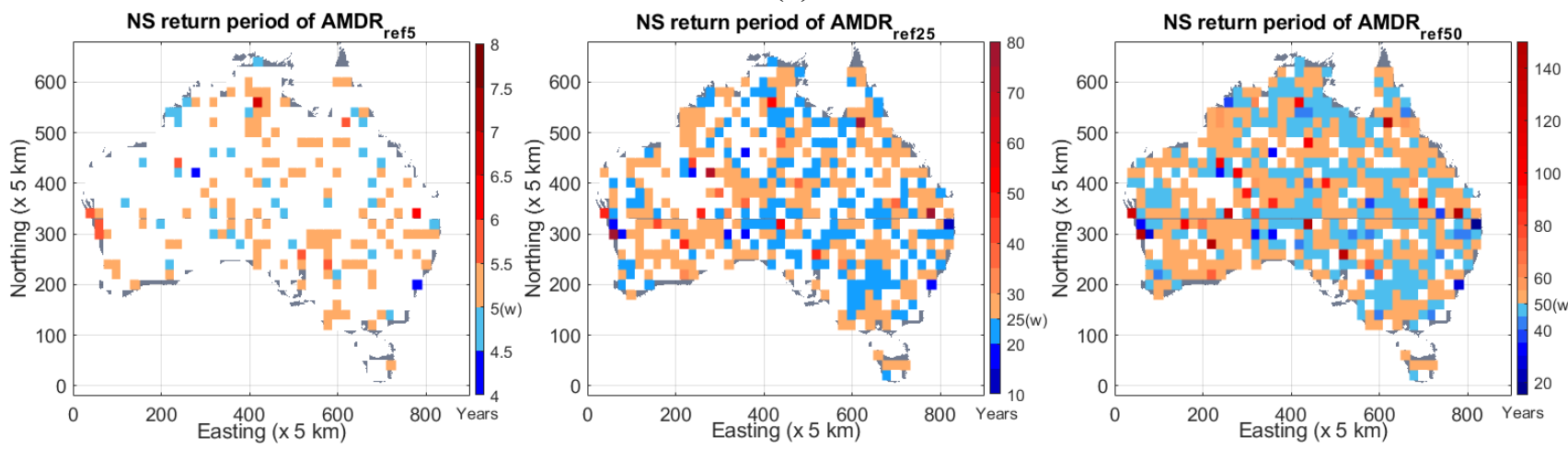

(b) 


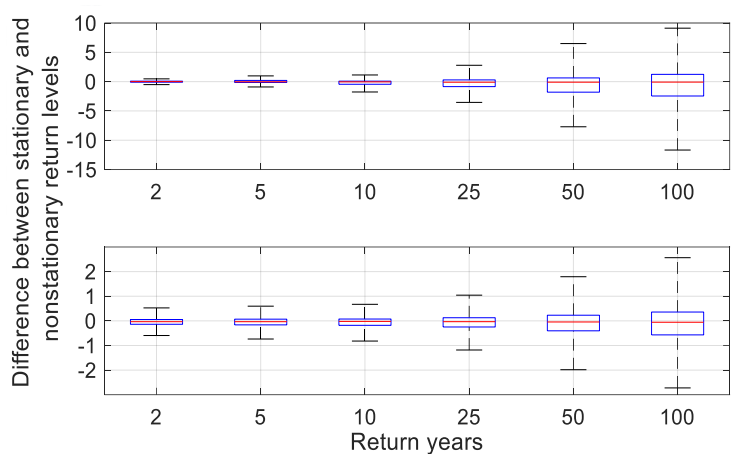

(c)

Figure 4.6 Nonstationary return periods corresponding to the return levels estimated by the stationary model and the spatial distribution referring to 1-in-5 years, 1-in-25 years and 1-in-50 years in GB (a) and AU (b). An overall comparison between the nonstationary and stationary return levels corresponding to the same return year is presented as a boxplot in c where the upper panel shows the GB case and the lower shows the AU case. Noted that "w" in the colour bar indicates the colour white which means there is no difference between stationary and nonstationary return periods.

\subsection{Summary and remarks}

This chapter moves forward from the results of Chapter 3 to present a study analysing the spatial variation of both the stationary and nonstationary GEV modelling of the annual maximum daily rainfall (AMDR) extracted from 11,011 regions of interest (ROIs, see Table 3.1 on Page 50 of Chapter 3) with different spatial properties (location, size and shape) in Great Britain (GB) and Australia (AU) using the same grid-based datasets. Three nonstationary models with different time-varying GEV parameters $(\mu$ and $\sigma$ ) schemes are proposed. They are fitted using both the Maximum Likelihood (ML) and the Bayesian Markov-Chain Monte-Carlo (B-MCMC) methods, before being compared with the stationary GEV models. Finally, the spatial patterns of the AMDR in both countries are analysed and quantified, with respect to the ROI's location, size and shape as well as the time-varying changes due to climate change. The following conclusions can be drawn:

1) In general, the majority of the ROIs in both countries (around 65\% in GB and $80 \%$ in AU) favour the nonstationary GEV (NS-GEV) model and most of them prefer the condition that only $\mu$ assumed to be linearly changing with time; most NS-GEV 
applications shows the ML method performs better than the B-MCMC method $(60 \%$ and $90 \%$ in GB and AU). AMDR of over $80 \%$ ROIs in both countries follows Fréchet distribution.

2) Geographic location is the most significant factor affecting not only the average status of climate (w.r.t. $\mu_{0}$ and $\sigma_{0}$ ) but also the time-varying changes due to climate change (w.r.t. $\mu_{1}$ and $\sigma_{1}$ ). During the last century in GB, the changes of the level of most frequent $\mathrm{AMDR}$ (w.r.t. $\mu$ ) are in the range of $\pm 10 \%$ and the majority areas show a nondecreasing trend. However, in AU, the south-middle zone and the eastern coasts are dominated by an increasing $\mu$ up to the rate of $+20 \%$ while the north coast of Northern Territory and west-south coast of Western Australia are controlled by a decreasing $\mu$ with the rate of $-5 \%$. The majority regions of GB and AU are observed a still $\sigma$ while some specific regions with a decreasing $\sigma$ scattering near the coasts of England indicates a decreasing occurrence probability of extremes.

3) Region size is the second factor and generally, the two countries show a decreased average status of climate with an increase of size because of statistical average. However, near the coastal regions of GB and the boundary of the south-middle dry zone of AU, some ROIs have an increasing status. Although the effect of region size on timevarying changes is insignificant, the climate change impact is not always decreased with the increase of region size, but is influenced by the geographical locations.

4) Region shape is not as significant affecting either the average climate status or timevarying changes; however, a symmetric pattern of average climate status is found for regions with reciprocal spatial indexes.

5) The stationary GEV models underestimate the risk in several specific regions such as the coastal regions in both countries where the nonstationary model is preferred. It may inspire a reconsideration of the current design storm determination procedure.

The findings from this chapter are valuable for the civil engineering community in a way that not only do they further corroborate other research findings on extreme rainfall, e.g. extremes are likely to become more frequent due to climate change impact, they also quantitatively 
address how such changes over not only the climate but also the geographical location, size and shape may affect the prevailing engineering design standard.

Further work is recommended to investigate closely the underlying datasets with respect to potential inconsistency in the resolution of the data observed near the West coast of Scotland and the AU coasts as well. In addition, a comparative study with long-term, single gauge observations, as well as catchment orientated sampling is likely to make conclusions more robust. 


\section{Chapter 5 Spatiotemporal Variation of Climate Projection Extremes Compared with Observations}

The studies discussed so far are all based on observation of hydroclimatic extremes; however, it is also important to explore the gap between climatic projection and observation, which is crucial for diagnosing or correcting the bias thereby improving the climate projection. To quantify the link between them in view of the nonstationary extreme nature and answer Q5, a methodological framework was proposed considering the distribution of three datasets of annual extreme daily precipitation over Great Britain: one is a dataset of observation and the other two are simulated using different climate models. In this chapter, the datasets are firstly converted to the same resolution and coverage, then the distributions of annual extremes extracted to both datasets are fitted by using generalised extreme value (GEV) distribution respectively. The Maximum likelihood and Bayesian Markov-Chain Monte-Carlo methods are introduced to estimate the parameters of the models under two scenarios in which 1) stationary distribution where all three parameters are constant and 2) nonstationary distributions assuming the location and scale parameters are changing with time. The goodness of fit and the convergence of fittings are tested and the correlation between those two pairs of parameters fitted from observations and simulations are analysed and quantified. 


\subsection{Introduction}

According to the latest climate reports released by the Met Office (Kendon et al, 2018), the rainfall of 2017 for the United Kingdom overall was $97 \%$ of the $1981-2010$ average and 102\% of the 1961-1990 average. The rainfall anomaly pattern showed considerable spatial variation with much of highland Scotland and lowland England drier than average. The wettest areas relative to average were in west Wales, north-west England and parts of south-west and northeast Scotland. Following this finding and considering the spatial resolution of the ERA20CM climate projection data (Table 1.1 on Page 20 of Chapter 1), three grids with the size of $0.4^{\circ} \times$ $3.15^{\circ}$ located in the midland of England, Scotland and Wales respectively are used to designate the study areas and to extract data. The locations of the three grids, i.e., study areas are shown in Figure 5.1.

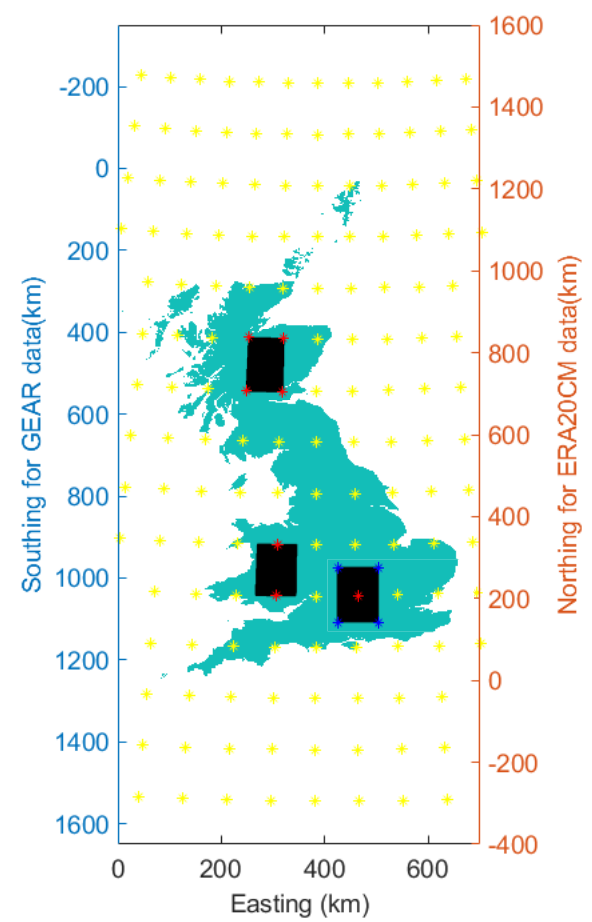

Figure 5.1 The location of three study areas (i.e., three grids of $0.4^{\circ} \times 3.15^{\circ}$ ). The green area corresponding to the left vertical axis shows the cover of GB and grids in the GEAR dataset while the yellow marks * corresponding to the right vertical axis indicate the edge of grids in the ERA20CM dataset. 


\subsection{Methodology}

The study is conducted by the following three steps which are also illustrated in Figure 5.2.

Step 1: process datasets to ensure consistency between the observed and the simulated rainfall data and select the length of years.

There are three datasets used, i.e., the GEAR dataset (observation) and the ERA20CM and UKCIP18 datasets (climate simulation). To match the spatial resolution, the National Grid Reference (NGR) used in the GEAR dataset is selected as the standard reference which adopts a transverse Mercator projection with an origin (the true origin) at $49^{\circ} \mathrm{N}, 2^{\circ} \mathrm{W}$ (an offshore point in the English Channel which lies between the island of Jersey and the French port of St. Malo) and the GEAR dataset generates a $700 \mathrm{~km}$ by $1300 \mathrm{~km}$ grid starting at the position of the false origin which is located $400 \mathrm{~km}$ west, $100 \mathrm{~km}$ north of true origin. For the ERA20CM dataset, I firstly project the longitude/latitude coordinate of the ERA20CM dataset to the National Grid Reference by the transverse Mercator projection method and then two datasets are merged into the same coordinates, then convert the 3-hour rainfall of the ERA20CM dataset to daily rainfall by aggregation. To keep the same spatial and temporal scales, the shorter length of the dataset is selected as the time period (i.e., the ERA20CM data of 111 years) and the areal annual maximum daily rainfall of two datasets from the year 1900 to 2010 is extracted from three selected study areas in England, Scotland and Wales for the study. However, for the UKCP18 dataset, as this dataset is recorded as latitude-longitude in rotated pole coordinates, I firstly rotate the coordinate back to be the same with the standard reference (i.e., same with the GEAR dataset) and do $1 \mathrm{~km}$ interpolation on the NGR. As the covered length of both datasets is 1981 to 2000,20 years are selected as the time period and the daily maxima of three selected areas are extracted from both datasets.

Step 2: simulate the areal annual maximum daily rainfall (AMDR) by both stationary and nonstationary GEV models and check the goodness of fits.

The same procedure for building stationary (S) and nonstationary (NS) GEV models is applied (see Sections 4.2.2 and 4.2.3 of Chapter 4). The stationary GEV model is estimated by the 
Maximum Likelihood (ML) method while the nonstationary GEV model is estimated using ML and Bayesian Markov-Chain Monte-Carlo (B-MCMC) methods respectively. The parameter assumption for the nonstationary GEV model is:

$$
\left\{\begin{array}{l}
\sigma_{t}=\sigma_{0}+\sigma_{1} \times t \\
\mu_{t}=\mu_{0}+\mu_{1} \times t
\end{array}\right.
$$

The goodness of fit of GEV assumption is tested by the KS and AD tests (see Sections 3.2.3 of Chapter 3) and RMSE is also involved to evaluate the simulation performance of two models, where its expression is given by:

$$
R M S E=\sqrt{\left(\sum_{i=1}^{n}\left(y_{i}^{\prime}-y_{i}\right)^{2}\right) / n}
$$

where $n$ is the total number of original extreme rainfall $y_{i}$ in the dataset and $y_{i}{ }^{\prime}$ is the corresponding simulation series by assumed GEV distribution. A sufficiently small amount of RMSE is also the criteria for convergence of MCMC simulation.

Step 3: analysing and quantifying the link between observed and climate projected extremes.

In this step, two GEV distributions are compared at different probability levels to find the links between in-situ observational extremes and climate projection extremes. 


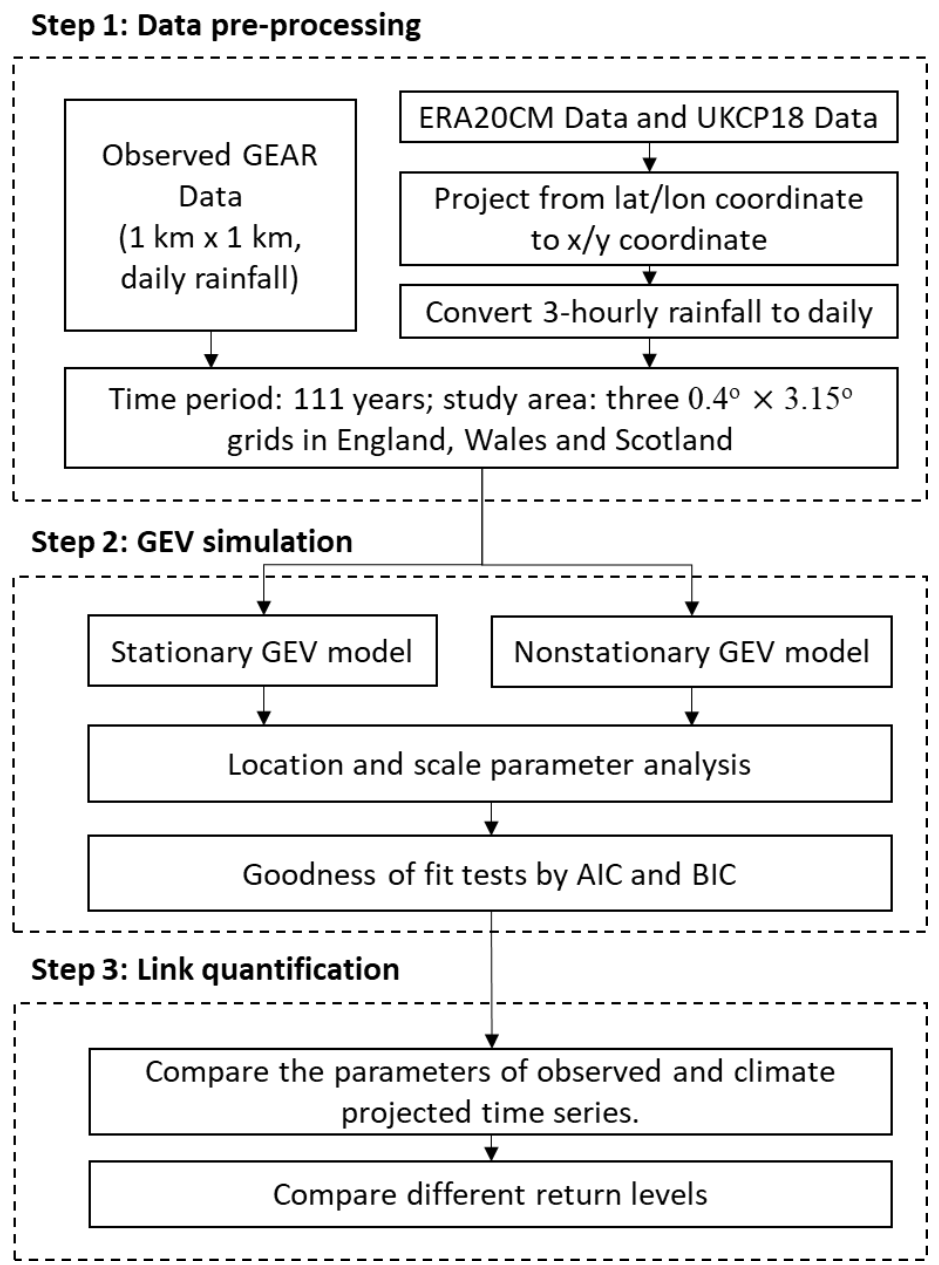

Figure 5.2 Methodology of the study.

\subsection{Results}

\subsubsection{Link to ERA20CM projected extreme rainfall in GB}

\section{- England}

Figure 5.3 present the comparison among stationary and nonstationary return levels for three time slices (i.e., the year 1910, 1960 and 2010) estimated by using different methods. Red circles indicate the observed AMDR from the GEAR dataset at its empirical return periods while grey circles are projected AMDR by 10 ensemble members of the ERA20CM dataset. It 
can be observed that more and more extreme events (e.g., high AMDR) are included as time moves on. For example, in the first 10 years (1900-1910), the number of events whose AMDR is higher than $35 \mathrm{~mm}$ is zero while such number increases to 1 until 1960 and has a dramatic increase in the following 50 years, i.e., 8 events until 2010. The stationary GEV cannot capture the extremes at the end of 2010 where around 5 red circles locate above the curve of stationary return level; however, both nonstationary models perform better than the stationary one on capturing the temporal change of AMDR and all red circles are below the return level curves by the end of 2010. Especially, the nonstationary model estimated by the ML method tends to overestimate the AMDR than the B-MCMC method.

S-GEAR/ERA20CM

(ML)
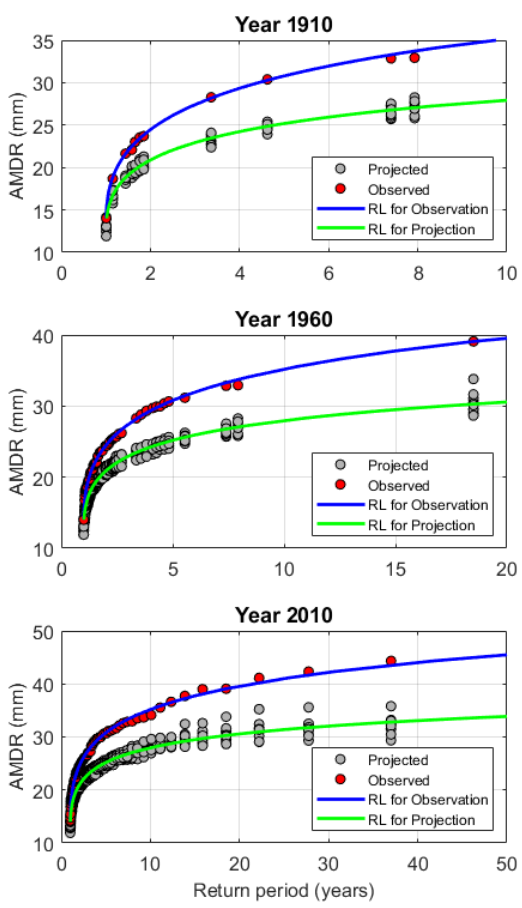

NS-GEAR/ERA20CM

(ML)
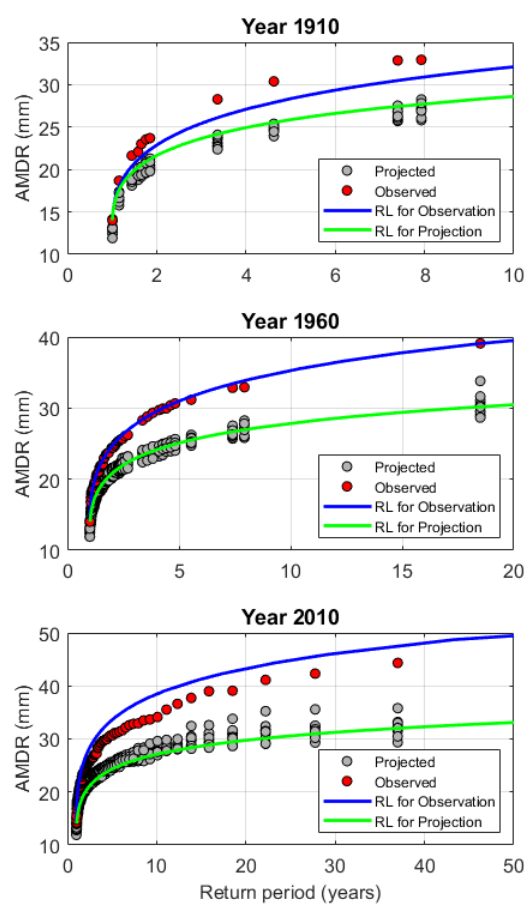

NS-GEAR/ERA20CM

(B-MCMC)
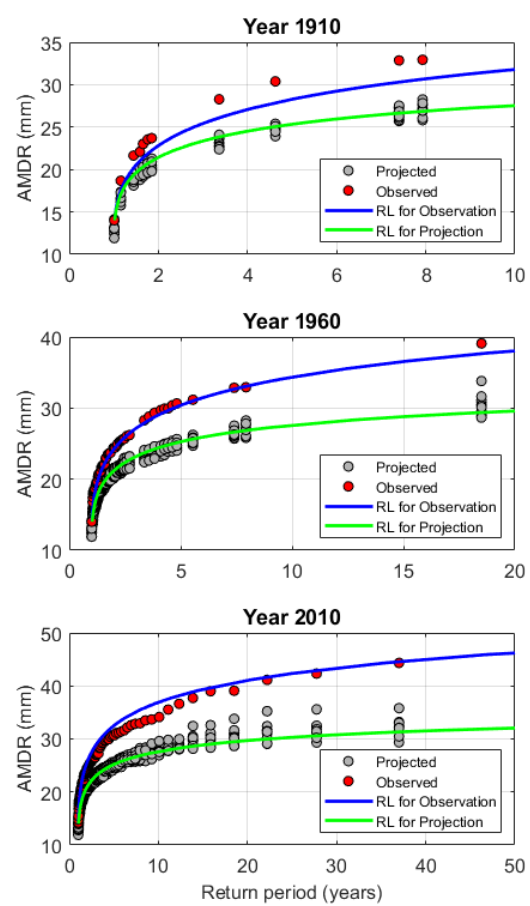

Figure 5.3 Stationary (S) vs. nonstationary (NS) return levels for three time slices (i.e., the year 1910, 1960 and 2010) estimated by different methods by using both the observed (GERA) and projected (ERA20CM) AMDR in the study area of England.

The green curves in Figure 5.3 are return levels simulated by averaging the 10 ensemble members of the climate projection model. Comparing these with the observed ones (e.g., those 
blue curves), it can be found that the climate projection model can only capture the AMDR at much lower levels, e.g., AMDR lower than 2-years return level, but significantly underestimate the AMDR at higher return levels regardless of both stationary and nonstationary assumptions.

To quantify the comparison results, the estimated parameters of both stationary and nonstationary models of both observed and projected AMDR in the study area of England are presented in Table 5.1. To simulate the observed AMDR time series, all three models perform well because of the small difference among RMSE values, however, the nonstationary model can capture the temporal changes of AMDR which are shown in Figure 5.3 where both $\mu$ and $\sigma$ increase over time, i.e., $\mu_{1}=0.023$ and $\sigma_{1}=0.013$. It means that the most frequent AMDR becomes higher and the occurrence probability of extreme events becomes larger. However, the projection data do not show such significant temporal changes of AMDR because the change of two parameters are closed to zero $\left(\mu_{1}=0.006\right.$ and $\left.\sigma_{1}=-0.003\right)$ according to the nonstationary simulation models. Comparing the values of parameters, i.e., $\mu_{0}(\mu)$ and $\sigma_{0}(\sigma)$, there is almost no difference between the $\mu$ 's from both the observed and projection datasets while $\sigma$ of the projected data is much smaller than that of the observed data. As the $\mu$ of GEV distribution indicates the value with the highest occurrence probability, i.e., the most frequent value while $\sigma$ somehow indicates a dispersion of values referring to $\mu$, it can be concluded that climate projection in England works better on simulating the most frequent annual maximum daily rainfall; however, it greatly underestimates the dispersion of extreme rainfall from the average, which underpins the fact that the climate projections need to be improved on simulating the differences between extreme and average.

Table 5.1 Estimated parameters of stationary (S) and nonstationary (NS) GEV models of both observed and projected AMDR in the study area of England.

\begin{tabular}{|c|c|c|c|c|c|c|c|c|c|}
\hline AMDR & $\begin{array}{c}\text { GEV } \\
\text { model }\end{array}$ & Estimator & $\sigma_{0}(\sigma)$ & $\sigma_{1}$ & $\mu_{0}(\mu)$ & $\mu_{1}$ & $\xi$ & $\begin{array}{c}\text { RMSE } \\
(\mathrm{mm})\end{array}$ & AICc \\
\hline \multirow{2}{*}{$\begin{array}{c}\text { Observed } \\
(\text { GEAR) }\end{array}$} & $\mathrm{S}$ & $\mathrm{ML}$ & $(5.32)$ & - & $(22.49)$ & - & 0.053 & 1.33 & 734.97 \\
\cline { 2 - 10 } & $\mathrm{NS}$ & $\mathrm{ML}$ & 4.51 & 0.014 & 20.52 & 0.031 & 0.040 & 1.81 & 738.35 \\
\cline { 2 - 10 } & $\mathrm{B}-\mathrm{MCMC}$ & 4.84 & 0.013 & 20.77 & 0.023 & -0.031 & 1.27 & 734.28 \\
\hline \multirow{2}{*}{$\begin{array}{c}\text { Projected } \\
\text { (ERA20CM) }\end{array}$} & $\mathrm{S}$ & $\mathrm{ML}$ & $(3.89)$ & - & $(19.46)$ & - & -0.027 & 0.71 & 655.32 \\
\cline { 2 - 9 } & $\mathrm{NS}$ & $\mathrm{ML}$ & 3.80 & 0.001 & 20.42 & -0.017 & -0.022 & 0.87 & 662.75 \\
\cline { 2 - 10 } & $\mathrm{B}-\mathrm{MCMC}$ & 3.94 & 0.003 & 20.07 & -0.006 & -0.154 & 0.61 & 657.70 \\
\hline
\end{tabular}




\section{- Scotland}

Similar to Figure 5.3, Figure 5.4 present the comparison among stationary and nonstationary return levels for the three time slices in Scotland. Unlike the England case, in the first 10 years (1900-1910), the number of events whose AMDR is higher than $50 \mathrm{~mm}$ is only 1 then the number has a dramatic increase to 8 during the period of 50 years (1910-1960) and 10 in the last 50 years (1960-2010). In other words, although the trend of the number of extreme events increases, the growth rate is almost stable, and the number is almost the same comparing the first and second 50 years. Thus, the stationary GEV model can simulate the unchanging or slightly changing AMDR time series very well although there is an underestimation that can be observed in 2010 where some red circles stay above the stationary return level curve. Similar to the England case, the climate projected AMDR of 10 ensemble members is all smaller than the observed AMDR.
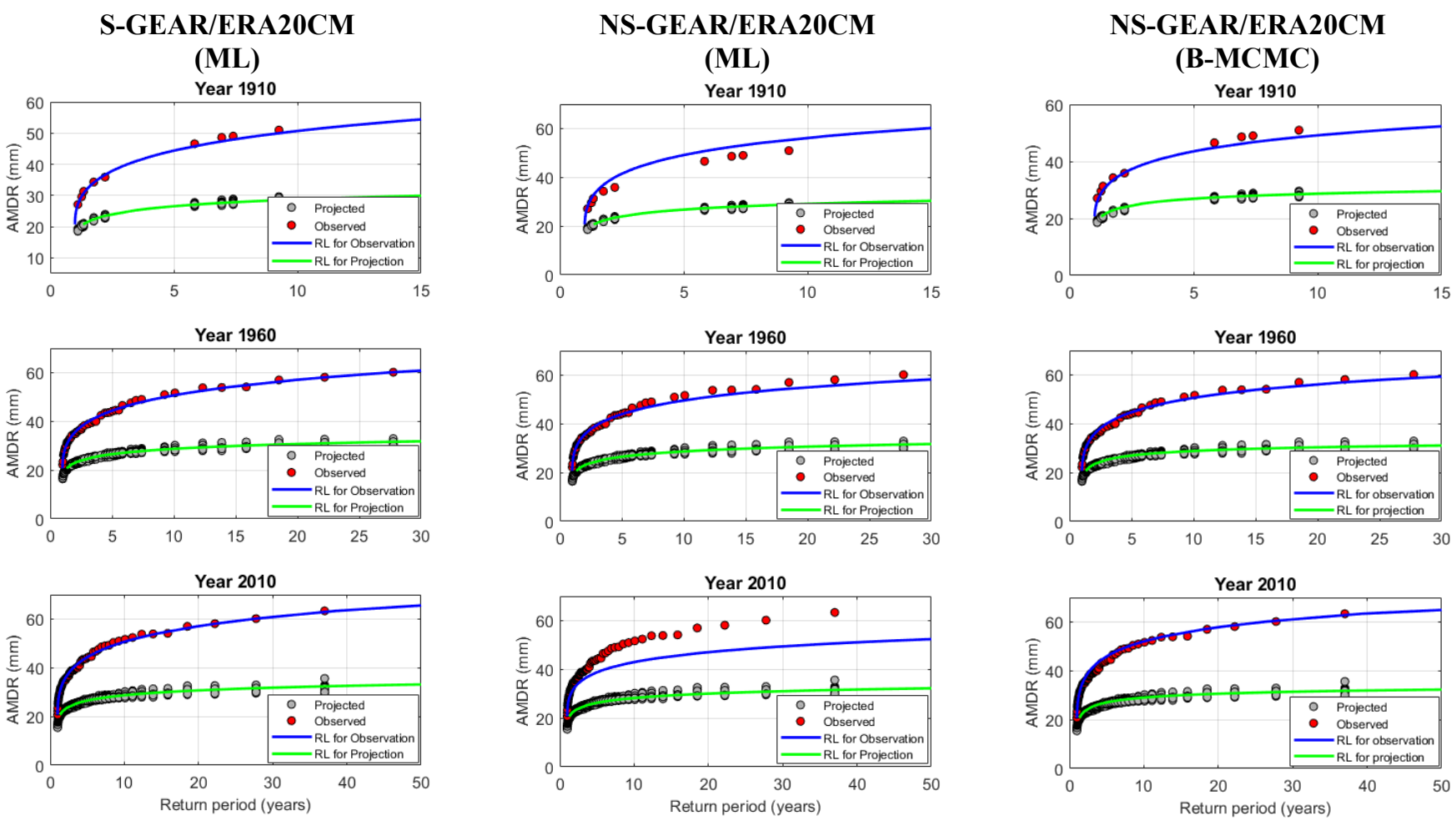
Figure 5.4 Stationary (S) vs. nonstationary (NS) return levels for three time slices (i.e., the year 1910, 1960 and 2010) estimated by different methods by using both the observed (GEAR) and projected (ERA20CM) AMDR in the study area of Scotland.

The parameters of both the stationary and nonstationary GEV models are shown in Table 5.2. For both cases, the B-MCMC method performs better in simulating the observed AMDR time series with small values of RMSE. Compared with the change of $\mu\left(\mu_{1}=0.014\right), \sigma$ is almost unchanged $\left(\sigma_{1}=0.007\right)$ which means the dispersion of the extreme rainfall is stable referring to an increasing most frequent AMDR during the past 111 years. However, the temporal change of parameters is very little indicated by the nonstationary model simulating climate projected $\operatorname{AMDR}\left(\mu_{1}=-0.004\right.$ and $\left.\sigma_{1}=0.004\right)$ and the stationary assumption can also fit very well.

Comparing the values of $\mu_{0}(\mu)$ and $\sigma_{0}(\sigma)$, climate projection data underestimate both two parameters, i.e., the scale parameter $\sigma$ is only half the value of $\sigma$ of the observed data while the location parameter $\mu$ is 10 times smaller than that of the model of the observed data. In other words, climate projection also greatly underestimates the most frequent extreme rainfall and cannot capture the difference between more extreme events and such most frequent levels in Scotland.

Table 5.2 Estimated parameters of stationary (S) and nonstationary (NS) GEV models of both observed and projected AMDR in the study area of Scotland.

\begin{tabular}{|c|c|c|c|c|c|c|c|c|c|}
\hline AMDR & $\begin{array}{c}\text { GEV } \\
\text { model }\end{array}$ & Estimator & $\sigma_{0}(\sigma)$ & $\sigma_{1}$ & $\mu_{0}(\mu)$ & $\mu_{1}$ & $\xi$ & $\begin{array}{c}\text { RMSE } \\
(\mathrm{mm})\end{array}$ & AICc \\
\hline \multirow{2}{*}{$\begin{array}{c}\text { Observed } \\
\text { (GERA) }\end{array}$} & $\mathrm{S}$ & $\mathrm{ML}$ & $(7.27)$ & - & $(32.81)$ & - & 0.077 & 2.30 & 806.96 \\
\cline { 2 - 10 } & $\mathrm{NS}$ & $\mathrm{ML}$ & 9.46 & -0.037 & 35.76 & -0.047 & 0.018 & 3.40 & 817.73 \\
\cline { 2 - 10 } & $\mathrm{B}-\mathrm{MCMC}$ & 7.60 & 0.007 & 31.98 & 0.014 & -0.011 & 1.51 & 804.88 \\
\hline \multirow{2}{*}{$\begin{array}{c}\text { Projected } \\
\text { (ERA20CM) }\end{array}$} & $\mathrm{S}$ & $\mathrm{ML}$ & $(3.32)$ & - & $(21.82)$ & - & -0.069 & 0.53 & 614.87 \\
\cline { 2 - 10 } & $\mathrm{NS}$ & $\mathrm{ML}$ & 3.62 & -0.005 & 21.66 & 0.003 & -0.072 & 0.65 & 628.46 \\
\cline { 2 - 10 } & $\mathrm{B}-\mathrm{MCMC}$ & 3.61 & 0.004 & 22.34 & -0.004 & -0.065 & 0.55 & 618.60 \\
\hline
\end{tabular}

- Wales

Similarities are also seen in the case of Wales. Figure 5.5 present the comparison among stationary and nonstationary return levels for the three time slices in the study area of Wales. In the first 10 years (1900-1910), the number of events whose AMDR is higher than $50 \mathrm{~mm}$ is 
only 1 then the number increase to 4 during the period of 50 years (1910-1960) and 10 in the last 50 years (1960-2010). It can be seen that an increasing trend of the extreme events, i.e., a net increase of 3 in the first 50 years and 6 in the next 50 years. Such increase is nonlinear which challenges the linear assumption of the nonstationary GEV models. As Figure 5.5 shown, both stationary model and nonstationary model estimated by using the B-MCMC method can perform well in the first 60 years but worse in the following 50 years where a lot of red circles locate beyond the blue curves. However, the nonstationary model estimated by using the ML method can capture the temporal change of extreme rainfall although there is a bit of overestimation in the year 2010. As to the climate projection dataset, all AMDR of 10 ensemble members is smaller than the observed ones.

S-GEAR/ERA20CM

(ML)
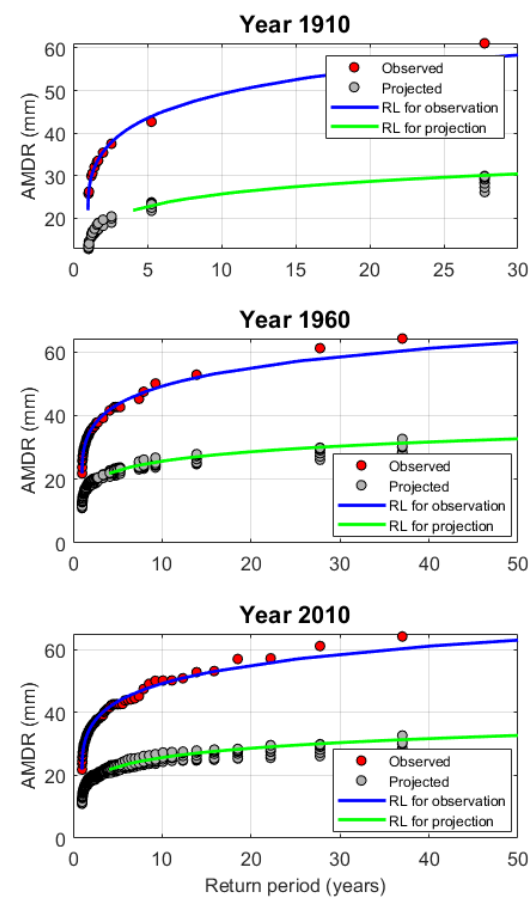

NS-GEAR/ERA20CM

(ML)
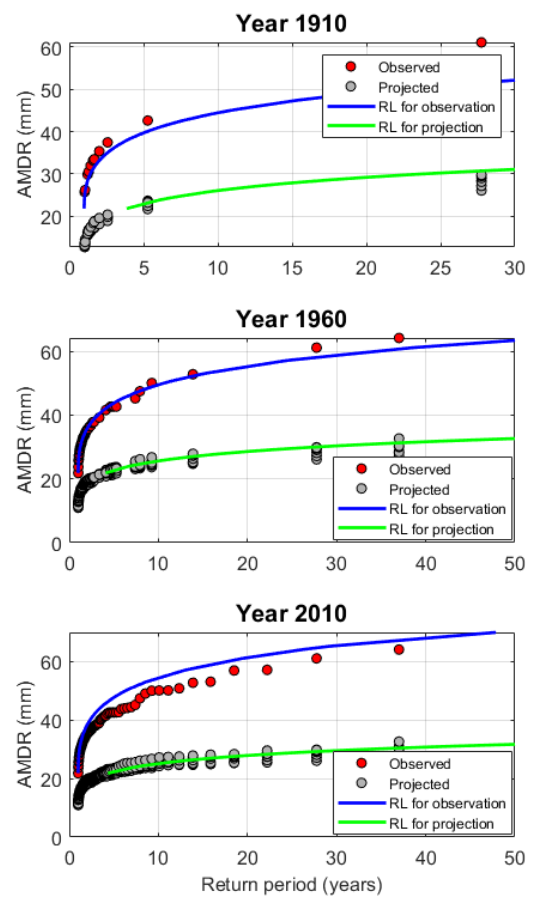

NS-GEAR/ERA20CM

(B-MCMC)
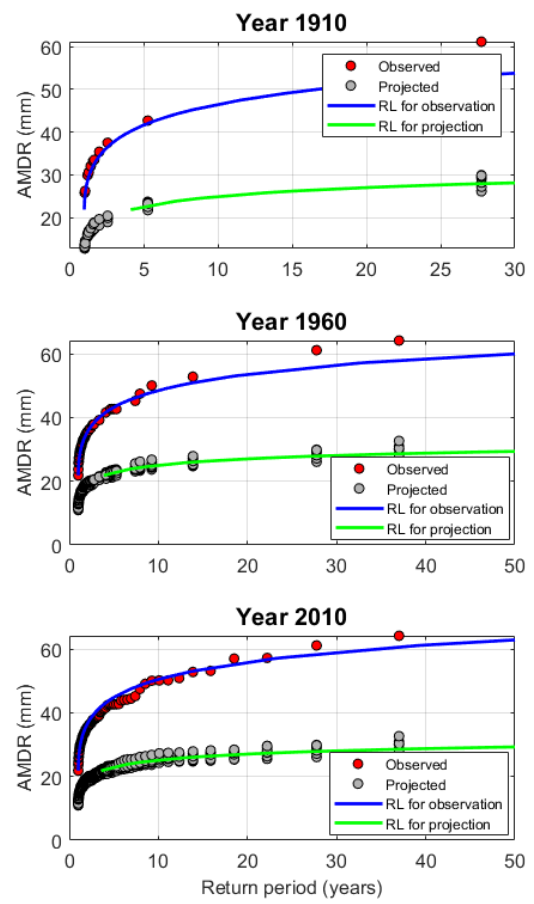

Figure 5.5 Stationary (S) vs. nonstationary (NS) return levels for three time slices (i.e., the year 1910, 1960 and 2010) estimated by different methods by using both the observed (GEAR) and projected (ERA20CM) AMDR in the study area of Wales. 
Table 5.3 illustrates the estimated values of parameters of both stationary and nonstationary GEV models for AMDR in the study area of Wales. Both models fitted using the B-MCMC method are shown to underestimate the observed AMDR time series. Although the RMSE is slightly higher, the nonstationary model estimated by the ML method can capture the changes of extreme rainfall at the end of 2010 with a little overestimation which will increase the cost but ensure the reliability of infrastructure. In other words, the stationary assumption may cause of failure of the defence structure as the increased number of extremes happened in Wales. Both parameters are estimated to increase over time, i.e., $\mu_{1}=0.044$ and $\sigma_{1}=0.023$ which are all higher than the study area of England and Scotland. It means the dispersion of the extreme rainfall is increasing referring to an increasing most frequent AMDR during the past 111 years in Wales. However, the temporal change of parameters of climate projected AMDR is insignificant indicating by nonstationary model simulation $\left(\mu_{1}=0.009\right.$ and $\left.\sigma_{1}=-0.004\right)$ and even an inverse change of scale parameter is observed. Comparing the values of $\mu_{0}(\mu)$ and $\sigma_{0}(\sigma)$, climate projection model underestimate both two parameters, i.e., the scale parameter $\sigma$ is only around half value of $\sigma$ of observed model while the location parameter $\mu$ is around 15 smaller than observed model. In other words, climate projection over-underestimates the most frequent extreme rainfall and cannot capture the difference between more extreme events and such most frequent levels in Wales.

Table 5.3 Estimated parameters of stationary (S) and nonstationary (NS) GEV models of both observed and projected AMDR in the study area of Wales.

\begin{tabular}{|c|c|c|c|c|c|c|c|c|c|}
\hline AMDR & $\begin{array}{c}\text { GEV } \\
\text { model }\end{array}$ & Estimator & $\sigma_{0}(\sigma)$ & $\sigma_{1}$ & $\mu_{0}(\mu)$ & $\mu_{1}$ & $\xi$ & $\begin{array}{c}\text { RMSE } \\
(\mathrm{mm})\end{array}$ & AICc \\
\hline \multirow{2}{*}{$\begin{array}{c}\text { Observed } \\
(\text { GEAR) }\end{array}$} & $\mathrm{S}$ & $\mathrm{ML}$ & $(6.55)$ & - & $(33.01)$ & - & 0.082 & 2.22 & 784.23 \\
\cline { 2 - 10 } & $\mathrm{NS}$ & $\mathrm{ML}$ & 5.18 & 0.023 & 30.65 & 0.044 & 0.083 & 2.73 & 788.29 \\
\cline { 2 - 10 } & $\mathrm{B}-\mathrm{MCMC}$ & 6.34 & 0.010 & 31.70 & 0.017 & 0.007 & 1.62 & 783.86 \\
\hline \multirow{2}{*}{$\begin{array}{c}\text { Projected } \\
\text { (ERA20CM) }\end{array}$} & $\mathrm{S}$ & $\mathrm{ML}$ & $(3.39)$ & - & $(17.34)$ & - & 0.073 & 1.11 & 638.01 \\
\cline { 2 - 9 } & $\mathrm{NS}$ & $\mathrm{ML}$ & 3.62 & -0.004 & 17.30 & 0.001 & 0.075 & 1.22 & 649.14 \\
\cline { 2 - 10 } & $\mathrm{B}-\mathrm{MCMC}$ & 3.91 & -0.004 & 17.09 & 0.009 & -0.111 & 0.65 & 641.98 \\
\hline
\end{tabular}

\subsubsection{Link to UKCP18 projected extreme rainfall in GB}

\section{- England}


Figure 5.6a presents the comparison between the observed and climate projected AMDR fitted by stationary GEV model and their PDF and CDF curves. It shows that the AMDR values from all ensemble members of the UKCP18 are greater than those from the observation at the same years. However, the deviation from their mean values keeps almost unchanged (seen as the parallel lines of the GEV modelled AMDR curves). In other words, UKCP18 estimates much better on the change of yearly AMDR although there is an overall overestimation on the mean AMDR. Therefore, the M4 is selected to represent the climate projection for comparison with the observations.

Figure 5.6b depicts the return levels calculated under both stationary and nonstationary assumptions whose corresponding models are estimated by ML and B-MCMC methods, shown for two specific years 1990 and 2000. In general, the temporal change of observed AMDR is not very significant during the 20 years because in the first 10 years (1981-1990), all events are lower than $35 \mathrm{~mm}$ while the last 10 years (1991-2000) only witnesses one event whose AMDR is higher than $35 \mathrm{~mm}$. By comparing both RMSE and AIC of the proposed GEV models, the best-selected model for modelling the AMDR from observation is nonstationary GEV estimated by B-MCMC method; for modelling the AMDR from climate projection is stationary GEV estimated by ML method, presented in Table 5.4. For the observed time series of AMDR in England, the deviation from the mean value is estimated to be increasing with time while the mean value (i.e., the most frequent AMDR) has a slight decrease for the 20 years. However, for the projected AMDR, the change of both parameters is insignificant for the 20 years. In conclusion, the UKCP18 projection outperforms the ERA20CM on simulating the observed AMDR in the same study area of England although the UKCP18 projection model overestimates the most frequent extreme rainfall and the difference between more extreme events and such most frequent levels in England. 

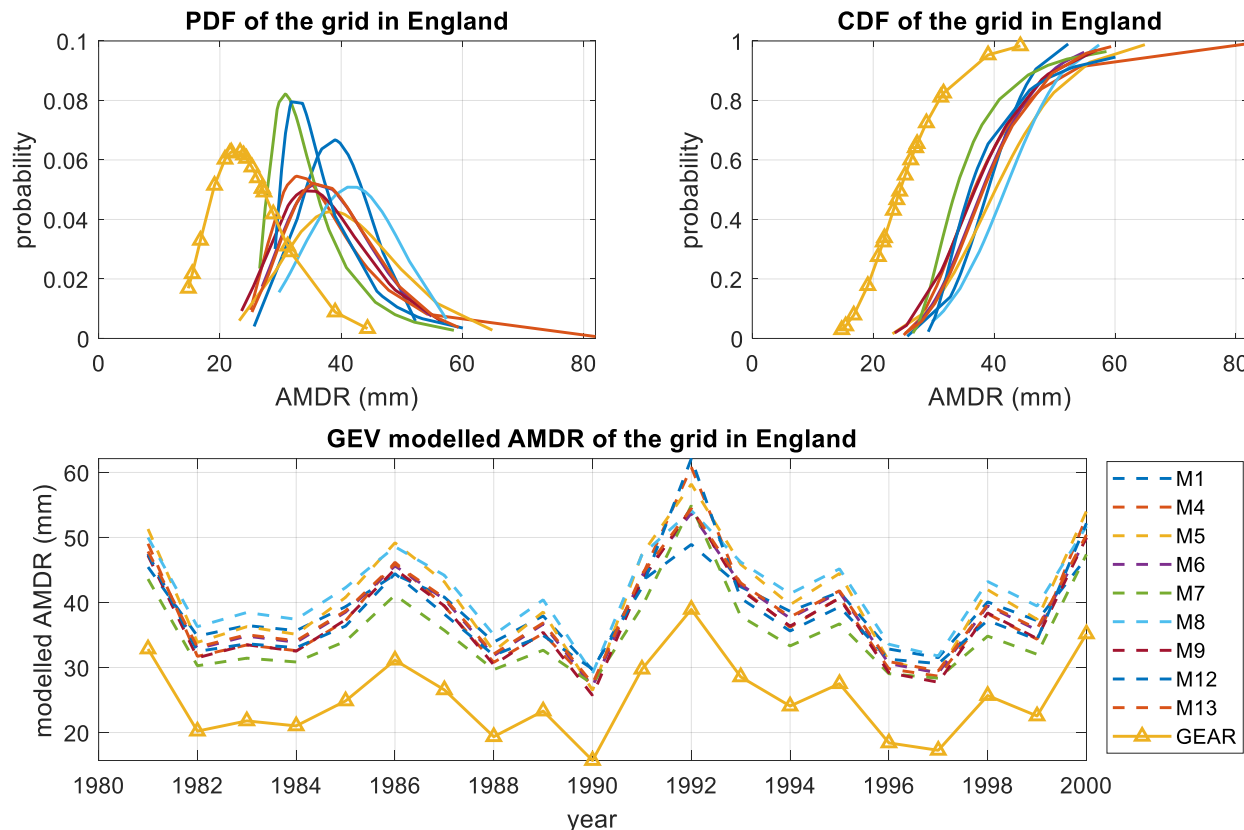

(a)

S-GEAR/UKCP18

(ML)
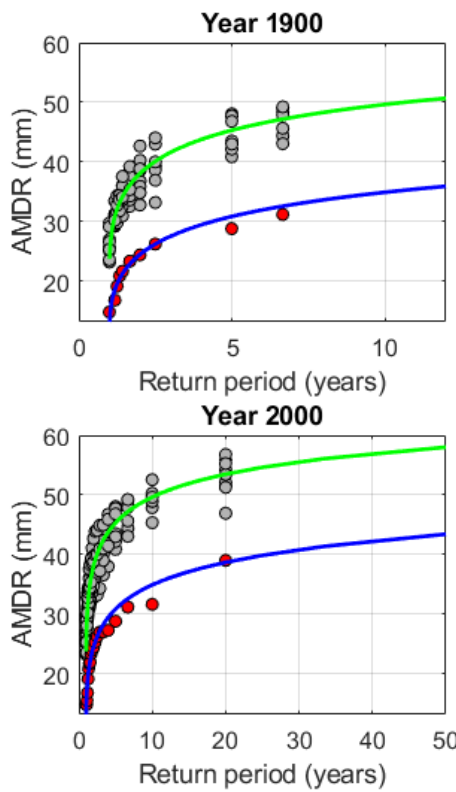

NS-GEAR/UKCP18

(ML)

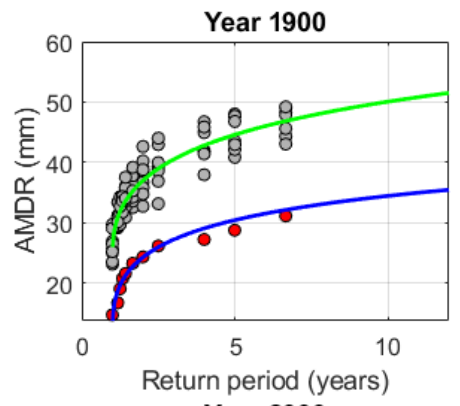

Year $\mathbf{2 0 0 0}$

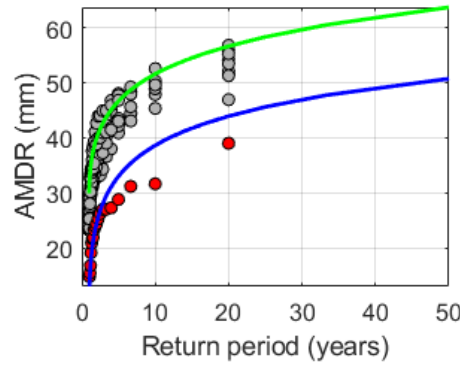

(b)
NS-GEAR/UKCP18

(B-MCMC)
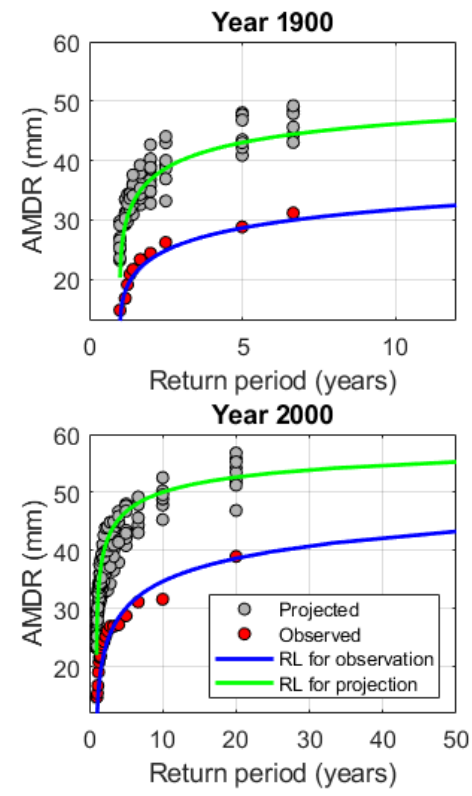

Figure 5.6 Comparison between stationary GEV of observed and climate projected AMDR in the study area of

England (a); and comparison of stationary (S) and nonstationary (NS) return levels of observed (GEAR) and climate projected (UKCP18) AMDR at the specific years 1990 and 2000 (b). 
Table 5.4 Estimated parameters of stationary (S) and nonstationary (NS) GEV models of both observed and projected AMDR in the study area of England.

\begin{tabular}{|c|c|c|c|c|c|c|c|c|c|}
\hline AMDR & $\begin{array}{c}\text { GEV } \\
\text { model }\end{array}$ & Estimator & $\sigma_{0}(\sigma)$ & $\sigma_{1}$ & $\mu_{0}(\mu)$ & $\mu_{1}$ & $\xi$ & $\begin{array}{c}\text { RMSE } \\
(\mathrm{mm})\end{array}$ & AIC \\
\hline \multirow{2}{*}{$\begin{array}{c}\text { Observed } \\
\text { (GEAR) }\end{array}$} & $\mathrm{S}$ & $\mathrm{ML}$ & $(5.83)$ & - & $(22.34)$ & - & -0.040 & 3.21 & 140.25 \\
\cline { 2 - 10 } & $\mathrm{NS}$ & $\mathrm{ML}$ & 3.05 & 0.245 & 23.15 & -0.083 & -0.016 & 3.79 & 146.87 \\
\cline { 2 - 10 } & $\mathrm{B}-\mathrm{MCMC}$ & 2.99 & 0.251 & 23.21 & -0.192 & -0.130 & 1.46 & 145.06 \\
\hline \multirow{2}{*}{$\begin{array}{c}\text { Projected } \\
\text { (UKCP18) }\end{array}$} & $\mathrm{S}$ & $\mathrm{ML}$ & $(7.08)$ & - & $(35.50)$ & - & -0.109 & 3.57 & 146.92 \\
\cline { 2 - 9 } & $\mathrm{NS}$ & $\mathrm{ML}$ & 6.81 & -0.068 & 31.38 & 0.336 & 0.092 & 8.45 & 154.96 \\
\cline { 2 - 10 } & & $\mathrm{B}-\mathrm{MCMC}$ & 6.71 & 0.058 & 31.60 & 0.295 & -0.298 & 3.03 & 152.12 \\
\hline
\end{tabular}

\section{- Wales}

Figure 5.7a presents the comparison of the observed and the projected AMDR fitted by stationary GEV model and their PDF and CDF curves. It can be observed that all ensemble members of the UKCP18 are generally smaller than the corresponding observed AMDR while member 5 (M5) is the series closest to the observation, therefore the M5 series is selected to represent the climate projection data for comparison with the observation. Unlike that in the England case, the deviation of projected members from their mean values does not maintain unchanged from the observed time series (i.e., not parallel among the GEV modelled AMDR curves). Figure $5.7 \mathrm{~b}$ depicts the return levels calculated under both stationary and nonstationary assumptions whose corresponding models are estimated by ML and B-MCMC methods, shown at two specific years 1990 and 2000. In general, the temporal change of the observed AMDR (denoted by the red circles) is significant during the 20 -year period because in the first 10 years (1981-1990), all events are lower than $45 \mathrm{~mm}$ while the number of events whose rainfall is higher than $45 \mathrm{~mm}$ dramatically increased during last 10 years (1991-2000), i.e., 4 events. Not only are the events of higher rainfall, but the number of lower rainfall (e.g., AMDR is lower than $30 \mathrm{~mm}$ ) also significantly increased, which caused an increasing deviation between the two time series (that can be reflected by the scale parameter $\sigma$ to some degree). 

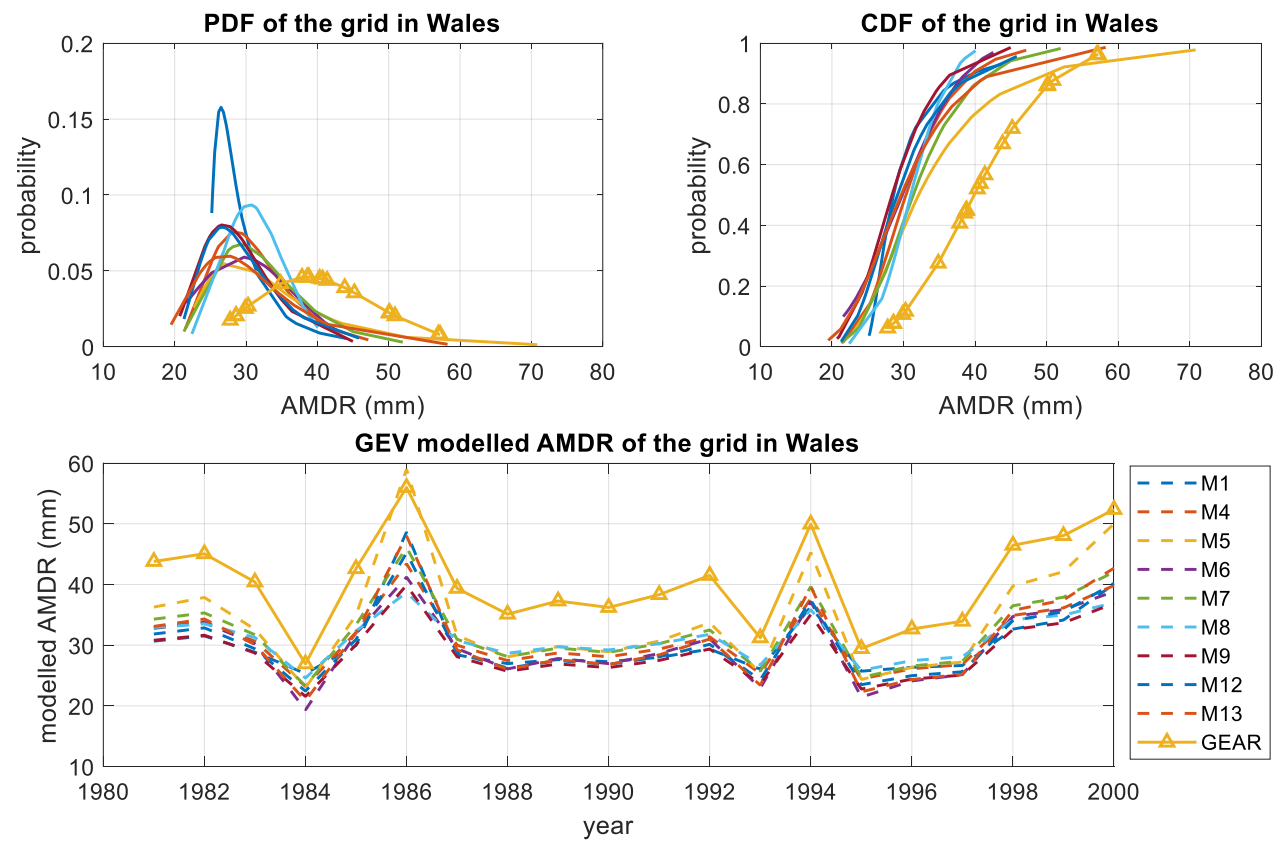

(a)

S-GEAR/UKCP18

(ML)

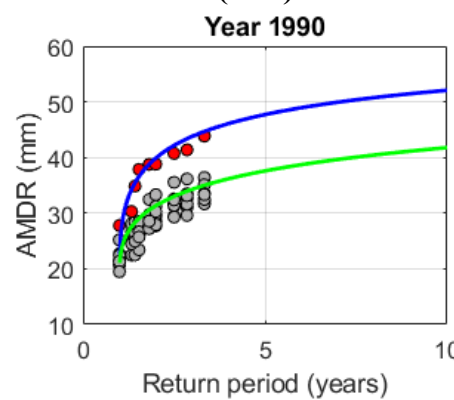

Year 2000

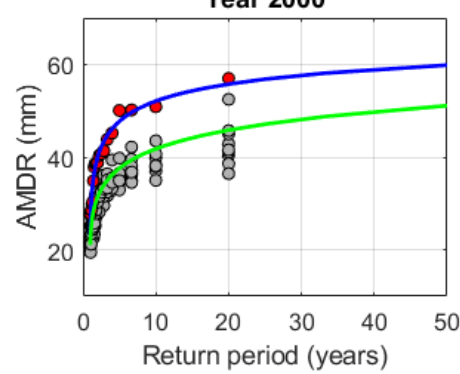

NS-GEAR/UKCP18

(ML)

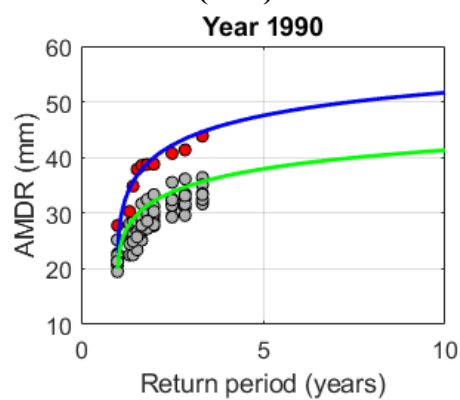

Year 2000

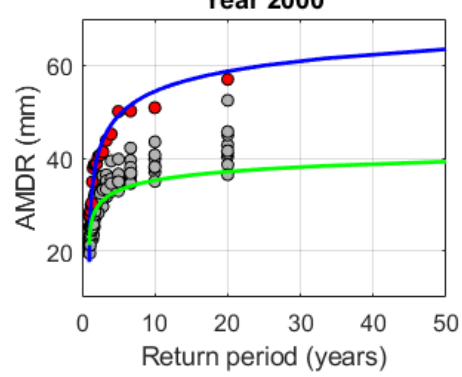

(b)
NS-GEAR/UKCP18

(B-MCMC)

Year 1900

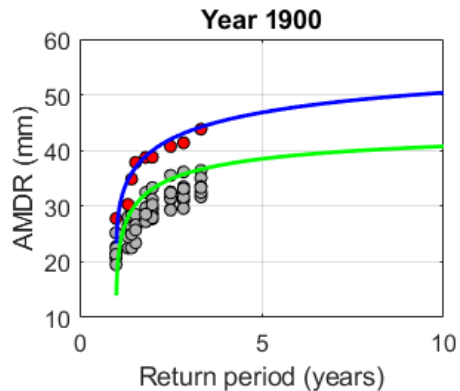

Year 2000

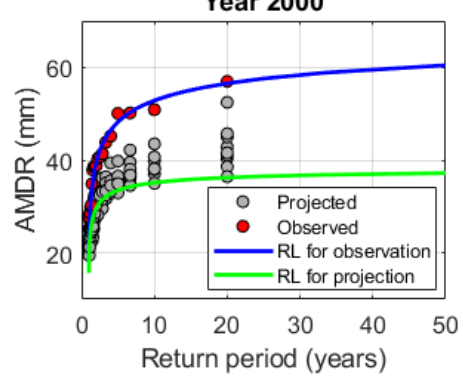

Figure 5.7 Comparison between stationary GEV of observed and climate projected AMDR in the study area of

Wales (a); and comparison of stationary (S) and nonstationary (NS) return levels of observed (GEAR) and climate projected (UKCP18) AMDR at the specific years 1990 and 2000 (b). 
Table 5.5 presents the estimated parameters of both stationary and nonstationary GEV models which are used to fit both the observed time series of AMDR and the projected one. The best model for the observed AMDR is the nonstationary model estimated by the B-MCMC method and the scale parameter is estimated to be increasing with time while the location parameter is decreased. These estimation results are consistent with the analysis above in Figure 5.7b, i.e., the most frequent rainfall is decreasing while the occurrence probability of extreme events is increasing. Like the observation time series, the location parameter of the projected time series also decreases but the reduction ratio (i.e., $\mu_{1}$ ) is greater than the observation one. However, the change of $\sigma$ of two best selected models is not in the same direction. The nonstationary GEV model fitting climate projection data has a decreasing $\sigma$ which indicates a gradual decrease on the deviation from the mean value.

Table 5.5 Estimated parameters of stationary (S) and nonstationary (NS) GEV models of both observed and projected AMDR in the study area of Wales.

\begin{tabular}{|c|c|c|c|c|c|c|c|c|c|}
\hline AMDR & $\begin{array}{c}\text { GEV } \\
\text { model }\end{array}$ & Estimator & $\sigma_{0}(\sigma)$ & $\sigma_{1}$ & $\mu_{0}(\mu)$ & $\mu_{1}$ & $\xi$ & $\begin{array}{c}\text { RMSE } \\
(\mathrm{mm})\end{array}$ & AIC \\
\hline \multirow{3}{*}{$\begin{array}{l}\text { Observed } \\
\text { (GEAR) }\end{array}$} & $\mathrm{S}$ & ML & $(8.16)$ & - & $(36.99)$ & - & -0.181 & 3.15 & 150.90 \\
\hline & \multirow{2}{*}{ NS } & ML & 5.57 & 0.246 & 38.54 & -0.139 & -0.204 & 1.98 & 156.73 \\
\hline & & B-MCMC & 5.57 & 0.223 & 38.58 & -0.140 & -0.248 & 1.56 & 156.30 \\
\hline \multirow{3}{*}{$\begin{array}{c}\text { Projected } \\
\text { (UKCP18) }\end{array}$} & $\mathrm{S}$ & ML & $(5.47)$ & - & $(29.33)$ & - & 0.010 & 3.89 & 138.84 \\
\hline & \multirow{2}{*}{ NS } & $\mathrm{ML}$ & 8.18 & -0.228 & 32.44 & -0.242 & -0.148 & 4.36 & 143.45 \\
\hline & & B-MCMC & 9.18 & -0.201 & 33.17 & -0.284 & -0.482 & 2.68 & 128.11 \\
\hline
\end{tabular}

\subsection{Summary and remarks}

In this chapter, three study areas with a size of $0.4^{\circ} \times 3.15^{\circ}$ located in south-middle England, middle Scotland and Wales are selected. An observed and two climate projected annual maximum daily rainfall (AMDR) time series are extracted from $1 \times 1 \mathrm{~km}^{2}$ grid-based GEAR dataset, $0.4^{\circ} \times 3.15^{\circ}$ grid-based ERA20CM dataset and $2.1 \times 2.1 \mathrm{~km}^{2}$ grid-based UKCP18 dataset, respectively. Both stationary and nonstationary generalised extreme value (GEV) models estimated by maximum likelihood (ML) and Bayesian Markov-Chain Monte-Carlo (B- 
MCMC) methods are applied to simulate the AMDR at different return periods and the following conclusion can be drawn from this study:

1) GEV distribution is an appropriate choice for fitting observational AMDR in GB:

- nonstationary GEV model estimated by B-MCMC is usually the best choice for simulating the AMDR in south-middle England, middle Scotland and Wales over both the periods of 1900-2010 and 1981-2010 for capturing the temporal changes of extreme rainfall.

- During 1900 and 2010, the observed changes of AMDR in south-middle England are insignificant but the stationary GEV model underestimates the risk of extreme rainfall at higher return levels, therefore, the nonstationary GEV model estimated by the B-MCMC method is selected as the best one. It shows a small increase in both the most frequent AMDR and the occurrence probability of extreme rainfall. During the short period, the most frequent AMDR is also observed decreasing while the occurrence probability of extreme rainfall is increased.

- During 1900 and 2010, the observed changes of AMDR in middle Scotland are significant and show both increase in the most frequent AMDR and the occurrence probability of extreme rainfall.

- During 1900 and 2010, the observed changes of AMDR in Wales are significant and show both increase in the most frequent AMDR and the occurrence probability of extreme rainfall; during the short period, the most frequent AMDR is observed decreasing while the occurrence probability of extreme rainfall is increased.

- However, the nonstationary GEV model for simulating the ERA20CM extreme rainfall series in three study areas degenerates to the stationary GEV model.

2) In south-middle England, GEV models fitted to the ERA20CM data are able to capture the location parameter $\mu$ but underestimate the scale parameter $\sigma$ of the long period AMDR series while the UKCP18 based model overestimates both parameters but has a better estimation on $\sigma$. In other words, the ERA20CM based model outperforms in simulating the most frequent AMDR while the UKCP18 based model performs better in capturing the time-varying difference between extremes and average. 
3) In middle Scotland, the ERA20CM based model underestimates both parameters, i.e., the scale parameter $\sigma$ is the only half value of $\sigma$ of the observed model while the location parameter $\mu$ is 10 smaller than the observed one over the long period. In other words, climate projection greatly underestimates the most frequent extreme rainfall and cannot capture the difference between more extreme events and such most frequent levels.

4) In middle Wales, the ERA20CM based model underestimates both parameters over the long period, i.e., the scale parameter $\sigma$ is only around half value of $\sigma$ of the observed model while the location parameter $\mu$ is around 15 smaller than the observed model. The UKCP18 based model also underestimates both parameters but obtains a consistent decrease trend of $\mu$ with the observed AMDR. However, in general, the UKCP18 based model performs than the ERA20CM based model.

These findings point out that although the climate projections have been remarkably improved with a finer resolution e.g., the UKCP18 data have a higher spatial resolution hence can simulate better than the ERA20CM, the improvements are not enough nor effective due to many aspects such as technology limitations, knowledge level of nature, etc, especially in terms of revealing extreme events. Many climate projections from climate models often suffer from: 1) being unable to simulate extremes albeit being good at simulating the average; 2) being unable to simulate the time-varying change in climate extreme. Therefore, the study in this chapter is carried out to quantify how good the climate projections can be in representing the occurrences and temporal variation of the extremes by using a nonstationary probability framework. The gap in the ability of climate projections to capture the observations is described by the time-varying GEV parameters, which offers further insight into the utility of climate projection datasets when extreme quantities instead of the averages are at stake in applications. 


\section{Chapter 6 Nonstationary Multivariate}

\section{Framework: A Case Study of Compound Flooding Simulation in Ho-Chi-Minh City, Vietnam}

So far, the previous chapters have focused on the modelling framework for quantifying spatiotemporal variation of univariate hydroclimatic extremes, this chapter extends the analysis to multivariate and proposes a nonstationary multivariate framework for quantifying the timevarying joint probability of two meteorological and oceanographic drivers which leads to a compound flood. This chapter uses Ho Chi Minh City (HCMC), one of the most vulnerable coastal cities in southeast Asia to compound floods, as a case study to illustrate the application of the proposed multivariate framework. The proposed nonstationary multivariate analysis framework considers four combinations of assumptions of marginals (maximum rainfall and skew surge) and copula to be either stationary or nonstationary and analyses the variation of the worst compound floods in both wet and dry seasons.

\subsection{Introduction}

Flooding is widely regarded as one of the most dangerous natural hazards (Jonkman, 2005). It often arises from various sources such as extreme rainfall, storm surge, high sea level, large river discharge either individually or in combination, (Bevacqua et al., 2020; Hendry et al., 2019). However, the concurrence or close succession of these different source mechanisms can lead to compound flooding, resulting in greater damage than from separate events caused by 
the individual mechanism (AghaKouchak et al., 2020; Hendry et al., 2019). This is further exemplified by the occurrence of several recent events where inland floods are associated with hydrologic drivers (e.g., rainfall, river discharge) combined with oceanographic drivers (e.g., tides, storm surges, waves). Examples of this include the compound floods on the North Carolina Coast of USA (Gori et al., 2020); in the Shoalhaven estuary, Australia in June 2016 (Kumbier et al., 2018); the Noorderzijlvest, the Netherlands in 2015 (van den Hurk et al., 2015); and in Ravenna, Italy in 2015 (Bevacqua et al., 2017). To understand the characteristics of such high-impact compound events, one effective and commonly used approach is the multivariate analysis, which can consider the interdependence, interaction and associations among different drivers, and thereby better estimate flood occurrence probability. As one of the most popular approaches in various multivariate analysis methods, the copula is widely used for modelling the dependence structure of two or more random variables since Sklar (1959) proposed the concept that is to quantify the link (i.e., the joint probability) between the marginal distributions of variables. The advantages of using copula are that it allows the dependence among multiple variables to be modelled and also allows the marginals and their correlation separately (Embrechts et al., 2001). Nowadays, many parametric copula families are available (e.g., Elliptical copulas, Gaussian copulas, Archimedean copulas) and have been widely applied in many areas such as quantitative finance (D'Amico and Petroni, 2018; Dias and Embrechts, 2004), medicine (Emura et al., 2020), signal processing (Jovanovic et al., 2018; Parchami et al., 2020) and climate research (Jhong and Tung, 2018; Won et al., 2020).

In hydroclimatic sciences, notably, many studies have already employed copulas to model the dependence structure among hydrological variables for evaluating the compound events, e.g., Zhu et al. (2019); Renard and Lang (2007); Zhang and Singh (2006); Favre et al. (2004); Salvadori and De Michele (2004). However, global warming has led to significant changes in regional climate (Ricke et al., 2010) which can cause variability in climate variables such as temperature, precipitation, sea level, snowpack, drought, and heatwave. When considering compound events, not only these climate variables themselves can vary with time, but also their correlation can be nonstationary, e.g., the interaction becomes more significant or less due to climate change (Villalobos-Herrera et al., 2021; Zscheischler et al., 2019). Since many water 
infrastructures have a typical design life of several decades, their sustainability is challenged in a nonstationary climate, and an overhaul of the current storm design determination may be needed as climate change continues and compound processes are altered (Moftakhari et al., 2017). Therefore, involving the perspective of non-stationarity is essential. The last few decades have also witnessed a great deal of interest and efforts in univariate nonstationary modelling in hydrological risk assessment, e.g., Cancelliere (2017); Tramblay et al. (2013). Some recent progress on multivariate nonstationary studies has been reviewed here. Chebana et al. (2013) first proposed to assume a time-varying dependence structure between multivariate hydrological variables to estimate their joint probability. Kwon and Lall (2016) quantified the time-varying joint probabilities of the severity and duration of drought in California by modelling the nonstationary marginal distributions of these two variables, which were linked by a stationary Gumbel copula. Sarhadi et al. (2018) quantified the temporal changes in the joint probability of warm and dry conditions happening in an individual location and simultaneously in multiple locations by assuming time-varying parameters of copulas. Feng et al. (2020) investigated flood risk under nonstationary conditions arising from climate change when floods occur simultaneously in the Huai River and Hong River of China. They assumed nonstationary marginal distributions of flood magnitudes of the two rivers and applied dynamic copulas to calculate the joint probability. However, these latest studies focused on compound situations driven only by the same or similar type of variables such as flood volume and peak which are certainly intercorrelated but less meaningful for contributing to compound events which can be driven by different variables. However, the quantification of the temporal changes on joint probability of different variables leading to compound floods in the view of nonstationarity, especially at the level of extreme, have yet been fully studied.

Therefore, in this chapter, based on the nonstationary quantitative framework proposed in Part I for modelling univariate changes, I extend this framework to be multivariate that aims to develop a more feasible framework to estimate the joint probability of different variables in the context of different situations/assumptions, e.g., both the marginal distributions of variables and their correlation structure can be either stationary or nonstationary. To demonstrate the applicability of the framework, a case study is provided to estimate the impact of compound 
floods driven by inland rainfall and skew surge in Ho Chi Minh City (HCMC), Vietnam. As the characteristics of flood in HCMC have a strong seasonal dependency, monthly maximum series of daily rainfall and skew surge are used and two months for representing dry and wet seasons are especially analysed where the flood inundation maps are generated by a hydrodynamic model (TELEMAC-2D). This nonstationary compound flood modelling system based on the proposed framework is expected to be used by the National Centre for HydroMeteorological Forecasting (NCHMF) of Vietnam for prediction at the national level.

The remainder of this paper is organised as follows: Section 6.2 describes the framework of nonstationary copula; Section 6.3 explains the case study, HCMC, the data processing and the hydrodynamic modelling. The results from the application of the framework to the case study are presented in Section 6.4. Concluding remarks on the framework and the case study are given in Section 6.5.

\subsection{A nonstationary framework of multivariate probability distribution analysis}

Figure 6.1 presents the framework developed to analyse compound floods driven by both the hydrometeorological driver (e.g., monthly maximum rainfall, MMR) and oceanographic driver (e.g., monthly maximum skew surge, MMS) in view of non-stationarity, in turn, linked to climate change. It can be described by the four main steps which are further elaborated respectively in the following subsections. 


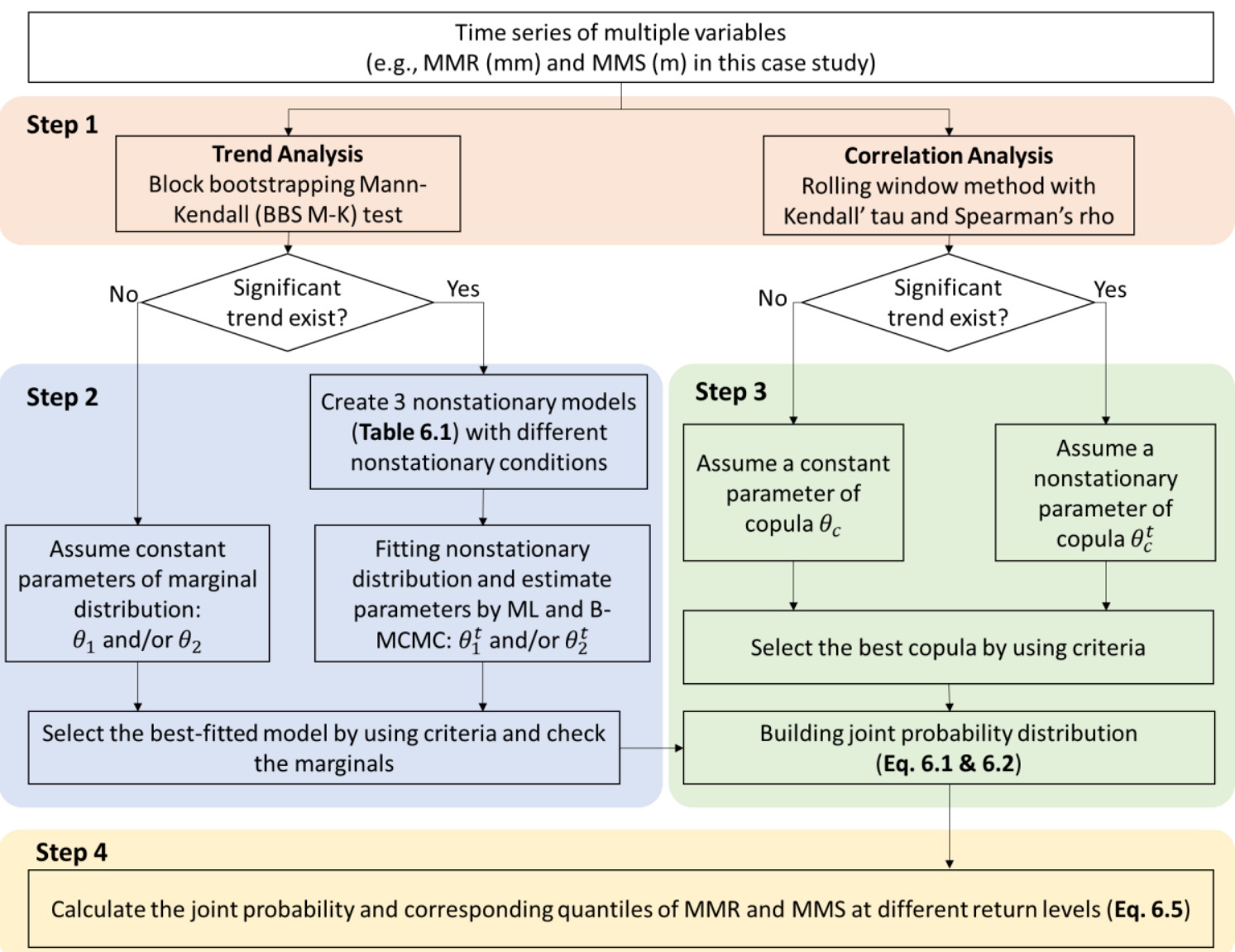

Figure 6.1 Nonstationary framework of multivariate probability distribution analysis. MMR is the monthly maximum rainfall; MMS is the monthly maximum skew surge.

\subsubsection{Trend and correlation analysis}

This step aims to detect whether the values of each flood driver, and the correlation structure between values of multiple drivers change with other covariates. As the possible temporal nonstationary nature of the driver is the main objective of the study, time is chosen as the covariate. This will be the basis for the parameterisation of the copula to be proposed. Here, Block Bootstrapping Mann-Kendall (BBS-MK) test (Kundzewicz and Robson, 2004; Önöz and Bayazit, 2012) is employed for detecting monotonic trends in series of data at the significance level of 0.05. The details of the BBS-MK test are described in Section 4.2.1. 
Parallel to this, to test the correlation between the series of data and its changes over time, I employ Kendall's tau and Spearman correlation analysis associated with a Rolling window method, which is both widely used (de Winter et al., 2016; Zar, 2005). The correlation coefficients, i.e., $\tau$ and $\rho$, respectively, indicate the possible positive or negative correlation between the time series, while the corresponding $p$-values are compared with the critical values at the significance level of 0.05 to decide whether to reject the null hypothesis that no correlation exists. The Rolling window method is employed to use a variety of predefined widths of the window and move forward to the end of the data (Inoue et al., 2017), which is carried out following the three steps:

i. Select the width of the rolling window $m$ which is the number of consecutive series of data per window. The selection of width is usually based on the total number $S$ and periodicity of the data $(m<S)$. As the result can be sensitive to the selection of the block length, several different lengths are tested (i.e., 10, 20 and 30 years) so that the consistency of the results are ensured.

ii. Set the number of increments between the successive rolling window, i.e., the moving step, as 1 year. In other words, I partition the data into $N=S-m+1$ subsamples and the first rolling window covers the data from 1 to $m$ and the second covers 2 to $m+1$ and so on.

iii. For each rolling window, the correlation is tested by two tests and calculate the correlation coefficients and the $p$-value at the significance level of 0.05 .

\subsubsection{Model marginal distributions of the series of data}

In this step, several widely used types of probability distributions are selected as the marginal distribution candidates to fit each series, which includes Generalised Extreme Value (GEV) distribution, Generalised Pareto distribution, Gamma distribution, Lognormal distribution and Exponential distribution. Following the outcomes from Section 6.2.1 (i.e., Step 1 shown in Figure 6.1) Two assumptions can be made for the marginal distribution: of stationarity, which means that the parameters of the distribution remain constant and independent and can be estimated by the Maximum Likelihood (ML) method; and of non-stationarity, which means 
that several parameters of the distribution are assumed to be changing over time and parameters can be estimated by both the ML and the Bayesian Markov-Chain Monte-Carlo (B-MCMC) methods. A stationary and three non-stationary assumptions of these distributions are presented in Table 6.1. For the time series whose trend is determined to be insignificant at the significance level of 0.05 , only stationary distributions are applied, while when the trend is significant, both stationary and non-stationary distributions are applied. The best-fitted distribution is finally selected by evaluating two criteria: Akaike's information criterion (AIC) and Bayesian information criterion (BIC); and the minimum values indicate the better performance of the model.

Table 6.1 Stationary (S) and nonstationary (NS) candidate distributions for time series. For the explanation on symbols please see Section 6.2.2.

\begin{tabular}{|c|c|c|c|}
\hline Distribution & $\begin{array}{l}\text { Mo } \\
\text { del }\end{array}$ & Description & Parameters $(\boldsymbol{\theta})$ \\
\hline \multirow{4}{*}{$\begin{array}{l}\text { Generalised } \\
\text { extreme } \\
\text { value } \\
\text { distribution }\end{array}$} & $\mathrm{S}$ & $\begin{array}{l}F(x ; \sigma, \mu, \xi) \\
=\left\{\begin{array}{c}\exp \left[-\left(1+\xi\left(\frac{x-\mu}{\sigma}\right)\right)^{-1 / \xi}\right], \xi \neq 0 \\
\exp \left[-\exp \left(\frac{x-\mu}{\sigma}\right)\right], \xi=0\end{array}\right. \\
\text { where } 1+\xi(x-\mu) / \sigma>0,-\infty< \\
\mu<\infty, \sigma>0 \text { and }-\infty<\xi<\infty .\end{array}$ & $\begin{array}{c}\boldsymbol{\theta}=\{\sigma, \mu, \xi\} \\
\text { where } \\
\sigma, \mu, \xi \text { are all constant. }\end{array}$ \\
\hline & NS1 & $F_{t}\left(x ; \sigma, \mu_{t}, \xi\right)$ & $\begin{array}{c}\boldsymbol{\theta}_{\boldsymbol{t}}=\left\{\mu_{0}, \mu_{1}, \sigma, \xi\right\} \\
\text { where } \\
\mu_{t}=\mu_{0}+\mu_{1} \times t \\
\sigma, \xi \text { are constant }\end{array}$ \\
\hline & NS2 & $F_{t}\left(x ; \sigma_{t}, \mu_{t}, \xi\right)$ & $\begin{array}{c}\boldsymbol{\theta}_{\boldsymbol{t}}=\left\{\mu_{0}, \mu_{1}, \sigma_{0}, \sigma_{1}, \xi\right\} \\
\text { where } \\
\mu_{t}=\mu_{0}+\mu_{1} \times t \\
\sigma_{t}=\sigma_{0}+\sigma_{1} \times t \\
\xi \text { is constant }\end{array}$ \\
\hline & NS3 & $F_{t}\left(x ; \sigma_{t}, \mu_{t}, \xi\right)$ & $\begin{array}{c}\boldsymbol{\theta}_{\boldsymbol{t}}=\left\{\mu_{0}, \mu_{1}, \sigma_{0}, \sigma_{1}, \xi\right\} \\
\text { where } \\
\mu_{t}=\mu_{0}+\mu_{1} \times t \\
\sigma_{t}=\exp \left(\sigma_{0}+\sigma_{1} \times t\right) \\
\xi \text { is constant }\end{array}$ \\
\hline
\end{tabular}




\begin{tabular}{|c|c|c|c|}
\hline \multirow{4}{*}{$\begin{array}{l}\text { Generalised } \\
\text { Pareto } \\
\text { distribution }\end{array}$} & S & $\begin{array}{l}F(x ; \sigma, \mu, \xi) \\
=\left\{\begin{array}{c}1-\left(1+\xi\left(\frac{x-\mu}{\sigma}\right)\right)^{-1 / \xi}, \xi \neq 0 \\
1-\exp \left(-\frac{x-\mu}{\sigma}\right), \xi=0\end{array}\right. \\
\text { where } x \geq \mu \text { when } \xi \geq 0 \text { and } \mu \leq \\
\quad x \leq \mu-\sigma / \xi \text { when } \xi<0 .\end{array}$ & $\begin{array}{c}\boldsymbol{\theta}=\{\sigma, \mu, \xi\} \\
\text { where } \\
\sigma, \mu, \xi \text { are all constant }\end{array}$ \\
\hline & NS1 & $F_{t}\left(x ; \sigma, \mu_{t}, \xi\right)$ & $\begin{array}{c}\boldsymbol{\theta}_{\boldsymbol{t}}=\left\{\mu_{0}, \mu_{1}, \sigma, \xi\right\} \\
\quad \text { where } \\
\mu_{t}=\mu_{0}+\mu_{1} \times t \\
\sigma, \xi \text { are constant }\end{array}$ \\
\hline & NS2 & $F_{t}\left(x ; \sigma_{t}, \mu_{t}, \xi\right)$ & $\begin{array}{c}\boldsymbol{\theta}_{\boldsymbol{t}}=\left\{\mu_{0}, \mu_{1}, \sigma_{0}, \sigma_{1}, \xi\right\} \\
\text { where } \\
\mu_{t}=\mu_{0}+\mu_{1} \times t \\
\sigma_{t}=\sigma_{0}+\sigma_{1} \times t \\
\xi \text { is constant }\end{array}$ \\
\hline & NS3 & $F_{t}\left(x ; \sigma_{t}, \mu_{t}, \xi\right)$ & $\begin{array}{c}\boldsymbol{\theta}_{\boldsymbol{t}}=\left\{\mu_{0}, \mu_{1}, \sigma_{0}, \sigma_{1}, \xi\right\} \\
\text { where } \\
\mu_{t}=\mu_{0}+\mu_{1} \times t \\
\sigma_{t}=\exp \left(\sigma_{0}+\sigma_{1} \times t\right) \\
\quad \xi \text { is constant }\end{array}$ \\
\hline \multirow{3}{*}{$\begin{array}{c}\text { Gamma } \\
\text { distribution }\end{array}$} & $\mathrm{S}$ & $\begin{array}{c}F(x ; \sigma, \xi)=\frac{1}{\Gamma(\xi)} \gamma\left(k, \frac{x}{\sigma}\right) \\
\text { Where } x>0, \sigma>0, \xi>0 .\end{array}$ & $\begin{array}{c}\boldsymbol{\theta}=\{\sigma, \xi\} \\
\text { where } \\
\sigma, \xi \text { are all constant }\end{array}$ \\
\hline & NS1 & $F_{t}\left(x ; \sigma, \xi_{t}\right)$ & $\begin{array}{c}\boldsymbol{\theta}_{\boldsymbol{t}}=\left\{\xi_{0}, \xi_{1}, \sigma\right\} \\
\text { where } \\
\xi_{t}=\xi_{0}+\xi_{1} \times t \\
\sigma \text { is constant }\end{array}$ \\
\hline & NS2 & $F_{t}\left(x ; \sigma_{t}, \xi_{t}\right)$ & $\begin{aligned} \boldsymbol{\theta}_{\boldsymbol{t}}= & \left\{\xi_{0}, \xi_{1}, \sigma_{0}, \sigma_{1}\right\} \\
& \text { where } \\
\xi_{t}= & \xi_{0}+\xi_{1} \times t \\
\sigma_{t}= & \sigma_{0}+\sigma_{1} \times t\end{aligned}$ \\
\hline \multirow{3}{*}{$\begin{array}{l}\text { Lognormal } \\
\text { distribution }\end{array}$} & $S$ & $\begin{array}{c}F(x ; \sigma, \mu)=\varnothing\left(\frac{\ln x-\mu}{\sigma}\right) \\
\text { Where } \emptyset \text { is the cumulative } \\
\text { distribution function of the standard } \\
\text { normal distribution. }\end{array}$ & $\begin{array}{c}\boldsymbol{\theta}=\{\sigma, \mu\} \\
\text { where } \\
\sigma, \mu \text { are all constant }\end{array}$ \\
\hline & NS1 & $F_{t}\left(x ; \sigma, \mu_{t}\right)$ & $\begin{array}{c}\boldsymbol{\theta}_{\boldsymbol{t}}=\left\{\mu_{0}, \mu_{1}, \sigma\right\} \\
\text { where } \\
\mu_{t}=\mu_{0}+\mu_{1} \times t \\
\sigma \text { are constant }\end{array}$ \\
\hline & NS2 & $F_{t}\left(x ; \sigma_{t}, \mu_{t}\right)$ & $\begin{array}{c}\boldsymbol{\theta}_{\boldsymbol{t}}=\left\{\mu_{0}, \mu_{1}, \sigma_{0}, \sigma_{1}\right\} \\
\text { where }\end{array}$ \\
\hline
\end{tabular}




\begin{tabular}{|c|c|c|c|}
\hline & & & $\begin{aligned} \mu_{t} & =\mu_{0}+\mu_{1} \times t \\
\sigma_{t} & =\sigma_{0}+\sigma_{1} \times t\end{aligned}$ \\
\hline & NS3 & $F_{t}\left(x ; \sigma_{t}, \mu_{t}\right)$ & $\begin{array}{c}\boldsymbol{\theta}_{\boldsymbol{t}}=\left\{\mu_{0}, \mu_{1}, \sigma_{0}, \sigma_{1}, \xi\right\} \\
\text { where } \\
\mu_{t}=\mu_{0}+\mu_{1} \times t \\
\sigma_{t}=\exp \left(\sigma_{0}+\sigma_{1} \times t\right)\end{array}$ \\
\hline \multirow{3}{*}{$\begin{array}{l}\text { Exponential } \\
\text { distribution }\end{array}$} & S & $\begin{array}{l}\qquad F(x ; \sigma)=\left\{\begin{array}{c}\frac{1}{\sigma} \exp \left(-\frac{x}{\sigma}\right), x>0 \\
0, x<0\end{array}\right. \\
\text { Where the scale parameter } \sigma=1 / \lambda \\
\text { and } \lambda>0 \text { is the rate parameter of the } \\
\text { exponential distribution. }\end{array}$ & $\begin{array}{c}\boldsymbol{\theta}=\{\sigma\} \\
\text { where } \\
\sigma \text { is constant }\end{array}$ \\
\hline & NS2 & $F_{t}\left(x ; \sigma_{t}\right)$ & $\begin{array}{c}\boldsymbol{\theta}_{\boldsymbol{t}}=\left\{\sigma_{0}, \sigma_{1}\right\} \\
\\
\text { where } \\
\sigma_{t}=\sigma_{0}+\sigma_{1} \times t\end{array}$ \\
\hline & NS3 & $F_{t}\left(x ; \sigma_{t}\right)$ & $\begin{array}{c}\boldsymbol{\theta}_{\boldsymbol{t}}=\left\{\sigma_{0}, \sigma_{1}\right\} \\
\text { where } \\
\sigma_{t}=\exp \left(\sigma_{0}+\sigma_{1} \times t\right)\end{array}$ \\
\hline
\end{tabular}

Noted that $\mu, \sigma$ and $\xi$ indicate location, scale and shape parameter of distribution respectively; $\boldsymbol{\theta}$ and $\boldsymbol{\theta}_{\boldsymbol{t}}$ are symbols to indicate the parameters for each model needing to be estimated and the subscript $t$ is used for indicating the nonstationary model; $\mathrm{S}$ is short for "stationarity" case and

NS1, NS2 and NS3 indicate three "non-stationarity" cases.

As previously mentioned, there are several types of candidate distribution. Without losing generality, GEV distribution is used in this example to demonstrate the process which is also followed for other types of distribution. If the best-fitted distribution model of the time series is a GEV distribution, both stationary and nonstationary distributions can be developed following the methods described in Sections 4.2.2 and 4.2.3. For the stationary model (S), there are three constant parameters $\mu, \sigma$ and $\xi$ which are estimated by the ML method.

\subsubsection{Build copulas and calculate the joint probability}

Let $J$ denote the joint cumulative distribution function of the two series of data, and $C$ denote the copula function parameterized by $\theta_{C}$. Then, the basic joint probability can be calculated by:

$$
J\left(x_{S}, x_{R} \mid \theta_{C}\right)=C\left(F_{1}\left(x_{1} \mid \boldsymbol{\theta}_{1}\right), F_{2}\left(x_{2} \mid \boldsymbol{\theta}_{2}\right) \mid \theta_{C}\right)=C\left(u, v \mid \theta_{C}\right)
$$

where $F_{1}$ and $F_{2}$ indicate the marginal cumulative probability function of the two series of data $x_{1}$ (in the case study, MMS) and $x_{2}(\mathrm{MMR})$ with their estimated parameters $\boldsymbol{\theta}_{1}$ and $\boldsymbol{\theta}_{2}$, 
respectively and $\theta_{C}$ indicates the set of parameters of the copula. $u$ and $v$ are the marginal probabilities of $F_{1}$ and $F_{2}$ in the unit hypercube with uniform marginal distributions $U(0,1)$. According to the trend analysis of the individual time series and their mutual correlation structure, four contexts are relevant in this framework:

- Both marginal distributions $(\boldsymbol{\theta})$ are stationary, and the correlation structure $\left(\theta_{C}\right)$ is stationary.

- Both marginal distributions $(\boldsymbol{\theta})$ are stationary, while the correlation structure $\left(\theta_{C}^{t}\right)$ is nonstationary.

- At least one of the marginal distributions $\left(\boldsymbol{\theta}_{\boldsymbol{t}}\right)$ is nonstationary, while the correlation structure $\left(\theta_{C}\right)$ is stationary.

- At least one of the marginal distributions $\left(\boldsymbol{\theta}_{\boldsymbol{t}}\right)$ is nonstationary, while the correlation structure $\left(\theta_{C}\right)$ is nonstationary.

In this framework, several widely-used one-parameter copulas are selected as the candidates to characterise the dependence structure between two series of data, namely, Gaussian, Clayton, Frank, Gumbel, Joe, Plackett and Raftery copulas whose parameter $\theta_{C}$ is estimated by using both the local optimization method and MCMC approach and processed by using the MvCAT toolbox (Sadegh et al., 2017). However, if there is no significant correlation identified, i.e., the two variables are independent, I also involve an independent copula which is simply reduced to the form where the joint probability is calculated by the probability function of one variable multiplied by the other.

For the nonstationary copula whose parameter varies over time, I assume that the copula is controlled by $\theta_{C}^{t}$ with two hyper-parameters $\theta_{C 0}$ and $\theta_{C 1}$ and the joint cumulative distribution can be written as:

$$
\begin{gathered}
J_{t}\left(x_{1 t}, x_{2 t} \mid \theta_{C}^{t}\right)=C\left(F_{1 t}\left(x_{1 t} \mid \boldsymbol{\theta}_{1 t}\right), F_{2 t}\left(x_{2(t)} \mid \boldsymbol{\theta}_{2 t}\right) \mid \theta_{C}^{t}\right)=C\left(u_{t}, v_{t} \mid \theta_{C}^{t}\right) \\
\theta_{C}^{t}=\theta_{C 0}+\theta_{C 1} \times t
\end{gathered}
$$

where $\boldsymbol{\theta}_{1 t}$ and $\boldsymbol{\theta}_{2 t}$ indicate the time-varying parameters of the two marginal distributions shown in Table 6.1 and $u_{t}, v_{t}$ are the nonstationary marginal probabilities converting in the 
uniform $\mathrm{U}[0,1]$. These parameters can also be estimated by the B-MCMC method and the posterior joint distribution can be calculated as (Ausin and Lopes, 2010):

$$
p\left(\varnothing \mid x_{1 t}, x_{2 t}\right) \propto p(\varnothing \mid t) \times \prod_{t=1}^{N} p\left(x_{1 t}, x_{2 t} \mid \varnothing, t\right)
$$

where $p\left(x_{1 t}, x_{2 t} \mid \emptyset, t\right)=c\left(F_{1 t}\left(x_{1 t} \mid \boldsymbol{\theta}_{1 t}\right), F_{2 t}\left(x_{2(t)} \mid \boldsymbol{\theta}_{2 t}\right) \mid \theta_{C}^{t}\right) \times f_{1 t}\left(x_{1 t} \mid \boldsymbol{\theta}_{1 t}\right) \times f_{2 t}\left(x_{2 t} \mid \boldsymbol{\theta}_{2 t}\right)$ is the copula density function, $f_{1 t}$ and $f_{2 t}$ are the marginal probability density functions and the parameters of the joint posterior are $\emptyset=\left(\mu_{S 0}, \mu_{S 1}, \sigma_{S 0}, \sigma_{S 1}, \xi_{S}, \mu_{R 0}, \mu_{R 1}, \sigma_{R 0}, \sigma_{R 1}, \xi_{2}, \theta_{C 0}, \theta_{C 1}\right)$. $p(\varnothing \mid t)$ is the prior distribution of the parameters $\emptyset$ and according to the prior knowledge for which I assume a uniform distribution for all parameters, i.e., the parameters of the marginal distributions are assumed to be uniformly distributed around the values estimated by stationary assumption and the copula parameters are assumed to be within the maximum and minimum limits subject to copula types. To reduce the time for running the MCMC algorithm, the nonstationary marginal parameters are firstly estimated by applying the approach in Section 4.2.3 then transform into $u_{t}$ and $v_{t}$. The MCMC algorithm in this step is only used to estimate the copula parameter by:

$$
p\left(\theta_{C}^{t} \mid u_{t}, v_{t}\right) \propto p\left(\theta_{C}^{t} \mid t\right) \times \prod_{t=1}^{N} c\left(u_{t}, v_{t} \mid \theta_{C}^{t}, t\right)
$$

Finally, the best copula is selected by evaluating the goodness of fit measure AIC.

\subsubsection{Generate the quantiles}

The final step of the framework is to calculate the quantiles of the joint exceedance probability that has been defined in the last step. According to the different analysis of the marginal distributions and correlation structure obtained from the previous steps, the stationary context will lead to one quantile with a given probability of $p$, while the nonstationary context will obtain a series of quantiles changing over covariate (i.e., time in the study) for the same given $p$. The quantiles can be expressed as:

$$
Q(p)=\left(x_{1}=F_{1 t}^{-1}\left(u \mid \boldsymbol{\theta}_{1}^{t}\right), x_{2}=F_{2 t}^{-1}\left(v \mid \boldsymbol{\theta}_{2}^{t}\right)\right)
$$


where $p=C_{t}\left(u, v \mid \theta_{C}^{t}\right)$ and $t$ indicates that the parameters or variables are changing over time. If the context is stationary and two variables are independent, $Q(p)$ can be simply calculated by inverting the marginal distributions, i.e., $x_{1}=F_{1}^{-1}\left(u \mid \boldsymbol{\theta}_{1}\right)$ and $x_{2}=F_{2}^{-1}\left(v \mid \boldsymbol{\theta}_{2}\right)$.

There are several approaches to select the cases of combination of marginals (e.g., the variable of $x_{1}$ and $x_{2}$ ) and the most used approach is to get the most likely combination with the highest joint density level (Sadegh et al., 2018; Salvadori et al., 2014). For example, Figure 6.2 illustrates all quantile curves corresponding to the probability from $p=0.01$ to $p=0.99$. The horizontal $\mathrm{x}$-axis and vertical $\mathrm{y}$-axis are two marginal variables where all the combinations of these two variables along the same curve correspond to the same $p$. The most likely scenarios method is to peak up the combination where the joint density of this quantile curve is the highest. In this example, the z-axis of Figure 6.2 indicates the joint density level uniformed to the range of $(0,1)$ and the blue circles indicate the location where the density level is 1 and the combination of MMS and MMR is regarded as the most likely one. The other commonly used approach is to sample all the combinations on the same quantile curve instead of selecting only one combination, however, it means the scenarios selection has stochasticity which requires to be further analysed. In this case study, the most likely scenario approach is applied. 


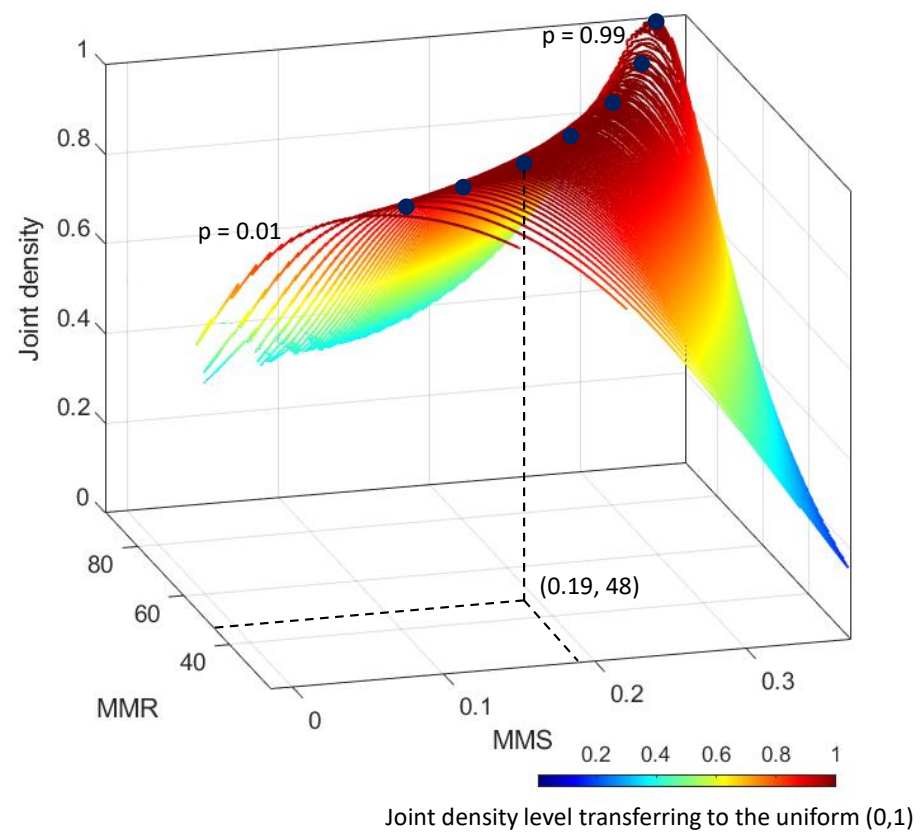

Figure 6.2 Joint density level of quantile curves corresponding to the probability from 0.01 to 0.99

\subsection{Case study}

\subsubsection{Study domain and data processing}

Ho Chi Minh City (HCMC) is located in the downstream reach of the Saigon and Dong Nai rivers (see Figure 6.3). As a major economic centre of Vietnam, it has emerged as one of the fastest-growing cities in Southeast Asia. With its nearly 10 million inhabitants, the city contributes more than 20\% GDP of Vietnam (Hoi, 2020; Kontgis et al., 2014). Yet, due to its geographical location and the ageing infrastructure, the city is vulnerable to the frequent floods resulting from concurrent heavy rainfall and storm surges, commonly known as compound flooding (Binh et al., 2019; Horton et al., 2010; Molenaar et al., 2010; Nguyen et al., 2019). Rising sea levels have been driving the threats of compound flooding to an even higher level with further complications. More worryingly, as around $65 \%$ of the city is below $1.5 \mathrm{~m}$ above the mean sea level (Cao et al., 2021; Scussolini et al., 2017; Vachaud et al., 2019), the inhabitants are affected by flooding with their living conditions deteriorating in the context of high SLR which has increased by $20 \mathrm{~cm}$ over the past 50 years and has been expected to 
continue increasing (Thuc et al., 2016). This study selects HCMC as the case where the drivers of compound floods are analysed by applying the nonstationary framework I developed, taking climate change into account. As discussed in Couasnon et al., (2021), the two main drivers of the flooding in HCMC are intense rainfall, often associated with strong surges, which is also chosen in this study.

Rainfall: Daily rainfall data collected from six rain gauges in the vicinity of the study domain (see Figure 6.3) were obtained from the Southern Regional Hydrometeorological Centre. The data cover a period of 38 years from $01 / 01 / 1980$ to 31/12/2017. The gauged rainfall was then converted to areal rainfall by applying the Thiessen polygon method (Couasnon et al., 2021).

Skew surge: Hourly Sea water level data of the same period at the estuary of HCMC (Vung Tau water level gauge, see Figure 6.3) were also provided by the Southern Regional Hydrometeorological Centre. The skew surge was then calculated by subtracting tidal cycles from the total sea level (Cid et al., 2017; Wu et al., 2018). To avoid the spurious peaks in the residual signal due to minor phase shifts in the tidal predictions, the skew surge rather than storm surge is calculated and used in the study, which is the difference between the highest observed sea level and high tide within a tidal period and finally, the time series of the daily maximum skew surge is generated and more details for processing the data can be checked in Couasnon et al. (2021).

HCMC has a dry season, from December till April, and a wet season, from May to November. As demonstrated by the previous research in Couasnon et al. (2021), understanding the seasonality of each flood driver is important to prevent the over-or underestimation of flood impacts in the area affected by pluvial, fluvial or coastal floods such as HCMC. Therefore, I extracted both monthly maxima from the series of daily areal rainfall (henceforth, MMR) and of daily skew surge (henceforth, MMS) between 1980 and 2017 and estimated the dependence and risk, again, in a seasonal fashion. 


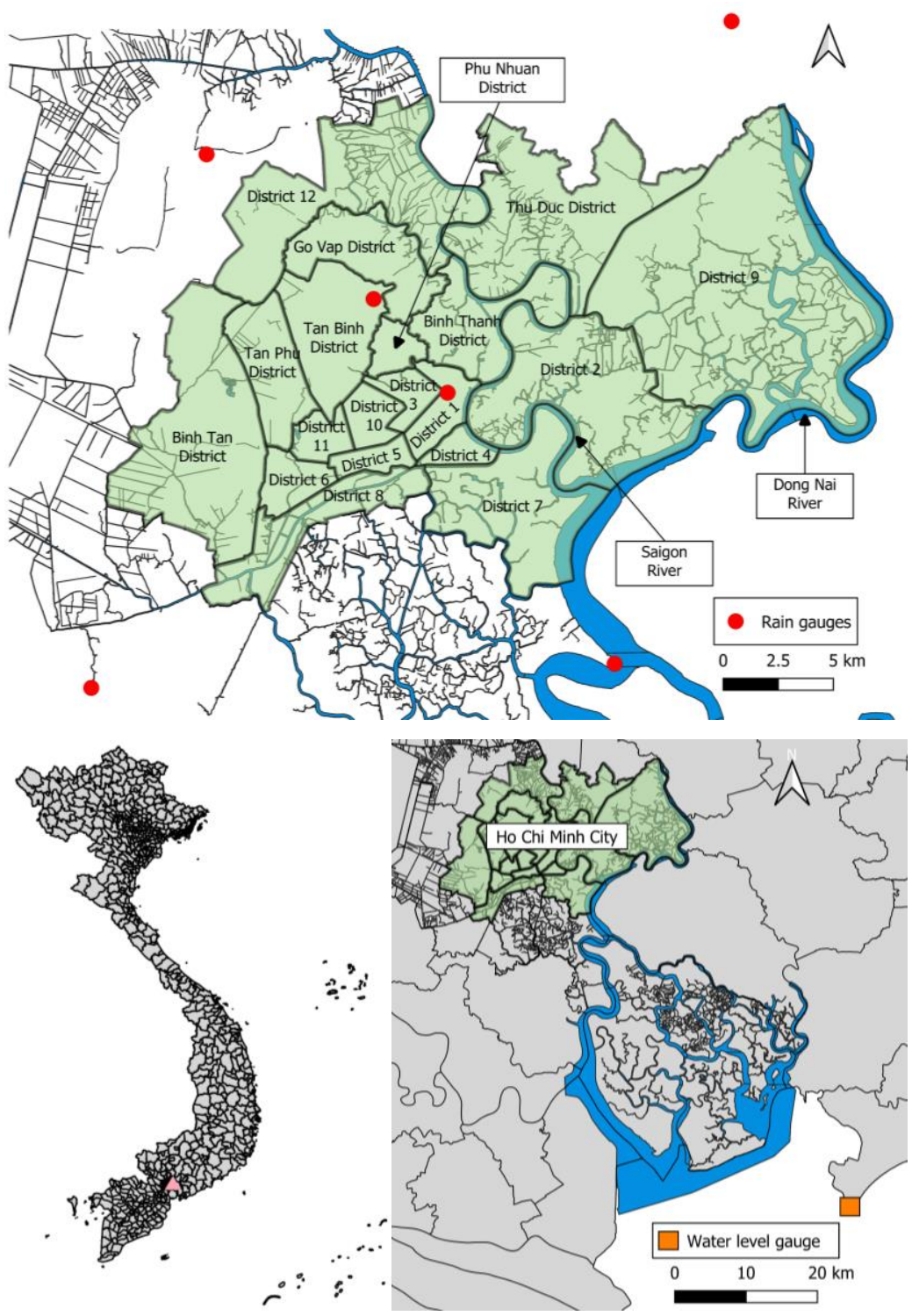

Figure 6.3 Study area (Ho Chi Minh City) where six rain gauges for collecting the daily rainfall over the city centre are denoted by red circles and a water level gauge by a yellow rectangle.

\subsubsection{Hydrodynamic modelling and simulation}

To estimate the flood inundation and risk driven by compound flooding under different scenarios, a well-calibrated TELEMAC-2D model (Hervouet, 2000) is employed, which has been already built based on our case (i.e., HCMC) and more details can be checked in Tran and 
Chau (2015). TELEMAC-2D model ${ }^{6}$ is a widely used 2D hydrodynamic model and can simulate the free-surface flows in two dimensions of horizontal space by solving the SaintVenant equations using the finite-element or finite-volume method with the triangular computation meshes (Briere et al., 2007; Teng et al., 2017; Vu et al., 2015).

The study domain of the TELEMAC-2D model includes the Saigon - Dong Nai river network, a digital elevation model (DEM) and a land cover map with different Chézy's roughness coefficients. The upper boundaries of the study domain are limited by the Tri An and Phuoc Hoa reservoirs on Dong Nai river, Dau Tieng reservoir on the Saigon River, Go Dau bridge on the Vam Co Dong river and Moc Hoa bridge on the Vam Co Tay River. The lower boundary extends to about $30 \mathrm{~km}$ away from the Soai Rap estuary to the East Sea. The computation of the model is in the VN2000 coordinate system (Thi et al., 2019) with 614,846 nodes and 1,223,200 triangle grids of different sizes according to the locations (the side length of grids is $50 / 100 \mathrm{~m}$ in the riverbed, 20/500 $\mathrm{m}$ in inland and 100/4000 $\mathrm{m}$ in East Sea). The initial water level is $+1.50 \mathrm{~m}$ and the calculation time step is $20 \mathrm{~s}$. Three types of inputs are required for flood simulation:

- Upstream discharges (m3/s): since the upstream discharge of HCMC can be controlled by the reservoirs, in this case, the values as the upper boundary of the model are constant and set following the local government recommendations.

- Downstream sea water level (m): it can be calculated by adding the designed skew surge obtained from the framework to the astronomical tide.

- Rainfall (mm): the designed rainfall obtained from the compound framework can be used directly.

\footnotetext{
${ }^{6}$ TELEMAC-2D model is built and the compound flood simulations in HCMC are run by T. Van Thu Tran*.

* Center of Water Management and Climate Change (WACC), VNUHCM-IER, Ho Chi Minh City, Vietnam.

* Email: ttvanthu@gmail.com
} 


\subsection{Results and discussion}

\subsubsection{Trend and correlation analysis}

Table 6.2 presents the results of the trend and correlation tests of all monthly maxima of the rainfall (MMR) and skew surge (MMS) in HCMC. Except for May, the monthly maxima of the skew surge time series were all detected to be increasing during the past 38 years at the significance level of 0.05 . According to Kendall's tau, there is not much difference in the magnitude of these positive trends between the dry and wet seasons on MMS. For MMR, the start and end months of the wet season, i.e., April, October, and November, show a positive trend. Regarding the correlation between MMS and MMR, March (the end month of the dry season) and April (the start month of the wet season) witness a significant, positive correlation, indicated by the $p$-values smaller than 0.05 from the Kendall or Spearman tests.

Table 6.2 Test and estimation results of monthly maximum rainfall (MMR) and skew surge (MMS) in HCMC.

\begin{tabular}{|c|c|c|c|c|c|c|c|c|c|c|c|}
\hline \multirow[t]{2}{*}{ Month } & \multirow{2}{*}{$\begin{array}{l}\text { Time } \\
\text { series }\end{array}$} & \multicolumn{2}{|c|}{ BBS-MK test } & \multicolumn{4}{|c|}{$\begin{array}{c}\text { Correlation test } \\
\text { (with all datasets) }\end{array}$} & \multicolumn{2}{|c|}{$\begin{array}{c}\text { Best-fitted } \\
\text { marginal } \\
\text { distribution }\end{array}$} & \multicolumn{2}{|c|}{ Best-fitted copula } \\
\hline & & $\begin{array}{c}\text { Kendall's } \\
\text { tau }\end{array}$ & $\begin{array}{c}p- \\
\text { value }\end{array}$ & $\tau$ & $\begin{array}{c}p- \\
\text { value }\end{array}$ & $\rho$ & $\begin{array}{c}p- \\
\text { value }\end{array}$ & Type & $\boldsymbol{\theta}$ & Copula & $\theta_{C}$ \\
\hline \multirow{2}{*}{ Jan } & MMS & 0.34 & 0.003 & \multirow{2}{*}{0.10} & \multirow{2}{*}{0.379} & \multirow{2}{*}{0.13} & \multirow{2}{*}{0.427} & GEV & $S$ & \multirow{2}{*}{ Independence } & \multirow{2}{*}{$\mathrm{S}$} \\
\hline & MMR & 0.11 & 0.326 & & & & & Gamma & $S$ & & \\
\hline \multirow{2}{*}{$\mathrm{Feb}$} & MMS & 0.45 & 0.000 & \multirow{2}{*}{0.09} & \multirow{2}{*}{0.445} & \multirow{2}{*}{0.14} & \multirow{2}{*}{0.403} & GEV & $S$ & \multirow{2}{*}{ Independence } & \multirow{2}{*}{$\mathrm{S}$} \\
\hline & MMR & 0.08 & 0.491 & & & & & Gamma & $S$ & & \\
\hline \multirow{2}{*}{ Mar } & MMS & 0.35 & 0.002 & \multirow{2}{*}{0.21} & \multirow{2}{*}{0.062} & \multirow{2}{*}{0.34} & \multirow{2}{*}{0.038} & GEV & $S$ & \multirow{2}{*}{ Gaussian } & \multirow{2}{*}{ NS } \\
\hline & MMR & 0.17 & 0.137 & & & & & Gamma & $S$ & & \\
\hline \multirow{2}{*}{ Apr } & MMS & 0.38 & 0.001 & \multirow{2}{*}{0.27} & \multirow{2}{*}{0.017} & \multirow{2}{*}{0.36} & \multirow{2}{*}{0.026} & GEV & NS1 & \multirow{2}{*}{ Clayton } & \multirow{2}{*}{ NS } \\
\hline & MMR & 0.47 & 0.000 & & & & & GEV & NS1 & & \\
\hline Mav & MMS & 0.16 & 0.167 & 002 & 0860 & 004 & 0823 & GEV & $S$ & Indenendence & $S$ \\
\hline May & MMR & -0.01 & 0.940 & 0.02 & 0.860 & & & GEV & $S$ & Independence & \\
\hline Jun & MMS & 0.41 & 0.001 & 008 & 0400 & 010 & 0550 & $\log N$ & $S$ & Plackett & $\mathrm{S}$ \\
\hline JuII & MMR & -0.08 & 0.497 & -0.00 & 0.490 & -0.10 & 0.039 & GEV & $S$ & r таскет & $\mathrm{S}$ \\
\hline Jul & MMS & 0.49 & 0.000 & -008 & 0.460 & $=10$ & 0562 & GEV & $S$ & & \\
\hline Juा & MMR & -0.06 & 0.615 & -0.08 & 0.469 & -0.10 & 0.562 & $\log N$ & $S$ & Independence & $\mathrm{S}$ \\
\hline Ang & MMS & 0.49 & 0.000 & 009 & 0453 & 014 & 0392 & GEV & $S$ & Rafte & $S$ \\
\hline Aug & MMR & -0.04 & 0.763 & & & & & $\log N$ & $S$ & Kaltery & \\
\hline
\end{tabular}




\begin{tabular}{|c|c|c|c|c|c|c|c|c|c|c|c|}
\hline \multirow{2}{*}{ Sep } & MMS & 0.44 & 0.000 & \multirow{2}{*}{0.07} & \multirow{2}{*}{0.532} & \multirow{2}{*}{0.10} & \multirow{2}{*}{0.568} & GEV & $\mathrm{S}$ & \multirow{2}{*}{ Raftery } & \multirow{2}{*}{$S$} \\
\hline & MMR & 0.20 & 0.083 & & & & & GEV & $S$ & & \\
\hline \multirow{2}{*}{ Oct } & MMS & 0.35 & 0.002 & \multirow{2}{*}{0.16} & \multirow{2}{*}{0.168} & \multirow{2}{*}{0.24} & \multirow{2}{*}{0.140} & GEV & $S$ & \multirow{2}{*}{ Joe } & \multirow{2}{*}{$S$} \\
\hline & MMR & 0.23 & 0.039 & & & & & GEV & NS1 & & \\
\hline \multirow{2}{*}{ Nov } & MMS & 0.25 & 0.031 & \multirow{2}{*}{0.13} & \multirow{2}{*}{0.257} & \multirow{2}{*}{0.18} & \multirow{2}{*}{0.276} & GEV & $S$ & \multirow{2}{*}{ Joe } & \multirow{2}{*}{$\mathrm{S}$} \\
\hline & MMR & 0.22 & 0.050 & & & & & GEV & $\mathrm{NS} 3$ & & \\
\hline \multirow{2}{*}{ Dec } & MMS & 0.38 & 0.001 & \multirow{2}{*}{0.21} & \multirow{2}{*}{0.059} & \multirow{2}{*}{0.29} & \multirow{2}{*}{0.075} & GEV & $S$ & \multirow{2}{*}{ Raftery } & \multirow{2}{*}{$\mathrm{S}$} \\
\hline & MMR & 0.22 & 0.053 & & & & & GEV & $S$ & & \\
\hline
\end{tabular}

Noted that GEV, Gamma, LogN are short for "Generalised extreme value distribution", "Gamma distribution" and "Lognormal distribution" respectively while S indicates stationary assumption and NS indicates the nonstationary assumption.

To reveal whether the correlation in March and April changes over time, I incorporated the Rolling Window method in the correlation test and the results with a window width of 30 years are shown in Figure 6.4. The correlation between MMS and MMR in March was firstly weakened before getting strengthened during the period around 1983-2012 to 1986-2015 and then became weak again in the final periods of 1987-2016 and 1988-2017. In April, the correlation in the first periods (1980-2009) were strong and became weak in the periods afterwards. 

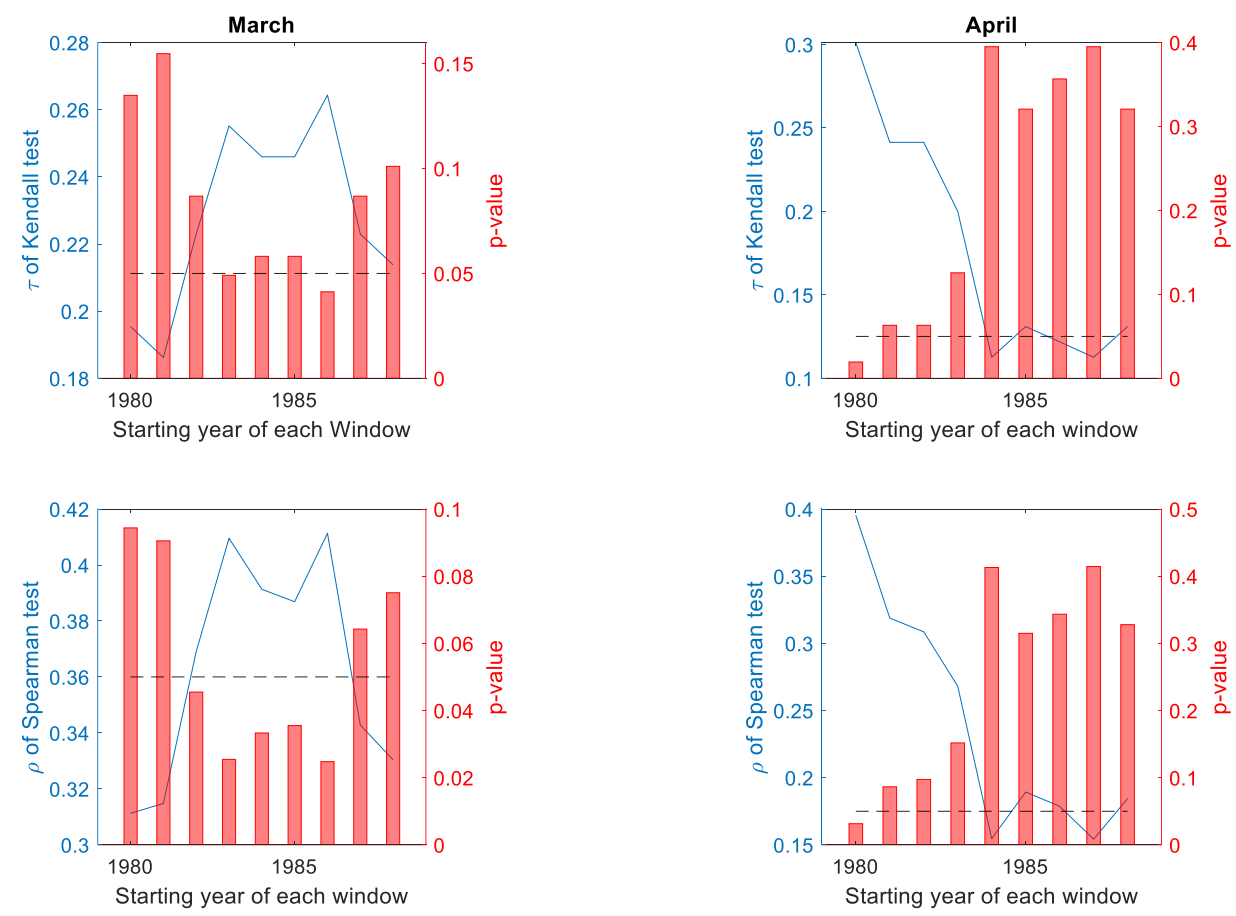

Figure 6.4 Trend analysis of correlation structure between MMS and MMR in March (left) and April (right) where the correlation coefficients $\tau$ of Kendall test and $\rho$ of Spearman test are depicted by blue curves and pvalues are indicated by red bars and the significance level $(0.05)$ is shown in dashed black line.

To analyse the impact from window width that could affect the correlation results, several different setups of the widths of moving window were used to test the consistency of the results which are presented in Figure 6.5. The $p$-value of both correlation tests using three different widths of the window in March and April, indicated by different colours. It can be observed that there is a small difference between the results tested by two methods (Kendall and Spearman). In March, the most correlation period is observed between 1987 to 2001 while in April, it is observed in the first several years, i.e., 1980 to 1990. It shows a general good consistency of testing results, but choosing a window width too short will lead to an inaccurate correlation test due to the limited number of data points while a width too long may cover up the possible correlative period. 

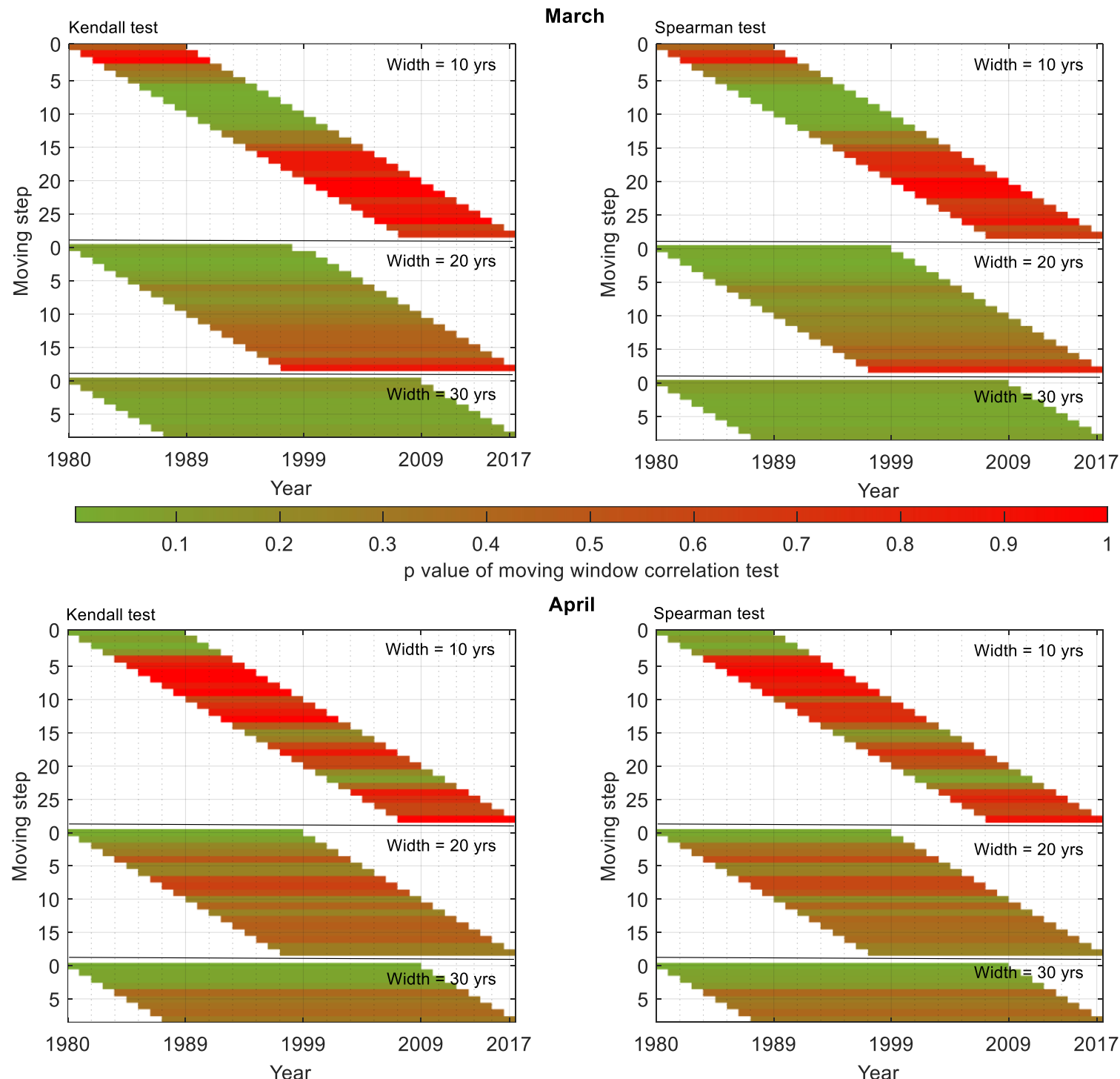

April

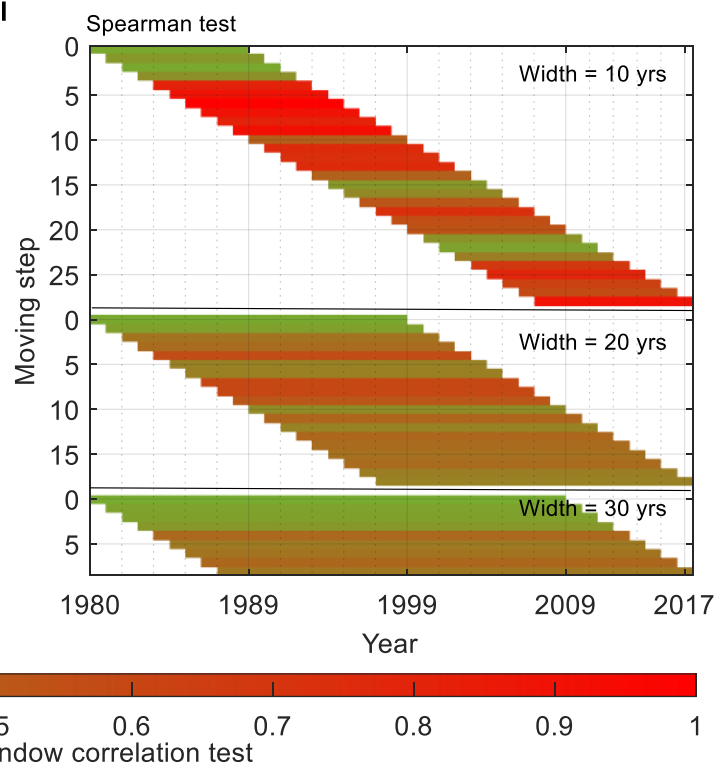

Figure $6.5 p$-value of two rolling window correlation tests (Kendall test and Spearman test) between monthly maxima of daily rainfall and skew surge in March and April.

In order to explain such a stronger and varying correlation between MMS and MMR in March and April, one possible reason is that both of them are directly affected by the easterly wind flow. This flow is perpendicularly toward the coastal area in the South of Vietnam only in these two months that strongly stresses the surface layer water into the mainland. This leads to a higher skew surge as presented in Figure 6.6. In addition, rainfall in these two months mainly 
comes from the perturbation and moisture from this easterly wind that facilitates convection. However, in other dry months, the wind is easterly to northeasterly and parallel to the coastal area while in wet months, this easterly component retreats to the middle to the northeast of Vietnam Eastsea while summer monsoon from Bengan Bay dominates the Vietnam area.

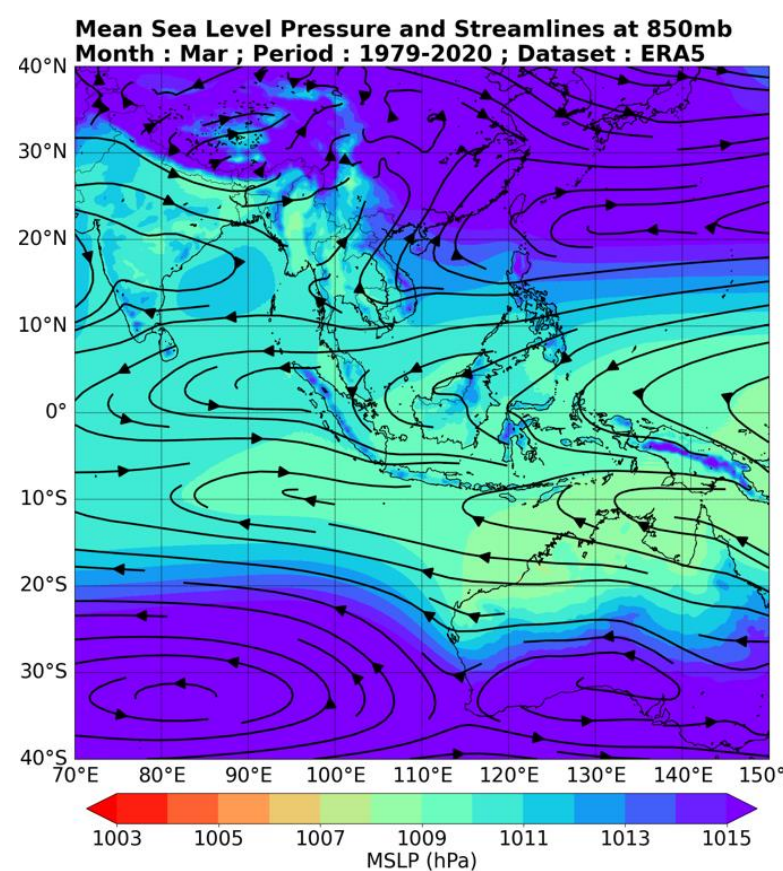

(a)

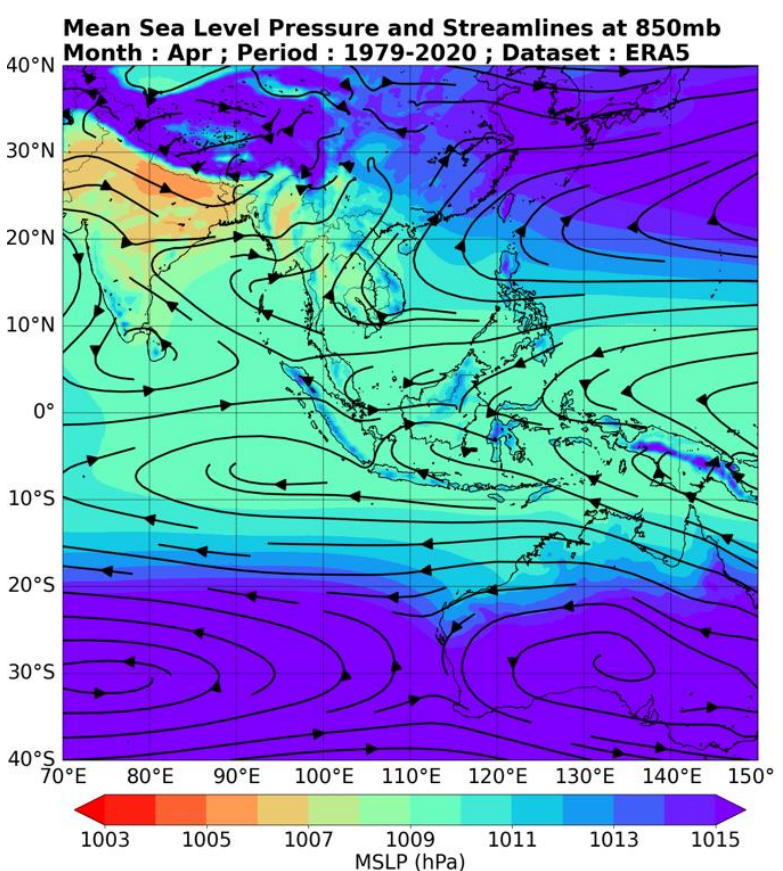

(b)

Figure 6.6 Mean sea level pressure and wind at $850 \mathrm{mb}$ for March (a) and April (b) averaged from 1979 to 2020 using the ERA5 dataset. $^{7}$

\subsubsection{Stationary and nonstationary joint probability distribution}

Boxplots of the original monthly maxima from both the skew surge and rainfall time series are depicted in Figure 6.7 where MMS shows much fewer variations compared with MMR which has a strong dry-wet season variation. As to the seasonal variation, the extreme cases in MMR (e.g., daily rainfall higher than $90 \mathrm{~mm}$ ) happen frequently in the wet season (e.g., August,

\footnotetext{
${ }^{7}$ The data were checked and the figure were generated by Linh N. Luu*.

* Royal Netherlands Meteorological Institute, De Bilt, The Netherlands;

Vietnam Institute of Meteorology Hydrology and Climate Change, Hanoi, Vietnam.

* Email: linhln.imhen@gmail.com
} 
September, October); in comparison, the extreme cases of skew surge occur mainly in the three dry months (February, March, April) and one wet month (July which has the largest deviation (around $0.4 \mathrm{~m}$ ) from the $5^{\text {th }}$ to $95^{\text {th }}$ percentiles).

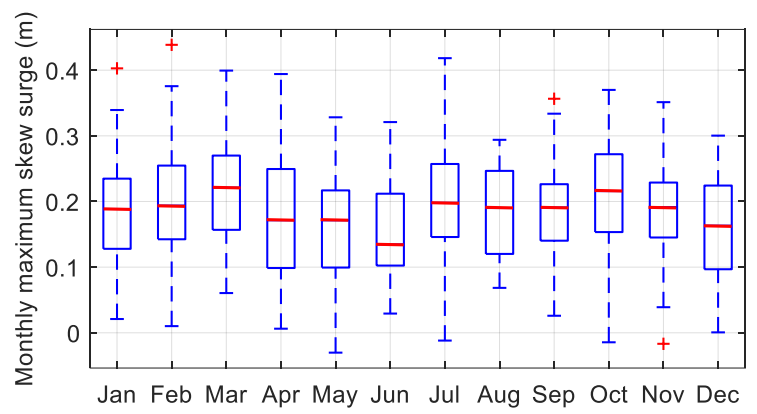

(a)

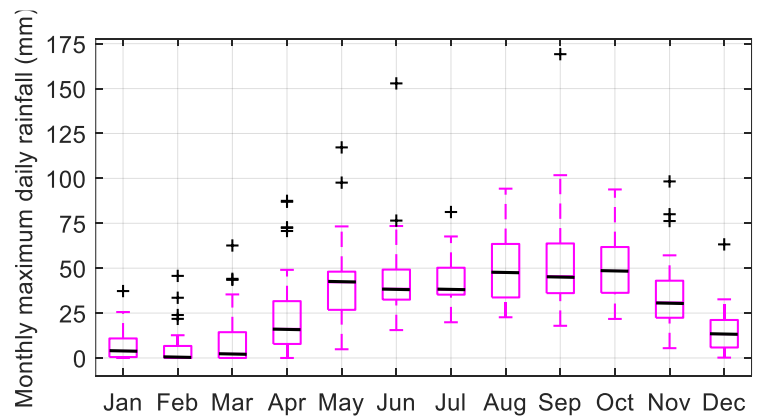

(b)

Figure 6.7 Distribution of monthly maximum time series of skew surge (a) and rainfall (b) (1980-2017). On each box, the central mark indicates the median, and the bottom and top edges of the box indicate the $25^{\text {th }}$ and $75^{\text {th }}$ percentiles, respectively, and the outliers are indicated by ' + '.

Following the trend analysis results discussed in the previous subsection, monthly maximum time series with an insignificant trend were fitted by a stationary model from all distribution candidates; those having a significant trend were fitted by both stationary and nonstationary models of all distributions. The best-fitted model was then selected using the AIC and BIC. The fitting results are presented in Table 6.2 and more details about parameter estimation and model selection are given in Appendix A.3. The results are further demonstrated in Figure 6.8 where four cases represented by four months are discussion follow below. 

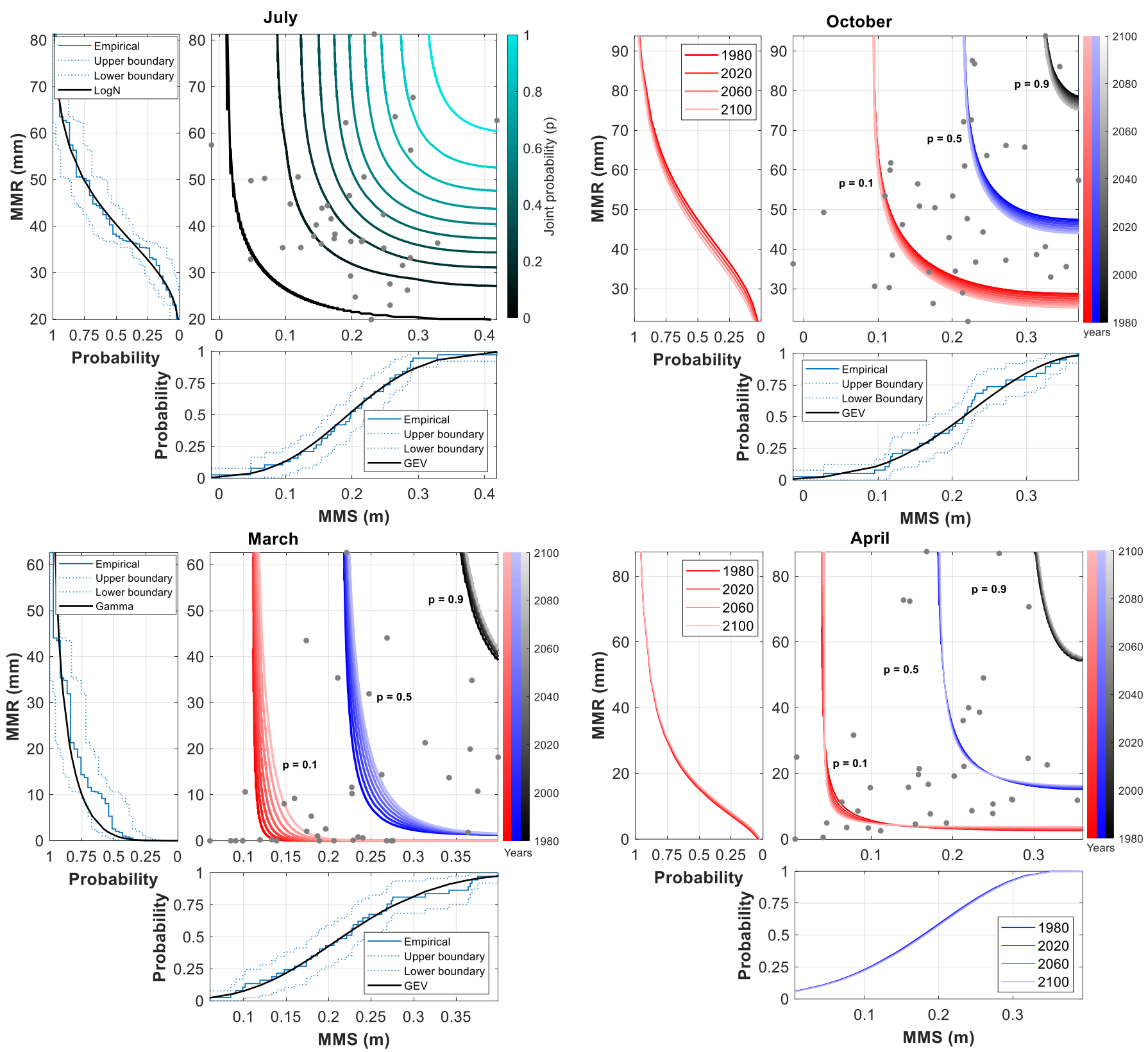

Figure 6.8 Quantiles corresponding to different joint cumulative probability ( $p=0.1,0.5$ and 0.9$)$ of the monthly maximum skew surge and rainfall under different assumptions in the selected four months where the best-fitted distributions of two marginals (MMS and MMR) are shown in the left and lower panels of each figure respectively: if the best-fitted distribution is stationary, the comparison between empirical and best-fitted distribution are shown while if it is nonstationary, only the best-fitted distribution is plotted and colour of curves indicates the changes over time (year).

\section{- Case 1: both marginals and the copula are stationary}


July is a wet month with the largest deviation of the skew surge. The best marginal distributions of MMS and MMR to characterise the features of July are stationary GEV and Lognormal distributions respectively. The correlation between MMS and MMR is insignificant and appear to be invariant with time therefore stationary independent copula is applied in this case. According to the AIC and BIC values, the correlation can be best described as independent and is depicted in Figure 6.8. The quantile curves corresponding to the joint exceedance probability are indicated by the colour from the dark ( $p=0.01)$ to the light $(p=0.99)$, which is generated by using the two marginal distributions whose empirical and best-fitted distribution are also presented.

\section{- Case 2: Nonstationary marginals but stationary copula}

October is near the end of the wet season with the right-skewed rainfall distribution and the left-skewed skew surge distribution. Both MMS and MMR are best fitted by GEV distribution and have a significant trend over time. However, only MMR supports the nonstationary assumption and the best model for it is GEV-NS1, i.e. the location parameter is assumed to be linearly changing over time. The correlation structure between MMR and MMS is insignificant and no significant correlation period is observed, therefore, the Joe copula is assumed to be stationary. The quantile curves of the joint probability are presented in Figure 6.8 where three joint exceedance probabilities are selected to view. The colour from the dark to the light indicates the time-varying of the joint probability from 1980 to 2017 and then extrapolating to the year 2100 for demonstration. Correspondingly, the marginal distribution of MMR is also time-varying. It can be observed that the time-varying marginal distribution can only cause an upward or downward movement of the quantile curves at each exceedance probability, but the shape of these curves is unchanged.

\section{- Case 3: Stationary marginals and nonstationary copula}

March is selected from the dry season with an extremely right-skewed rainfall and relatively symmetric-distributed skew surge. Unlike the other months, the correlation between MMR and MMS is significant but there are many zero values in the time series of rainfall therefore the correlation is tested by replacing these zeros with small random noises. The best-fitted marginal 
distribution is Gamma distribution for MMR and GEV distribution for MMS. As the correlation structure is significant and changes over time, a nonstationary Gaussian copula is assumed. The results show that the shape of quantile curves can vary and most of the changes occur in the middle than the tails. The combination of both higher rainfall and skew surge is increased in March with the time.

\section{- Case 4: Both marginals and the copula are nonstationary}

Although April belongs to the dry season, it has very limited zero value occurrence (only one zero value found) in the maximum rainfall series because this month is also the start of the wet season with a strong correlation between rainfall and skew surge. This correlation is found to be particularly strong over the first several years of the record then becomes weak. Therefore, a nonstationary correlation structure is assumed. For the marginal distribution, as the trends of MMR and MMS are both significant, I apply the nonstationary models and find only MMR prefers the nonstationary GEV-NS1 model with a decreased location parameter. The results show that the quantile curves twist over time where the change over time is translational at the lower tail of quantile curves (e.g., the combination of the same skew surge with higher rainfall) while at the middle, the angle of the curves shrinks (the combination of both lower skew surge and rainfall).

\subsubsection{Scenarios generation and compound flood simulation in HCMC}

To simulate the compound flooding response in HCMC under the nonstationary framework and mitigate the computational overheads, two months are selected, one from the dry and the other from the wet seasons (March and October) for this case study. The most likely scenario method is used for selecting the designed quantile pair of rainfall and skew surge with an exceedance probability corresponding to a return period of 50 years. Table 6.3 shows the scenarios of 2000, 2020, 2050 for March and October in HCMC for simulating compound floods, where $\mathrm{S}$ is short for skew surge and $\mathrm{R}$ is for rainfall. 
Table 6.3 The scenarios for compound flood simulation.

\begin{tabular}{|c|c|c|c|c|c|c|}
\hline \multirow{2}{*}{$\begin{array}{c}\text { Dry Season } \\
\text { (Mar) }\end{array}$} & \multicolumn{6}{|c|}{ Return level: 1 -in-50 years } \\
\hline & \multicolumn{2}{|c|}{ Time series } & Nonstationary & \multicolumn{2}{|c|}{ Time series } & Stationary \\
\hline \multirow{2}{*}{2000} & \multirow{2}{*}{ Case 1} & $\mathrm{~S}(\mathrm{~m})$ & 0.54 & \multirow{6}{*}{ Case 4} & \multirow{3}{*}{$\begin{array}{l}\mathrm{S} \\
(\mathrm{m})\end{array}$} & \multirow{3}{*}{0.52} \\
\hline & & $\mathrm{R}(\mathrm{mm})$ & 91.28 & & & \\
\hline \multirow{2}{*}{2020} & \multirow{2}{*}{ Case 2} & $\mathrm{~S}(\mathrm{~m})$ & 0.49 & & & \\
\hline & & $\mathrm{R}(\mathrm{mm})$ & 93.36 & & \multirow{3}{*}{$\begin{array}{c}\mathrm{R} \\
(\mathrm{mm})\end{array}$} & \multirow{3}{*}{90.87} \\
\hline \multirow{2}{*}{2050} & \multirow{2}{*}{ Case 3} & $\mathrm{~S}(\mathrm{~m})$ & 0.43 & & & \\
\hline & & $\mathrm{R}(\mathrm{mm})$ & 117.58 & & & \\
\hline $\begin{array}{c}\text { Wet Season } \\
\text { (Oct) }\end{array}$ & \multicolumn{2}{|c|}{ Time series } & Nonstationary & \multicolumn{2}{|c|}{ Time series } & Stationary \\
\hline \multirow{2}{*}{2000} & \multirow{2}{*}{ Case 5} & $\mathrm{~S}(\mathrm{~m})$ & 0.37 & \multirow{6}{*}{ Case 8} & \multirow{3}{*}{$\begin{array}{c}\mathrm{S} \\
(\mathrm{m})\end{array}$} & \multirow{3}{*}{0.37} \\
\hline & & $\mathrm{R}(\mathrm{mm})$ & 115.08 & & & \\
\hline \multirow{2}{*}{2020} & \multirow{2}{*}{ Case 6} & $\mathrm{~S}(\mathrm{~m})$ & 0.37 & & & \\
\hline & & $\mathrm{R}(\mathrm{mm})$ & 114.51 & & \multirow{3}{*}{$\begin{array}{c}\mathrm{R} \\
(\mathrm{mm})\end{array}$} & \multirow{3}{*}{106.81} \\
\hline \multirow{2}{*}{2050} & \multirow{2}{*}{ Case 7} & $\mathrm{~S}(\mathrm{~m})$ & 0.37 & & & \\
\hline & & $\mathrm{R}(\mathrm{mm})$ & 113.66 & & & \\
\hline
\end{tabular}

After generating the designed pair of rainfall and skew surge, the next step is to transform this designed pair to appropriate boundary conditions for the model. Since the skew surge cannot be used directly as the input of the TELEMAC-2D model, I broadly followed the method presented in Fox (2009) and analysed all astronomical tide and the 24-hours sea water level profiles in March and October over the 38 years. The astronomical tides are generated by using the MATLAB UTide (Unified Tidal Analysis and Prediction Functions) package (Codiga, 2011) when processing the skew surge data (more details can be checked in Couasnon et al. (2021)). The tide profile of the dates and the tidal period when the monthly maximum skew surge happened is explored to find the most frequent tide profile in these two months from 1980 to 2017. In addition, the exact time when the maximum skew surge happened is also recorded.

Results show that there are two typical profiles in March as shown in Figure 6.9 and three typical profiles in October (see Figure 6.10). It can be observed that for 24 hours, Type 1 of Figure 6.9 has two positive peaks with an average tide range of $2.5 \mathrm{~m}$ while Type 2 has a flat positive peak with an average tide range of $2 \mathrm{~m}$. And for October, the highest average tide level 
is in Type 3 with two positive peaks. Following the advice from Tran (ttvanthu@gmail.com) who contributes to the model simulation, the practical tide in $\mathrm{HCMC}$ is the profile with two highs and two lows. Therefore Type 1 of March and Type 3 of October are selected as the basic profiles.

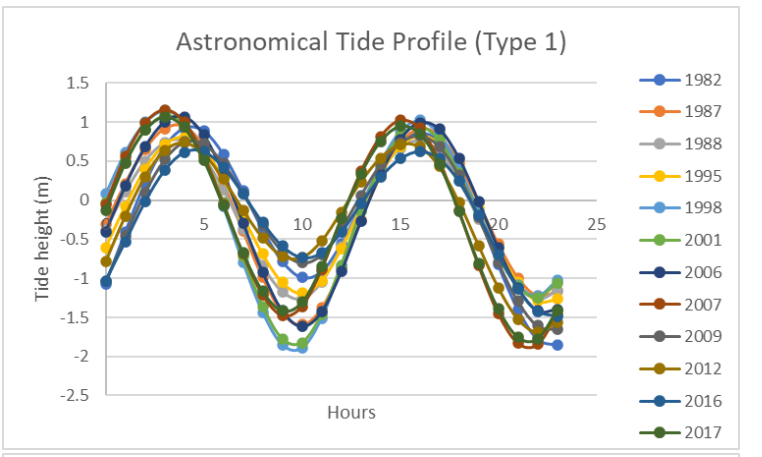

Astronomical Tide Profile (Type 1, average)

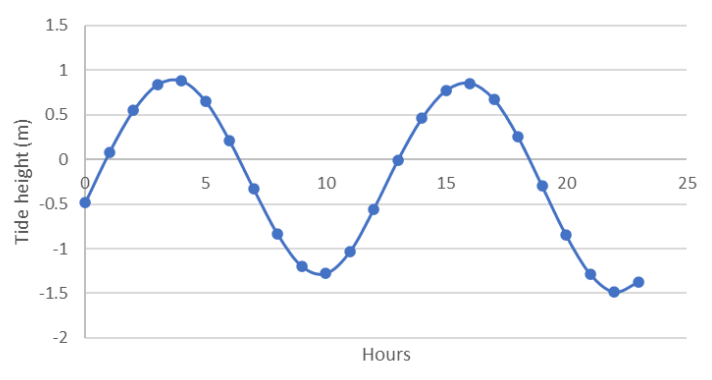

(a)

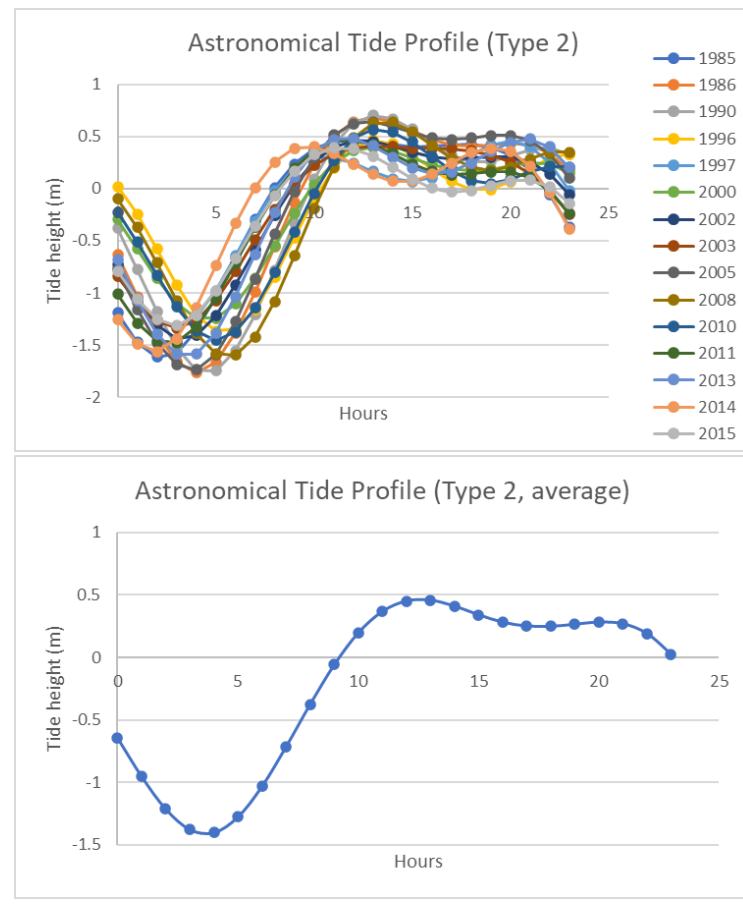

(b)

Figure 6.9 Two typical astronomical tide profiles in March.
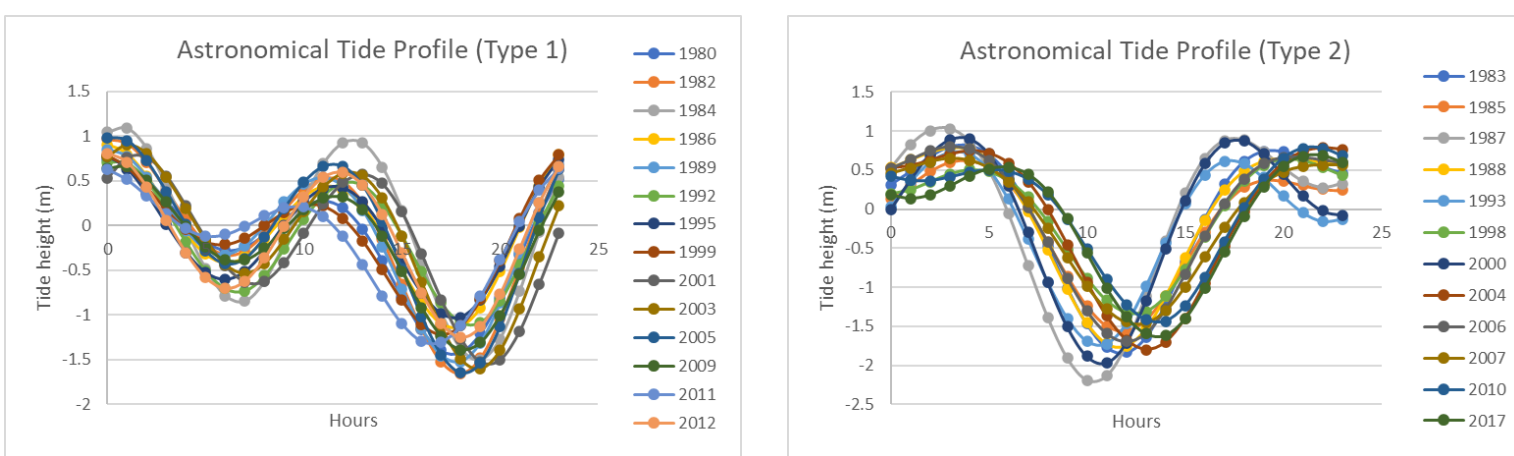


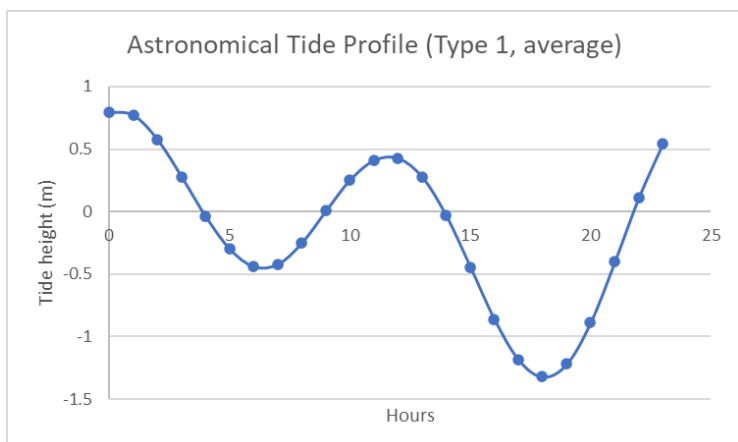

(a)

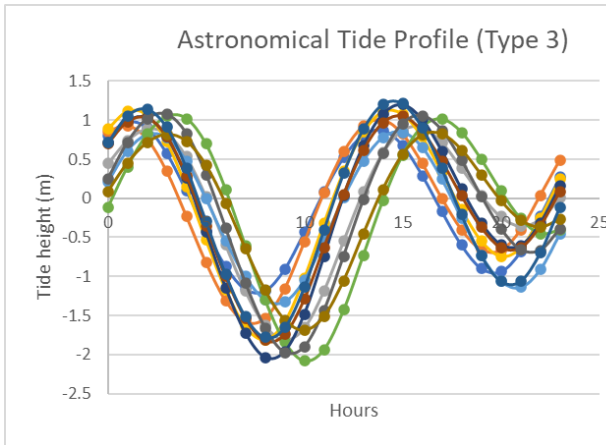

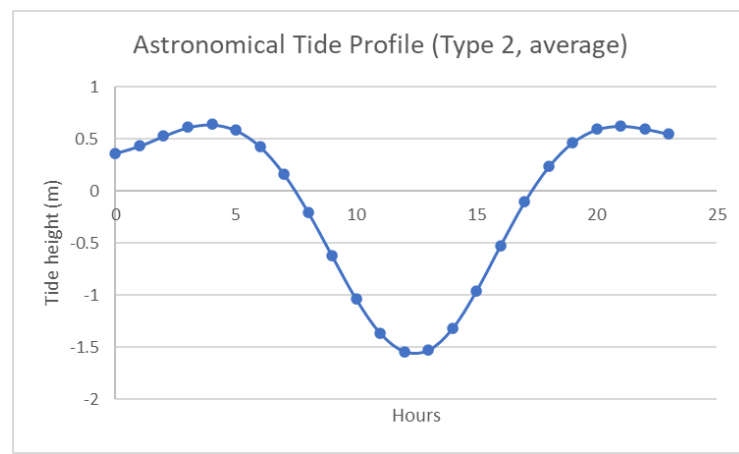

(b)

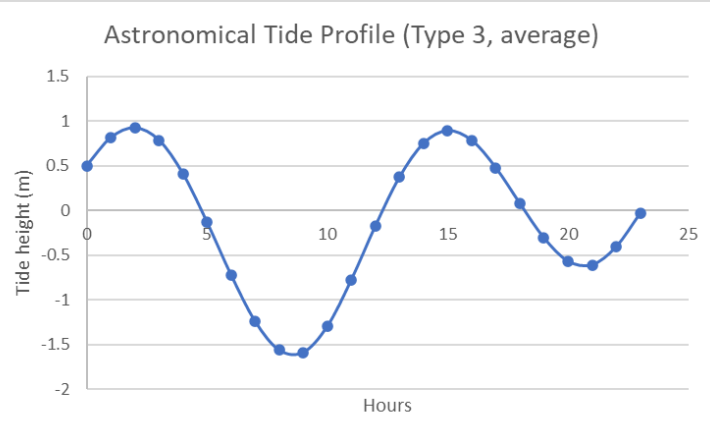

(c)

Figure 6.10 Three typical astronomical tide profiles in October.

Meanwhile, it is also found that the maximum skew surge in most situations occur near the second peak of the tide (around 63\% happened at the second peak and in $80 \%$ of cases, there is no time difference between the water level peak and tide peak). To consider the worst situation where the potential flood damage is high, the skew surge estimated by the nonstationary multivariate framework is added to the top of the second peak of the typical tide (Table 6.3) and interpolated to the other time points (24 hours). For all different scenarios, sea level rise (SLR) is also added to get the final sea water level for the input of the TELEMAC2D model to construct the three nonstationary scenarios for each month. And the values of SLR are selected from the climate report released by the Ministry of Natural Resources and Environment, Vietnam, i.e., 0.12 for the year 2020 and 0.33 for 2050 relative to the period of 1980-1999 of high $\mathrm{CO}_{2}$ emission (MNRE, 2009).

To compare with what would have been produced with the conventional approach, i.e., without taking into account the non-stationarity, I also generated a stationary case for the two months, i.e., re-fitting the marginal distributions and copula whose parameters are all constant. 
To explore the possible worst situation of compound flooding driven by a design rainfall and skew surge event, the TELEMAC-2D model is run multiple times with different time lags (e.g., $15 \mathrm{~min}, 30 \mathrm{~min}, 1 \mathrm{hr}, 2 \mathrm{hrs}$ ) between the peak of the rainfall events and water level peaks measured at Phu An station near the city centre. The results show that the worst situation usually occurs when the rainfall event is ahead of the surge by $2 \mathrm{hr} 15 \mathrm{~min}$.

The simulation results of the 1-in-50-year compound flood estimated by the proposed nonstationary framework compared with the stationary case in March and October are presented in Figure 6.11.

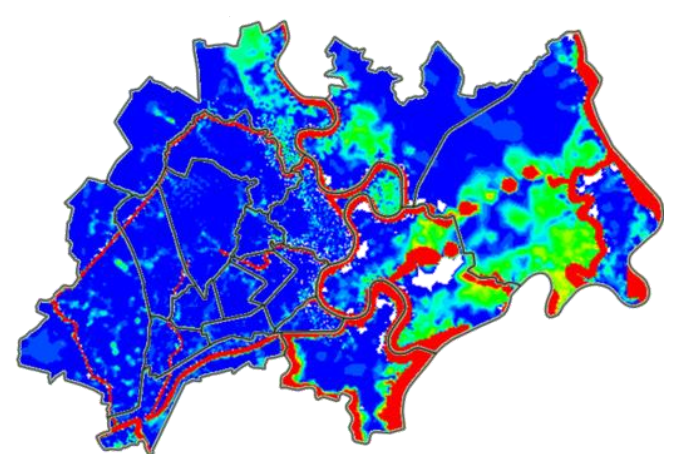

(a) Stationary (March)

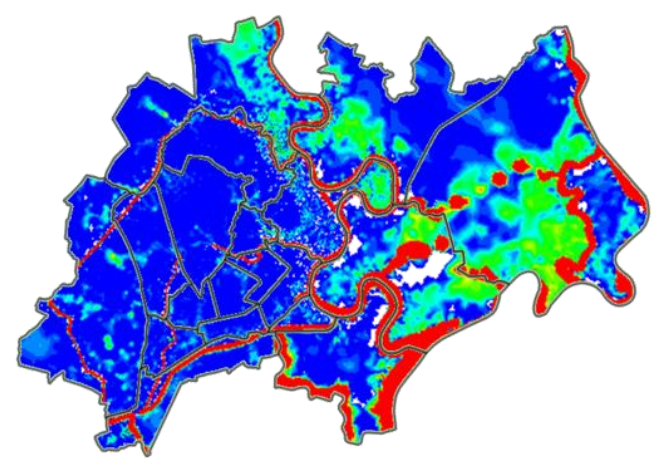

(c) Stationary (October)

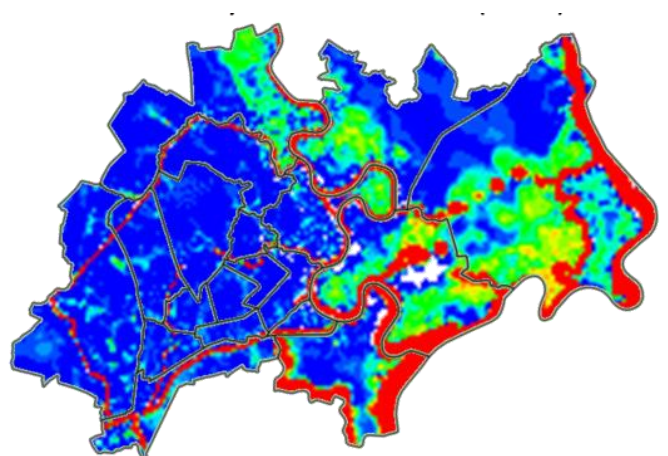

(b) Nonstationary for 2050 (March)

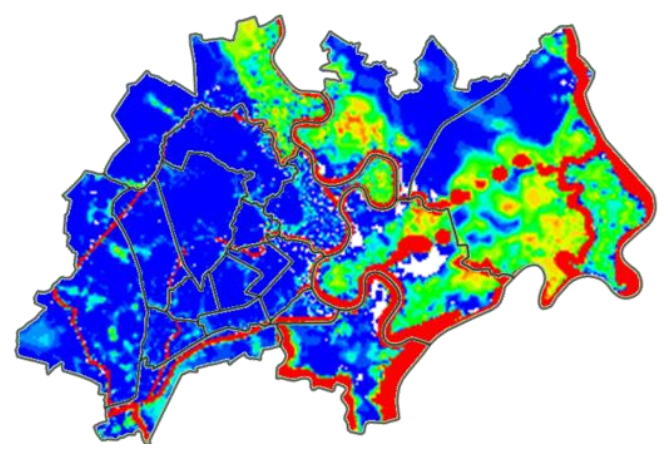

(d) Nonstationary for 2050 (October)

Flood Depth

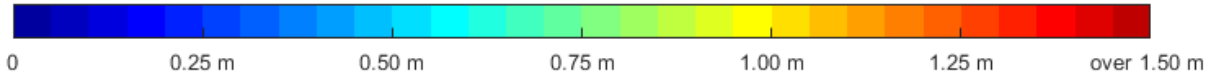

Figure 6.11 Flood inundation maps compared between the stationary and nonstationary cases in March (a and b) and October (c and d). 
It can be observed that the eastern regions of HCMC are more affected by the floods, especially in the confluent area of Saigon and Do Nai Rivers which are marked as red colour. Regards to the inundation areas, floods simulated in October are more significant than in March, which is consistent with the common sense that the wet season receives more water than the dry seasons. However, by comparing the flood inundation maps simulated by the stationary case and nonstationary case for the year 2050, remarkable underestimates from the stationary case can be observed in both months, particular in October. Such underestimation can be attributed to different reasons: for March, there is a time-varying correlation between the maximum rainfall and skew surge (see Figure 6.8, although the distributions of rainfall remain unchanged), which results in increased compound flood risks in the perspective of non-stationarity; In October, the correlation between the rainfall and skew surge is not as significant nor does it change over time but the rainfall itself is nonstationary, which leads to a faster increase in flood depth than March over time.

In general, the stationary case has a slight underestimation in the current scenario (2020) and a remarkable underestimate in the future scenario (2050). These findings underpin the importance of incorporating the non-stationarity into the compound flood estimation because not only the driver itself may change alongside the climate change, but their correlation can also vary due to many implicit factors which need further exploration.

\subsection{Summary and remarks}

This chapter presents the development of a nonstationary multivariate modelling framework for quantifying the time-varying joint probability of two meteorological and oceanographic drivers of compound flooding in view of their non-stationarity. The framework utilises the Bootstrapping Mann-Kendall trend test and the rolling window correlation tests to determine whether the marginals and/or their dependence structure should be treated in a stationary or non-stationary fashion. The Bayesian Markov-Chain Monte-Carlo (B-MCMC) method is applied to estimate the nonstationary parameters and the associated uncertainty. Best marginal distribution and copula are selected by evaluating Akaike's information criterion (AIC) and 
Bayesian information criterion (BIC). To present the applicability of this framework, I applied it to the case of Ho Chi Minh City (HCMC), Vietnam to estimate the compound flooding caused by two drivers, i.e., monthly maxima of inland precipitation and skew surge in both dry and wet seasons. The flood depths are simulated by a well-calibrated hydrodynamic TELEMAC-2D model using two designed driver values at a return level of 50 years. I compare the scenarios generated by the nonstationary case with the stationary case produced by the framework, as well as the simulation results and find that:

1) The correlation between the monthly maximum rainfall and skew surge is independent except in March and April which are the boundary between the dry season and wet season. Such the correlation in March becomes more significant in the last 10 years while in April it becomes less significant.

2) The series of monthly maximum rainfall in October and November have been changing over time and their distribution can be best fitted by nonstationary generalised extreme value (GEV) model of which only the location parameter (linking to the most frequent level of monthly maximum daily rainfall) is time varying.

3) The simulation results of the HCMC case study show that the traditional stationary approach produces remarkable underestimates in simulating the compound flood depth in the current (2020) and future (2050) scenarios. The dry month of March is expected to get more floods similar to the wet month of October, which may lead to that the wet season in $\mathrm{HCMC}$ is expected to shift earlier.

The developed nonstationary framework offers a great deal of flexibility for modelling complicated hydroclimatic extreme variables as far as the possible combinations of stationary and nonstationary assumptions are concerned. Compared with other approaches, the B-MCMC method that is employed in the framework can be more effective to process the complex timevarying phenomena under climate change such as estimating the correlation structure between continuous and discrete variables. This paper presents the first application to evaluate compound flooding driven by extreme rainfall and skew surge in the perspective of nonstationarity in HCMC. Other low-lying coastal cities and countries may also confront a similar predicament where a comprehensive regional risk assessment of the compound flooding 
potential is currently missing. This modelling framework, with the flexibility it has, will be of substantial use in this regard. The study also underpins that climate change can affect not only the hydrometeorological or oceanographic extremes themselves but also their interaction which tends to become either more correlated or independent. As far as managing compound flood risk is concerned, relevant authorities should carefully consider such consequences arising from climate change and evaluate their current strategies that may have been historically produced from a stationary perspective.

As with any statistical analysis application, data length and quality can inevitably affect the results, so can the uncertainty in physical modelling. It should be recognised that a relatively short length (38 years) of data is used in this study. Although this does not affect the concept of the modelling framework I develop, direct use of the results from this study for policymaking purposes should consider the caveat above into the context. As such, further work is recommended to investigate compound flood and correlation between rainfall and surge with longer-term observations which are likely to make the conclusions more robust. And for the multivariate nonstationary framework, more copulas and types of distribution candidates can be involved alongside uncertainty quantifications. Apparently, linking climate model projections into the framework will be another important and challenging area to explore. 


\section{Part II}

Pattern recognition on hydroclimatic extremes 



\section{Chapter 7 Identification and Classification of}

\section{Hydroclimatic Extremes Using Pattern Recognition}

\section{and Convolution Neural Networks}

According to the quantitative results presented in Chapter 3 and Chapter 4, the spatial and temporal rainfall distribution can be bounded within several specific patterns. Therefore, identifying and classifying such patterns is of great value to help understand the underlying mechanism and improve the forecasting capability. Motivated by this, in this chapter, an effective pattern recognition technique by incorporating an unsupervised clustering algorithm, i.e., Spatial Pattern Extraction and Recognition (SPER) toolbox, is developed to process automatic identification and classification of extreme patterns by extracting their spatial and physical features and answer the research question Q6. This chapter also demonstrates three case studies to show the efficiency and application of the toolbox. Further, the SPER toolbox presents great potential in auto-labelling clusters to support deep/machine learning of complex environmental spatial-temporal features over large datasets, demonstrated by an example of training a convolution neural network $(\mathrm{CNN})$ which can recognise new rainfall patterns and classify them to the learned catalogues with high accuracy.

\subsection{Introduction}

Rainfall is one of the most essential variables in climate and water resources research, which has a complex and nonlinear relationship with other various meteorological and climatological 
variables and topographic conditions (Kirono et al., 2010). Many approaches and models have been developed for simulating and predicting rainfall based on such relationships. For example, the generalised linear model (GLM) is a widely-used tool for quantifying the relation between rainfall and other hydroclimatic predictors (Urdiales et al., 2018; Yan et al., 2002; Yunus et al., 2017) such as temperature, potential evaporation, wind speed, El Niño, La Niña, and Southern Oscillation indices; as well as spatial predictors (Beecham et al., 2014; Kenabatho et al., 2017) such as altitude, geographic location, size and shape shown in Chapter 3. However, as the topography does not frequently change over time and the large-scale structure of atmospheric circulation remains relatively stable, the spatial and temporal rainfall distribution can be bounded within several specific patterns. For example, the rainfall patterns in the windward regions are normally different from those in the leeward region, especially in the mountainous area (Lin et al., 2017); desert areas usually receive limited rainfall because of high temperature heating the descending air which hinders the formation of clouds that cause rainfall. Therefore, identification and classification of such rainfall patterns are of great value to help understand the underlying mechanism and improve the forecasting capability, especially for those based on deep learning methods which have seen a fast-growing number of applications recently. For example, in hydroclimatic science, some researchers applied machine learning methods such as artificial neural networks (ANN) to extract features of spatiotemporal climatic variables (Qiu et al., 2017); others parameterized those features (Gentine et al., 2018; O'Gorman and Dwyer, 2018) to realize weather predictions (Liu et al., 2016; Petersik and Dijkstra, 2020).

Appropriate training is essential and often challenging in the application of these deep learning applications, in an effort of achieving accurate and reliable prediction, and expanding the applications in climate science. Unlike the data traditionally used to develop neural network algorithms such as face images for facial recognition and classification, which have commonalities and specific features that can be easily classified, the hydro-climatic data from model projections and field observations are often highlighted by their intermittent spatial variations, and often chaotic, nonstationary, multi-scale temporal distributions.

The other main challenge is due to the amount of training data required by deep learning techniques. For example, a supervised convolution neural network $(\mathrm{CNN})$ usually demands a 
large dataset for training, which should be all pre-catalogued into different labels/classes. This apparently requires huge time and labour if done manually. To address these issues and research question Q6, I designed a toolbox named "Spatial Pattern Extraction and Recognition (SPER)" by incorporating an unsupervised clustering technique, the K-means cluster method into a spatial feature identification algorithm. The SPER toolbox allows the large-scaled input of grid-based hydroclimatic dataset (observed or climate projected) and employs the image thresholding segmentation method to help reduce the overhead of sampling data for training. The significant pattern whose hydro-climatic variable is over the defined threshold is extracted and regarded as the region of interest (ROI) within a geographic boundary which can be automatically detected. For each ROI, both spatial features (i.e., the geographic location, size, orientation, shape of ROI) and hydrologic features (i.e., total volume, areal average value and spatial distribution of the hydrological variable in the ROI) are identified and calculated and the final classification of the extreme patterns is based upon these features. Three example cases are included in this chapter to present the applicability of the SPER toolbox, i.e., 1) a catchment-based analysis of extreme rainfall in England and Wales where more than 900 catchments were categorized by the spatial features extracted from the SPER toolbox and the variation of extreme rainfall with respect to catchment location, size, orientation and shape was quantified; 2) pattern recognition of daily rainfall over the last century in Great Britain where the top 3 dominating patterns were recognized; 3) Tracing rainfall area and spatial distribution in 24 hours in Guangdong, China where the track of the rainfall centre and how the rainfall area changed were presented with the support of the SPER toolbox. Besides, the potential of the toolbox for supporting machine learning is discussed with an example given to demonstrate how the toolbox can be employed to pre-catalogue the hydroclimatic patterns into different labels which are used for training a supervised artificial neural network. The examples given in this chapter can be readily expanded to cover many other similar use cases.

The remainder of this chapter is organised as follows: Section 7.2 describes the design of the Spatial Pattern Extraction and Recognition (SPER) toolbox; Section 7.3 illustrates three cases for demonstrating the application of the toolbox while its potential to support machine learning 
is discussed in Section 7.4. Finally, the conclusions and access to the toolbox are given in Section 7.5.

\subsection{The design of the SPER toolbox}

The Graphical User Interface (GUI) of Spatial Pattern Extraction and Recognition (SPER) toolbox as well as the main functions are presented in Figure 7.1. Users can input the gridbased hydroclimatic dataset and its resolution (e.g., the grid size of the dataset), and predefine a threshold value for extraction. The next step is to extract the hydroclimatic variables over the threshold located within a closed boundary which forms a region of interest (ROI). Then the SPER toolbox can automatically detect the location of each such ROI and quantify the spatial features including the geographic location, size, orientation, and shape of ROI and the hydroclimatic features such as areal average value, total volume, and spatial distribution of the hydroclimatic variable. The toolbox can also demonstrate the ROI selected by users. An example plot is presented in the right panel in GUI shown in Figure 7.1. All the information of ROIs extracted from the input datasets can be saved in a text file if needed.

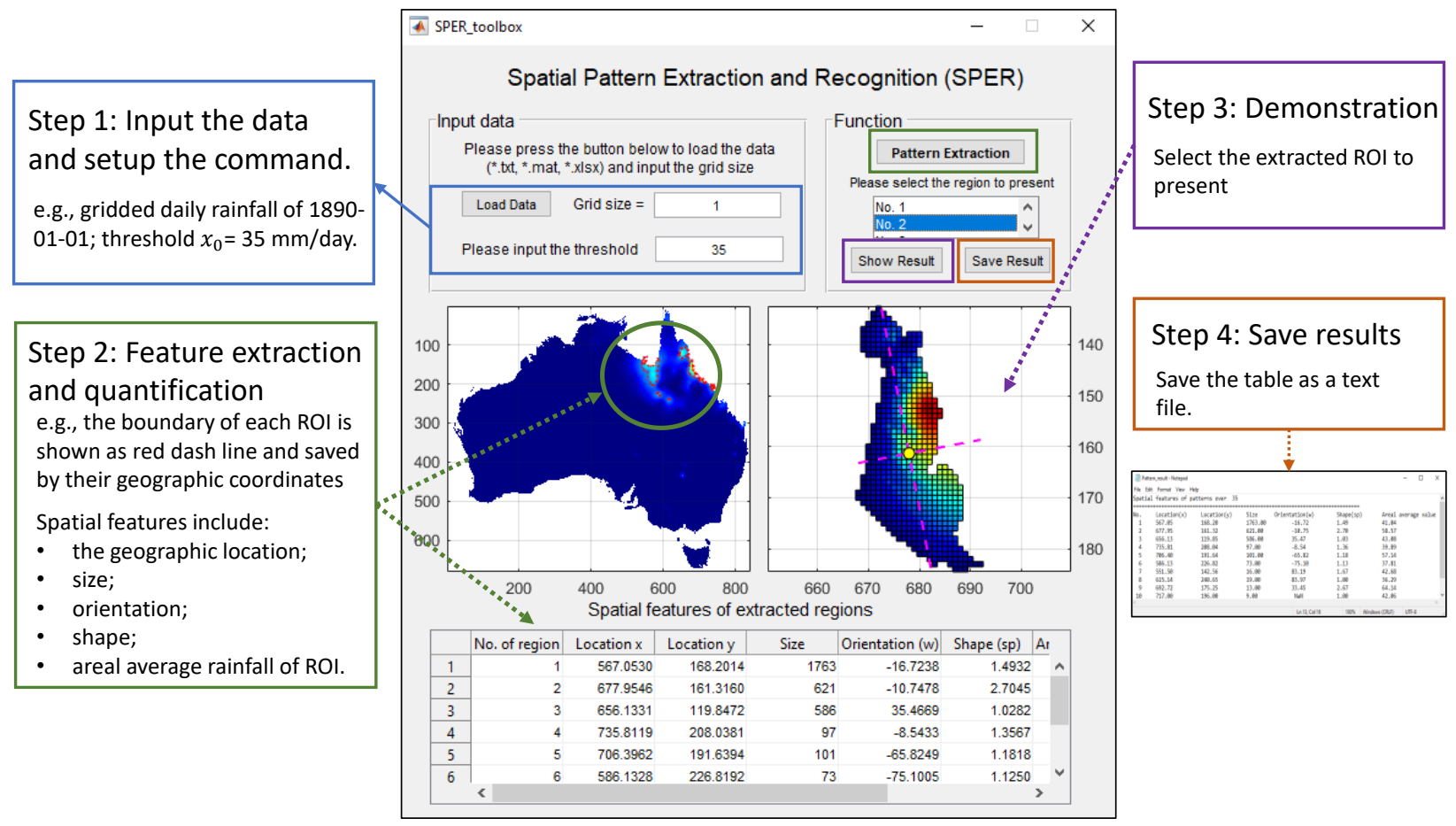


Figure 7.1 SPER: Spatial Pattern Extraction and Recognition Toolbox.

\subsubsection{Thresholding segmentation and boundary detection}

There are two different types of inputs to the toolbox, i.e., as datasets or as images. If the input is grid-based dataset, after predefining the threshold value $x_{0}$, the first step of segmentation is to subdivide the dataset into continuous regions, so-called regions of interest (ROIs). One of the most widely used method of extracting an object from the background is to distinguish different modes according to the threshold $x_{0}$ (Panu et al., 1978; Weszka and Rosenfeld, 1978). Motivated by this, the toolbox divides all grids in the dataset into two types: any grid $(x, y)$ for which $f(x, y) \geq x_{0}$ is called an object grid otherwise is a background grid. Thus, the new dataset $g$ can be defined as:

$$
g(x, y)=\left\{\begin{array}{l}
f(x, y), \quad \text { if } f(x, y) \geq x_{0} \\
\mathrm{NaN}, \quad \text { if } f(x, y)<x_{0}
\end{array}\right.
$$

where NaN means no data, which indicates the background.

Then the segmentation is implemented based on the discontinuity and similarity of the grid values of the dataset. The discontinuity is usually defined as an abrupt change of grid values nearby, which can be used for detecting the boundary of the pattern while the similarity is where the grids show similar properties (e.g., object or background). An exemplification sketch is presented in Figure 7.2a where to the new dataset $g$, an isolated ROI where $f(x, y) \geq 35$ (blue grids) is defined as an area with the similar property and its boundary is detected by an abrupt discontinuity (highlighted as red grids in the sketch a) where no data are founded. 


\begin{tabular}{|l|c|c|c|c|c|c|}
\hline $\mathrm{NaN}$ & $\mathrm{NaN}$ & $\mathrm{NaN}$ & $\mathrm{NaN}$ & $\mathrm{NaN}$ & $\mathrm{NaN}$ & $\mathrm{NaN}$ \\
\hline $\mathrm{NaN}$ & $\mathrm{NaN}$ & 35 & 35 & $\mathrm{NaN}$ & $\mathrm{NaN}$ & $\mathrm{NaN}$ \\
\hline $\mathrm{NaN}$ & 35.3 & 36.6 & 36.8 & 35.8 & $\mathrm{NaN}$ & $\mathrm{NaN}$ \\
\hline $\mathrm{NaN}$ & 36.2 & 38.1 & 38.1 & 36.5 & 35.1 & $\mathrm{NaN}$ \\
\hline $\mathrm{NaN}$ & 36.1 & 36.3 & 37.7 & 36.3 & $\mathrm{NaN}$ & $\mathrm{NaN}$ \\
\hline $\mathrm{NaN}$ & 35.3 & $\mathrm{NaN}$ & 36.2 & 35.4 & $\mathrm{NaN}$ & $\mathrm{NaN}$ \\
\hline $\mathrm{NaN}$ & $\mathrm{NaN}$ & $\mathrm{NaN}$ & $\mathrm{NaN}$ & $\mathrm{NaN}$ & $\mathrm{NaN}$ & $\mathrm{NaN}$ \\
\hline
\end{tabular}

(a)

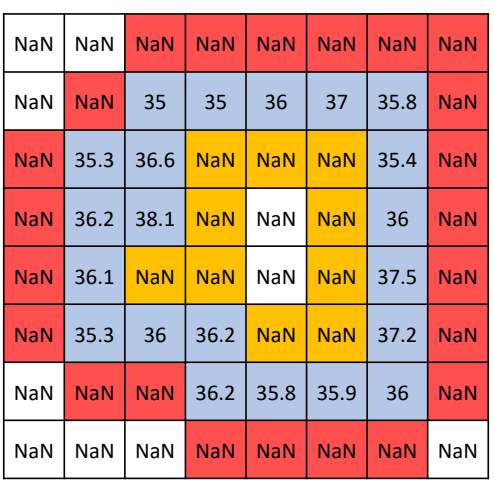

(b)

Figure 7.2 Two example sketches of ROI where $f(x, y) \geq 35$ without (a) and with (b) hole.

If some background grids $(f(x, y)<35)$ within the ROI boundary, the area constituting by these grids is called a "hole". Such a hole can also be detected straightforwardly due to the discontinuity at the intensity level of the dataset. As the example mask shown in Figure 7.2b, the yellow grids are the hole boundary while the blue grids are the ROI which is an inner connected region greater than the defined threshold. The information of each boundary (e.g., the coordinates of the boundary grid) is recorded separately and stored.

\subsubsection{The algorithm for extracting and quantifying the spatial features of ROI}

Each ROI extracted from the last step can be simplified as an irregular polygon and the algorithm for quantifying the spatial features is described in the four steps below:

- Step 1: Decompose the irregular ROI inside the boundary $\left(x_{j}, y_{j}\right)$ into many (e.g., $\left.i\right)$ small regular polygons $A_{i}$ such as rectangle and triangle then the geometric centroid $\left(C_{x}, C_{y}\right)$ of ROI can be calculated as:

$$
\begin{aligned}
& C_{x}=\sum C_{i x} A_{i} / \sum A_{i}=I_{x} / \sum A_{i}=\frac{1}{6} \sum_{j=1}^{n}\left(x_{j} y_{j+1}-x_{j+1} y_{j}\right)\left(y_{j}+y_{j+1}\right) / \sum A_{i} \\
& C_{y}=\sum C_{i y} A_{i} / \sum A_{i}=I_{y} / \sum A_{i}=\frac{1}{6} \sum_{j=1}^{n}\left(x_{j} y_{j+1}-x_{j+1} y_{j}\right)\left(x_{j}+x_{j+1}\right) / \sum A_{i}
\end{aligned}
$$


where $n$ is the number of vertices of the irregular ROI, i.e., the number of boundary points in $\left(x_{j}, y_{j}\right) ; C_{i}$ indicates the centroid of each divided polygon which can be found easily and the subscripts $x$ or $y$ mean the distance of the centroid from the $y$-axis (the vertical axis) or the $x$ axis (the horizontal axis); $C_{i x} A_{i}$ and $C_{i y} A_{i}$ are also known as the first moment of the area with respect to $y$ and $x ; \sum A_{i}$ is the total area of ROI which is a sum of the area of divided polygons.

- Step 2: Calculate the second moment of area of ROI which is used for defining the principal axes of ROI and the rotation thereby obtaining the orientation of ROI. The second moment of the area with respect to the centroid $\left(C_{x}, C_{y}\right)$ is given by (Hally, 1987):

$$
\begin{gathered}
I_{x x}=\frac{1}{12} \sum_{j=1}^{n}\left(x_{j} y_{j+1}-x_{j+1} y_{j}\right)\left(y_{j}{ }^{2}+y_{j} y_{j+1}+y_{j+1}{ }^{2}\right)-I_{y}{ }^{2} / \sum A_{i} \\
I_{y y}=\frac{1}{12} \sum_{j=1}^{n}\left(x_{j} y_{j+1}-x_{j+1} y_{j}\right)\left(x_{j}{ }^{2}+x_{j} x_{j+1}+x_{j+1}{ }^{2}\right)-I_{x}{ }^{2} / \sum A_{i} \\
I_{x y}=\frac{1}{24} \sum_{j=1}^{n}\left(x_{j} y_{j+1}-x_{j+1} y_{j}\right)\left(x_{j} x_{j+1}+2 x_{j} x_{j}+2 x_{j+1} x_{j+1}+x_{j+1} x_{j}\right)- \\
I_{x} I_{y} / \sum A_{i}
\end{gathered}
$$

As the principal axes of an arbitrary polygon are those locations where the product of inertia is zero, the orientation of the principle axes with respect to the centroid and the horizontal $x$-axis can be calculated as:

$$
\theta=\frac{1}{2} \tan ^{-1}\left(2 I_{x y} /\left(I_{y y}-I_{x x}\right)\right)
$$

where $\theta$ is measured positive anticlockwise from the centroidal $x$-axis. The other principal axis is perpendicular to this one.

- Step 3: Detect the longer principal axis as the main orientation of ROI and $\omega$ is defined as the angle between the main orientation and the North direction.

To realize it, the length of principal axes within the boundary of ROI is compared and the longer one is selected. Then a vector is created pointing to the North and helping calculate the 
$\omega . \omega$ is defined in the range of $\left[-90^{\circ},+90^{\circ}\right]$ where a positive $\omega$ indicates a North-East direction and negative presents a North-West direction. Figure 7.3 provides an example where the orientation of this ROI is indicated by the solid principal axis which is longer and northnorthwest $\left(\omega=-10.4^{\circ}\right)$.

- Step 4: Detect the minimum encompassing rectangle of ROI (see Figure 7.3) which is used for defining the shape.

In this step, a shape index $s p$ is introduced to represent how elongated the shape is. It is defined as the ratio of the longer side (e.g., height) of the outside rectangle divided by the shorter side (e.g., width). The greater $s p$ is, the more elongated shape is recognised.

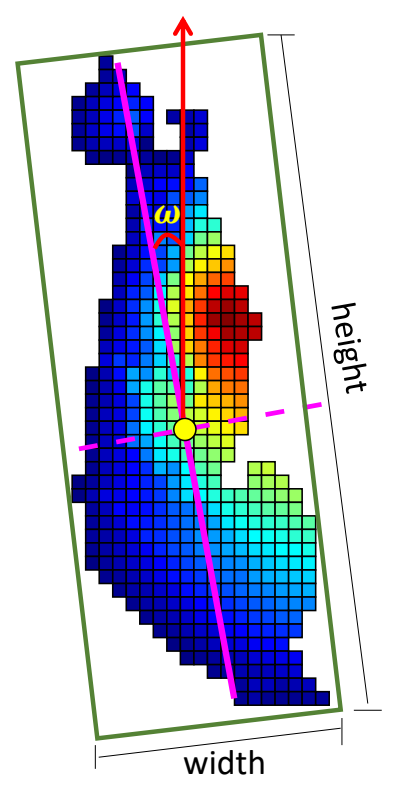

Figure 7.3 An example of ROI.

Finally, the spatial features extracted from this four-step algorithm are quantified as: 1) ROI geographic location which is represented by the coordinates of the geometric centroid $\left(C_{x}, C_{y}\right)$; 2) ROI size which is the sum of all grids of the object; 3) ROI orientation which is indicated by the angle $\omega$ with respect to the north direction and 4) ROI shape which is described by $s p$. All features are converted automatically consistent with the dataset resolution and can be saved in a text file by the toolbox. 


\subsubsection{The algorithm for quantifying the hydroclimatic features of ROI}

For a given ROI, basic features such as the areal average value and total volume of the hydroclimatic variable can be extracted straightforwardly by calculating the values of grids. To quantify the spatial distribution of the hydroclimatic variable in this ROI if it exists, the kmeans clustering technique is employed to recognise the centroid of the region(s) with the highest values which are called the "core". For example, if the dataset is grid-based hourly rainfall, the "core" with the extreme values inside the ROI can be regarded as the rainfall centre of this event whose location and the moving track can be easily monitored using the toolbox.

The k-means clustering is one of the partitioning-based techniques to divide the variables of a dataset into several non-overlapping clusters based on the degree of similarity of the variables (Friedman et al., 2001; Pham et al., 2005). To apply it, the first step is to determine the number of clusters which is usually based on the prior knowledge of the dataset. As such information is often unavailable, in this toolbox, multiple trials were carried out to find the optimal number of clusters $k$ evaluating by using the Calinski-Harabasz criterion (Caliński and Harabasz, 1974). Then the $k$-means clustering starts to partition the data by assigning $k$ initial centroids that are the farthest from one another in the data space or just by giving them random values within the space. The distance from the cluster centroids is calculated before the centroid is reassigned and updated using the mean of the centroids of the clusters. This procedure is repeated until the change of the centroid ceases. However, as the $k$-means algorithms are local search heuristics which means it is sensitive to the initial centroids (Morissette and Chartier, 2013), this step is repeated 10 times with different initial points to get a stable result and the averaged location is used as the final.

The distance between the core(s) and the ROI centroid is then calculated, as shown in Figure 7.4 where the areal average of rainfall is $29 \mathrm{~mm} /$ day, and the rainfall centre (i.e., the core) is $38 \mathrm{~mm}$ /day marked by $\times$ which sits very closed to the centroid of the ROI. 


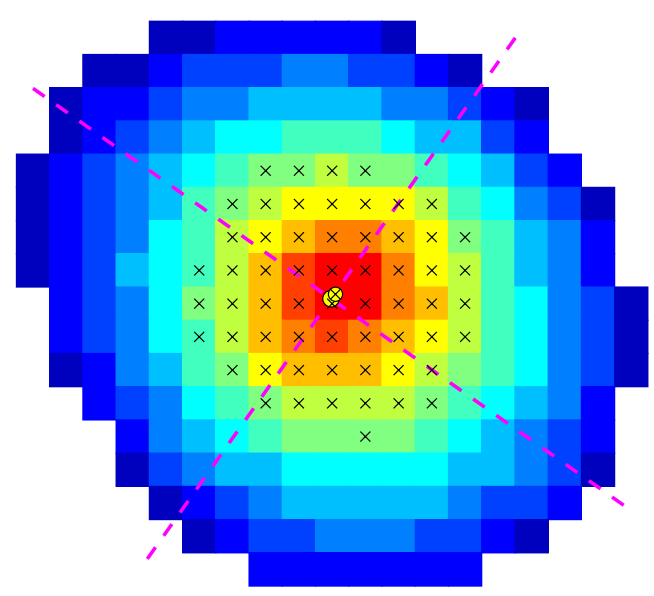

Figure 7.4 An example of ROI with its core.

\subsection{Illustrative Case Studies}

\subsubsection{Catchment-based analysis of extreme rainfall in England and Wales}

Atmospheric water, precipitation, evaporation and transpiration, and surface water are four fundamental components of the hydrological cycle. To investigate the hydrological process, research is usually carried out within a region of interest e.g., country, political boundary, river basin and even the global. The catchment is one of the basic units in quantitative hydrological analysis. In any given catchment, the runoff happens when the soil is saturated and cannot absorb the input water such as precipitation. Therefore, the area-oriented variation of rainfall is of the concern of the engineers and flood risk managers. In recent decades, many studies have focused on a catchment-based analysis involving the estimation of the volume, area, and depth of rainfall. For example, Ochoa-Rodriguez et al. (2015) investigated the impact of rainfall input resolution on the outputs of different hydrodynamic models of seven urban catchments in North-West Europe and found that increasing the catchment drainage area will decrease the impact of rainfall input resolution. Besides, many other researchers analysed gauged rainfall extremes corresponding to their hydrological response in the river catchments of different sizes (Anquetin et al., 2010; Lobligeois et al., 2014; Sangati et al., 2009). 
The SPER toolbox can be very useful in this context for supporting the variation analysis within a specific boundary due to its ability to detect the spatial characteristics of the catchment or the region of interest. Thus, the first case study demonstrates an analysis of the spatial variation of extreme rainfall in 903 catchments in England and Wales, where more details of the results are presented in Section 3.5 of Chapter 3.

\subsubsection{Pattern recognition of daily rainfall over the last century in Great Britain}

Although the distribution of rainfall is affected by many hydrometeorological variables and different topographic conditions (Kirono et al., 2010), the spatial and temporal distribution can be usually bounded within the specific patterns due to the relatively stable large-scale structure of atmospheric circulation. The SPER toolbox becomes useful to explore the spatial variation of these patterns of rainfall distribution. Therefore, to explore whether rainfall has a relatively stable pattern, in this case study, an attempt is made to use the GEAR dataset (see Table 1.1 in Chapter 1) and employ the SPER toolbox to extract the spatial features of daily rainfall pattern from the period of 1898 - 2010 in England, Wales and Scotland respectively. The identified rainfall patterns are then categorised into three classes according to their sizes, i.e., $\mathrm{C} 1$ (size $\leq$ $\left.250 \mathrm{~km}^{2}\right), \mathrm{C} 2\left(250 \mathrm{~km}^{2}<\right.$ size $\left.\leq 500 \mathrm{~km}^{2}\right)$ and $\mathrm{C} 3\left(\right.$ size $\left.>500 \mathrm{~km}^{2}\right)$. The patterns with the most frequent occurrence are recorded and summarized in Figure 7.5 followed by several specific patterns are presented in Table 7.1.

Table 7.1 Specific dominating patterns of daily rainfall in England, Wales, and Scotland catalogued at three sizes.

\begin{tabular}{|c|c|c|c|}
\hline Location & $\begin{array}{c}\mathrm{C} 1 \\
\text { size } \leq 250 \mathrm{~km}^{2}\end{array}$ & $\begin{array}{c}\mathrm{C} 2 \\
250 \mathrm{~km}^{2}<\text { size } \leq 500 \mathrm{~km}^{2}\end{array}$ & $\begin{array}{c}\mathrm{C} 3 \\
\text { size }>500 \mathrm{~km}^{2} \\
\end{array}$ \\
\hline England & $\begin{array}{l}s p: 1.0 \sim 1.2 \\
\omega: 45^{\circ} \sim 75^{\circ}\end{array}$ & $s p: 1.0 \sim 1.6$ & \\
\hline
\end{tabular}




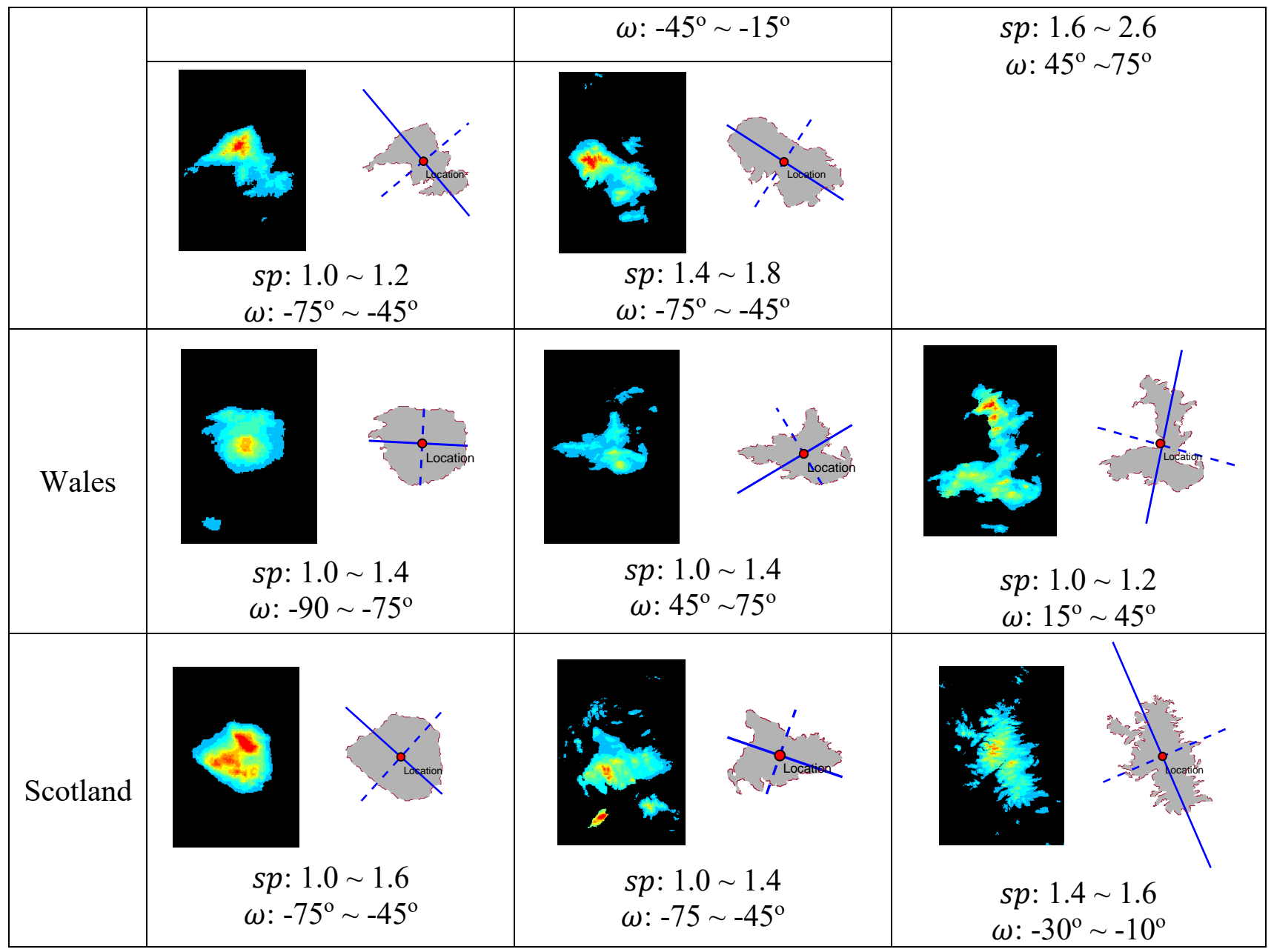

Figure 7.5 shows the number of patterns that occurred in the period of over 100 years, represented by their colour with respect to both $s p$ and $\omega$. For all three countries in general, a clear trend of decrease in the number of the identified patterns associated with large $s p$ values (indicating narrow, elongated shapes) can be seen as the size of the pattern increases (i.e., from C1 to $\mathrm{C} 3)$. 

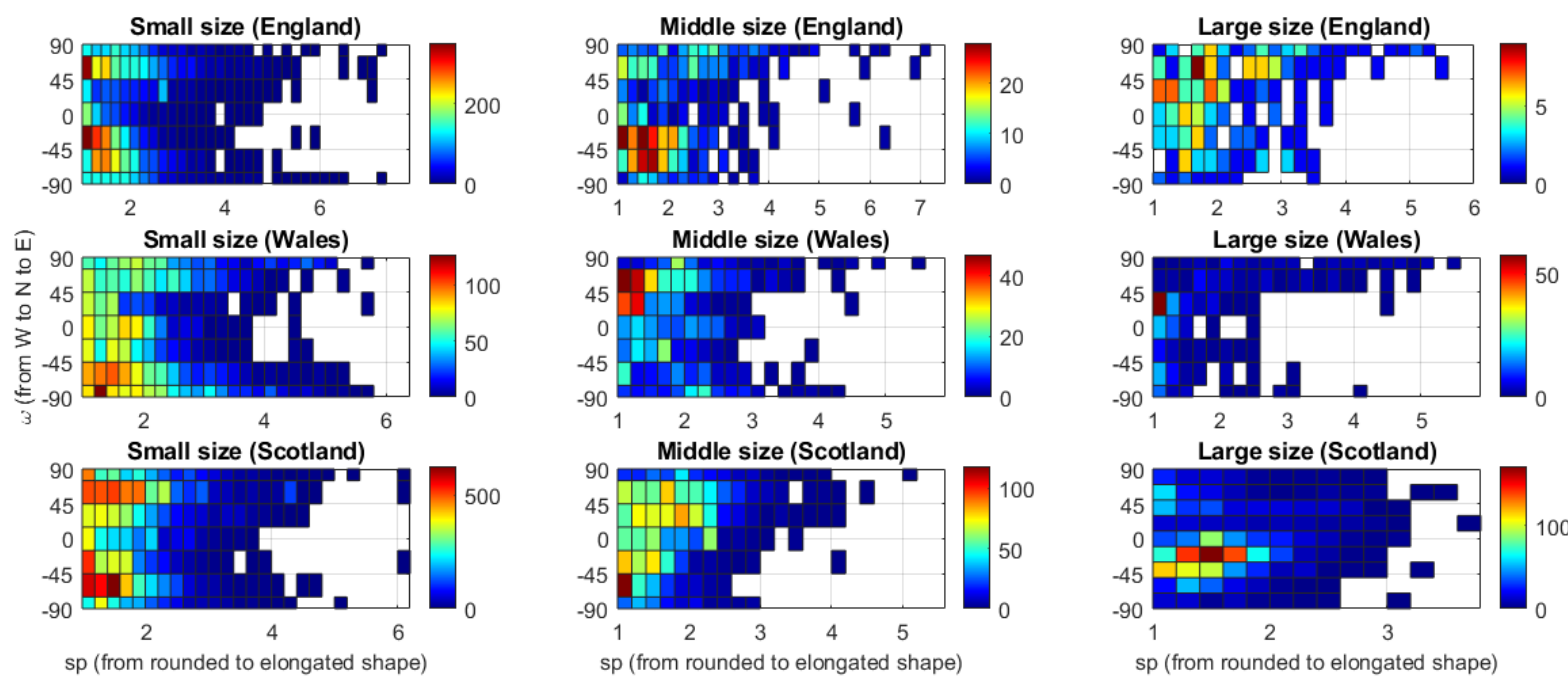

Figure 7.5 The histograms of the number of daily rainfall patterns in England, Wales and Scotland displayed by different colours with respect to $s p$ and $\omega$ at three sizes (C1, C2 and $\mathrm{C} 3)$.

In England, the most frequent rainfall patterns in $\mathrm{C} 1$ are those mainly oriented in the direction of the northeast to the east with a more rounded shape $(1.0<s p<1.2)$. However, for patterns in larger sizes (C2 and $\mathrm{C} 3$ ), the orientation of the elongation changes from mainly northwest ( $\omega$ is around $\left.-45^{\circ}\right)$ to northeast-by-east $\left(\omega \geq 45^{\circ}\right)$. This finding may be explained by the dynamics of the rainfall systems in various sizes. Small-sized rainfall distribution is likely to be driven by more localized convective storms which tend to have a more regular shape. Large rainfall systems may come as a result of frontal systems hence having a narrow band shape. Another very interesting feature is that intensive rainfall usually happened over a small region. The other two typical patterns of middle size in England are usually located in the Lake District and nearby while the ones with sizes larger than $500 \mathrm{~km}^{2}$ usually cover across the whole south of England.

In Wales, the typical pattern of less than $250 \mathrm{~km}^{2}$ category is more rounded with a slight eastwest orientation $\left(\omega \leq-75^{\circ}\right)$ while the patterns of middle size become northeast-by-eastorientated and less rounded usually with a concave in the north-west direction because of topographic effect. These patterns also have a relatively fixed location at the northwest of Cardiff near the boundary of Wales and England (location index is easting $350 \mathrm{~km}$, northing $250 \mathrm{~km}$ ). The patterns with a size larger than $500 \mathrm{~km}^{2}$ are also shown to have a very common 
"L" shape which is almost across the north and south of Wales. It should be noted that the underlying dataset is in fact 'masked' by the land boundary; and therefore, patterns of large size (C3) are artificially limited by the shape of the land.

In Scotland, the patterns whose size is smaller than $125 \mathrm{~km}^{2}$ are also more rounded and usually located north of Lake District and near the boundary of England and Scotland. The middlesized patterns whose orientation are nearly identical to those smaller rainfall patterns, have two common locations: one is to the north of Lake District while the other is in the north of Highland. The patterns with a size over $500 \mathrm{~km}^{2}$ are mainly northwest-orientated and normally cover the whole north and west of Highland.

To investigate the temporal change of rainfall patterns in the last century, the entire duration is divided into five groups. Table 7.2 lists the dominating daily rainfall pattern(s) of each group at three different sizes in England, Wales and Scotland, where the percentage in bracket presents the proportional number of patterns.

Table 7.2 Temporal change of specific rainfall patterns in three countries in three size categories.

\begin{tabular}{|c|c|c|c|c|c|c|}
\hline & & 1898-1922 & 1923-1947 & 1948-1972 & 1973-1997 & 1998-2010 \\
\hline \multirow{3}{*}{ England } & $\mathrm{C} 1$ & $\begin{array}{c}(89.2 \%) \\
s p: 1 \text { to } 1.2 \\
\omega: 45^{\circ} \text { to } 75^{\circ} \\
\end{array}$ & $\begin{array}{c}(90.6 \%) \\
s p: 1 \text { to } 1.2 \\
\omega:-45^{\circ} \text { to }-15^{\circ}\end{array}$ & $\begin{array}{c}(89 \%) \\
s p: 1.2 \text { to } 1.4 \\
\omega:-45^{\circ} \text { to }-15^{\circ}\end{array}$ & $\begin{array}{c}(89 \%) \\
s p: 1.2 \text { to } 1.4 \\
\omega:-45^{\circ} \text { to }-15^{\circ}\end{array}$ & $\begin{array}{c}(88.9 \%) \\
s p: 1.2 \text { to } 1.4 \\
\omega:-45^{\circ} \text { to }-15^{\circ}\end{array}$ \\
\hline & $\mathrm{C} 2$ & $\begin{array}{c}(8 \%) \\
s p: 1.6 \text { to } 1.8 \\
\omega:-75^{\circ} \text { to }-45^{\circ} \\
s p: 1 \text { to } 1.2 \\
\omega:-45^{\circ} \text { to }-15^{\circ}\end{array}$ & $\begin{array}{c}(7.8 \%) \\
s p: 1.4 \text { to } 1.6 \\
\omega:-45^{\circ} \text { to }-15^{\circ}\end{array}$ & $\begin{array}{c}(7.9 \%) \\
s p: 1 \text { to } 1.4 \\
\omega:-75^{\circ} \text { to }-15^{\circ}\end{array}$ & $\begin{array}{c}(8 \%) \\
s p: 1.8 \text { to } 2.0 \\
\omega:-75^{\circ} \text { to }-45^{\circ} \\
s p: 1.0 \text { to } 1.2 \\
\omega:-45^{\circ} \text { to }-15^{\circ}\end{array}$ & $\begin{array}{c}(7.9 \%) \\
s p: 1.6 \text { to } 1.8 \\
\omega:-45^{\circ} \text { to }-15^{\circ}\end{array}$ \\
\hline & $\mathrm{C} 3$ & $\begin{array}{c}(2.8 \%) \\
s p: 2.4 \text { to } 2.6 \\
\omega: 45^{\circ} \text { to } 75^{\circ}\end{array}$ & $\begin{array}{c}(1.6 \%) \\
s p: 1 \text { to } 2.8 \\
\omega:-15^{\circ} \text { to } 75^{\circ} \\
\end{array}$ & $\begin{array}{c}(3.1 \%) \\
s p: 1.6 \text { to } 1.8 \\
\omega: 45^{\circ} \text { to } 75^{\circ}\end{array}$ & $\begin{array}{c}(3 \%) \\
s p: 1 \text { to } 1.2 \\
\omega: 15^{\circ} \text { to } 45^{\circ}\end{array}$ & $\begin{array}{c}(3.2 \%) \\
s p: 1.4 \text { to } 1.6 \\
\omega:-45^{\circ} \text { to }-15^{\circ}\end{array}$ \\
\hline \multirow[t]{2}{*}{ Wales } & $\mathrm{C} 1$ & $\begin{array}{c}(77.2 \%) \\
s p: 1.2 \text { to } 1.4 \\
\omega:-90 \text { to }-75^{\circ}\end{array}$ & $\begin{array}{c}(80 \%) \\
s p: 1.2 \text { to } 1.4 \\
\omega:-90 \text { to }-75^{\circ}\end{array}$ & $\begin{array}{c}(79.8 \%) \\
s p: 1.4 \text { to } 2.0 \\
\omega:-90 \text { to }-45^{\circ}\end{array}$ & $\begin{array}{c}(80.3 \%) \\
s p: 1.8 \text { to } 2.0 \\
\omega:-90^{\circ} \text { to }-75^{\circ} \\
s p: 1.4 \text { to } 1.6 \\
\omega:-45^{\circ} \text { to }-15^{\circ}\end{array}$ & $\begin{array}{c}(80.6 \%) \\
s p: 2 \text { to } 2.2 \\
\omega:-75^{\circ} \text { to }-45^{\circ}\end{array}$ \\
\hline & $\mathrm{C} 2$ & $\begin{array}{c}(17.6 \%) \\
s p: 1 \text { to } 1.2 \\
\omega: 45^{\circ} \text { to } 75^{\circ}\end{array}$ & $\begin{array}{c}(16.2 \%) \\
s p: 1.2 \text { to } 1.4 \\
\omega: 45^{\circ} \text { to } 75^{\circ}\end{array}$ & $\begin{array}{c}(13.7 \%) \\
s p: 1.2 \text { to } 1.4 \\
\omega: 15^{\circ} \text { to } 45^{\circ}\end{array}$ & $\begin{array}{c}(13.8 \%) \\
s p: 1.6 \text { to } 1.8 \\
\omega: 45^{\circ} \text { to } 75^{\circ}\end{array}$ & $\begin{array}{c}(14 \%) \\
s p: 1 \text { to } 1.2 \\
\omega: 15^{\circ} \text { to } 45^{\circ}\end{array}$ \\
\hline
\end{tabular}




\begin{tabular}{|c|c|c|c|c|c|c|}
\hline & & & & $\begin{array}{l}s p: 2.2 \text { to } 2.4 \\
\omega:-90 \text { to }-75^{\circ}\end{array}$ & & \\
\hline & $\mathrm{C} 3$ & $\begin{array}{c}(5.2 \%) \\
s p: 1 \text { to } 1.2 \\
\omega: 15^{\circ} \text { to } 45^{\circ}\end{array}$ & $\begin{array}{c}(5.8 \%) \\
s p: 1 \text { to } 1.2 \\
\omega: 15^{\circ} \text { to } 45^{\circ}\end{array}$ & $\begin{array}{c}(6.5 \%) \\
s p: 1 \text { to } 1.2 \\
\omega: 15^{\circ} \text { to } 45^{\circ}\end{array}$ & $\begin{array}{c}(5.9 \%) \\
s p: 1 \text { to } 1.2 \\
\omega: 15^{\circ} \text { to } 45^{\circ}\end{array}$ & $\begin{array}{c}(5.4 \%) \\
s p: 1 \text { to } 1.2 \\
\omega:-75^{\circ} \text { to }-45^{\circ} \\
s p: 1 \text { to } 1.2 \\
\omega:-15^{\circ} \text { to } 45^{\circ}\end{array}$ \\
\hline \multirow{3}{*}{ Scotland } & $\mathrm{C} 1$ & $\begin{array}{c}(74.2 \%) \\
s p: 1 \text { to } 1.2 \\
\omega: 75^{\circ} \text { to } 90^{\circ}\end{array}$ & $\begin{array}{c}(76.8 \%) \\
s p: 1.2 \text { to } 1.4 \\
\omega:-75^{\circ} \text { to }-45^{\circ}\end{array}$ & $\begin{array}{c}(83.8 \%) \\
s p: 1.4 \text { to } 1.6 \\
\omega:-75^{\circ} \text { to }-45^{\circ}\end{array}$ & $\begin{array}{c}(83.8 \%) \\
s p: 1.4 \text { to } 1.6 \\
\omega:-75^{\circ} \text { to }-45^{\circ}\end{array}$ & $\begin{array}{c}(83.8 \%) \\
s p: 1.2 \text { to } 1.4 \\
\omega: 45^{\circ} \text { to } 75^{\circ}\end{array}$ \\
\hline & $\mathrm{C} 2$ & $\begin{array}{c}(12.6 \%) \\
s p: 1 \text { to } 1.2 \\
\omega:-75^{\circ} \text { to }-45^{\circ}\end{array}$ & $\begin{array}{c}(11.9 \%) \\
s p: 1 \text { to } 1.2 \\
\omega:-75^{\circ} \text { to }-45^{\circ}\end{array}$ & $\begin{array}{c}(8.9 \%) \\
s p: 1 \text { to } 1.2 \\
\omega:-45^{\circ} \text { to }-15^{\circ} \\
\end{array}$ & $\begin{array}{c}(8.8 \%) \\
s p: 1.4 \text { to } 1.6 \\
\omega:-45^{\circ} \text { to }-15^{\circ} \\
\end{array}$ & $\begin{array}{c}(8.9 \%) \\
s p: 1.4 \text { to } 1.6 \\
\omega:-45^{\circ} \text { to }-15^{\circ} \\
\end{array}$ \\
\hline & C3 & $\begin{array}{c}(13.2 \%) \\
s p: 1.2 \text { to } 1.4 \\
\omega:-60^{\circ} \text { to }-30^{\circ}\end{array}$ & $\begin{array}{c}(11.3 \%) \\
s p: 1.5 \text { to } 1.8 \\
\omega:-30^{\circ} \text { to } 0^{\circ}\end{array}$ & $\begin{array}{c}(7.3 \%) \\
s p: 1.5 \text { to } 1.8 \\
\omega:-30^{\circ} \text { to } 0^{\circ}\end{array}$ & $\begin{array}{c}(7.4 \%) \\
s p: 1.2 \text { to } 1.5 \\
\omega:-30^{\circ} \text { to } 0^{\circ}\end{array}$ & $\begin{array}{c}(7.3 \%) \\
s p: 1.2 \text { to } 1.8 \\
\omega:-30^{\circ} \text { to } 0^{\circ}\end{array}$ \\
\hline
\end{tabular}

In England, the percentage of the patterns in $\mathrm{C} 2$ keeps stable while that for $\mathrm{C} 1$ decreases and C3 increases after 1947. For the patterns in C1, their shapes change slightly from a more rounded shape to an ellipse and the orientation shifts from northeast-by-east to northwest-bynorth after 1922. For the patterns of the C2 category, both their shapes and orientations are stable and in the range of 1 to 1.8 and northwest respectively. For the patterns in $\mathrm{C} 3$, their shapes are more elongated and the orientation changes from northeast-by-east to northwest after 1997.

In Wales, a very different temporal variation has been detected. First, the fractions of the patterns in different size categories remain almost unchanged. Second, for individual size group, the patterns in $\mathrm{C} 1$ become more and more elongated while their main orientation remains northwest-by-west; the patterns in C2 do not change much in their elongated shape, but their orientation shifts from northeast-by-east to northeast-by-north; For the patterns in the C3 category, they all have a similar shape and orientation as shown in Table 7.1, and do not change over time. Again, the boundary impact mentioned previously may be the cause of the behaviour.

In Scotland, the situation of percentage is just opposite to what has been shown in England. The percentage of the patterns in $\mathrm{C} 1$ increases while those of $\mathrm{C} 2$ and $\mathrm{C} 3$ both decrease 
especially after the year 1947. The shape of the patterns in Clvaries from a more rounded one to rectangular, which is similar to that of England but the orientation changes from northwestby-west to northeast-by east. For the patterns in $\mathrm{C} 2$, their shapes appear to be more and more elongated and the orientation shifts from northwest-by-west to northwest-by-north after 1947. Like in Wales, the shape and orientation of the patterns in $\mathrm{C} 3$ are almost unchanged during the time.

\subsubsection{Tracing rainfall area and spatial distribution in 24 hours in Guangzhou, China}

Rainfall is one of the basic components of the hydrological circle where many studies focused on analysing the duration, intensity, and spatiotemporal variation of rainfall to enhance the understanding of its interaction with the hydrological process. To monitor a rainfall event, the traditional approach makes use of rain gauge measures to calculate the rainfall intensity and cumulative amount for characterising the rainfall behaviour (Muthusamy et al., 2017a; Salio et al., 2015; Sohn et al., 2010). However, the variability of rainfall is difficult to detect by a limited number of rain gauges and the measurement affected by many factors such as wind, manual error, etc. can somehow cause uncertainties even errors. In recent decades, with the rapid developments in environmental monitoring technology, weather radars with their high spatial and temporal resolution have been widely applied to monitor and measure rainfall (Kidd and Huffman, 2011). The spatial and temporal patterns collected by weather radars are further used to model the rainfall event and the variability detected from the consecutive series of patterns can be used for forecasting. Therefore, identifying and tracking the rainfall events is an essential task for radar-based hydrometeorological applications and how to process it accurately and automatically is still a big challenge (Sokol et al., 2021). Nowadays, there are two types of algorithms for identifying and tracking rainfall patterns: 1) the pixel-based algorithm which makes use of pixel information and extracts the motion vectors by searching the maximum correlation coefficient of rainfall pattern in two consecutive radar images; 2) the object-based algorithm which makes use of the properties of the discrete rainfall pattern such as centroid, area, etc., in consecutive radar images (He et al., 2019). However, both algorithms 
have drawbacks, e.g., the pixel-based algorithm is easily affected by the noise and the motion estimates solely based on the maximum correlation coefficient may produce inconsistent classification results while the object-based algorithm is not good at estimating motions especially when the shape of rainfall pattern change rapidly.

Therefore, a more stable algorithm for quantifying and classifying the rainfall pattern is required. As the SPER toolbox provides a stable calculation for quantifying the spatial and hydrological features of rainfall patterns, this study employs the SPER toolbox to monitor the rainfall area and trace the rainfall centres. The data applied to the study are collected by the cutting-edge active phase array radar (APRA) which are one of the latest developments of radar rainfall measurements with very high spatial-temporal resolution (e.g., 30 metres and 30 seconds) and is suitable for small to medium, built-up areas. The advances of APARs lies in not only their ability of much-refined precipitation distributions, but their potential to be a realtime driver for probabilistic flood forecasting and early warning systems. In this case, the data of rainfall on 15 April 2019 in Guangzhou, China are accumulated with the resolution of 5 minutes/60 metres and the SPER toolbox are used to detect the rainfall area which is defined within a closed boundary and the rainfall centre for each area.

The top left panel of Figure 7.6 presents the rainfall at 00:00 which is the start of radar measurement and red dots mark the rainfall centres (i.e., the core defined in Figure 7.4). Then the outer boundary of these rainfall centres is detected in order to demonstrate how the rainfall is centralised over the study area. The rest figures of Figure 7.6 show the changes of the scattered area of rainfall centres over space where the scattered area of rainfall in the first three hours shrinks and moves southward, then the rainfall decreases in the south however the north region starts to rain and the scattered area of rainfall centres is increased in the following three hours. From 06:00 to 09:00 the scattered area moves eastward and reduces after an increase around 08:00. There is no rainfall in the next several hours and rainfall starts from 13:30 and the scattered area is northwest-orientated and located in the East region. Then rainfall moves to the southwest and the scattered area shrinks during 15:00 and 18:00. In the following three hours, the scattered area keeps relative still after the rainfall centres move north. In the same 
regions, the scattered area of rainfall in the last three hours is finally centralised in the east (i.e., $0 \sim 1000$ and 500 2000).
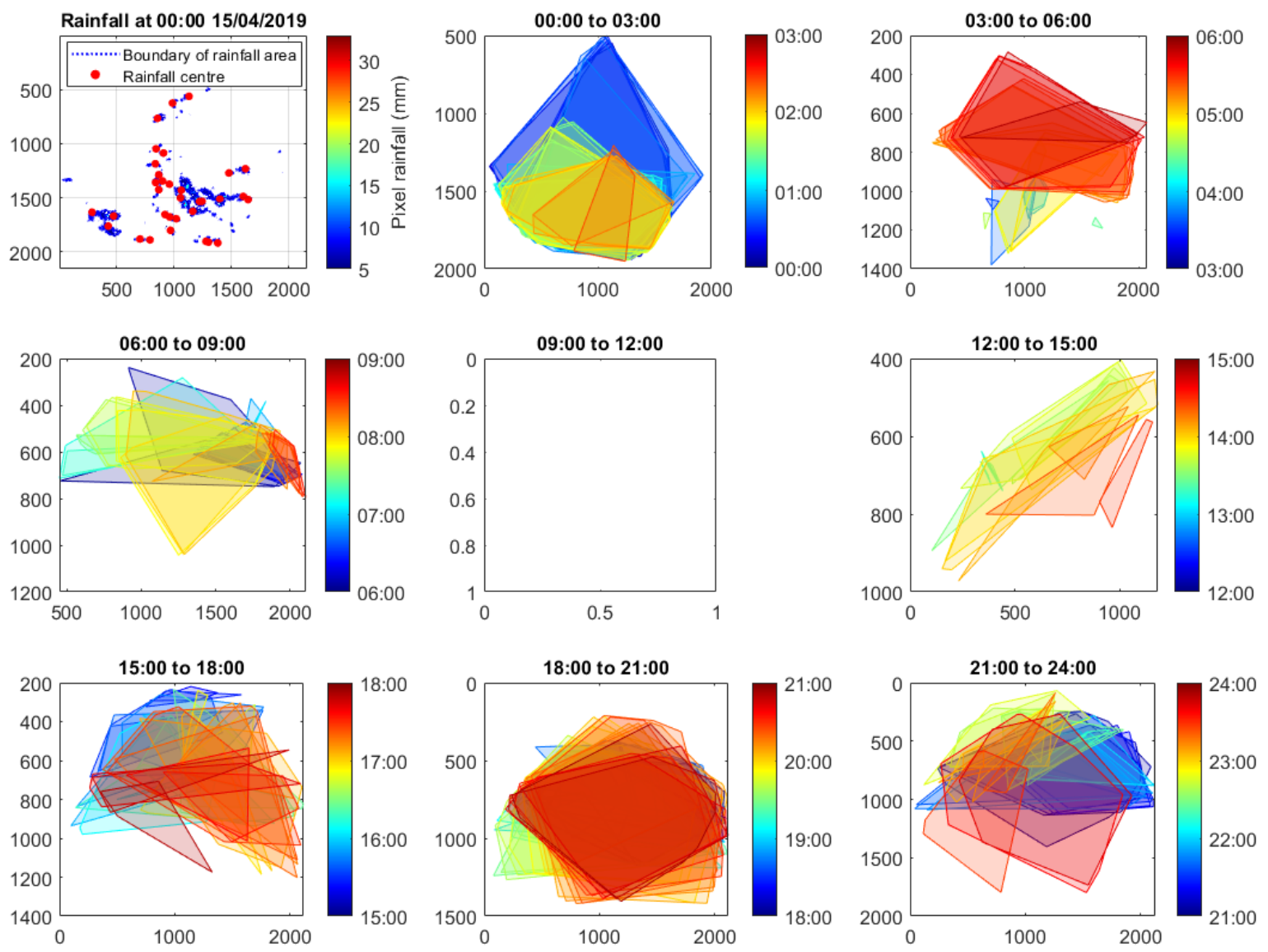

Figure 7.6 The changes of the scattered area of rainfall centres in 24 hours.

Figure 7.7 focuses on the largest rainfall area and presents its characteristics such as the areal average rainfall, the rainfall at its centre and the orientation, and the moving track. It can be observed that the areal rainfall in the early nine hours is generally lower than the last nine hours but the highest rainfall in the rainfall centre is in 06:00-07:00 when the areal rainfall is not very high. As for the area orientation, the majority shows east-west orientated or northwest-by-west or northeast-by-east, i.e., $\omega$ is near $\pm 90^{\circ}$, and the north-south orientation is rare in the 24 hours. Besides, Figure 7.7b shows the moving track of the largest rainfall area clearly and colour indicates the time, which can be specified as: southeast $\rightarrow$ northwest $\rightarrow$ east $\rightarrow$ southwest $\rightarrow$ east $\rightarrow$ north. 

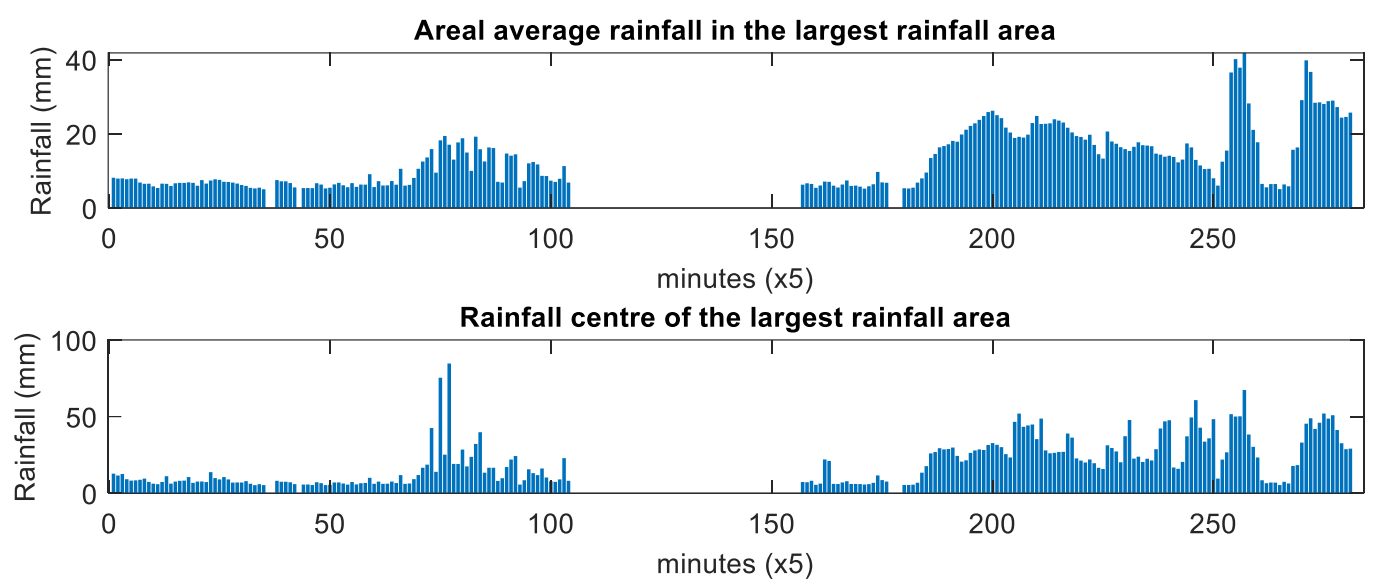

Orientation of the largest rainfall area

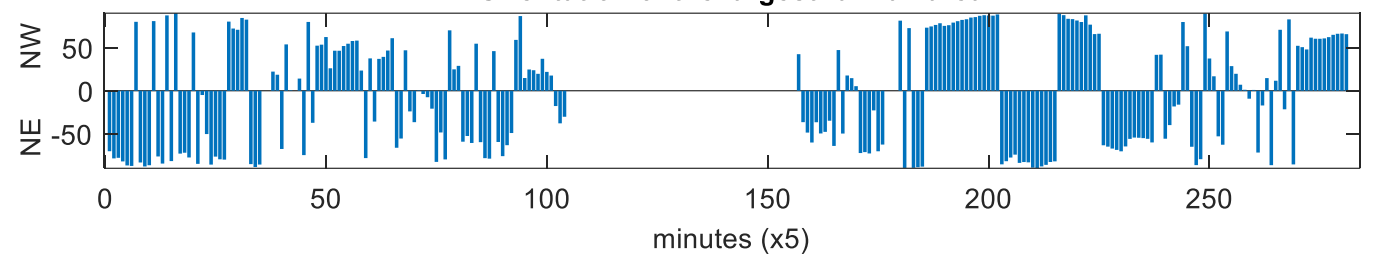

(a)

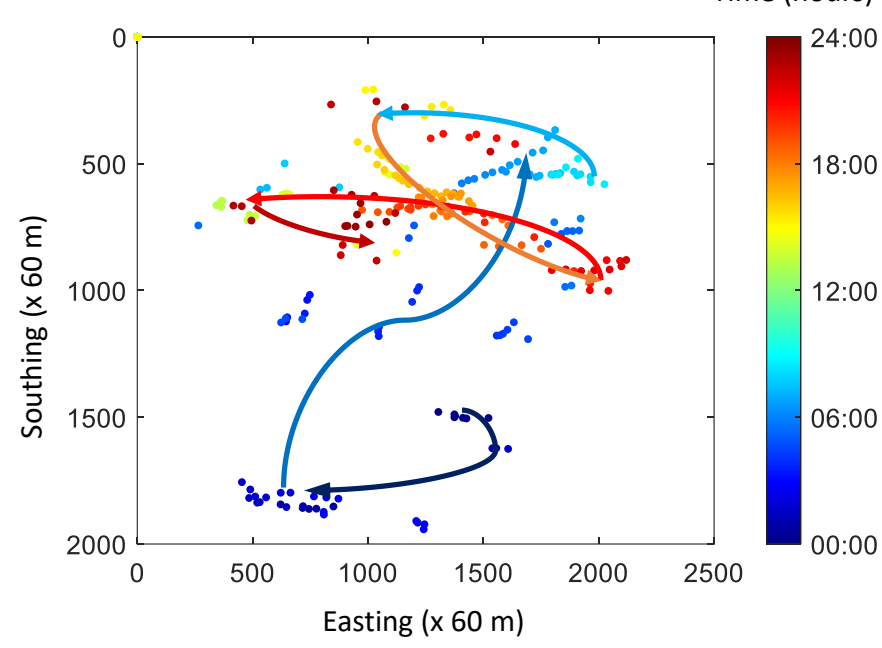

(b)

Figure 7.7 Characteristics of the largest rainfall area (a) and its moving track (b) in 24 hours. 


\subsection{The front-end application for training a convolution neural network}

The new integrated classification capacity of the SPER toolbox makes it possible to automatically produce labelled training sets from tens of thousands of large-scale environmental images for deep-learning applications in feature recognition and forecasting. In this process, the attributes such as those spatial features (location, size and orientation) can be quickly identified to pre-categorise the data or images into different labels/classes. To demonstrate this, an Alexnet model (Alom et al., 2018) of convolution neural network (CNN) is built to demonstrate the process of auto-identifying the daily rainfall patterns in GB, using the training sets auto-labelled by the SPER toolbox. There are four labels used in this test, i.e., no rainfall (L0), concentric pattern (L1), elongated pattern (L2) and compound pattern (L3), as shown in Figure 7.8a.
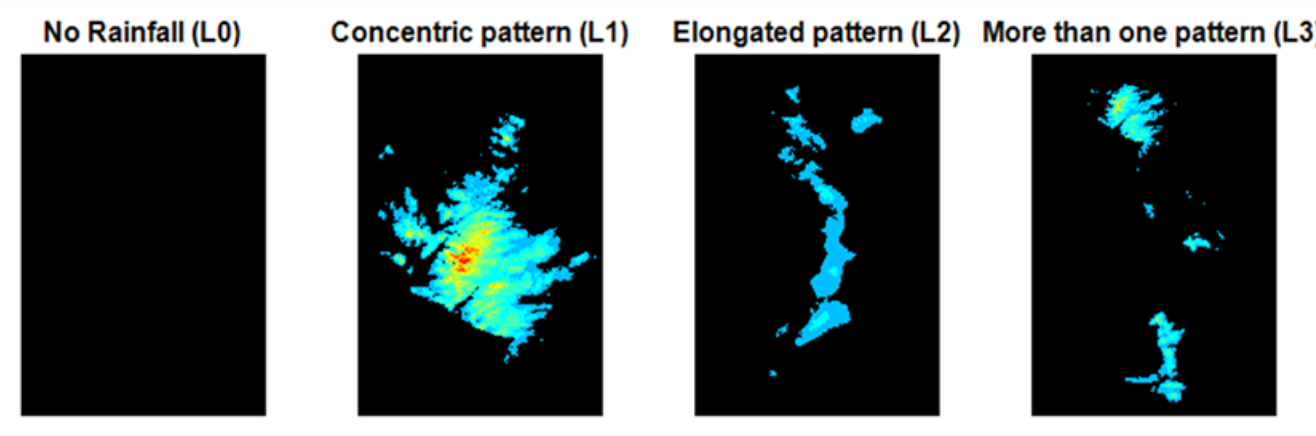

No Attribute (L0)

Attribute for L1

Attribute for L2

Attribute for L3
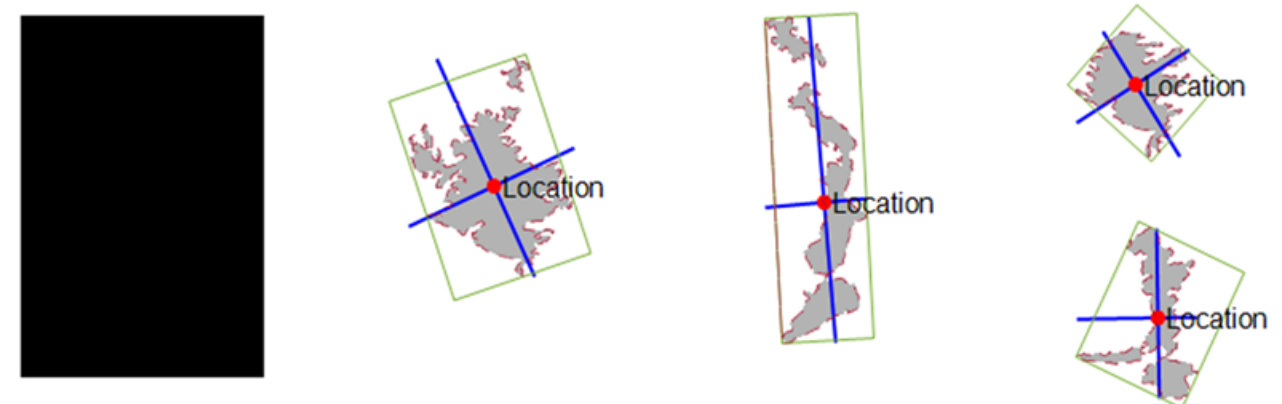

(a) 


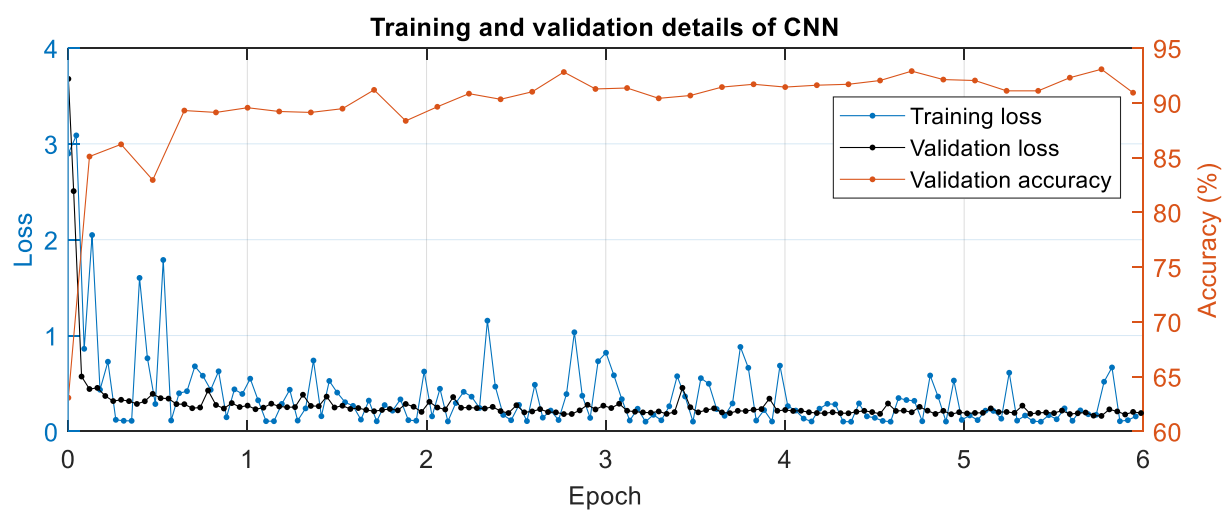

(b)

Figure 7.8 Four labels corresponding to the daily rainfall patterns: no rainfall (L0), concentric pattern (L1) where its sp is near 1.0, elongated pattern (L2) and compound pattern (L3) which has both concentric and elongated types (a); and the training and validation loss (blue and black lines) and validation accuracy (orange lines) of $\mathrm{CNN}(\mathrm{b})$.

To address the issue of the overhead demand for computation (e.g., more than 40 thousand daily rainfall images), Alexnet (which is a type of CNNs) was constructed for training because it reduces the overfitting and allows for multi-GPU training which reduces the training time (Krizhevsky et al., 2012). There are eight layers in the Alexnet model, with the first five being convolutional layers having the kernel sizes of $11 \times 11,5 \times 5$ and $3 \times 3$ and the last three fully connected layers. The structure is designed as: the first convolutional layer $(11 \times 11)$ followed by an overlapping max pooling layer is connected to the second convolutional layer $(5 \times 5)$ which is also followed by one overlapping max pooling layer; then the rest three convolutional layers $(3 \times 3)$ are connected directly and the end links to one overlapping max pooling layer; and the final link is to the last three fully connected layers. ReLU (Pedamonti, 2018), a nonlinear activation function, is applied after all the convolution and fully connected layers to get activation values corresponding to neurons. To apply the Alexnet model, firstly all images were converted to the acceptable size which is $227-$ by-227-by-3 where the last dimension size 3 indicates the three colour channels (e.g., R, G, B). Then the last three layers were modified to specify 4 classes for classification and set a faster learning rate for these newly modified layers and a slower learning rate for the transferred layers in order to obtain an effective and better transfer learning. Finally, 1166 daily rainfall images are randomly selected from all images while the rest are used for training the Alexnet model. 
During the training phase, the Alexnet of CNN model learns the training sets and selects $30 \%$ for self-validation randomly and the model validates the network every 3 iterations. The testing sets, i.e., 1166 images, are applied for predicting their classifications. The track of training and validation results are shown in Figure $7.8 \mathrm{~b}$ where the training loss and the validation accuracy vary dramatically during the first epoch with 680 iterations then gradually converge to 0.17 and $92.5 \%$. The toolbox has been shown to reach a test accuracy of $93.4 \%$ in classification, i.e., with 1089 out of 1166 new rainfall images having been classified correctly. However, the inherent complexities of the environmental data cause complicated patterns as well. Further work is recommended to make the toolbox more robust to process climate projection data at various, often low resolutions. A downscaling or down-sampling method may need to be integrated. Certainly, optimising the $\mathrm{CNN}$ algorithm should be carried out for recognising more complex patterns other than the simple example shown in this chapter.

\subsection{Summary and remarks}

This chapter discusses the development of a spatial pattern extraction and recognition (SPER) toolbox for automatic extracting and classifying extreme hydroclimatic patterns by their spatial features i.e., location, size, orientation and shape, and hydroclimatic features if the input is gridbased datasets. The main aims of designing the toolbox are 1) to develop a stable algorithm for automatically identifying and classifying the spatial features that are linked to hydroclimatic extremes; 2) to be used as a frontend that supports AI-based training in tracking and forecasting extremes; and 3) to support short-term nowcasting of extreme rainfall.

Three example application cases are given in which the implementation details are discussed. The first application shows that with this toolbox, spatial characteristics of river catchments can be easily quantified and compiled, which can support any similar catchment-based analysis; the second application shows that the toolbox can help classify the variation patterns of extreme rainfall and top 3 dominating rainfall patterns in England, Wales and Scotland and how they change over the last century are detected; the third application presents the track of rainfall area and its spatial variation in 24 hours in Guangdong, China. Its potential application 
and its link to deep/machine learning are also presented where the toolbox was used for precatalogued the hydroclimatic patterns from the dataset into several different labels which are used for training a supervised artificial neural network (e.g., convolution neural network is used in this study).

The source code of the SPER toolbox subject to a GPL V3 licence is available at GitHub (https://github.com/wanghan924/SPER-toolbox). Use/fork of the toolbox is subject to proper acknowledgement as stated on the Webpage of the toolbox. 



\section{Part III}

Decision making under uncertainty on hydroclimatic extremes 



\section{Chapter 8 Decision-making under Uncertainty in the Context of Flood Forecasting and Early}

\section{Warning}

From the previous chapters which focus on the quantitative modelling of both univariate and multivariate hydroclimatic extremes under climate change, it is clear that not only data and models, but also the nature of decision-makers can increase the complexity of the process of decision making in managing the risks arising from hydroclimatic extremes. To improve the understanding of decision-making under uncertainty in the context of flood forecasting and early warning and to address the research question Q7, this chapter demonstrates a study on modelling the decision making process using the response data from a real-life experiment ${ }^{8}$ where 168 professionals specialised in water management were confronted with a series of binary decision problems based on probabilistic flood forecasts in different contexts. Two existing decision modelling models from unrelated disciplines, namely the Cumulative Prospect Theory (CPT) from the field of psychology, and the Decision Trees (DT) from the discipline of Machine Learning, are refined and modified to simulate the process of decision making. The resultant models are further tested on another decision-making game proposed by Ramos et al. (2013).

\footnotetext{
${ }^{8}$ It should be noted that this experiment was carried out and the two decision making models were built in the year of 2016-2017 before I started my Ph.D. research.
} 


\subsection{Introduction}

The process of translating flood hazard warnings into effective decisions usually follows a pathway that includes data collection, modelling, data assimilation, and decision-making (Jha et al., 2012). Each step has associated uncertainties that not only are difficult to quantify, but often comes with interacted effects; therefore, the decision-making process carried out at the end is complex (Arabameri et al., 2019; Beven and Alcock, 2012). During the last few decades, many efforts have been focused on improving meteorological and hydrologic prediction models and methods to quantify the uncertainty, e.g., Her et al. (2019), Qi et al. (2016), Meresa and Romanowicz (2017), and how to reduce it (Clark et al., 2016; Haghnegahdar et al., 2017; Lehner et al., 2019; Parkes and Demeritt, 2016). However, the risk associated with environmental hazards such as floods not only depends on the uncertainties coming from the data and the modelling exercise itself, but also the behaviour of decision-makers who make use of them, as well as the decision-making conditions and cultures, and the communication between modellers and decision-makers who take responsibilities (IRDR, 2013; ISCU, 2008). Understanding human-driven decision-making processes in managing risks arising from extreme events such as floods are very crucial in the effort of loss reduction and mitigating the damage to properties.

There have been two mainly used approaches when it comes to modelling the decision-making process. The first approach aims to integrate human behaviour dynamics into the decisionmaking process. For example, Aerts et al. (2018) proposed to integrate the societal behaviour and behavioural adaptation dynamics into flood risk quantifications, which leads to a more accurate characterization of flood risks. Bodoque et al. (2019) integrated risk communication into flood risk management which shows an increase in flood risk perception and awareness. Chan and Song (2010) incorporated the feedback and reward mechanisms into the decisionmaking process to improve the understanding of the decision support system. The second approach aims to understand the human-driven factors that affect the decision-making process. One of the most influential works in this field is the Cumulative Prospect Theory (CPT), proposed in the seminal paper of Tversky and Kahneman (1992), which provided the 
foundation to understand decision making under uncertainty when decisions involving monetary losses and gains. This paper, referred hereafter as KT92, is a modification to their original Prospect Theory (Kahneman and Tversky, 1979), which shows that, in general, people: 1) have different risk attitudes (generally classified as risk-averse, risk-neutral and riskseeking) depending on how the decision-making problems are formulated; 2) care more about potential losses than potential gains, and 3 ) tend to the over-weigh extreme but improbable events and under-weigh common events.

From the perspective of water resources management, Alfonso and Price (2012) applied the concept of Value of Information to indirectly incorporate the characteristics of the decisionmakers and analyse its effects for the design of water stage monitoring networks and further supporting the use of probabilistic flood maps (Alfonso et al., 2016). Many factors driven by human behaviour have also been explored in the context of flood risk management, such as risk perception (Buchecker et al., 2013; Quinn et al., 2019), risk presentation e.g. the effect of including information about uncertainty (Joslyn and LeClerc, 2012) and the ways to communicating uncertain information (Mulder et al., 2020). In particular, Ramos et al. (2013) analysed how the addition of flood forecast uncertainty information influenced decisionmaking in the context of operational flood forecasting, early warning, and control; they designed a decision-making game called "Do probabilistic forecasts lead to better decisions?" and concluded that in the absence of uncertainty information, decision-makers are compelled towards a more risk-averse attitude. Several instances of this game are used in this chapter.

Yet there is another approach to explore decision-making, which is through the use of datadriven techniques such as Machine Learning. For example, Decision Trees (DT; Mitchell, 1997; Quinlan, 1990) are widely applied in flood early warning (Costache, 2019; Tehrany et al., 2013), water quality in rivers (Atkins et al., 2007; Saghebian et al., 2014; Vélez et al., 2014), landslides (Hong et al., 2018; Tsangaratos and Ilia, 2016) and hydraulic models (Pappenberger et al., 2006).

Although the aforementioned studies, as well as other related researches (Coughlan de Perez et al., 2016; Todini, 2017), have contributed to the understanding of the decision-making under 
uncertainty, several aspects still need to be understood, particularly in the context of flood forecasting early warning (FFEW): 1) to understand for which ranges of the probability of flood occurrence, the changes of risk attitudes lead to a change in a decision related to flood evacuation; 2) to understand to what extent the a-priori knowledge of the consequences of decisions influences final decisions and if the formulation of the decision-making problem has some effect; 3) to understand to what extent the decision models can be built to predict decisions based on observations such as probabilistic forecasts of flood events, different consequences of decisions and different natures of human.

To address these purposes, in this chapter, two decision models (CPT and DT models) are reconstructed and improved using the dataset collected from a survey-based experiment where several sets of one-time binary decision-making problems were formulated. The problems were designed in a way that they resemble those typically faced by a decision-maker when making flood risk management related decisions, e.g., probability of flood forecast and monetary (and non-monetary) consequences of the decisions, plus other background information. A group of 186 participants were involved to solve these decision problems before the CPT model was built on the outcome of the experiment, using the CPT concepts established by KT92. In addition, another DT model was developed using the same data. Both models were used to analyse how the decisions of the majority are affected by the probabilities and costs of the occurrences of extreme events (in this case, flood). The two models, though totally different in nature, were further used to analyse different risk attitudes (i.e., risk aversion, risk neutrality and risk seeking). Compared with the previous CPT and DT models, the new CPT model is greatly improved on estimating the parameters and the new DT model works more effective by refining the attributes. The usability of both new models was validated by feeding them with another dataset which was collected during a period of 6 years and obtained during the execution of the decision-making experiment presented in Ramos et al. (2013) where a different group of 145 participants were involved. The results of the model on predicting/simulating decisions in the context of FFEW were discussed and compared with the traditional cost/loss model widely applied in the FFEW system. 
The remainder of this chapter is organised as follows: first, a brief introduction to decisionmaking experiment design and implementation, two old CPT and DT models are provided in this section. It should be noted that this work (designing and executing the experiment, building CPT and DT models) has been done before my Ph.D. research. Then the method of reconstructing CPT and DT models is presented in Section 8.2. Section 8.3 shows the results and performance of the two models. Next, the new models are tested and further applied with the data collected by the experiment of Ramos et al. (2013) presented in Section 8.4. The last Section 8.5 is dedicated to conclusions.

\subsubsection{Experiment design and implement}

The experiment was designed and processed to collect the decisions for building and training the decision models and it has been carried out during my MSc study where the data are further used in this chapter. This section gives a brief introduction of the setup and process of the experiment and more details can be checked in my MSc thesis (Wang, 2017).

The experimental setup simulates the working principles of a flood forecasting, warning and response system (FFWRS). The decision-making problem is framed as a decision-maker who is responsible for managing a flood-prone region, populated by a known number of residents, with estimated flood-related losses. The region has a flood warning system in place, which provides the probability of flood occurrence $p$. The decision-maker is then confronted with a situation of deciding between issuing a warning to the public or not. Either decision has associated consequences, depending on whether the flood actually occurs. If the decisionmaker decides to issue a warning, the decision will a fixed associated $\operatorname{cost}\left(C_{f}\right)$, irrespective of whether the predicted flood occurs or not. If a flood does happen, the potential unavoidable flood damage $\left(L_{u}\right)$ is added to the consequences of the decision, and therefore the associated consequence in this situation is $C_{11}=C_{f}+L_{u}$. However, if the flood does not happen, the related intangible cost $(f)$ due to disturbances to the public is added instead, and therefore the associated consequences are $C_{12}=C_{f}+f$. The opposite case is: if the decision-maker decides not to issue a warning and flood occurs, the consequence of the decision is the total flood 
damage cost, which is the sum of the avoidable flood damage $\left(L_{a}\right)$ and the potential unavoidable flood damage $\left(L_{u}\right)$, so $C_{21}=L_{a}+L_{u}$. However, if the flood does not occur, the consequences can be assumed as $C_{22}=0$. Therefore, the basic decision-making problem is formulated as follows:

"Flood is expected in your area with a probability of occurrence $p$. You have to decide among two prospects:

$P_{y}$ : If you decide to issue a warning $\left(D_{y}\right)$, there are two situations: flood occurs, you must pay $C_{11}=C_{f}+L_{u}$ tokens; flood doesn't occur, you must pay $C_{12}=C_{f}+f$ tokens.

$P_{n}$ : If you decide not to issue a warning $\left(D_{n}\right)$, there are two situations: flood occurs, you have to pay $C_{21}=L_{a}+L_{u}$ tokens; flood doesn't occur, you pay nothing, $C_{22}=0$."

For the matter of convenience, in the remainder of the study $P_{y}$ is written as $\left(C_{11}, p, C_{12}, 1-\right.$ $p)$ and $P_{n}$ as $\left(C_{21}, p, C_{22}, 1-p\right)$.

The experiment was carried out two rounds (indicated as "Ex1" and "Ex2") with different values of $p$ and $C$ are then executed, and the corresponding prospect choices made by the participants are recorded. The data collected in Ex 1 are used to build decision models, whereas the data collected in Ex2 are used to test/validate the accuracy of both model simulations. In order to facilitate the quantification analysis, the decision-making problems were designed in such a way that the participants tend to perceive the two prospects as equally desirable, using the concept of certainty equivalent (CE) proposed in Tversky and Kahneman (1992) and this procedure is exemplified as follows: suppose $p=10 \%$ in this decision-making problem. To get the $C E$ of $P_{y}$, the consequence values $C_{11}$ and $C_{12}$ related to the decision "issue a warning" $\left(D_{y}\right)$ are fixed, i.e., fixing $P_{y}$ as $(-1700,10 \%,-900,90 \%)$. As mentioned above, $C_{22}$ associated with the decision "do not issue a warning" $\left(D_{n}\right)$ is zero, so only the values of $C_{21}$ can take any value in the range of $\pm 25 \%$ of the expected value of $P_{y}$, i.e., $10 \% \times 1700+$ $90 \% \times 900=980$, which is recommended in KT92. Therefore, for a 10\% probability of flood 
occurrence, the value $\mathrm{C}_{21}$ will make the prospect $P_{n}$ equally desirable than the prospect $P_{y}$ is in the range:

$$
C_{21}=\left(\frac{980 \times(1-25 \%)}{10 \%}, \frac{980 \times(1+25 \%)}{10 \%}\right)=(7350,12250)
$$

Seven equally distanced values of $C_{21}$ within this range were selected for setting the decisionmaking problems, i.e.,7350, 8050, 8750, 9450, 10500, 11200 and 12250. The same exercise is executed to obtain the range of values for $C_{11}$ and $C_{12}$ by fixing the prospect $P_{n}$, while keeping the $E\left(P_{y}\right)$ within the range of $\pm 25 \%$ of $E\left(P_{n}\right)$ for each flood probability considered. This results in a range $(1507.5,2032.5)$ for $C_{11}$ and $(707.5,1232.5)$ for $C_{12}$ for the probability of a flood of $10 \%$.

Following this, 126 different decision-making problems were then generated for Ex 1 and 36 for Ex 2 and solved by 168 professionals specialising in water management. In order to check consistency in the answers, every problem was solved by at least 5 different participants.

\subsubsection{Two decision models}

During my MSc study, two decision models were built based on decision theory (cumulative prospect theory, CPT) and machine learning technique (decision trees, DT) respectively and more details can be checked in Wang (2017). This section gives a brief introduction of the two models.

- CPT model

CPT model is built based on a widely used cumulative prospect theory proposed by Tversky and Kahneman (1992) and contains value functions and weighted probability functions to display the characteristics of decision-makers. The value function has the following form:

$$
v(x)= \begin{cases}x^{\alpha} & \text { for gains } x>0 \\ -\lambda(-x)^{\beta} & \text { for losses } x<0\end{cases}
$$


where $x$ is a potential consequence of the choice; $\alpha, \beta$ are parameters that display characteristics of decision-makers (i.e., risk seeking, risk neutrality or risk aversion). The values of these parameters are $\alpha<1$ and $\beta<1$, which means that risk attitudes are different if the problem is formulated in terms of gains or losses. These parameters make the $v(x)$ function S-shaped, showing a diminishing sensitivity to the risk (the degree of risk aversion or risk seeking decreases as the potential value of losses or gains increases); $\lambda$ denotes risk aversion to losses, and $\lambda>1$ if the degree of risk attitude is more sensitive to losses than to gains.

The weighted probabilities $w(p)$ of gains (positive) and losses (negative) are:

$$
\begin{aligned}
& w^{+}(p)=\frac{p^{\gamma}}{\left(p^{\gamma}+(1-p)^{\gamma}\right)^{1 / \gamma}} \\
& w^{-}(p)=\frac{p^{\delta}}{\left(p^{\delta}+(1-p)^{\delta}\right)^{1 / \delta}}
\end{aligned}
$$

where $p$ is the probability of either gaining or losing, and $\gamma$ and $\delta$ are parameters that define the shape of the function curves. If decision-makers show an inclination to underweight large probabilities and overweigh lower probabilities, then both $\gamma$ (for gains) and $\delta$ (for losses) should be less than 1, making the function take a shape of an inverted " $\mathrm{S}$ ". The difference between the weighted probability $w(p)$ and the actual probability $p$ can be used to define risk attitude. The empirical case in CPT shows that: 1) people tend to behave risk-averse when the probability of losing is high $(w(p)>p) ; 2)$ if $w(p)=p$, people tend to behave risk-neutral; $3)$ if the probability of losing is medium or low $(w(p)<p)$, people tend to behave risk-seeking. However, opposite risk attitudes are observed when gains are involved. This is called the fourfold pattern of risk attitudes. These parameters in value and weighted probability function are estimated by an iteration algorithm described in Wang (2017).

Another important concept is the Certainty Equivalence $(C E)$, defined as the utility $U$ that a decision-maker would have to receive to be different between that $U$ and the expected utility $E(U)$ of an uncertain prospect. $C E$ is usually not equal to the utility value obtained using expected utility theory (e.g., summations of products between probabilities and consequences) and $C E$ affects the probabilities by weights that can be obtained experimentally and shown in Eq. (8.4) which is the final step of the CPT model. 


$$
C E=\sum w(p) \times v(x)
$$

$C E$ simulated by the CPT model is further used to compare among different consequences to draw the decision (issue a warning or not) which is the output of the model.

The CPT is chosen to help understand and interpret decision-making problems in the context of flood forecasting and early warning, which highlights the relevance of changing risk attitudes and the need of analysing the difference between the gamble decision and the decision under uncertain flood situations.

- DT model

Decision Tree (DT) is one of the most used applications in machine learning to classify discrete-valued functions (Mitchell, 1997). A DT consists of a collection of nodes arranged by levels and connected by branches; for a given input that requires to be classified (or instance), a conditional statement is evaluated at the node in the first level; then, depending on the evaluation outcome, a particular path (branch) to another node in the next level is taken, where a new conditional situation is evaluated. This process is repeated until the last level of nodes is reached, which are the final targets or outputs. Once a new set of instances enters the tree, the conditionals are applied and the predicted output for such set is found.

The conditionals, as well as the structure of the nodes and branches, are built using datasets of inputs with known outputs (targets); for this, a number of algorithms have been proposed, including ID3, C4.5 and SLIQ. In this DT model, the ID3 algorithm (Grzymala-Busse, 1993; Quinlan, 1986) is used as a basic, top-down, greedy search approach to constructing the decision trees, due to its capability of handling non-numeric attributes. A detailed explanation can be found in Quinlan (1986). The performance of DT is generally evaluated by CrossValidation (CV), and different methods have been developed to optimise this and other types of classifiers by minimizing the $\mathrm{CV}$ error. This case employed the $k$-fold cross-validation method where the original dataset is randomly divided into $k$ subsets of equal size of which a subset is selected randomly to be the validation set, while the remaining $k-1$ subsets are used as a training set. The training sets are used to build the trees, while the validation set is used to 
test the tree. The differences between the results of the two sets are used to estimate the error. This process is repeated $k$ times with each subset being the validation set and the remaining $k$ 1 subsets being the training sets. The optimal size of the tree is that with the minimal CV error.

The DT model built for this case has four attributes (probability of flood occurrence, potential damage of flood, the cost of issuing a warning, the ratio of damage over cost) and the target is the decision on whether issue a warning or not.

\subsection{Reconstruction and improvement of two decision models}

\subsubsection{Improving the Cumulative Prospect Theory (CPT) model}

As the iteration algorithm applied in the CPT model (see Section 8.1.2) is less effective to estimate the parameters, the new CPT model has been upgraded by employing a nonlinear least squares method and the procedure to re-build the new CPT model based on Tversky and Kahneman (1992) (short for "KT92") is shown in Figure 8.1. 


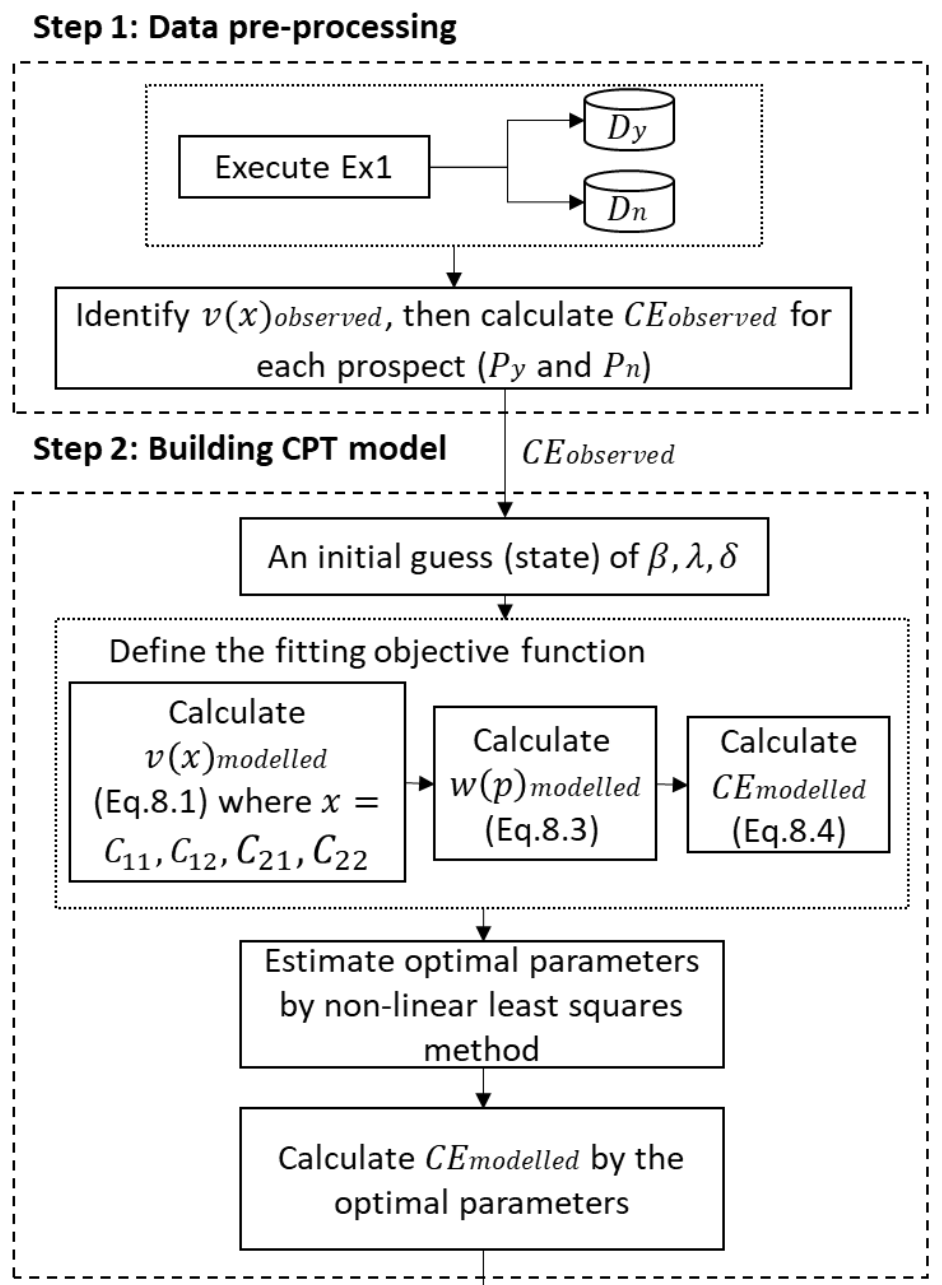

\section{Step 3: Output of CPT model}

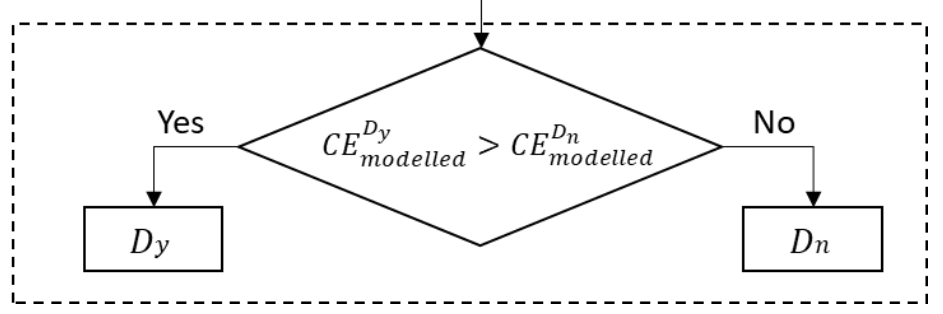

Figure 8.1 Procedure to build and apply the new CPT model based on Cumulative Prospect Theory (CPT).

\section{Step 1. Data pre-processing}

The inputs of step 1 are collected from Ex1, which include the information about each prospect $P_{y}$ and $P_{n}$ (i.e., $p$ and $x=C_{11}, C_{12}, C_{21}, C_{22}$ ), as well as the decision-makers' decisions $D_{y}$ or 
$D_{n}$. Then following KT92, the observed certainty equivalent is calculated to train the new CPT model shown in step 2 . The calculation is further described below:

To obtain the observed certainty equivalent $C E$ of $P_{y}$, participants were observed to change the decision from $D_{n}$ to $D_{y}$ when $C_{21}$ changed from $v 1$ to $v 2$, then the value of $C_{21}$ for calculating $C E$ can be estimated as the average of $v 1$ and $v 2$, which is $v\left(C_{21}\right)_{\text {observed }}^{D_{y}}=(v 1+v 2) / 2$. As $C_{22}$ is zero, the observed certainty equivalent of $P_{y}$ can be calculated as the expected value of $P_{n}$ when participants change decisions, which is $C E_{\text {observed }}^{D_{y}}=-v\left(C_{21}\right)_{\text {observed }}^{D_{y}} \times p+$ $v\left(C_{22}\right)_{\text {observed }}^{D_{y}} \times(1-p)$.

The following decision problem, which was used in the experiment, is now used to show how to calculate the matrix $C$ : the probability of flood occurrence is expected to be $10 \%$. Decisionmakers who issue a warning $\left(D_{y}\right)$ are asked to pay $C_{11}=1700$ if a flood occurs or $C_{12}=900$ if a flood does not occur; and those who do not issue a warning $\left(D_{n}\right)$ would pay $C_{21}=10500$ if it occurs or nothing $\left(C_{22}=0\right)$ if otherwise.

Firstly, in order to find $v(x)_{\text {observed }}^{D_{y}}$, seven values of $C_{21}$, in the range $(7350,12250)$, are used to construct decision-making problems, which are solved by different participants. It is observed that the majority of participants changed their choice from $D_{n}$ to $D_{y}$ when $C_{21}$ went up from 7350 to 8050 . The average between the lowest accepted value (8050) for choosing $D_{y}$ and the highest rejected value (7350) for not choosing $D_{y}$ is then 7700 tokens. Thus, $v\left(C_{21}\right)_{\text {observed }}^{D_{y}}=7700$, and $v\left(C_{22}\right)_{\text {observed }}^{D_{y}}=0$.

Secondly, once $v(x)_{\text {observed }}^{D_{y}}$ is known, the observed Certainty Equivalent of $D_{y}$ can be calculated using Eq. (8.4).

$$
\begin{aligned}
C E_{\text {observed }}^{D_{y}}=\sum v(x) \times p & =-v\left(C_{21}\right)_{\text {observed }}^{D_{y}} \times p+v\left(C_{22}\right)_{\text {observed }}^{D_{y}} \times(1-p) \\
& =-7700 \times 0.1+0 \times(1-0.1)=-770
\end{aligned}
$$


The negative value refers to the problem formulated as losses. The fact that $C E_{\text {observed }}^{D_{y}}$ is lower than the corresponding expected value, i.e., $E\left(D_{y}\right)=-1700 \times 0.1+(-900) \times(1-0.1)=$ -980 , is an indication of a deviation of decision-maker from the rational choice. According to KT92, this difference can reveal the decision-makers' attitude towards risk.

Similarly, $C E_{\text {observed }}^{D_{n}}$ is calculated, but by considering $C_{21}$ and $C_{22}$ fixed to 10500 and 0 respectively, and considering the range $(1507.5,2032.5)$ for $C_{11}$ and the range $(707.5,1232.5)$ for $C_{12}$.

\section{Step 2. Building CPT model}

The objective of this step is to calculate the modelled values of $C E$ by estimating the three parameters $\lambda, \beta$ and $\delta$. The step employs the nonlinear least squares method incorporated with the Levenberg-Marquardt (LM) algorithm by minimizing the sum of the squares of the errors (SSE) between the modelled $C E$ s and observed $C E$ s expressed in Eq. (8.5). It starts with an initial guess of parameters that are the same as the original parameters reported in Tversky and Kahneman (1992). Then LM iteration is applied to get the optimal solutions shown in step 2 of Figure 8.1. Both $C E_{\text {modelled }}$ for the two prospects $P_{y}$ and $P_{n}$ are calculated individually and respectively.

$$
S S E=\sum_{i=1}^{N}\left(C E_{\text {observed }}-C E_{\text {modelled }}\right)^{2}
$$

where $N$ is the number of experiment observations.

\section{Step 3. Output of the CPT model}

The objective of this step is to apply the model to obtain the modelled decision outcome (either modelled $D_{y}$ or $D_{n}$ ) of a given decision problem in the context of flood early warning, formulated as prospects $P_{y}$ and $P_{n}$. The modelled decision-making outcome is triggered by comparing $C E$ of both prospects, depicted at the bottom of Figure 8.1. The modelled decision outcome is obtained as follows: if $C E_{\text {modelled }}^{D_{y}}>C E_{\text {modelled }}^{D_{n}}$, then the decision outcome is $D_{y}$ (issue alarm); otherwise, it is $D_{n}$ (do not issue alarm). 


\subsubsection{Reconstructing the Decision Tree (DT) model}

As the attributes used in the previous DT model (see Section 8.1.2) are four which causes complications in illustrating the results, in the new model, I reduced the number of attributes to two, i.e., the probability of flood occurrence $p$ and cost/loss ratio $r$.

Different from the damage/cost ratio which is one of the attributes used in the previous DT model, the cost/loss ratio applied in the new DT model is calculated based on the reference given by Murphy (1977) in a more accurate way by considering all the combination of consequences:

$$
r=1-\frac{\left(L_{a}+L_{u}\right)-\left(C_{f}+L_{u}\right)}{\left(L_{a}+L_{u}\right)+\left(C_{f}+f\right)-\left(C_{f}+L_{u}\right)}=1-\frac{L_{a}-C_{f}}{L_{a}+f}=\frac{C_{f}+f}{L_{a}+f}
$$

The same experimental data are used to build and train the DT model and the ID3 algorithm is adopted, with data of prospects $P_{y}$ and $P_{n}$ as attributes, and the observed decisions. A sample of instances, specifying attributes and targets, is shown in Table 8.1 where only $p$ and $r$ are the attributes in the new DT model.

Table 8.1 Sample of instances for DT model building.

\begin{tabular}{|c|c|c|c|c|c|c|c|}
\hline \multirow{3}{*}{ Instance } & \multicolumn{6}{|c|}{ Attributes } & \multirow{3}{*}{$\begin{array}{c}\text { Target } \\
\text { D }\end{array}$} \\
\hline & \multicolumn{5}{|c|}{$C$} & \multirow[b]{2}{*}{$p$} & \\
\hline & $\begin{array}{c}C_{11}=C_{f}+ \\
L_{u}\end{array}$ & $C_{12}=C_{f}+f$ & $\begin{array}{c}C_{21}=L_{a}+ \\
L_{u}\end{array}$ & $C_{22}=0$ & $\begin{array}{c}\text { Cost/loss } \\
\text { Ratio } r\end{array}$ & & \\
\hline 1 & 1700 & 900 & 7350 & 0 & 0.14 & 0.1 & $\mathrm{D}_{\mathrm{n}}$ \\
\hline 2 & 1700 & 900 & 8050 & 0 & 0.12 & 0.1 & $\mathrm{D}_{\mathrm{y}}$ \\
\hline 3 & 1700 & 900 & 9450 & 0 & 0.10 & 0.1 & $\mathrm{D}_{\mathrm{y}}$ \\
\hline$\ldots$ & $\ldots$ & $\ldots$ & $\ldots$ & $\ldots$ & $\ldots$ & $\ldots$ & \\
\hline
\end{tabular}

\subsubsection{Evaluation of model performance}

The last step is to evaluate the new CPT and DT models performance by using the same approach in Wang (2017) which is followed: the accuracy of simulation results is calculated by using the validation sets collected in Ex2. 


$$
\text { Accuracy }=(M / N) \times 100 \%
$$

where $N$ is the total number of observations (decisions) obtained in Ex2 and $M$ is the number of the modelled decisions which coincide with the corresponding observed decisions. The higher accuracy indicates a better performance.

\subsection{Results}

\subsubsection{The new CPT model generation with data collected in Ex1}

Table 8.2 presents the comparison between the expected value, which can be regarded as the corresponding "rational choice", and the certainty equivalent $C E_{\text {observed }}$ and $C E_{\text {modelled }}$ of both prospects at different probabilities, based on the observed decisions in Ex1. It can be noted how these values deviate from each other, which provides a mean to explain the underlying reason why certain decisions were made. For example, for a flood event estimated to occur with a probability of $1 \%$, the actual losses for $D_{n}$ is 105 tokens and for $D_{y}$ is 908 tokens. The rational decision is clearly "do not issue a warning" because of its small expected loss. However, in the mind of the majority of participants this difference is not so pronounced, as $D_{n}$ brings a loss of 159 (slightly higher than 105), whereas $D_{y}$ brings a loss of 250 (way lower than 908), although still triggering the "do not to issue a warning" decision. Participants slightly overestimated the consequences of selecting $D_{n}$ and largely underestimate the consequences of selecting $D_{y}$. Comparing the observed and modelled $C E$, it is found that generally the new CPT model can capture the observed $C E$ expect when estimating the $C E$ of $P_{y}$ at extreme low and high probabilities ( $p=1 \%, 5 \%, 95 \%, 99 \%)$. It can be explained as people perceive a lower cost for issuing alarms in unlikely flood events but a higher cost in high probability events. Although such large deviation will not affect the final decision driven by the comparison between $C E$ of $P_{y}$ and $C E$ of $P_{n}$, it may inspire a re-consideration on improving the CPT for simulating extreme decisions. 
Table 8.2 Expected values of each prospect compared with their observed certainty equivalents.

\begin{tabular}{|c|c|c|c|c|c|c|}
\hline & \multicolumn{2}{|c|}{ Do not issue a warning $D_{n}(-10500,0)$} & \multicolumn{3}{c|}{ Issue a warning $D_{y}(-1700,-900)$} \\
\hline$p$ & $\mathrm{E}\left(D_{n}\right)$ & $C E_{\text {observed }}$ & $C E_{\text {modelled }}$ & $\mathrm{E}\left(D_{y}\right)$ & $C E_{\text {observed }}$ & $C E_{\text {modelled }}$ \\
\hline $1 \%$ & -105 & -159 & -104 & -908 & -255 & -949 \\
\hline $5 \%$ & -525 & -595 & -528 & -940 & -595 & -981 \\
\hline $10 \%$ & -1050 & -1130 & -1065 & -980 & -770 & -1012 \\
\hline $25 \%$ & -2625 & -2425 & -2693 & -1100 & -1012.5 & -1086 \\
\hline $50 \%$ & -5250 & -5200 & -5430 & -1300 & -1110 & -1200 \\
\hline $75 \%$ & -7875 & -8175 & -8167 & -1500 & -1387.5 & -1337 \\
\hline $90 \%$ & -9450 & -10820 & -9796 & -1620 & -1480 & -1460 \\
\hline $95 \%$ & -9975 & -11210 & -10332 & -1660 & -1995 & -1522 \\
\hline $99 \%$ & -10325 & -11392 & -10757 & -1692 & -2079 & -1597 \\
\hline
\end{tabular}

Note: two outcomes of each prospect are given in two columns with expected values at left and observed certainty equivalents at right; the probability is given in the first column. For example, the value of -159 in the second column upper right corner is the certainty equivalent of the prospect $(-10500, .01 ; 0, .99)$.

The risk attitudes of the majority of participants can be analysed by comparing the flood forecast probabilities $p$ and the weighted (perceived) probabilities $w(p)$ which is calculated by Eq. (8.3) with $\delta=1.01$ for $D_{n}$ and $\delta=0.73$ for $D_{y}$ estimated by the nonlinear least square method:

$$
\begin{aligned}
w^{-}(p)_{\text {modelled }}^{D_{n}} & =\frac{p^{1.01}}{\left(p^{1.01}+(1-p)^{1.01}\right)^{1 / 1.01}} \\
w^{-}(p)_{\text {modelled }}^{D_{y}} & =\frac{p^{0.73}}{\left(p^{0.73}+(1-p)^{0.73}\right)^{1 / 0.73}}
\end{aligned}
$$

From Figure 8.2, several findings on the risk attitudes can be reported:

First, the observed weighting function of the prospect $P_{n}(-10500, p, 0,1-p)$ linearly coincides with the reference line, exhibiting a risk neutral attitude.

Second, for prospect $P_{y}(-1700, p,-900,1-p)$, a tendency for risk aversion is observed when $p<25 \%$. Then, a clear risk attitude shift is observed in the range $25 \%<p<50 \%$, from risk aversion to risk seeking. In addition, for $p>50 \%$, people tend to over-weigh the losses caused by the potential flood damage but under-weigh the cost of issuing a warning (even false alarming included). 
Third, for $p<25 \%$, people show risk aversion to both decisions, but the attitude of more risk aversion leads to the choice of not to issue a warning because of more overestimation on the cost of issuing a warning.

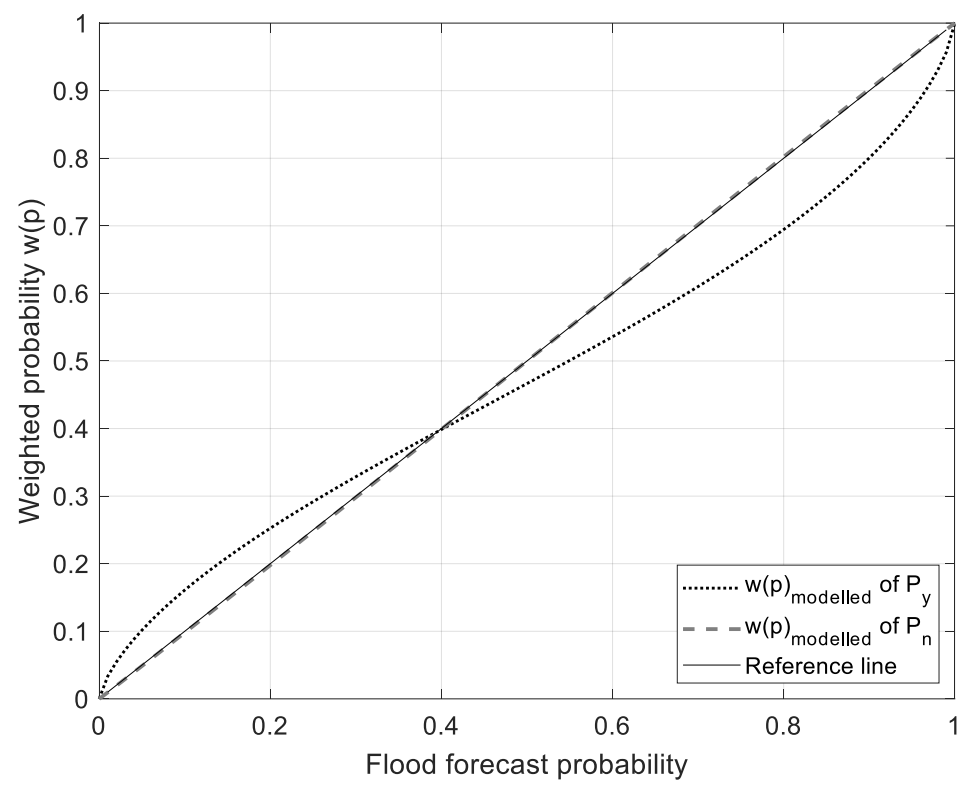

Figure 8.2 Flood forecast probability versus the modelled and observed weighted probabilities for prospects $\mathrm{P}_{\mathrm{y}}$ and $P_{n}$.

Table 8.3 presents the estimated values of the parameters $\delta, \beta$ and $\lambda$ of the two prospects comparing with the parameters recommended in KT92. It can be seen that the value of $\delta$ for $P_{n}$ is slightly higher than 1.0, which breaks KT92's rule of diminished sensitivity with the increase of probability ( $\delta$ should less than 1.0). This means that although generally, people are risk neutral but tend to be more risk-averse with the increase of flood forecast probability. Besides, the higher the probability of flood occurrence, the larger the flood damage perception (see Table 8.2). However, regarding $P_{y}$, people show a diminishing risk sensitivity $(\delta<1)$ as the probability of flood occurrence increases. It should be noted that although the CPT model cannot perfectly capture $w(p)_{\text {observed }}$ of $P_{n}$, it correctly reflects the relationship between $D_{n}$ and $D_{y}$, i.e., the relative location of observations in Figure 8.2 at different probabilities. This gives important insights for applying the model in future research. It also shows the limitation of the new CPT model when estimating risk attitudes because the original parameters defining the 
shape of function curves obtained by KT92 are based on analysing the numerous simpler monetary gamble problems, not involving a serious societal concern which may potentially affect the decision-makers' attitudes to risk.

Table 8.3 Parameters of new CPT models for losses (where KT92 refers to the original parameters reported in Tversky and Kahneman (1992)).

\begin{tabular}{|c|c|c|c|}
\hline Parameter & $\delta$ & $\beta$ & $\lambda$ \\
\hline KT92 & 0.69 & 0.88 & 2.25 \\
\hline $\begin{array}{c}\text { Do not issue a warning } \\
\left(D_{n}\right)\end{array}$ & 1.01 & 0.91 & 2.38 \\
\hline Issue a warning $\left(D_{y}\right)$ & 0.73 & 0.88 & 2.35 \\
\hline
\end{tabular}

\subsubsection{The new DT model generation with data collected in Ex1}

The DT model is generated using the data of Ex1 arranged as shown in Table 8.1. Several trees were generated, optimised, applied and analysed, which are presented here due to the space limitations. However, an optimal tree of particular interest is shown (see Figure 8.3), which allows for analysing the relation between the potential losses and probabilities of a flood. This tree was built using two attributes, namely the probability of flood occurrence $p$, and the cost/loss ratio estimated with Eq. (8.7).

The results of the new DT model reveal three main findings: 1) when the probability of flood is less than $7.5 \%$, almost all people decide not to issue a warning $\left.\left(D_{n}\right) ; 2\right)$ when the probability of flood is between $7.5 \%$ and $17.5 \%$, the majority of people changed their decision and took "issue a warning" $\left.\left(D_{y}\right) ; 3\right)$ for flood probabilities higher than $17.5 \%$, the decision seems to depend on the value of $r$ : if $r<0.81$, people tend to decide to issue a warning $\left(D_{y}\right)$, and decide not to do it $\left(D_{n}\right)$ otherwise. 


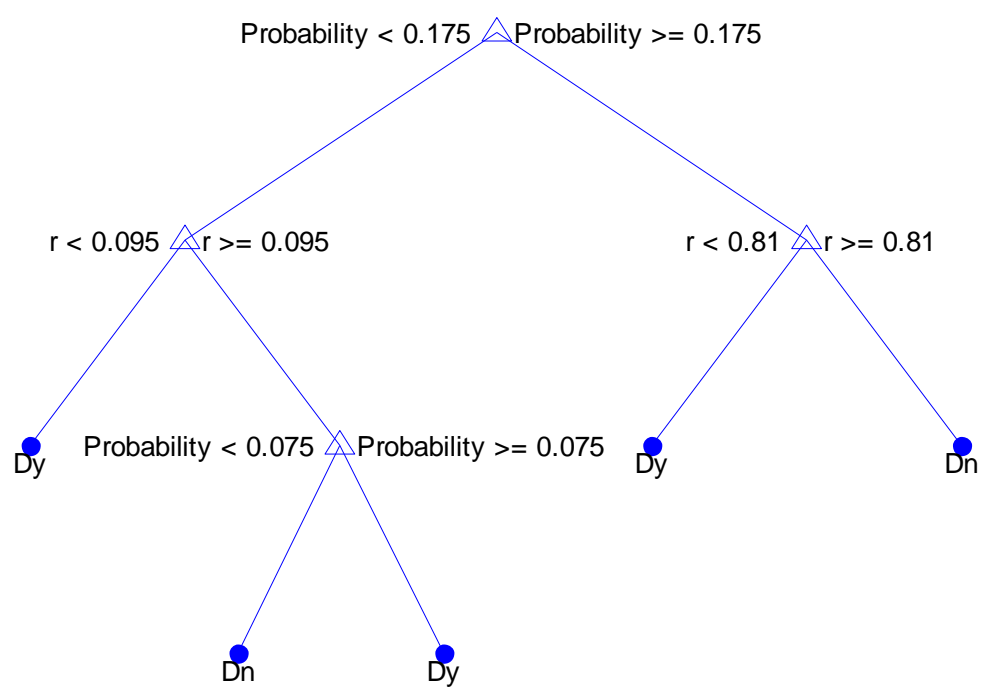

Figure 8.3 Optimal decision tree, where Probability refers to the probability of flood occurrence $\mathrm{p}$, and $\mathrm{r}$ refers to the cost/loss ratio.

The new DT model exhibits a more complex relationship between the cost/loss ratio and the probability of flood occurrence than the cost/loss ratio models that Thompson (1952) and Murphy (1976) suggested. A heavy preference for $D_{y}$ when $r<0.81$ and, simultaneously, when the $p>17.5 \%$ is observed. These values are boundaries in which decisions change. The existence of these limits could be explained from the risk attitudes, which generally change with the change of probability; also, people making decisions may not base them on monetary values only, but rather consider other aspects such as the disadvantages of generating false alarms, like the effect of disturbing the daily activities of residents living in the flood-prone area.

\subsubsection{Performance of the new CPT and DT models tested by data collected in Ex2}

Ex 2 was executed to test the two models. The following numeric example is given to show how the new CPT model is applied to simulate the decision made by a participant:

The decision-making problem is presented as follows: "Flood is expected with 35\% probability. If you issue a warning you must pay 3400 tokens if a flood occurs, or 2500 tokens 
if it doesn't occur; if you don't issue a warning you must pay 10500 tokens if a flood occurs, otherwise pay nothing if it doesn't occur".

The first step is to calculate the weighted probability of each choice. Using $p=35 \%$ in both weighting functions of $D_{y}(\delta=0.73)$ and $D_{n}(\delta=1.01)$, it yields:

$$
\begin{aligned}
& \text { for } D_{y}: \quad w 1^{-}(35 \%)=\frac{35 \% 0.73}{\left(35 \% 0^{0.73}+(1-35 \%)^{0.73}\right)^{1 / 0.73}}=0.364 \\
& w 1^{-}(65 \%)=\frac{65 \% \%^{0.73}}{\left(65 \% \%^{0.73}+(1-65 \%)^{0.73}\right)^{1 / 0.73}}=0.636 \\
& \text { for } D_{n}: \quad w 2^{-}(35 \%)=\frac{35 \%{ }^{1.01}}{\left(35 \% \%^{1.01}+(1-35 \%)^{1.01}\right)^{1 / 1.01}}=0.349 \\
& w 2^{-}(65 \%)=\frac{65 \% \%^{1.01}}{\left(65 \%^{1.01}+(1-65 \%)^{1.01}\right)^{1 / 1.01}}=0.651
\end{aligned}
$$

The second step is to obtain the value of each term for both choices using the value functions (Eq. (8.1)). For $D_{y}$ :

$$
\begin{aligned}
& v\left(C_{11}\right)=-\lambda(-x)^{\beta}=-2.35 \times 3400^{0.88}=-3011.4, \text { if a flood occurs. } \\
& v\left(C_{12}\right)=-\lambda(-x)^{\beta}=-2.35 \times 2500^{0.88}=-2297.5, \text { if a flood does not occur. }
\end{aligned}
$$

And for $D_{n}$ :

$$
\begin{aligned}
& v\left(C_{21}\right)=-\lambda(-x)^{\beta}=-2.38 \times 10500^{0.91}=-10860.7, \text { if a flood occurs. } \\
& v\left(C_{22}\right)=0 \text { if a flood does not occur. }
\end{aligned}
$$

Finally, the Certainty Equivalent of each choice is calculated using Eq. (8.4).

$$
\begin{gathered}
C E\left(D_{y}\right)=\sum(w \times v)=0.364 \times-3011.4+0.636 \times-2297.5=-2557.4 \\
C E\left(D_{n}\right)=\sum(w \times v)=0.364 \times-10860.7+0.651 \times 0=-3953.3
\end{gathered}
$$

As $C E\left(D_{y}\right)>C E\left(D_{n}\right)$, issuing a warning $\left(D_{y}\right)$ is the CPT modelled decision.

All decision-making problems are simulated by the new CPT model using the method described above. Simulations and observations (the participants' decisions in Ex2) are compared using Eq. (8.7) to evaluate the model performance. Figure 8.4 categorised the 
decision-making problems of Ex2 into 45 groups according to their forecast probabilities and consequences which are translated into cost/loss ratio $r$. The figure presents the number of choosing $D_{y}$ of each category and the percentage is calculated by the number of choosing $D_{y}$ divided by the total number of decisions, indicated by a colour scale, comparing the observed results with modelled results by the new CPT and DT models respectively. Results show that the accuracy of the new CPT model is $76 \%$, while the new DT model is $81 \%$. Therefore, a preliminary conclusion is that the DT model performs better in simulating individual decisions than the new CPT model, for this particular case. In addition, the benchmark cost/loss model gives an accuracy of $72 \%$, which is lower than both new CPT and DT models developed in this study.

\begin{tabular}{|c|c|c|c|c|c|c|c|c|c|c|c|c|c|c|c|c|c|}
\hline & \multicolumn{5}{|c|}{ Observed $D_{y}$ decisions } & \multicolumn{6}{|c|}{ Modelled $D_{y}$ by CPT } & \multicolumn{6}{|c|}{ Modelled $D_{y}$ by DT } \\
\hline$[0,0.1)$ & 2 & 7 & 7 & 7 & 7 & {$[0,0.1)$} & 0 & 3 & 7 & 7 & 7 & {$[0,0.1)$} & 7 & 7 & 7 & 7 & 7 \\
\hline$[0.1,0.2)$ & 2 & 2 & 4 & 4 & 4 & {$[0.1,0.2)$} & 0 & 0 & 4 & 4 & 4 & {$[0.1,0.2)$} & 0 & 0 & 4 & 4 & 4 \\
\hline$\Xi[0.2,0.3)$ & 3 & 1 & 4 & 5 & 5 & {$[0.2,0.3)$} & 0 & 0 & 5 & 5 & 5 & {$[0.2,0.3)$} & 0 & 0 & 5 & 5 & 5 \\
\hline$\stackrel{\circ}{\frac{O}{\sigma}}[0.3,0.4)$ & 1 & 0 & 1 & 1 & 1 & {$[0.3,0.4)$} & 0 & 0 & 1 & 1 & 1 & {$[0.3,0.4)$} & 0 & 0 & 1 & 1 & 1 \\
\hline $\mathscr{\infty}[0.4,0.5)$ & 1 & 0 & 2 & 2 & 2 & {$[0.4,0.5)$} & 0 & 0 & 0 & 2 & 2 & {$[0.4,0.5)$} & 0 & 0 & 2 & 2 & 2 \\
\hline 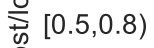 & 2 & 2 & 4 & 4 & 4 & {$[0.5,0.8)$} & 0 & 0 & 0 & 4 & 4 & {$[0.5,0.8)$} & 0 & 0 & 4 & 4 & 4 \\
\hline $\mathcal{U}[0.8,1.0)$ & 1 & 1 & 1 & 1 & 2. & {$[0.8,1.0)$} & 0 & 0 & 0 & 2 & 3 & {$[0.8,1.0)$} & 0 & 0 & 1 & 1 & 3 \\
\hline$[1.0,1.5)$ & 2 & 0 & 1 & 2 & 4 & {$[1.0,1.5)$} & 0 & 0 & 0 & 0 & 3 & {$[1.0,1.5)$} & 0 & 0 & 0 & 0 & 4 \\
\hline$>=1.5$ & 0 & 0 & 0 & 3 & 3 & $>=1.5$ & 0 & 0 & 0 & 0 & 0 & $>=1.5$ & 0 & 0 & 0 & 0 & 6 \\
\hline & $\begin{array}{l}0.01 \\
\text { Floc } \\
\text { Perce }\end{array}$ & & & & & & $\begin{array}{l}0.01 \\
\text { Flo }\end{array}$ & & & & & & $\begin{array}{c}0.0 \\
\text { Flc }\end{array}$ & 10 & & & \\
\hline & 0 & & 10 & & & 30 & 40 & 5 & & 60 & & 70 & 30 & 90 & & 100 & \\
\hline
\end{tabular}

Figure 8.4 The number of majority decisions observed and simulated by the new CPT and DT models.

\subsection{Application of new CPT and DT models}

The proposed new models can be applied to predict majority decisions in various decisionmaking problems in the context of early warning flood forecasting for problems formulated in terms of prospects $P_{y}$ and $P_{n}$. In order to show its applicability, I used the case presented by Ramos et al. (2013), who analysed how the addition of probabilistic flood forecast information can influence decision making on flood control measures. The game consisted of managing a 
gate, which is the inlet of a retention basin designed to protect a fictitious town. The decision they had to make was whether to open the gate or not. The game ${ }^{9}$ was played in six rounds with different forecasted probabilities of flood and played by 145 participants. A description of the consequences of each decision is presented below:

- If the participant opens the gate, the retention basin is flooded and the affected farmers demand compensation for flooding their land: the cost of opening the gate is 2000 tokens;

- If the participant decides not to open the gate and a flood occurs, the town is flooded and they have to pay a fine of 7000 tokens.

This decision game can be formulated in terms of prospects $P_{y}$ and $P_{n}$, as follows : $P_{y}:(-2000, p, 0,1-p)$, and $P_{n}:(-7000, p, 0,1-p)$, with the following considerations: 1) the six probabilities of flood occurrence; 2) the cost of opening the gate is considered related to that of issuing a warning; 3) the penalty for not opening the gate when a flood occurs is considered related to the flood damage; 4) the cost/loss ratio can be regarded as the cost of opening the gate (2000 tokens) over the penalty for not opening the gate ( 7000 tokens) it and equals approximately to $r=0.286$. Therefore, the decision simulated by the cost/loss model is to open the gate if $p>0.286$ and not to open it otherwise.

Although all individual choices were recorded, the analysis is made to observe only the majority of individual choices, as in the previous exercise. The CPT and DT model outputs, as well as the decisions computed following the cost/loss model, are summarized in Table 8.4.

Table 8.4 Decisions taken by participants and simulated results by three models.

\begin{tabular}{|c|c|c|c|c|c|c|c|}
\hline $\begin{array}{l}\text { Decision } \\
\text { Making }\end{array}$ & \multirow{2}{*}{$\begin{array}{c}\text { Probability } \\
\text { of Flood }\end{array}$} & \multicolumn{3}{|c|}{ Answers collected (145 participants) } & \multicolumn{3}{|c|}{ Simulated decision } \\
\hline Rounds & & Open gate & $\begin{array}{c}\text { Do not open } \\
\text { gate }\end{array}$ & $\begin{array}{l}\text { Majority } \\
\text { decision }\end{array}$ & $\begin{array}{l}\text { The new } \\
\text { CPT Model }\end{array}$ & $\begin{array}{c}\text { The new } \\
\text { DT Model }\end{array}$ & $\begin{array}{c}\text { Cost/loss } \\
\text { Model }\end{array}$ \\
\hline
\end{tabular}

\footnotetext{
${ }^{9}$ This game was played, and data were collected by Assco. Prof. Dr Leonardo Alfonso* in the past six years.

* Email: 1.alfonso@un-ihe.org

* Department of Hydroinformatics and Socio-Technical Innovation, IHE Delft Institute for Water Education.
} 


\begin{tabular}{|l|c|c|c|c|c|c|c|}
\hline Round 1 & $9.74 \%$ & 40 & 105 & No & No & Yes & No \\
\hline Round 2 & $48.27 \%$ & 125 & 20 & Yes & Yes & Yes & Yes \\
\hline Round 3 & $12.53 \%$ & 25 & 120 & No & No & Yes & Yes \\
\hline Round 4 & $23.59 \%$ & 57 & 87 & No & No & No & No \\
\hline Round 5 & $20.10 \%$ & 30 & 115 & No & No & No & No \\
\hline Round 6 & $53.17 \%$ & 141 & 4 & Yes & Yes & Yes & Yes \\
\hline
\end{tabular}

As Table 8.4 shows, all three models generally perform well in simulating the decision of the majorities. It can be seen that people's decisions do not always follow the cost/loss model. However, the new CPT model successfully predicted participants' decisions in the six rounds, while the new DT model failed two times (rounds 1 and 3).

Although Ramos' game has a different background and description of flood risk from the experiments used to build the models, the new CPT model shows stronger applicability to predict decisions, which can be attributed to the fact that models based on decision theory/rules can simulate the average characteristics of individual decision-making. In contrast, the applicability of the data-driven DT model usually differs from case to case, depending on the decision-making context. It is worth noting that the new DT model trained using the data of the experiments only shows the majority decisions at different probability and consequences and it is less effective in predicting decision-making in new situations. It is also noted that yet more experiments are needed to establish a definitive conclusion.

\subsection{Summary and remarks}

The study carried out in this chapter is based on a prior analysis of decision making under uncertainty done in Wang (2017), however, it advances further to reconstruct two decision models: CPT (cumulative prospect theory) and DT (decision tree) models using the dataset collected from a survey-based experiment where several sets of one-time binary decisionmaking problems were formulated. The new CPT model is shown to have greatly improved in estimating the parameters while the new DT model works more effectively by refining the attributes. The two new models, though totally different in nature, are further used to analyse different risk attitudes (i.e., risk aversion, risk neutrality and risk seeking) and majority decisions according to different consequences of decisions. The usability of both new models 
was validated by feeding them with another new dataset which was collected in a continuous experiment over 6 years by executing the decision-making experiment presented in Ramos et al. (2013) and compared with the traditional cost/loss model widely applied in flood forecast early warning (FFEW) system.

The following conclusions can be drawn:

1) Risk attitude is a main underlying factor to drive decisions and it varies mainly depending on the probability of flood events. People's attitude to potential flood damage is found to be risk-neutral (with a slight risk-averse) when the flood occurrence probability is low (less than $5 \%$; an increase in probability reflects a progressive increase of risk aversion. However, people's attitude to taking precautionary actions change from risk aversion to risk seeking with an increase of flood probability. The majority of individuals tend to change their minds when the probability of a flood is between $25 \%$ and $50 \%$. Such different and changed attitudes lead to their decisions: for low probabilities of a flood event, people prefer not to take precautionary action; for high probabilities of a flood event, people choose to take action because they become risk averse to potential damage.

2) Although the cost/loss ratio was not used by the participants as a method to make a decision, the a-posteriori evaluation of this value appears to be an important driver to decisions. In the context of flood forecasting and early warning, a heavy preference for not taking precautionary actions is found when the ratio is higher than 0.81 and, simultaneously, when the probability of flood occurrence is less than $17.5 \%$.

3) Although both proposed decision-making models perform good on predicting decisions collected for the testing sets ( $76 \%$ accuracy for the CPT model and $81 \%$ for the DT model), the application shows that the CPT model performs better on predicting similar decision problems than the DT. A possible reason is that the CPT model can simulate the average characteristics of people (risk attitudes and their changing triggers) and shows a tendency of choices while the data-driven DT model is specific to the case for which the training data is provided. 
4) Compared with the traditional cost/loss model, the two new developed models can take the information of probability into account and show a better performance in Ramos's game. 



\section{Chapter 9 Conclusion}

The thesis is devoted to proposing a comprehensive framework for quantitative modelling of hydroclimatic extremes in the perspective of climate change and the proposed 7 research questions have been addressed. There are three main parts in the thesis: 1) hydroclimatic extreme quantification which includes a novel toolbox developed for spatial random sampling in grid-based data analysis, spatiotemporal variation of observed hydroclimatic extremes under the assumption of non-stationarity, and the quantification of the nonstationary link between observed and climate projected hydroclimatic extremes, and a further extension to analyse the multivariate hydroclimatic extremes; 2) the pattern recognition on hydroclimatic extremes which includes a novel toolbox for identifying and classifying the pattern of hydroclimatic extremes and further incorporated into AI-based analysis such as a convolution neural network $(\mathrm{CNN})$; 3) decision-making under uncertainty on hydroclimatic extremes in the context of flood forecasting and early warning that is discussed and simulated by both theory-driven and data-driven models to enhance the understanding of the decision-making process thereby the communication between decision-makers and modellers.

\subsection{Innovations and contributions}

The innovations and main contributions are demonstrated following the normal research path that considers sampling and data analysis, modelling, further applications, and decisionmaking, which is presented below:

- Sampling and data analysis 
To process large-sized grid-based hydroclimatic datasets which need to be diagnosed and further used in the study, a novel, open-source SRS-GDA toolbox (short for "spatial random sampling toolbox for grid-based data analysis", presented in Chapter 2) was developed, aiming to address the research question Q1 by providing random spatial sampling of grid-based quantities with various constraints: shape, size, location, dominant orientation and resolution. Four functions are involved and highlighted as:

1) Boundary recognition.

2) Data extraction and resolution conversion.

3) Randomization of samples' location, shape and size.

4) Self-validation for diagnosing samples.

This toolbox is a fundamental tool to support the spatiotemporal quantification on hydroclimatic extremes in this study. And the wide applicability of the toolbox should be highlighted that it can address the very needs of many climate change related studies on spatialtemporal diagnostics of any grid-based hydroclimatic datasets.

\section{- Quantitative modelling}

Both univariate and multivariate quantitative modelling frameworks have been proposed and developed in the thesis.

The univariate quantitative modelling framework is proposed in Chapter 3 and Chapter 4 to address research questions Q2, Q3 and Q4 by providing a new perspective to understanding the heterogeneity of hydroclimatic extreme (i.e., rainfall extreme in the study) distribution over space and time driven by the complex interactions among climate, geographical features, and the practical sampling approaches. The main contributions are:

1) Quantified the spatial variation of area-orientated extremes by developing generalised linear regression models with respect to the sizes, shapes and locations of a large number of study regions.

2) Proposed a nonstationary probability distribution model by assuming a time-varying set of parameters where the index of climate change is significant and incorporated the Markov- 
Chain Monte-Carlo method to estimate the nonstationary parameters and assess model uncertainty.

3) Linked the spatial and temporal patterns to the large-scale climatology and discussed the implication of the modelling parameters to flood risk management.

This quantitative framework has been further applied in two commonly used climate projection (UKCP18 and ERA20CM) datasets in Chapter 5 and the nonstationary link between observed and climate projected extremes has been quantified to answer the research question Q5. Such quantification links offer insight into the utility of climate projection datasets when extreme quantities instead of the averages are at stake in applications, which should be highlighted.

The multivariate quantitative modelling framework has also been developed and further applied in a case study in Chapter 6 with the following innovations and contributions:

1) Nonstationary multivariate frequency analysis for compound floods driven by meteorological and oceanographic drivers.

2) This framework is comprehensive by incorporating many techniques such as the Block Bootstrapping Man-Kendal test, the rolling window method associated with traditional correlation analysis methods, Bayesian Markov-Chain Monte-Carlo method.

\section{- Pattern identification and recognition}

I further linked the SRS-GDA toolbox to the machine learning technique to realise an effective and accurate pattern recognition method for hydroclimatic extreme patterns extraction. In this application, a novel toolbox entitled "the Spatial Pattern Extraction and Recognition" (SPER, presented in Chapter 7) was developed to address the research question Q6 and to facilitate automatic identification and classification of extreme patterns (i.e., rainfall extreme as an example in the study) of any arbitrary hydroclimatic grid-based datasets. Four functions of the toolbox can be highlighted as:

1) Boundary detection of the hydroclimatic extreme patterns.

2) Clustering analysis of the hydroclimatic extreme patterns. 
3) Attribute extraction includes automatic calculating and detecting the spatial features and hydroclimatic features of patterns.

4) Pattern classification by the quantitative attributes.

The main contributions with the support of the toolbox are:

1) To execute the ROI/catchment-based analysis which requires spatial features correlation analysis such as location, size, shape and orientation; and pattern identification where a study of exploring the top 3 dominating daily rainfall patterns in GB is demonstrated in this thesis; and the monitoring of the track of rainfall centre which can be used for forecasts.

2) The toolbox presents great potential in auto-labelling clusters to support deep learning of complex environmental spatial-temporal features over large datasets, demonstrated by an example of convolution neural network $(\mathrm{CNN})$ which is able to pick up the labelled rainfall patterns with high accuracy.

\section{- Decision-making under uncertainty}

As the work presented above is to achieve a better quantification of univariate or multivariate hydroclimatic extremes in the perspective of climate change spatially and temporally and to make more effective use of forecasting data by constructing the link between extremes or training machine to recognise the patterns, the final step is how to make the decisions by using the quantitative information (Chapter 8) and how much it can affect the risk attitude to the public (e.g., the research question Q7). Based on a previous study, two decision models (theorydriven and data-driven) have been rebuilt and improved and tested by data collected via several rounds of laboratory-style experiments, where 168 individuals with the water-related background are confronted with binary decision problems based on probabilistic flood forecasts in different contexts. The main contributions are:

1) Risk attitudes (risk aversion, risk neutral, risk seeking) of decision-makers and how they change with different flood forecasting information were revealed and quantified by a theory-driven model.

2) The majority of decisions were simulated by both theory-driven and data-driven models however the data-driven model outperforms the theory-driven model. 
3) Two models were also tested on an earlier-performed decision-making game by Ramos et al. (2013) executed by 145 participants in the past 6 years. In this case, the theory-driven model outperforms the data-driven model, which confirms the strong risk-averse tendency of decision-makers in the context of flood forecasting and early warning.

\subsection{Future work}

Climate change is one of the hottest research topics since the terms such as global warming and climate change were defined and popularised since the 1980s (Burroughs, 2007). It has already affected and kept affecting every region of the world in multiple ways which are not just about increasing temperature but bringing some potentially irreversible changes to the environment (wetness and dryness), to winds, snow and ice, coastal areas and oceans (Shukla et al., 2019). Nowadays, reducing or halting climate change is regarded as an urgent matter especially when our world is experiencing a more extensive crisis due to the global COVID19 pandemic outbroken in 2019 which has lasted until now. Especially in this year (2021), extreme weather and events which are unprecedented in thousands of years occurred more frequently and globally. For example, just by the time I nearly complete this thesis, from 18 to 27 July, Zhengzhou, Henan Province of China encountered a flash flood during torrential rainfall (see Figure 9.1) with the highest record of $720 \mathrm{~mm}$ which "smashed historical records" (Zhongming et al., 2021). The city of London in the UK was also hit by a flash flood caused by heavy downpours and thunderstorms on 25th July. In the meantime, severe floods were recorded to happen in Germany and Belgium on 17-18 July, which also caused huge casualties and losses. Therefore, how to get an accurate and prompt forecast on these unpredictable extreme events and how to mitigate and combat climate change are still a huge challenge for the future. Future work is given below associated with the contribution of the thesis. 


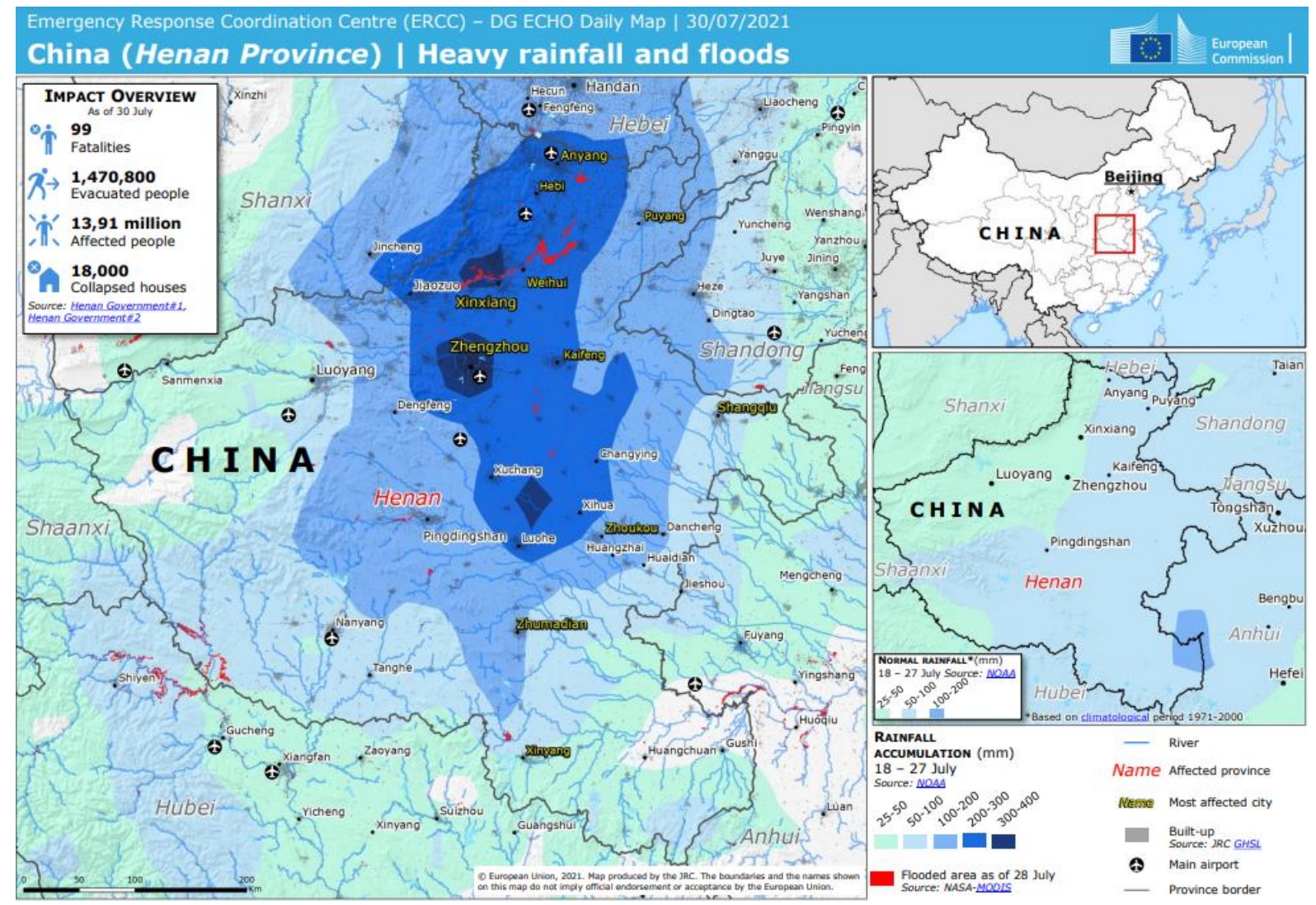

Figure 9.1 Accumulative rainfall (mm) in Zhengzhou from 18 to 27 July 2021 and this figure is produced by ECHO/European Commission (source: https://erccportal.jrc.ec.europa.eu/ECHO-Products/Maps\#/maps/3780

Accessed: 2021-11-02)

This thesis endeavours to contribute to quantifying climate impacts on hydroclimatic extremes by developing a well-tested framework to analyse spatial and temporal changes of hydroclimatic variables such as extreme rainfall, temperature, sea water level and their combination. It also provides a test on climate projections by a nonstationary framework and finds that although they have been improved with rapid development on environmental monitoring techniques, some questions need to be addressed, including 1) they are unable to simulate extremes albeit being good at simulating the average; 2) they may not be able to simulate the time-varying change in climate extreme. Though the bias between observed and climate projected extremes have been diagnosed and quantified in the thesis, further work can be carried out to improve climate models for getting a better simulation by such as correcting the bias, refining the resolution, optimising model structure by using an appropriate level of 
details (a more complex model is not always a better model), involving non-stationarity for tracking/predicting the temporal changes of climate extremes.

This thesis also provides an evidence that artificial intelligence (AI) techniques can indeed help improve and expedite the forecasting and prediction of climate systems by processing enormous amounts of chaos, multi-dimensional data, automatically labelling these large climate modelling data, monitoring and tracking the rainfall cell from the experience where I tried to design a pattern recognition toolbox to be used as a front end that supports AI-based training in tracking and forecasting extremes. How AI techniques can tackle climate change is yet another promising research topic in the future. Further work can be recommended to involve AI technique to climate model for getting a better simulation and forecast on climate extremes.

Last but not least, there is still a challenge on how to convince decision-makers with these complex results produced by climate models and techniques. Although the thesis contributes to understanding decision makers' behaviour and risk attitudes when making decisions relating to risk from flood hazards under uncertainty, understanding flood warning decision-making in practice is still remarkably complex, as it involves a chain of many small decisions, some of which are reversible. A welcoming further study is, therefore, to improve the decision support system by constructing a more user-friendly platform including effective transformation between meteorological forecasts and hydrological response, better data visualisation and explanation for helping decision-makers issue their flood response. 



\section{References}

Aerts, J. C., Botzen, W. J., Clarke, K. C., Cutter, S. L., Hall, J. W., Merz, B., Michel-Kerjan, E., Mysiak, J., Surminski, S., \& Kunreuther, H. (2018). Integrating human behaviour dynamics into flood disaster risk assessment. Nature Climate Change, 8(3), 193-199.

Agarwala, A., Dontcheva, M., Agrawala, M., Drucker, S., Colburn, A., Curless, B., Salesin, D., \& Cohen, M. (2004). Interactive digital photomontage. In ACM SIGGRAPH 2004 Papers (pp. 294-302).

AghaKouchak, A., Chiang, F., Huning, L. S., Love, C. A., Mallakpour, I., Mazdiyasni, O., Moftakhari, H., Papalexiou, S. M., Ragno, E., \& Sadegh, M. (2020). Climate extremes and compound hazards in a warming world. Annual Review of Earth and Planetary Sciences, 48, 519-548.

Akaike, H., Petrov, B. N., \& Csaki, F. (1973). Second international symposium on information theory. In: Akadémiai Kiadó, Budapest.

Alfonso, L., Mukolwe, M., \& Di Baldassarre, G. (2016). Probabilistic flood maps to support decision - making: Mapping the value of information. Water Resources Research, 52(2), 1026-1043.

Alfonso, L., \& Price, R. (2012). Coupling hydrodynamic models and value of information for designing stage monitoring networks. Water Resources Research, 48(8).

Alfonso, L., \& Tefferi, M. (2015). Effects of uncertain control in transport of water in a riverwetland system of the Low Magdalena River, Colombia. In Transport of water versus transport over water (pp. 131-144): Springer.

Alom, M. Z., Taha, T. M., Yakopcic, C., Westberg, S., Sidike, P., Nasrin, M. S., Van Esesn, B. C., Awwal, A. A. S., \& Asari, V. K. (2018). The history began from alexnet: A comprehensive survey on deep learning approaches. arXiv preprint arXiv:1803.01164.

Amaya, D. J., \& Foltz, G. R. (2014). Impacts of canonical and Modoki El Niño on tropical Atlantic SST. Journal of Geophysical Research: Oceans, 119(2), 777-789.

Anderson, T. W., \& Darling, D. A. (1952). Asymptotic theory of certain" goodness of fit" criteria based on stochastic processes. The annals of mathematical statistics, 193-212.

Anderson, T. W., \& Darling, D. A. (1954). A test of goodness of fit. Journal of the American Statistical Association, 49(268), 765-769. 
Anquetin, S., Braud, I., Vannier, O., Viallet, P., Boudevillain, B., Creutin, J.-D., \& Manus, C. (2010). Sensitivity of the hydrological response to the variability of rainfall fields and soils for the Gard 2002 flash-flood event. Journal of Hydrology, 394(1-2), 134-147.

Arabameri, A., Rezaei, K., Cerdà, A., Conoscenti, C., \& Kalantari, Z. (2019). A comparison of statistical methods and multi-criteria decision making to map flood hazard susceptibility in Northern Iran. Science of the Total Environment, 660, 443-458.

Arshad, B., Ogie, R., Barthelemy, J., Pradhan, B., Verstaevel, N., \& Perez, P. (2019). Computer vision and IoT-based sensors in flood monitoring and mapping: A systematic review. Sensors, 19(22), 5012.

Ashok, K., Behera, S. K., Rao, S. A., Weng, H., \& Yamagata, T. (2007). El Niño Modoki and its possible teleconnection. Journal of Geophysical Research: Oceans, 112(C11).

Assani, A., \& Guerfi, N. (2017). Analysis of the Joint Link between Extreme Temperatures, Precipitation and Climate Indices in Winter in the Three Hydroclimate Regions of Southern Quebec. Atmosphere, 8(4), 75.

Atkins, J. P., Burdon, D., \& Allen, J. H. (2007). An application of contingent valuation and decision tree analysis to water quality improvements. Marine Pollution Bulletin, 55(1012), 591-602.

Ausin, M. C., \& Lopes, H. F. (2010). Time-varying joint distribution through copulas. Computational Statistics \& Data Analysis, 54(11), 2383-2399.

Baíllo, A., \& Grané, A. (2009). Local linear regression for functional predictor and scalar response. Journal of Multivariate Analysis, 100(1), 102-111.

Banwell, N., Rutherford, S., Mackey, B., Street, R., \& Chu, C. (2018). Commonalities between disaster and climate change risks for health: A theoretical framework. International Journal of Environmental Research and Public Health, 15(3), 538.

Barrow, H., Tenenbaum, J., Hanson, A., \& Riseman, E. (1978). Computer vision systems.

Barrow, H., Tenenbaum, J., Hanson, A., \& Riseman, E. (1978). Recovering intrinsic scene characteristics. Comput. Vis. Syst, 2(3-26), 2.

Bauer, P., Thorpe, A., \& Brunet, G. (2015). The quiet revolution of numerical weather prediction. Nature, 525(7567), 47-55.

Baumgart, B. G. (1974). Geometric modeling for computer vision: Stanford University.

Beecham, S., Rashid, M., \& Chowdhury, R. K. (2014). Statistical downscaling of multi - site daily rainfall in a South Australian catchment using a Generalized Linear Model. International Journal of Climatology, 34(14), 3654-3670.

Bell, F. (1976). The areal reduction factor in rainfall frequency estimation.

Bergen, J. R., Anandan, P., Hanna, K. J., \& Hingorani, R. (1992). Hierarchical model-based motion estimation. Paper presented at the European conference on computer vision.

Besag, J., \& Diggle, P. J. (1977). Simple Monte Carlo tests for spatial pattern. Journal of the Royal Statistical Society: Series C (Applied Statistics), 26(3), 327-333.

Betta, G., Capriglione, D., Crenna, F., Rossi, G., Gasparetto, M., Zappa, E., Liguori, C., \& Paolillo, A. (2011). Face-based recognition techniques: proposals for the metrological 
characterization of global and feature-based approaches. Measurement Science and Technology, 22(12), 124005.

Bevacqua, E., Maraun, D., Hobæk Haff, I., Widmann, M., \& Vrac, M. (2017). Multivariate statistical modelling of compound events via pair-copula constructions: analysis of floods in Ravenna (Italy). Hydrology and Earth System Sciences, 21(6), 2701-2723.

Bevacqua, E., Vousdoukas, M. I., Zappa, G., Hodges, K., Shepherd, T. G., Maraun, D., Mentaschi, L., \& Feyen, L. (2020). More meteorological events that drive compound coastal flooding are projected under climate change. Communications earth \& environment, 1(1), 1-11.

Beven, K. J., \& Alcock, R. E. (2012). Modelling everything everywhere: a new approach to decision - making for water management under uncertainty. Freshwater Biology, 57, 124-132.

Binder, K., Ceperley, D. M., Hansen, J. P., Kalos, M. H., Landau, D. P., Levesque, D., MuellerKrumbhaar, H., Stauffer, D., \& Weis, J. J. (2012). Monte Carlo methods in statistical physics (Vol. 7): Springer Science \& Business Media.

Bishop, C. M. (2006). Pattern recognition and machine learning: springer.

Bodoque, J. M., Díez-Herrero, A., Amérigo, M., García, J. A., \& Olcina, J. (2019). Enhancing flash flood risk perception and awareness of mitigation actions through risk communication: A pre-post survey design. Journal of Hydrology, 568, 769-779.

Briere, C., Abadie, S., Bretel, P., \& Lang, P. (2007). Assessment of TELEMAC system performances, a hydrodynamic case study of Anglet, France. Coastal engineering, 54(4), 345-356.

Brown, S. J. (2018). The drivers of variability in UK extreme rainfall. International Journal of Climatology, 38, e119-e130.

Bruhn, A., Weickert, J., \& Schnörr, C. (2005). Lucas/Kanade meets Horn/Schunck: Combining local and global optic flow methods. International Journal of Computer Vision, 61(3), 211-231.

Buchecker, M., Salvini, G., Di Baldassarre, G., Semenzin, E., Maidl, E., \& Marcomini, A. (2013). The role of risk perception in making flood risk management more effective. Natural hazards \& earth system sciences, 13(11).

Buontempo, C., Mathison, C., Jones, R., Williams, K., Wang, C., \& McSweeney, C. (2015). An ensemble climate projection for Africa. Climate Dynamics, 44(7-8), 2097-2118.

Burroughs, W. J. (2007). Climate change: a multidisciplinary approach: Cambridge University Press.

Buytaert, W., Celleri, R., Willems, P., De Bievre, B., \& Wyseure, G. (2006). Spatial and temporal rainfall variability in mountainous areas: A case study from the south Ecuadorian Andes. Journal of Hydrology, 329(3-4), 413-421.

Caliński, T., \& Harabasz, J. (1974). A dendrite method for cluster analysis. Communications in Statistics-theory and Methods, 3(1), 1-27.

Cancelliere, A. (2017). Non stationary analysis of extreme events. Water Resources Management, 31(10), 3097-3110. 
Cannon, A. J. (2020). Reductions in daily continental-scale atmospheric circulation biases between generations of global climate models: CMIP5 to CMIP6. Environmental Research Letters, 15(6), 064006.

Cao, A., Esteban, M., Valenzuela, V. P. B., Onuki, M., Takagi, H., Thao, N. D., \& Tsuchiya, N. (2021). Future of Asian Deltaic Megacities under sea level rise and land subsidence: current adaptation pathways for Tokyo, Jakarta, Manila, and Ho Chi Minh City. Current Opinion in Environmental Sustainability, 50, 87-97.

Celleri, R., Willems, P., Buytaert, W., \& Feyen, J. (2007). Space-time rainfall variability in the Paute basin, Ecuadorian Andes. Hydrological Processes: An International Journal, 21(24), 3316-3327.

Chan, S. H., \& Song, Q. (2010). Motivational framework: insights into decision support system use and decision performance. Decision Support Systems. Ceoatia: InTech, 1-24.

Chandler, R. E., \& Wheater, H. S. (2002). Analysis of rainfall variability using generalized linear models: a case study from the west of Ireland. Water Resources Research, 38(10), 10-11-10-11.

Chang, K. T. (2008). Introduction to geographic information systems (Vol. 4). Boston: McGraw-Hill.

Chattopadhyay, A., Hassanzadeh, P., \& Pasha, S. (2020). Predicting clustered weather patterns: A test case for applications of convolutional neural networks to spatio-temporal climate data. Scientific Reports, 10(1), 1-13.

Chebana, F., Ouarda, T. B., \& Duong, T. C. (2013). Testing for multivariate trends in hydrologic frequency analysis. Journal of Hydrology, 486, 519-530.

Chen, C.-Y., \& Klette, R. (1999). Image stitching-Comparisons and new techniques. Paper presented at the International conference on computer analysis of images and patterns.

Chen, S. E., \& Williams, L. (1993). View interpolation for image synthesis. Paper presented at the Proceedings of the 20th annual conference on Computer graphics and interactive techniques.

Chen, Y., Li, Z., Fan, Y., Wang, H., \& Deng, H. (2015). Progress and prospects of climate change impacts on hydrology in the arid region of northwest China. Environmental Research, 139, 11-19.

Ciach, G. J., \& Krajewski, W. F. (2006). Analysis and modeling of spatial correlation structure in small-scale rainfall in Central Oklahoma. Advances in Water Resources, 29(10), 1450-1463.

Clark, M. P., Wilby, R. L., Gutmann, E. D., Vano, J. A., Gangopadhyay, S., Wood, A. W., Fowler, H. J., Prudhomme, C., Arnold, J. R., \& Brekke, L. D. (2016). Characterizing uncertainty of the hydrologic impacts of climate change. Current Climate Change Reports, 2(2), 55-64.

Cleveland, W. S. (1979). Robust locally weighted regression and smoothing scatterplots. Journal of the American Statistical Association, 74(368), 829-836.

Climate Data. (2009). Guidelines on analysis of extremes in a changing climate in support of informed decisions for adaptation. World Meteorological Organization. 
Coe, R., \& Stern, R. D. (1982). Fitting models to daily rainfall data. Journal of Applied Meteorology, 21(7), 1024-1031.

Coles, S. G., \& Tawn, J. A. (1996). A Bayesian analysis of extreme rainfall data. Journal of the Royal Statistical Society: Series C (Applied Statistics), 45(4), 463-478.

Colman, R. (2003). A comparison of climate feedbacks in general circulation models. Climate Dynamics, 20(7-8), 865-873.

Costache, R. (2019). Flash-flood Potential Index mapping using weights of evidence, decision Trees models and their novel hybrid integration. Stochastic Environmental Research and Risk Assessment, 33(7), 1375-1402.

Couasnon, A., Scussolini, P., Van Thu Tran, T., Eilander, D., Muis, S., Wang, H., Keesom, J., Dullaart, J., Xuan, Y., NguyenHong, Q., Winsemius, H., \& Ward, P. J. (2021). Seasonal Dependence between Rainfall and Sea Levels Modulates Flood Risk in Ho Chi Minh City. Water resources research (Under review).

Coughlan de Perez, E. R., Van den Hurk, B., Van Aalst, M. K., Amuron, I., Bamanya, D., Hauser, T., Jongma, B., Lopez, A., Mason, S. J., \& Mendler de Suarez, J. (2016). Action-based flood forecasting for triggering humanitarian action.

Covey, C., AchutaRao, K. M., Cubasch, U., Jones, P., Lambert, S. J., Mann, M. E., Phillips, T. J., \& Taylor, K. E. (2003). An overview of results from the Coupled Model Intercomparison Project. Global and Planetary Change, 37(1-2), 103-133.

Cressie, N., \& Hawkins, D. M. (1980). Robust estimation of the variogram: I. Journal of the International Association for Mathematical Geology, 12(2), 115-125.

CSIRO, \& Meteorology, t. A. B. o. (2018). Previous State of the Climate reports. Retrieved from https://www.csiro.au/en/Research/OandA/Areas/Assessing-our-climate/State-ofthe-Climate-2018/Previous-State-of-the-Climate-reports

D'Amico, G., \& Petroni, F. (2018). Copula based multivariate semi-Markov models with applications in high-frequency finance. European Journal of Operational Research, 267(2), 765-777.

Dadson, S. J., Lopez, H. P., Peng, J., \& Vora, S. (2019). Hydroclimatic Extremes and Climate Change. Water Science, Policy, and Management: A Global Challenge, 11-28.

de Leeuw, J., Methven, J., \& Blackburn, M. (2016). Variability and trends in England and Wales precipitation. International Journal of Climatology, 36(8), 2823-2836.

De Luca, P., Messori, G., Wilby, R. L., Mazzoleni, M., \& Di Baldassarre, G. (2020). Concurrent wet and dry hydrological extremes at the global scale. Earth System Dynamics, 11(1), 251-266.

de Winter, J. C., Gosling, S. D., \& Potter, J. (2016). Comparing the Pearson and Spearman correlation coefficients across distributions and sample sizes: A tutorial using simulations and empirical data. Psychological Methods, 21(3), 273.

Di Luca, A., Pitman, A. J., \& de Elía, R. (2020). Decomposing temperature extremes errors in CMIP5 and CMIP6 models. Geophysical Research Letters, 47(14), e2020GL088031.

Dias, A., \& Embrechts, P. (2004). Dynamic copula models for multivariate high-frequency data in finance. Manuscript, ETH Zurich, 81. 
Diaz, V., Perez, G. A. C., Van Lanen, H. A., Solomatine, D., \& Varouchakis, E. A. (2020). An approach to characterise spatio-temporal drought dynamics. Advances in Water Resources, 137, 103512.

Drusch, M., Wood, E. F., Gao, H., \& Thiele, A. (2004). Soil moisture retrieval during the Southern Great Plains Hydrology Experiment 1999: A comparison between experimental remote sensing data and operational products. Water Resources Research, 40(2).

Du, J., Cheng, L., \& Zhang, Q. (2019). Spatiotemporal variability and trends in the hydrology of the Xiang River basin, China: extreme precipitation and streamflow. Arabian journal of geosciences, 12(18), 566.

Duane, W., Livingstone, D., \& Kidd, D. (2000). Integrating environmental models with GIS: an object - oriented approach utilising a hierarchical data format (HDF) data repository. Transactions in GIS, 4(3), 263-280.

Eduardo, G. (2020, 2021-01-19). Notes for Nonparametric Statistics. Chapter 6 Nonparametric tests. Retrieved from https://bookdown.org/egarpor/NP-UC3M/nptests-gofdist.html\#nptests-gof-dist-boot

Efron, B., \& Tibshirani, R. (1997). Improvements on cross-validation: the 632+ bootstrap method. Journal of the American Statistical Association, 92(438), 548-560.

Embrechts, P., Lindskog, F., \& McNeil, A. (2001). Modelling dependence with copulas. Rapport technique, Département de mathématiques, Institut Fédéral de Technologie de Zurich, Zurich, 14.

Emura, T., Shih, J.-H., Ha, I. D., \& Wilke, R. A. (2020). Comparison of the marginal hazard model and the sub-distribution hazard model for competing risks under an assumed copula. Statistical Methods in Medical Research, 29(8), 2307-2327.

Eyring, V., Bony, S., Meehl, G. A., Senior, C. A., Stevens, B., Stouffer, R. J., \& Taylor, K. E. (2016). Overview of the Coupled Model Intercomparison Project Phase 6 (CMIP6) experimental design and organization. Geoscientific Model Development, 9(5), 19371958.

Fairman Jr, J. G., Schultz, D. M., Kirshbaum, D. J., Gray, S. L., \& Barrett, A. I. (2017). Climatology of size, shape, and intensity of precipitation features over Great Britain and Ireland. Journal of Hydrometeorology, 18(6), 1595-1615.

Fan, J. (1993). Local linear regression smoothers and their minimax efficiencies. The annals of statistics, 196-216.

Fasano, G., \& Franceschini, A. (1987). A multidimensional version of the KolmogorovSmirnov test. Monthly Notices of the Royal Astronomical Society, 225(1), 155-170.

Fathian, F., Dehghan, Z., Bazrkar, M. H., \& Eslamian, S. (2016). Trends in hydrological and climatic variables affected by four variations of the Mann-Kendall approach in Urmia Lake basin, Iran. Hydrological Sciences Journal, 61(5), 892-904.

Favre, A. C., El Adlouni, S., Perreault, L., Thiémonge, N., \& Bobée, B. (2004). Multivariate hydrological frequency analysis using copulas. Water Resources Research, 40(1). 
Fedorov, A. V., Hu, S., Lengaigne, M., \& Guilyardi, E. (2015). The impact of westerly wind bursts and ocean initial state on the development, and diversity of El Niño events. Climate Dynamics, 44(5-6), 1381-1401.

Feng, S., Nadarajah, S., \& Hu, Q. (2007). Modeling annual extreme precipitation in China using the generalized extreme value distribution. Journal of the Meteorological Society of Japan. Ser. II, 85(5), 599-613.

Feng, Y., Shi, P., Qu, S., Mou, S., Chen, C., \& Dong, F. (2020). Nonstationary flood coincidence risk analysis using time-varying copula functions. Scientific Reports, $10(1), 1-12$.

Field, C. B., Barros, V., Stocker, T. F., \& Dahe, Q. (2012). Managing the risks of extreme events and disasters to advance climate change adaptation: special report of the intergovernmental panel on climate change: Cambridge University Press.

Fischer, E. M., \& Knutti, R. (2014). Detection of spatially aggregated changes in temperature and precipitation extremes. Geophysical Research Letters, 41(2), 547-554.

Flanagan, J., Davies, G. H., Boy, F., \& Doneddu, D. (2020). A Review of a Distributed High performance Computing Implementation. Journal of Information Technology Case and Application Research, 22(3), 142-158.

Fox, J. (2009). A Monte Carlo Approach to Joint Probability of Wave, Tide, and Surge in Extreme Water Level Calculations. Health and Safety Executive, Research Report, 740.

Friedman, J., Hastie, T., \& Tibshirani, R. (2001). The elements of statistical learning (Vol. 1): Springer series in statistics New York.

Ganguli, P., \& Coulibaly, P. (2017). Does nonstationarity in rainfall require nonstationary intensity-duration-frequency curves? Hydrology and Earth System Sciences, 21(12), 6461-6483.

Gelman, A., \& Rubin, D. B. (1992). Inference from iterative simulation using multiple sequences. Statistical science, 7(4), 457-472.

Gentine, P., Pritchard, M., Rasp, S., Reinaudi, G., \& Yacalis, G. (2018). Could machine learning break the convection parameterization deadlock? Geophysical Research Letters, 45(11), 5742-5751.

Gilks, W. R., Best, N. G., \& Tan, K. (1995). Adaptive rejection Metropolis sampling within Gibbs sampling. Journal of the Royal Statistical Society: Series C (Applied Statistics), 44(4), 455-472.

Giorgetta, M. A., Jungclaus, J., Reick, C. H., Legutke, S., Bader, J., Böttinger, M., Brovkin, V., Crueger, T., Esch, M., \& Fieg, K. (2013). Climate and carbon cycle changes from 1850 to 2100 in MPI - ESM simulations for the Coupled Model Intercomparison Project phase 5. Journal of Advances in Modeling Earth Systems, 5(3), 572-597.

Golding, B. (1998). Nimrod: A system for generating automated very short range forecasts. Meteorological Applications: A journal of forecasting, practical applications, training techniques and modelling, 5(1), 1-16. 
Gori, A., Lin, N., \& Smith, J. (2020). Assessing compound flooding from landfalling tropical cyclones on the North Carolina coast. Water Resources Research, 56(4), e2019WR026788.

Graf, H. F., \& Zanchettin, D. (2012). Central Pacific El Niño, the "subtropical bridge," and Eurasian climate. Journal of Geophysical Research: Atmospheres, 117(D1).

Grzymala-Busse, J. W. (1993). Selected algorithms of machine learning from examples. Fundam. Inform., 18, 193-207.

Guenzi, D., Fratianni, S., Boraso, R., \& Cremonini, R. (2017). CondMerg: an open source implementation in $\mathrm{R}$ language of conditional merging for weather radars and rain gauges observations. Earth Science Informatics, 10(1), 127-135.

Güneralp, B., Güneralp, İ., \& Liu, Y. (2015). Changing global patterns of urban exposure to flood and drought hazards. Global Environmental Change, 31, 217-225.

Guo, L., \& Ljung, L. (1995). Performance analysis of general tracking algorithms. IEEE Transactions on Automatic Control, 40(8), 1388-1402.

Gusain, A., Ghosh, S., \& Karmakar, S. (2020). Added value of CMIP6 over CMIP5 models in simulating Indian summer monsoon rainfall. Atmospheric Research, 232, 104680.

Haghnegahdar, A., Razavi, S., Yassin, F., \& Wheater, H. (2017). Multicriteria sensitivity analysis as a diagnostic tool for understanding model behaviour and characterizing model uncertainty. Hydrological Processes, 31(25), 4462-4476.

Hall, J., Arheimer, B., Borga, M., Brázdil, R., Claps, P., Kiss, A., Kjeldsen, T., Kriauciuniene, J., Kundzewicz, Z., \& Lang, M. (2014). Understanding flood regime changes in Europe: A state of the art assessment.

Hally, D. (1987). Calculation of the Moments of Polygons. Retrieved from

Hasan, H., Radi, N. F. A., \& Kassim, S. (2012). Modeling of extreme temperature using generalized extreme value (GEV) distribution: A case study of Penang.

He, T., Einfalt, T., Zhang, J., Hua, J., \& Cai, Y. (2019). New Algorithm for Rain Cell Identification and Tracking in Rainfall Event Analysis. Atmosphere, 10(9), 532.

Hendry, A., Haigh, I. D., Nicholls, R. J., Winter, H., Neal, R., Wahl, T., Joly-Laugel, A., \& Darby, S. E. (2019). Assessing the characteristics and drivers of compound flooding events around the UK coast. Hydrology and Earth System Sciences, 23(7), 3117-3139.

Her, Y., Yoo, S.-H., Cho, J., Hwang, S., Jeong, J., \& Seong, C. (2019). Uncertainty in hydrological analysis of climate change: multi-parameter vs. multi-GCM ensemble predictions. Scientific Reports, 9(1), 1-22.

Herring, S. C., Christidis, N., Hoell, A., Kossin, J. P., Schreck Iii, C. J., \& Stott, P. A. (2018). Explaining extreme events of 2016 from a climate perspective. Bulletin of the American Meteorological Society, 99(1), S1-S157.

Hersbach, H., Peubey, C., Simmons, A., Berrisford, P., Poli, P., \& Dee, D. (2015). ERA 20CM: A twentieth - century atmospheric model ensemble. Quarterly Journal of the Royal Meteorological Society, 141(691), 2350-2375. 
Hervouet, J. M. (2000). TELEMAC modelling system: an overview. Hydrological Processes, 14(13), 2209-2210.

Hirabayashi, Y., Mahendran, R., Koirala, S., Konoshima, L., Yamazaki, D., Watanabe, S., Kim, H., \& Kanae, S. (2013). Global flood risk under climate change. Nature Climate Change, 3(9), 816-821.

Hoi, H. T. (2020). Impacts of Urbanization on the Environment of Ho Chi Minh City. Paper presented at the IOP Conference Series: Earth and Environmental Science.

Hong, H., Liu, J., Bui, D. T., Pradhan, B., Acharya, T. D., Pham, B. T., Zhu, A.-X., Chen, W., \& Ahmad, B. B. (2018). Landslide susceptibility mapping using J48 Decision Tree with AdaBoost, Bagging and Rotation Forest ensembles in the Guangchang area (China). Catena, 163, 399-413.

Hosking, J. R. (1990). L - moments: Analysis and estimation of distributions using linear combinations of order statistics. Journal of the Royal Statistical Society: Series B (Methodological), 52(1), 105-124.

Hu, C., Yang, S., Wu, Q., Li, Z., Chen, J., Deng, K., Zhang, T., \& Zhang, C. (2016). Shifting El Niño inhibits summer Arctic warming and Arctic sea-ice melting over the Canada Basin. Nature communications, 7(1), 1-9.

Huang, T. S. (1996). Computer vision: Evolution and promise. CERN European Organization for Nuclear Research-Reports-CERN, 21-26.

Im, E.-S., Ahn, J.-B., \& Jo, S.-R. (2015). Regional climate projection over South Korea simulated by the HadGEM2-AO and WRF model chain under RCP emission scenarios. Climate Research, 63(3), 249-266.

Inglezakis, V., Poulopoulos, S., Arkhangelsky, E., Zorpas, A., \& Menegaki, A. (2016). Aquatic environment. In Environment and Development (pp. 137-212): Elsevier.

Inoue, A., Jin, L., \& Rossi, B. (2017). Rolling window selection for out-of-sample forecasting with time-varying parameters. Journal of Econometrics, 196(1), 55-67.

IPCC. (2021). Climate Change 2021: The Physical Science Basis.: Cambridge University Press. In Press.

IRDR. (2013). Integrated Research on Disaster Risk Strategic Plan 2013-2017. Beijing

ISCU. (2008). A science plan for integrated research on disaster risk: Addressing the challenge of natural and human-induced environmental hazards. Paris, France

Islam, T., Rico-Ramirez, M. A., Srivastava, P. K., \& Dai, Q. (2014). Non-parametric rain/no rain screening method for satellite-borne passive microwave radiometers at $19-85 \mathrm{GHz}$ channels with the Random Forests algorithm. International Journal of Remote Sensing, 35(9), 3254-3267.

Izaguirre, C., Mendez, F. J., Menendez, M., Luceño, A., \& Losada, I. J. (2010). Extreme wave climate variability in southern Europe using satellite data. Journal of Geophysical Research: Oceans, 115(C4).

James, G. M. (2002). Generalized linear models with functional predictors. Journal of the Royal Statistical Society: Series B (Statistical Methodology), 64(3), 411-432. 
Jebson, S. (2007). Fact sheet number 3: Water in the atmosphere.

Jenkinson, A. F. (1955). The frequency distribution of the annual maximum (or minimum) values of meteorological elements. Quarterly Journal of the Royal Meteorological Society, 81(348), 158-171.

Jha, A. K., Bloch, R., \& Lamond, J. (2012). Cities and flooding: a guide to integrated urban flood risk management for the 21st century: The World Bank.

Jhong, B.-C., \& Tung, C.-P. (2018). Evaluating future joint probability of precipitation extremes with a copula-based assessing approach in climate change. Water Resources Management, 32(13), 4253-4274.

Jiang, F., Zhang, W., Geng, X., Stuecker, M. F., \& Liu, C. (2019). Impacts of central Pacific El Niño on southern China spring precipitation controlled by its longitudinal position. Journal of Climate, 32(22), 7823-7836.

Jiang, S., Zheng, Y., Babovic, V., Tian, Y., \& Han, F. (2018). A computer vision-based approach to fusing spatiotemporal data for hydrological modeling. Journal of Hydrology, 567, 25-40.

Jones, D. A., Wang, W., \& Fawcett, R. (2009). High-quality spatial climate data-sets for Australia. Australian Meteorological and Oceanographic Journal, 58(4), 233.

Jonkman, S. N. (2005). Global perspectives on loss of human life caused by floods. Natural Hazards, 34(2), 151-175.

Joslyn, S. L., \& LeClerc, J. E. (2012). Uncertainty forecasts improve weather-related decisions and attenuate the effects of forecast error. Journal of Experimental Psychology: Applied, 18(1), 126.

Jovanovic, S., Skoric, T., Sarenac, O., Milutinovic-Smiljanic, S., Japundzic-Zigon, N., \& Bajic, D. (2018). Copula as a dynamic measure of cardiovascular signal interactions. Biomedical Signal Processing and Control, 43, 250-264.

Kahneman, D., \& Tversky, A. (1979). On the interpretation of intuitive probability: A reply to Jonathan Cohen.

Kantar, Y. M., \& Şenoğlu, B. (2008). A comparative study for the location and scale parameters of the Weibull distribution with given shape parameter. Computers \& Geosciences, 34(12), 1900-1909.

Kass, M., Witkin, A., \& Terzopoulos, D. (1988). Snakes: Active contour models. International Journal of Computer Vision, 1(4), 321-331.

Kenabatho, P., Parida, B., \& Moalafhi, D. (2017). Evaluation of satellite and simulated rainfall products for hydrological applications in the Notwane Catchment, Botswana. Physics and Chemistry of the Earth, Parts $A / B / C, 100,19-30$.

Kendall, M. G. (1948). Rank correlation methods.

Kendon, M., MacCarthy, M., \& Jevrejeva, S. (2015). State of the UK Climate 2014: Met Office.

Kendon, M., McCarthy, M., Jevrejeva, S., Matthews, A., \& Legg, T. (2018). State of the UK climate 2017. International Journal of Climatology, 38, 1-35. 
Kendon, M., McCarthy, M., Jevrejeva, S., Matthews, A., \& Legg, T. (2019). State of the UK climate 2018. International Journal of Climatology, 39, 1-55.

Kidd, C., \& Huffman, G. (2011). Global precipitation measurement. Meteorological Applications, 18(3), 334-353.

Kim, H., Kim, S., Shin, H., \& Heo, J.-H. (2017). Appropriate model selection methods for nonstationary generalized extreme value models. Journal of Hydrology, 547, 557-574.

Kim, H., Yeh, S. W., An, S. I., Park, J. H., Kim, B. M., \& Baek, E. H. (2020). Arctic Sea ice loss as a potential trigger for Central Pacific El Niño events. Geophysical Research Letters, 47(7), e2020GL087028.

Kim, S. T., \& Yu, J. Y. (2012). The two types of ENSO in CMIP5 models. Geophysical Research Letters, 39(11).

Kirono, D. G., Chiew, F. H., \& Kent, D. M. (2010). Identification of best predictors for forecasting seasonal rainfall and runoff in Australia. Hydrological Processes: An International Journal, 24(10), 1237-1247.

Kolmogorov, A. (1933). Sulla determinazione empirica di una lgge di distribuzione. Inst. Ital. Attuari, Giorn., 4, 83-91.

Kontgis, C., Schneider, A., Fox, J., Saksena, S., Spencer, J. H., \& Castrence, M. (2014). Monitoring peri-urbanization in the greater Ho Chi Minh City metropolitan area. Applied Geography, 53, 377-388.

Krizhevsky, A., Sutskever, I., \& Hinton, G. E. (2012). Imagenet classification with deep convolutional neural networks. Advances in Neural Information Processing Systems, $25,1097-1105$.

Kuchment, L. S. (2004). The hydrological cycle and human impact on it. Water Resources Management, 40.

Kumbier, K., Carvalho, R. C., Vafeidis, A. T., \& Woodroffe, C. D. (2018). Investigating compound flooding in an estuary using hydrodynamic modelling: a case study from the Shoalhaven River, Australia. Natural Hazards and Earth System Sciences, 18(2), 463477.

Kundzewicz, Z. W., \& Robson, A. J. (2004). Change detection in hydrological records-a review of the methodology/revue méthodologique de la détection de changements dans les chroniques hydrologiques. Hydrological Sciences Journal, 49(1), 7-19.

Kwon, H. H., \& Lall, U. (2016). A copula - based nonstationary frequency analysis for the 2012 - 2015 drought in California. Water Resources Research, 52(7), 5662-5675.

Larkin, N. K., \& Harrison, D. (2005). Global seasonal temperature and precipitation anomalies during El Niño autumn and winter. Geophysical Research Letters, 32(16).

Laverde-Barajas, M., Perez, G. C., Chishtie, F., Poortinga, A., Uijlenhoet, R., \& Solomatine, D. (2020). Decomposing satellite-based rainfall errors in flood estimation: Hydrological responses using a spatiotemporal object-based verification method. Journal of Hydrology, 591, 125554.

Lazoglou, G., \& Anagnostopoulou, C. (2017). An Overview of Statistical Methods for Studying the Extreme Rainfalls in Mediterranean. 
Leadbetter, M. R. (1991). On a basis for 'Peaks over Threshold'modeling. Statistics \& Probability Letters, 12(4), 357-362.

Lehner, B., Döll, P., Alcamo, J., Henrichs, T., \& Kaspar, F. (2006). Estimating the impact of global change on flood and drought risks in Europe: a continental, integrated analysis. Climatic Change, 75(3), 273-299.

Lehner, F., Wood, A. W., Vano, J. A., Lawrence, D. M., Clark, M. P., \& Mankin, J. S. (2019). The potential to reduce uncertainty in regional runoff projections from climate models. Nature Climate Change, 9(12), 926-933.

Levoy, M., \& Hanrahan, P. (1996). Light field rendering. Paper presented at the Proceedings of the 23rd annual conference on Computer graphics and interactive techniques.

Li, Z., Li, Z., Zhao, W., \& Wang, Y. (2015). Probability modeling of precipitation extremes over two river basins in northwest of China. Advances in Meteorology, 2015.

Lilliefors, H. W. (1967). On the Kolmogorov-Smirnov test for normality with mean and variance unknown. Journal of the American Statistical Association, 62(318), 399-402.

Lin, F.-R., Wu, N.-J., \& Tsay, T.-K. (2017). Applications of cluster analysis and pattern recognition for typhoon hourly rainfall forecast. Advances in Meteorology, 2017.

Liu, Q. Y., Wang, D., Wang, X., Shu, Y., Xie, Q., \& Chen, J. (2014). Thermal variations in the $\mathrm{S}$ outh $\mathrm{C}$ hina $\mathrm{S}$ ea associated with the eastern and central $\mathrm{P}$ acific $\mathrm{E} 1 \mathrm{~N}$ iño events and their mechanisms. Journal of Geophysical Research: Oceans, 119(12), 8955-8972.

Liu, Y., Racah, E., Correa, J., Khosrowshahi, A., Lavers, D., Kunkel, K., Wehner, M., \& Collins, W. (2016). Application of deep convolutional neural networks for detecting extreme weather in climate datasets. arXiv preprint arXiv:1605.01156.

Ljungqvist, F. C., Krusic, P. J., Sundqvist, H. S., Zorita, E., Brattström, G., \& Frank, D. (2016). Northern Hemisphere hydroclimate variability over the past twelve centuries. Nature, 532(7597), 94-98.

Lobligeois, F., Andréassian, V., Perrin, C., Tabary, P., \& Loumagne, C. (2014). When does higher spatial resolution rainfall information improve streamflow simulation? An evaluation using 3620 flood events.

Lowe, J. A., Bernie, D., Bett, P., Bricheno, L., Brown, S., Calvert, D., Clark, R., Eagle, K., Edwards, T., \& Fosser, G. (2018). UKCP18 science overview report. Met Office Hadley Centre: Exeter, UK.

Lowe, J. A., Bernie, D., Bett, P., Bricheno, L., Brown, S., Calvert, D., Clark, R., Eagle, K., Edwards, T., \& Fosser, G. (2018). UKCP18 science overview report. Exeter, UK: Met Office Hadley Centre.

Manly, B. F. J. (2018). Randomization, bootstrap and Monte Carlo methods in biology: Chapman and Hall/CRC.

Mann, H. B. (1945). Nonparametric tests against trend. Econometrica: Journal of the Econometric Society, 245-259.

Mann, N. R. (1967). Results on location and scale parameter estimation with application to the extreme-value distribution. In: ROCKETDYNE CANOGA PARK CA. 
Mannshardt-Shamseldin, C., E., Smith, R. L., Sain, S. R., Mearns, L. O., \& Cooley, D. (2010). Downscaling extremes: A comparison of extreme value distributions in point-source and gridded precipitation data. The Annals of Applied Statistics, 4(1), 484-502.

Mannshardt-Shamseldin, E. C., Smith, R. L., Sain, S. R., Mearns, L. O., \& Cooley, D. (2010). Downscaling extremes: A comparison of extreme value distributions in point-source and gridded precipitation data. The Annals of Applied Statistics, 4(1), 484-502.

Maraun, D., Wetterhall, F., Ireson, A., Chandler, R., Kendon, E., Widmann, M., Brienen, S., Rust, H., Sauter, T., \& Themeß1, M. (2010). Precipitation downscaling under climate change: Recent developments to bridge the gap between dynamical models and the end user. Reviews of Geophysics, 48(3).

Martins, E. S., \& Stedinger, J. R. (2000). Generalized maximum - likelihood generalized extreme - value quantile estimators for hydrologic data. Water Resources Research, 36(3), 737-744.

McCullagh, P. (1989). Generalized linear models: Routledge.

McCullagh, P. (2018). Generalized linear models: Routledge.

Meehl, G. A., Stocker, T. F., Collins, W. D., Friedlingstein, P., Gaye, A. T., Gregory, J. M., Kitoh, A., Knutti, R., Murphy, J. M., \& Noda, A. (2007). Global climate projections. Chapter 10.

Mehta, B. B., Ma, D., Pierre, E. Y., Jiang, Y., Coppo, S., \& Griswold, M. A. (2018). Image reconstruction algorithm for motion insensitive MR Fingerprinting (MRF): MORF. Magnetic Resonance in Medicine, 80(6), 2485-2500.

Meresa, H. K., \& Romanowicz, R. J. (2017). The critical role of uncertainty in projections of hydrological extremes. Hydrology and Earth System Sciences, 21(8), 4245.

Metropolis, N., \& Ulam, S. (1949). The monte carlo method. Journal of the American Statistical Association, 44(247), 335-341.

Milgram, D. (1979). Region extraction using convergent evidence. Computer Graphics and Image Processing, 11(1), 1-12.

Millán, M. M., Estrela, M. J., \& Miró, J. (2005). Rainfall components: variability and spatial distribution in a Mediterranean Area (Valencia Region). Journal of Climate, 18(14), 2682-2705.

Milly, P. C. D., Wetherald, R. T., Dunne, K., \& Delworth, T. L. (2002). Increasing risk of great floods in a changing climate. Nature, 415(6871), 514-517.

Miniussi, A., Marani, M., \& Villarini, G. (2020). Metastatistical Extreme Value Distribution applied to floods across the continental United States. Advances in Water Resources, 136, 103498.

MNRE. (2009). Climate change, sea level rise scenarios for Vietnam.

Moftakhari, H. R., Salvadori, G., AghaKouchak, A., Sanders, B. F., \& Matthew, R. A. (2017). Compounding effects of sea level rise and fluvial flooding. Proceedings of the National Academy of Sciences, 114(37), 9785-9790. 
Monteiro, J. A., Strauch, M., Srinivasan, R., Abbaspour, K., \& Gücker, B. (2016). Accuracy of grid precipitation data for Brazil: application in river discharge modelling of the Tocantins catchment. Hydrological Processes, 30(9), 1419-1430.

Moran, G. W. (1984). Locally-Weighted-Regression Scatter-Plot Smoothing (LOWESS): a graphical exploratory data analysis technique. Retrieved from

Morissette, L., \& Chartier, S. (2013). The k-means clustering technique: General considerations and implementation in Mathematica. Tutorials in Quantitative Methods for Psychology, 9(1), 15-24.

Morrison, J. E., \& Smith, J. A. (2002). Stochastic modeling of flood peaks using the generalized extreme value distribution. Water Resources Research, 38(12), 41-41-41-12.

Moufouma-Okia, W., \& Jones, R. (2015). Resolution dependence in simulating the African hydroclimate with the HadGEM3-RA regional climate model. Climate Dynamics, 44(3-4), 609-632.

Mulder, K. J., Lickiss, M., Black, A., Charlton - Perez, A. J., McCloy, R., \& Young, J. S. (2020). Designing environmental uncertainty information for experts and non experts: Does data presentation affect users decisions and interpretations? Meteorological Applications, 27(1), e1821.

Murphy, A. H. (1976). Decision-making models in the cost-loss ratio situation and measures of the value of probability forecasts. Monthly Weather Review, 104(8), 1058-1065.

Murphy, A. H. (1977). The value of climatological, categorical and probabilistic forecasts in the cost-loss ratio situation. Monthly Weather Review, 105(7), 803-816.

Muthusamy, M., Schellart, A., Tait, S., \& Heuvelink, G. (2017a). Geostatistical upscaling of rain gauge data to support uncertainty analysis of lumped urban hydrological models. Hydrology and Earth System Sciences, 21(2), 1077-1091.

Muthusamy, M., Schellart, A., Tait, S., \& Heuvelink, G. B. (2017b). Geostatistical upscaling of rain gauge data to support uncertainty analysis of lumped urban hydrological models. Hydrology and Earth System Sciences, 21(2), 1077-1091.

Myung, I. J. (2003). Tutorial on maximum likelihood estimation. Journal of Mathematical Psychology, 47(1), 90-100.

Nashwan, M. S., Shahid, S., \& Chung, E.-S. (2019). Development of high-resolution daily gridded temperature datasets for the central north region of Egypt. Scientific data, 6(1), $1-13$.

Nayak, M. A., \& Ghosh, S. (2013). Prediction of extreme rainfall event using weather pattern recognition and support vector machine classifier. Theoretical and Applied Climatology, 114(3), 583-603.

Neale, R. B., Richter, J., Park, S., Lauritzen, P. H., Vavrus, S. J., Rasch, P. J., \& Zhang, M. (2013). The mean climate of the Community Atmosphere Model (CAM4) in forced SST and fully coupled experiments. Journal of Climate, 26(14), 5150-5168.

NERC. (1977). Flood studies supplementary report No 1: The areal reduction factor in rainfall frequency estimation. UK: Natural Environment Research Council. 
Nguyen-Le, D., \& Yamada, T. J. (2019). Using weather pattern recognition to classify and predict summertime heavy rainfall occurrence over the Upper Nan river basin, northwestern Thailand. Weather and Forecasting, 34(2), 345-360.

Ning, S., Wang, J., Jin, J., \& Ishidaira, H. (2016). Assessment of the latest GPM-Era highresolution satellite precipitation products by comparison with observation gauge data over the Chinese mainland. Water, 8(11), 481.

O'Gorman, P. A., \& Dwyer, J. G. (2018). Using machine learning to parameterize moist convection: Potential for modeling of climate, climate change, and extreme events. Journal of Advances in Modeling Earth Systems, 10(10), 2548-2563.

Ochoa-Rodriguez, S., Wang, L.-P., Gires, A., Pina, R. D., Reinoso-Rondinel, R., Bruni, G., Ichiba, A., Gaitan, S., Cristiano, E., \& van Assel, J. (2015). Impact of spatial and temporal resolution of rainfall inputs on urban hydrodynamic modelling outputs: A multi-catchment investigation. Journal of Hydrology, 531, 389-407.

Office, M. Annual report and account. Retrieved from

Önöz, B., \& Bayazit, M. (2012). Block bootstrap for Mann-Kendall trend test of serially dependent data. Hydrological Processes, 26(23), 3552-3560.

Overeem, A., Buishand, T. A., Holleman, I., \& Uijlenhoet, R. (2010). Extreme value modeling of areal rainfall from weather radar. Water Resources Research, 46(9).

Panagoulia, D., Economou, P., \& Caroni, C. (2014). Stationary and nonstationary generalized extreme value modelling of extreme precipitation over a mountainous area under climate change. Environmetrics, 25(1), 29-43.

Panu, U. S., Unny, T., \& Ragade, R. (1978). A feature prediction model in synthetic hydrology based on concepts of pattern recognition. Water Resources Research, 14(2), 335-344.

Paolanti, M., \& Frontoni, E. (2020). Multidisciplinary pattern recognition applications: A review. Computer Science Review, 37, 100276.

Pappenberger, F., Iorgulescu, I., \& Beven, K. J. (2006). Sensitivity analysis based on regional splits and regression trees (SARS-RT). Environmental Modelling and Software, 21(7), 976-990.

Parchami, M., Amindavar, H., \& Zhu, W.-P. (2020). Learning-based design of random measurement matrix for compressed sensing with inter-column correlation using copula function. IET Signal Processing, 14(6), 385-395.

Parkes, B., \& Demeritt, D. (2016). Defining the hundred year flood: A Bayesian approach for using historic data to reduce uncertainty in flood frequency estimates. Journal of Hydrology, 540, 1189-1208.

Pavlidis, T., \& Liow, Y.-T. (1990). Integrating region growing and edge detection. IEEE Transactions on Pattern Analysis and Machine Intelligence, 12(3), 225-233.

Pedamonti, D. (2018). Comparison of non-linear activation functions for deep neural networks on MNIST classification task. arXiv preprint arXiv:1804.02763.

Peleg, N., Marra, F., Fatichi, S., Paschalis, A., Molnar, P., \& Burlando, P. (2018). Spatial variability of extreme rainfall at radar subpixel scale. Journal of Hydrology, 556, 922933. 
Petersik, P. J., \& Dijkstra, H. A. (2020). Probabilistic Forecasting of El Niño Using Neural Network Models. Geophysical Research Letters, 47(6), e2019GL086423.

Pham, D. T., Dimov, S. S., \& Nguyen, C. D. (2005). Selection of K in K-means clustering. Proceedings of the Institution of Mechanical Engineers, Part C: Journal of Mechanical Engineering Science, 219(1), 103-119.

Pierce, D. A., \& Schafer, D. W. (1986). Residuals in generalized linear models. Journal of the American Statistical Association, 81(396), 977-986.

Poelman, C. J., \& Kanade, T. (1997). A paraperspective factorization method for shape and motion recovery. IEEE Transactions on Pattern Analysis and Machine Intelligence, 19(3), 206-218.

Prein, A. F., Rasmussen, R. M., Ikeda, K., Liu, C., Clark, M. P., \& Holland, G. J. (2017). The future intensification of hourly precipitation extremes. Nature Climate Change, 7(1), 48.

Qi, W., Zhang, C., Fu, G., Zhou, H., \& Liu, J. (2016). Quantifying uncertainties in extreme flood predictions under climate change for a medium-sized basin in Northeastern China. Journal of Hydrometeorology, 17(12), 3099-3112.

Qiu, M., Zhao, P., Zhang, K., Huang, J., Shi, X., Wang, X., \& Chu, W. (2017). A short-term rainfall prediction model using multi-task convolutional neural networks. Paper presented at the 2017 IEEE International Conference on Data Mining (ICDM).

Quinlan, J. R. (1986). Induction of decision trees. Machine Learning, 1(1), 81-106.

Quinn, T., Bousquet, F., Guerbois, C., Heider, L., \& Brown, K. (2019). How local water and waterbody meanings shape flood risk perception and risk management preferences. Sustainability Science, 14(3), 565-578.

Rabiei, E., \& Haberlandt, U. (2015). Applying bias correction for merging rain gauge and radar data. Journal of Hydrology, 522, 544-557.

Ragulina, G., \& Reitan, T. (2017). Generalized extreme value shape parameter and its nature for extreme precipitation using long time series and the Bayesian approach. Hydrological Sciences Journal, 62(6), 863-879.

Ramachandra, V. (2019). Causal inference for climate change events from satellite image time series using computer vision and deep learning. arXiv preprint arXiv:1910.11492.

Ramirez-Villegas, J., Challinor, A. J., Thornton, P. K., \& Jarvis, A. (2013). Implications of regional improvement in global climate models for agricultural impact research. Environmental Research Letters, 8(2), 024018.

Ramos, M. H., Van Andel, S. J., \& Pappenberger, F. (2013). Do probabilistic forecasts lead to better decisions?

Randall, D. A., Wood, R. A., Bony, S., Colman, R., Fichefet, T., Fyfe, J., Kattsov, V., Pitman, A., Shukla, J., \& Srinivasan, J. (2007). Climate models and their evaluation. In Climate change 2007: The physical science basis. Contribution of Working Group I to the Fourth Assessment Report of the IPCC (FAR) (pp. 589-662): Cambridge University Press. 
Rashid, M., Beecham, S., Chowdhury, R., Piantadosi, J., Anderssen, R. S., \& Boland, J. (2013). Simulation of extreme rainfall from CMIP5 in the Onkaparinga catchment using a generalized linear model.

Rasmussen, C. E., \& Ghahramani, Z. (2003). Bayesian monte carlo. Paper presented at the Advances in neural information processing systems (conference).

Ratnam, J., Behera, S., Masumoto, Y., \& Yamagata, T. (2014). Remote effects of El Niño and Modoki events on the austral summer precipitation of southern Africa. Journal of Climate, 27(10), 3802-3815.

Renard, B., \& Lang, M. (2007). Use of a Gaussian copula for multivariate extreme value analysis: some case studies in hydrology. Advances in Water Resources, 30(4), 897912.

Rew, R., \& Davis, G. (1990). NetCDF: an interface for scientific data access. IEEE computer graphics and applications, 10(4), 76-82.

Ricke, K. L., Morgan, M. G., \& Allen, M. R. (2010). Regional climate response to solarradiation management. Nature Geoscience, 3(8), 537-541.

Roberts, G. O., \& Rosenthal, J. S. (2009). Examples of adaptive MCMC. Journal of Computational and Graphical Statistics, 18(2), 349-367.

Rogger, M., Agnoletti, M., Alaoui, A., Bathurst, J. C., Bodner, G., Borga, M., Chaplot, V., Gallart, F., Glatzel, G., \& Hall, J. (2017). Land use change impacts on floods at the catchment scale: Challenges and opportunities for future research. Water Resources Research, 53(7), 5209-5219.

Rojas, R., Feyen, L., Dosio, A., \& Bavera, D. (2011). Improving pan-European hydrological simulation of extreme events through statistical bias correction of RCM-driven climate simulations. Hydrology \& Earth System Sciences, 15(8).

Rosenfeld, A. (1998). From Image Analysis to Computer Vision: Motives, Methods, and Milestones.

Rosenfeld, A., \& Weszka, J. S. (1980). Picture recognition. Digital pattern recognition, 135166.

Rutledge, G. K., Alpert, J., \& Ebisuzaki, W. (2006). NOMADS: A climate and weather model archive at the National Oceanic and Atmospheric Administration. Bulletin of the American Meteorological Society, 87(3), 327-342.

Sadegh, M., Moftakhari, H., Gupta, H. V., Ragno, E., Mazdiyasni, O., Sanders, B., Matthew, R., \& AghaKouchak, A. (2018). Multihazard scenarios for analysis of compound extreme events. Geophysical Research Letters, 45(11), 5470-5480.

Sadegh, M., Ragno, E., \& AghaKouchak, A. (2017). Multivariate C opula A nalysis T oolbox (MvCAT): describing dependence and underlying uncertainty using a B ayesian framework. Water Resources Research, 53(6), 5166-5183.

Saghebian, S. M., Sattari, M. T., Mirabbasi, R., \& Pal, M. (2014). Ground water quality classification by decision tree method in Ardebil region, Iran. Arabian journal of geosciences, 7(11), 4767-4777. 
Salio, P., Hobouchian, M. P., Skabar, Y. G., \& Vila, D. (2015). Evaluation of high-resolution satellite precipitation estimates over southern South America using a dense rain gauge network. Atmospheric Research, 163, 146-161.

Salvadori, G., \& De Michele, C. (2004). Frequency analysis via copulas: Theoretical aspects and applications to hydrological events. Water Resources Research, 40(12).

Salvadori, G., Tomasicchio, G., \& D'Alessandro, F. (2014). Practical guidelines for multivariate analysis and design in coastal and off-shore engineering. Coastal engineering, 88, 1-14.

Sangati, M., Borga, M., Rabuffetti, D., \& Bechini, R. (2009). Influence of rainfall and soil properties spatial aggregation on extreme flash flood response modelling: an evaluation based on the Sesia river basin, North Western Italy. Advances in Water Resources, 32(7), 1090-1106.

Sarhadi, A., Ausín, M. C., \& Wiper, M. P. (2016). A new time-varying concept of risk in a changing climate. Scientific Reports, 6, 35755.

Sarhadi, A., Ausín, M. C., Wiper, M. P., Touma, D., \& Diffenbaugh, N. S. (2018). Multidimensional risk in a nonstationary climate: Joint probability of increasingly severe warm and dry conditions. Science advances, 4(11), eaau3487.

Sarhadi, A., \& Soulis, E. D. (2017). Time - varying extreme rainfall intensity - duration frequency curves in a changing climate. Geophysical Research Letters, 44(5), 24542463.

Sato, T., Kimura, F., \& Kitoh, A. (2007). Projection of global warming onto regional precipitation over Mongolia using a regional climate model. Journal of Hydrology, 333(1), 144-154.

Scaife, A. A., Folland, C. K., Alexander, L. V., Moberg, A., \& Knight, J. R. (2008). European climate extremes and the North Atlantic Oscillation. Journal of Climate, 21(1), 72-83.

Schaake, J. C., Hamill, T. M., Buizza, R., \& Clark, M. (2007). HEPEX: the hydrological ensemble prediction experiment. Bulletin of the American Meteorological Society, $88(10), 1541-1548$.

Schaefer, M. G. (1990). Regional analyses of precipitation annual maxima in Washington State. Water Resources Research, 26(1), 119-131.

Schwarz, G. (1978). Estimating the dimension of a model. The annals of statistics, 6(2), 461464.

Scussolini, P., Tran, T. V. T., Koks, E., Diaz - Loaiza, A., Ho, P. L., \& Lasage, R. (2017). Adaptation to sea level rise: a multidisciplinary analysis for Ho Chi Minh City, Vietnam. Water Resources Research, 53(12), 10841-10857.

Séférian, R., Berthet, S., Yool, A., Palmieri, J., Bopp, L., Tagliabue, A., Kwiatkowski, L., Aumont, O., Christian, J., \& Dunne, J. (2020). Tracking improvement in simulated marine biogeochemistry between CMIP5 and CMIP6. Current Climate Change Reports, 1-25.

Segond, M. L., Onof, C., \& Wheater, H. S. (2006). Spatial-temporal disaggregation of daily rainfall from a generalized linear model. Journal of Hydrology, 331(3-4), 674-689. 
Sen, P. K. (1968). Estimates of the regression coefficient based on Kendall's tau. Journal of the American Statistical Association, 63(324), 1379-1389.

Seneviratne, S., Nicholls, N., Easterling, D., Goodess, C., Kanae, S., Kossin, J., Luo, Y., Marengo, J., McInnes, K., \& Rahimi, M. (2012). Changes in climate extremes and their impacts on the natural physical environment.

Shi, J., \& Malik, J. (2000). Normalized cuts and image segmentation. IEEE Transactions on Pattern Analysis and Machine Intelligence, 22(8), 888-905.

Shimizu, M. H., Ambrizzi, T., \& Liebmann, B. (2017). Extreme precipitation events and their relationship with ENSO and MJO phases over northern South America. International Journal of Climatology, 37(6), 2977-2989.

Shirai, Y. (1979). Three-dimensional computer vision. In Computer vision and sensor-based robots (pp. 187-205): Springer.

Shukla, P., Skea, J., Calvo Buendia, E., Masson-Delmotte, V., Pörtner, H., Roberts, D., Zhai, P., Slade, R., Connors, S., \& Van Diemen, R. (2019). IPCC, 2019: Climate Change and Land: an IPCC special report on climate change, desertification, land degradation, sustainable land management, food security, and greenhouse gas fluxes in terrestrial ecosystems.

Shukla, R. K., Trivedi, M., \& Kumar, M. (2012). On the proficient use of GEV distribution: a case study of subtropical monsoon region in India. arXiv preprint arXiv:1203.0642.

Shum, H.-Y., Chan, S.-C., \& Kang, S. B. (2008). Image-based rendering: Springer Science \& Business Media.

Silver, M., Karnieli, A., Marra, F., \& Fredj, E. (2019). An evaluation of weather radar adjustment algorithms using synthetic data. Journal of Hydrology, 576, 408-421.

Sinclair, S., \& Pegram, G. (2005). Combining radar and rain gauge rainfall estimates using conditional merging. Atmospheric Science Letters, 6(1), 19-22.

Skamarock, W. C., Klemp, J. B., \& Dudhia, J. (2001). Prototypes for the WRF (Weather Research and Forecasting) model. Paper presented at the Preprints, Ninth Conf. Mesoscale Processes, J11-J15, Amer. Meteorol. Soc., Fort Lauderdale, FL.

Sklar, M. (1959). Fonctions de repartition an dimensions et leurs marges. Publ. inst. statist. univ. Paris, 8, 229-231.

Smirnov, N. (1948). Table for estimating the goodness of fit of empirical distributions. The annals of mathematical statistics, 19(2), 279-281.

Smith, R. C., \& Cheeseman, P. (1986). On the representation and estimation of spatial uncertainty. The international journal of Robotics Research, 5(4), 56-68.

Sohn, B., Han, H.-J., \& Seo, E.-K. (2010). Validation of satellite-based high-resolution rainfall products over the Korean Peninsula using data from a dense rain gauge network. Journal of Applied Meteorology and Climatology, 49(4), 701-714.

Sokol, Z., Szturc, J., Orellana-Alvear, J., Popová, J., Jurczyk, A., \& Célleri, R. (2021). The role of weather radar in rainfall estimation and its application in meteorological and hydrological modelling-A Review. Remote Sensing, 13(3), 351. 
Son, C., Lee, T., \& Kwon, H. H. (2017). Integrating nonstationary behaviors of typhoon and nontyphoon extreme rainfall events in East Asia. Scientific Reports, 7, 1-9.

Sonali, P., \& Kumar, D. N. (2013). Review of trend detection methods and their application to detect temperature changes in India. Journal of Hydrology, 476, 212-227.

Song, X., Zhang, J., AghaKouchak, A., Roy, S. S., Xuan, Y., Wang, G., He, R., Wang, X., \& Liu, C. (2014). Rapid urbanization and changes in spatiotemporal characteristics of precipitation in Beijing metropolitan area. Journal of Geophysical Research: Atmospheres, 119(19), 11,250-211,271.

Spencer, P., Faulkner, D., Perkins, I., Lindsay, D., Dixon, G., Parkes, M., Lowe, A., Asadullah, A., Hearn, K., \& Gaffney, L. (2018). The floods of December 2015 in northern England: description of the events and possible implications for flood hydrology in the UK. Hydrology Research, 49(2), 568-596.

Stern, R. D., \& Coe, R. (1984). A model fitting analysis of daily rainfall data. Journal of the Royal Statistical Society: Series A (General), 147(1), 1-18.

Stouffer, R. J., Eyring, V., Meehl, G. A., Bony, S., Senior, C., Stevens, B., \& Taylor, K. (2017). CMIP5 scientific gaps and recommendations for CMIP6. Bulletin of the American Meteorological Society, 98(1), 95-105.

Stowell, D., \& Plumbley, M. D. (2014). Automatic large-scale classification of bird sounds is strongly improved by unsupervised feature learning. PeerJ, 2, e488.

Survey, O. (1946). A Brief Description of the National Grid and Reference System. Retrieved from

Svensson, C., \& Rakhecha, P. (1998). Estimation of probable maximum precipitation for dams in the Hongru River catchment, China. Theoretical and Applied Climatology, 59(1-2), 79-91.

Szeliski, R. (1999). A multi-view approach to motion and stereo. Paper presented at the Proceedings. 1999 IEEE Computer Society Conference on Computer Vision and Pattern Recognition (Cat. No PR00149).

Szeliski, R. (2010). Computer vision: algorithms and applications: Springer Science \& Business Media.

Szulczewski, W., \& Jakubowski, W. (2018). The Application of Mixture Distribution for the Estimation of Extreme Floods in Controlled Catchment Basins. Water Resources Management, 32(10), 3519-3534.

Tanguy, M., Dixon, H., Prosdocimi, I., Morris, D. G., \& Keller, V. D. J. (2016). Gridded estimates of daily and monthly areal rainfall for the United Kingdom (18902015)[CEH-GEAR]. NERC Environmental Information Data Centre.

Tehrany, M. S., Pradhan, B., \& Jebur, M. N. (2013). Spatial prediction of flood susceptible areas using rule based decision tree (DT) and a novel ensemble bivariate and multivariate statistical models in GIS. Journal of Hydrology, 504, 69-79.

Teng, J., Jakeman, A. J., Vaze, J., Croke, B. F., Dutta, D., \& Kim, S. (2017). Flood inundation modelling: A review of methods, recent advances and uncertainty analysis. Environmental Modelling \& Software, 90, 201-216. 
Ter Braak, C. J. (2006). A Markov Chain Monte Carlo version of the genetic algorithm Differential Evolution: easy Bayesian computing for real parameter spaces. Statistics and Computing, 16(3), 239-249.

Terzopoulos, D. (1983). Multilevel computational processes for visual surface reconstruction. Computer Vision, Graphics, and Image Processing, 24(1), 52-96.

Thi, H. P., Quoc, D. N., Hoai, T. T. T., \& Le Thi, N. (2019). Determination of the relationship between Vietnam national coordinate reference system (VN-2000) and ITRS, WGS84 and PZ-90. Paper presented at the E3S Web of Conferences.

Thompson, J. (1952). On the operational deficiences in categorical weather forecasts. Bulletin of the American Meteorological Society, 33(6), 223-226.

Thorndahl, S., Einfalt, T., Willems, P., Nielsen, J. E., ten Veldhuis, M.-C., Arnbjerg-Nielsen, K., Rasmussen, M. R., \& Molnar, P. (2017). Weather radar rainfall data in urban hydrology. Hydrology and Earth System Sciences, 21(3), 1359-1380.

Thuc, T., Van Thang, N., Huong, H. T. L., Van Khiem, M., Hien, N. X., \& Phong, D. H. (2016). Climate change and sea level rise scenarios for Vietnam. Ministry of Natural resources and Environment. Hanoi, Vietnam.

Todini, E. (2017). Flood forecasting and decision making in the new millennium. Where are we? Water Resources Management, 31(10), 3111-3129.

Tramblay, Y., Neppel, L., Carreau, J., \& Najib, K. (2013). Non-stationary frequency analysis of heavy rainfall events in southern France. Hydrological Sciences Journal, 58(2), 280294.

Tran, T. V. T., \& Chau, N. X. Q. (2015). Forecasting inundation hazard in Ho Chi Minh City due to the impacts of spring tide and flood from upstream reservoirs. Science and Technology Journal of Agriculture \& Rural Development Vietnam, 5, 3-10.

Trenberth, K. E., \& Stepaniak, D. P. (2001). Indices of el Niño evolution. Journal of Climate, 14(8), 1697-1701.

Tsangaratos, P., \& Ilia, I. (2016). Landslide susceptibility mapping using a modified decision tree classifier in the Xanthi Perfection, Greece. Landslides, 13(2), 305-320.

Tversky, A., \& Kahneman, D. (1992). Advances in prospect theory: Cumulative representation of uncertainty. Journal of Risk and Uncertainty, 5(4), 297-323.

Uppala, S. M., Kållberg, P. W., Simmons, A. J., Andrae, U., Bechtold, V. D. C., Fiorino, M., Gibson, J. K., Haseler, J., Hernandez, A., \& Kelly, G. A. (2005). The ERA - 40 re analysis. Quarterly Journal of the Royal Meteorological Society: A journal of the atmospheric sciences, applied meteorology and physical oceanography, 131(612), 2961-3012.

Urdiales, D., Meza, F., Gironás, J., \& Gilabert, H. (2018). Improving stochastic modelling of daily rainfall using the ENSO index: Model development and application in Chile. Water, 10(2), 145.

US Weather Bureau. (1958). Rainfall intensity-frequency regime. Technical paper, 29. 
Vachaud, G., Quertamp, F., Phan, T. S. H., Ngoc, T. D. T., Nguyen, T., Luu, X. L., Nguyen, A. T., \& Gratiot, N. (2019). Flood-related risks in Ho Chi Minh City and ways of mitigation. Journal of Hydrology, 573, 1021-1027.

van den Hurk, B., van Meijgaard, E., de Valk, P., van Heeringen, K.-J., \& Gooijer, J. (2015). Analysis of a compounding surge and precipitation event in the Netherlands. Environmental Research Letters, 10(3), 035001.

Vélez, C., Alfonso, L., Sánchez, A., Galvis, A., \& Sepúlveda, G. (2014). Centinela: an early warning system for the water quality of the Cauca River. Journal of Hydroinformatics, 16(6), 1409-1424.

Villalobos-Herrera, R., Bevacqua, E., Ribeiro, A. F., Auld, G., Crocetti, L., Mircheva, B., Ha, M., Zscheischler, J., \& De Michele, C. (2021). Towards a compound-event-oriented climate model evaluation: a decomposition of the underlying biases in multivariate fire and heat stress hazards. Natural Hazards and Earth System Sciences, 21(6), 1867-1885.

Viviroli, D., Weingartner, R., \& Messerli, B. (2003). Assessing the hydrological significance of the world's mountains. Mountain Research and Development, 23(1), 32-40.

Von Storch, H. (1999). Misuses of statistical analysis in climate research. In Analysis of climate variability (pp. 11-26): Springer.

Vu, T. T., Nguyen, P. K., Chua, L. H., \& Law, A. W. (2015). Two-dimensional hydrodynamic modelling of flood inundation for a part of the Mekong River with TELEMAC-2D. British Journal of Environment and Climate Change, 5(2), 162-175.

Waliser, D., Gleckler, P. J., Ferraro, R., Taylor, K. E., Ames, S., Biard, J., Bosilovich, M. G., Brown, O., Chepfer, H., \& Cinquini, L. (2020). Observations for model Intercomparison project (Obs4MIPs): Status for CMIP6. Geoscientific Model Development, 13(7), 2945-2958.

Wang, H. (2017). Exploring decision-making process in the context of operational flood forecasting by using Prospect Theory and Decision Trees. (Master of Science). Delft. (17-07)

Wang, P. (1987). Residual plots for detecting nonlinearity in generalized linear models. Technometrics, 29(4), 435-438.

Warmerdam, F. (2008). The geospatial data abstraction library. In Open source approaches in spatial data handling (pp. 87-104): Springer.

Weigel, A. P., Knutti, R., Liniger, M. A., \& Appenzeller, C. (2010). Risks of model weighting in multimodel climate projections. Journal of Climate, 23(15), 4175-4191.

Weigel, A. P., Liniger, M., \& Appenzeller, C. (2008). Can multi - model combination really enhance the prediction skill of probabilistic ensemble forecasts? Quarterly Journal of the Royal Meteorological Society: A journal of the atmospheric sciences, applied meteorology and physical oceanography, 134(630), 241-260.

Wendland, W. M. (1987). Hydroclimatology. In Climatology (pp. 497-502). Boston, MA: Springer US.

Westra, S., Alexander, L. V., \& Zwiers, F. W. (2013). Global increasing trends in annual maximum daily precipitation. Journal of Climate, 26(11), 3904-3918. 
Westra, S., Fowler, H. J., Evans, J. P., Alexander, L. V., Berg, P., Johnson, F., Kendon, E. J., Lenderink, G., \& Roberts, N. M. (2014). Future changes to the intensity and frequency of short - duration extreme rainfall. Reviews of Geophysics, 52(3), 522-555.

Weszka, J. S., \& Rosenfeld, A. (1978). Threshold evaluation techniques. IEEE Transactions on Systems, Man, and Cybernetics, 8(8), 622-629.

Wolberg, G. (1998). Image morphing: a survey. The visual computer, 14(8), 360-372.

Won, J., Choi, J., Lee, O., \& Kim, S. (2020). Copula-based Joint Drought Index using SPI and EDDI and its application to climate change. Science of the Total Environment, 744, 140701.

Wu, S., Angelikopoulos, P., Papadimitriou, C., \& Koumoutsakos, P. (2018). Bayesian annealed sequential importance sampling: an unbiased version of transitional Markov chain Monte Carlo. ASCE-ASME Journal of Risk and Uncertainty in Engineering Systems, Part B: Mechanical Engineering, 4(1).

Wu, W., Tang, X.-P., Guo, N.-J., Yang, C., Liu, H.-B., \& Shang, Y.-F. (2013). Spatiotemporal modeling of monthly soil temperature using artificial neural networks. Theoretical and Applied Climatology, 113(3), 481-494.

Xin, X., Wu, T., Zhang, J., Yao, J., \& Fang, Y. (2020). Comparison of CMIP6 and CMIP5 simulations of precipitation in China and the East Asian summer monsoon. International Journal of Climatology, 40(15), 6423-6440.

Xu, C., He, H. S., Hu, Y., Chang, Y., Li, X., \& Bu, R. (2005). Latin hypercube sampling and geostatistical modeling of spatial uncertainty in a spatially explicit forest landscape model simulation. Ecological Modelling, 185(2-4), 255-269.

Xu, Z., Han, Y., \& Yang, Z. (2019). Dynamical downscaling of regional climate: A review of methods and limitations. Science China Earth Sciences, 62(2), 365-375.

Yadav, R., Ramu, D., \& Dimri, A. (2013). On the relationship between ENSO patterns and winter precipitation over North and Central India. Global and Planetary Change, 107, 50-58.

Yan, Z., Bate, S., Chandler, R. E., Isham, V., \& Wheater, H. (2002). An analysis of daily maximum wind speed in northwestern Europe using generalized linear models. Journal of Climate, 15(15), 2073-2088.

Yang, C., Chandler, R. E., Isham, V. S., \& Wheater, H. S. (2005). Spatial - temporal rainfall simulation using generalized linear models. Water Resources Research, 41(11).

Yoon, P., Kim, T.-W., \& Yoo, C. (2013). Rainfall frequency analysis using a mixed GEV distribution: a case study for annual maximum rainfalls in South Korea. Stochastic Environmental Research and Risk Assessment, 27(5), 1143-1153.

Yoon, S., Kumphon, B., \& Park, J.-S. (2015). Spatial Modelling of Extreme Rainfall in Northeast Thailand. Procedia Environmental Sciences, 26, 45-48.

Yu, J. Y., \& Kim, S. T. (2010). Identification of central - Pacific and eastern - Pacific types of ENSO in CMIP3 models. Geophysical Research Letters, 37(15). 
Yue, S., Pilon, P., \& Cavadias, G. (2002). Power of the Mann-Kendall and Spearman's rho tests for detecting monotonic trends in hydrological series. Journal of Hydrology, 259(1-4), 254-271.

Yunus, R. M., Hasan, M. M., Razak, N. A., Zubairi, Y. Z., \& Dunn, P. K. (2017). Modelling daily rainfall with climatological predictors: Poisson - gamma generalized linear modelling approach. International Journal of Climatology, 37(3), 1391-1399.

Zar, J. H. (2005). Spearman rank correlation. Encyclopedia of biostatistics, 7.

Zhan, L.-J., \& Qin, C.-Z. (2012). Parallel geospatial raster processing by Geospatial Data Abstraction Library (GDAL) - applicability and defects. Transactions in GIS.

Zhang, L., \& Singh, V. (2006). Bivariate flood frequency analysis using the copula method. Journal of hydrologic engineering, 11(2), 150-164.

Zhao, T., Li, C., \& Zuo, Z. (2016). Contributions of anthropogenic and external natural forcings to climate changes over China based on CMIP5 model simulations. Science China Earth Sciences, 59(3), 503-517.

Zhongming, Z., Linong, L., Wangqiang, Z., \& Wei, L. (2021). Water-related hazards dominate disasters in the past 50 years.

Zhu, Y., Liu, Y., Wang, W., Singh, V. P., Ma, X., \& Yu, Z. (2019). Three dimensional characterization of meteorological and hydrological droughts and their probabilistic links. Journal of Hydrology, 578, 124016.

Zscheischler, J., Fischer, E. M., \& Lange, S. (2019). The effect of univariate bias adjustment on multivariate hazard estimates. Earth System Dynamics, 10(1), 31-43.

Zscheischler, J., Westra, S., Van Den Hurk, B. J., Seneviratne, S. I., Ward, P. J., Pitman, A., AghaKouchak, A., Bresch, D. N., Leonard, M., \& Wahl, T. (2018). Future climate risk from compound events. Nature Climate Change, 8(6), 469-477.

Zucker, S. W. (1976). Toward a model of texture. Computer Graphics and Image Processing, 5(2), 190-202. 


\section{Appendix Data Processing and Toolbox Access}

\section{A.1 Data extraction and HPC access}

To extract each grid data in the ROI defined by the SRS-GDA toolbox, high-performance computing resources provided by HPC-Wales is employed for processing such huge data parallelly. The program running at the assigned computation nodes is written in Python.

For extraction from the GEAR dataset:

from multiprocessing import Process

from netCDF4 import Dataset

import scipy.io as sio

import os

$\mathrm{m}=113$ \#year

Ex $=$ 'Rainfall $\_$

domain = os.path.abspath('/scratch/s.966992/Rainfall_UK')

domain1 = os.path.abspath('/lustrehome/home/s.966992/Catchment')

info1 = os.listdir('/lustrehome/home/s.966992/Catchment')

$\mathrm{N}=88 \#=88$ for location group; $=74$ for shape group $;=81$ for size group .

def extract_p(mat_fid,matName):

P_Data $1=[\text { None }]^{*} \operatorname{len}\left(\right.$ mat_fid $\left.\left[{ }^{\prime} X Y^{\prime}\right][:]\right)$

for $\mathrm{k}$ in $\operatorname{range}\left(\mathrm{len}\left(\mathrm{P}_{-}\right.\right.$Data 1$\left.)\right)$ : 
P_Data1 $[\mathrm{k}]=[0.0]^{*} \mathrm{~m}$

for $\mathrm{i}$ in range(0,len(mat_fid['XY'][:])):

$\mathrm{XY}=$ mat_fid['XY'][i]

count $=0$

for info in os.listdir('/scratch/s.966992/Rainfall_UK'): \# extract rainfall(nc file)

info $=$ os.path.join $($ domain,info $)$

nc_fid $=$ Dataset $($ info,,$r$ ')

P_Data1 $[i][$ count $]=$ nc_fid.variables['rainfall_amount'][:365,XY[1],XY[0]]

count $+=1$

nc_fid.close()

adict $=\{\}$

$\operatorname{adict}[$ 'P_Data'] $=$ P_Data1

sio.savemat $(\mathrm{Ex}+$ matName, adict $)$

def proccounter(counter):

infol 1 = os.path.join (domain1,info1[counter] $)$

matName $=$ os.path.basename $($ info 11$)$

mat_fid $=$ sio.loadmat $($ infol1 $)$

extract_p(mat_fid,matName)

return counter

if __name__ = ='_main_':

for counter in range $(\mathrm{N})$ :

Process $($ target $=$ proccounter, $\operatorname{args}=($ counter,$)) \cdot \operatorname{start}()$

$\#$ pool $=$ Pool () 
\#for counter in pool.map(proccounter, range $(\mathrm{N}))$ :

\# pool.start()

\#pool.join()

For extraction from ADAM dataset:

$\mathrm{m}=129$ \#year

$\mathrm{Ex}=$ 'Rainfall ${ }_{-}^{\prime}$

domain = os.path.abspath('/lustrehome/home/s.966992/Rainfall_AU')

domain1 = os.path.abspath('/lustrehome/home/s.966992/Size')

info1 $=$ os.listdir('/lustrehome/home/s.966992/Size')

P_Data $=[\text { None }]^{*} \mathrm{n} \# \mathrm{n}=1$ for location group; $=7$ for shape group; $=10$ for size group.

$\mathrm{N}=691 \#=691$ for location group; $=378$ for shape group; $=627$ for size group.

def extract_p(mat_fid,matName):

for $\mathrm{j}$ in range $(0,10)$ :

P_Data1 $=[\text { None }]^{*}$ len $\left(\right.$ mat_fid $\left.\left[{ }^{\prime} X Y \_S i z e '\right][0][j][:]\right)$

for $\mathrm{k}$ in range(len(P_Data1)):

P_Data1 $[\mathrm{k}]=[0.0]^{*} \mathrm{~m}$

for $\mathrm{i}$ in range $(0$, len(mat_fid['XY_Size'][0][j][:])):

$X Y=$ mat_fid['XY_Size'][0][j][i]

count $=0$;

for info in os.listdir('/lustrehome/home/s.966992/Rainfall_AU'): \# extract rainfall(nc

file)

info $=$ os.path.join (domain,info $)$

nc $\_$fid $=$Dataset $\left(\right.$info, ${ }^{\prime}$ ' $)$

P_Data1 $[i][$ count $]=$ nc_fid.variables['daily_rain'][:365,682-XY[1],XY[0]]

count $+=1$ 


$$
\begin{gathered}
\text { nc_fid.close }() \\
\text { P_Data[j] }=\text { P_Data1 } \\
\operatorname{adict~}=\{\} \\
\text { adict['P_Data'] = P_Data } \\
\text { sio.savemat(Ex+matName, adict })
\end{gathered}
$$

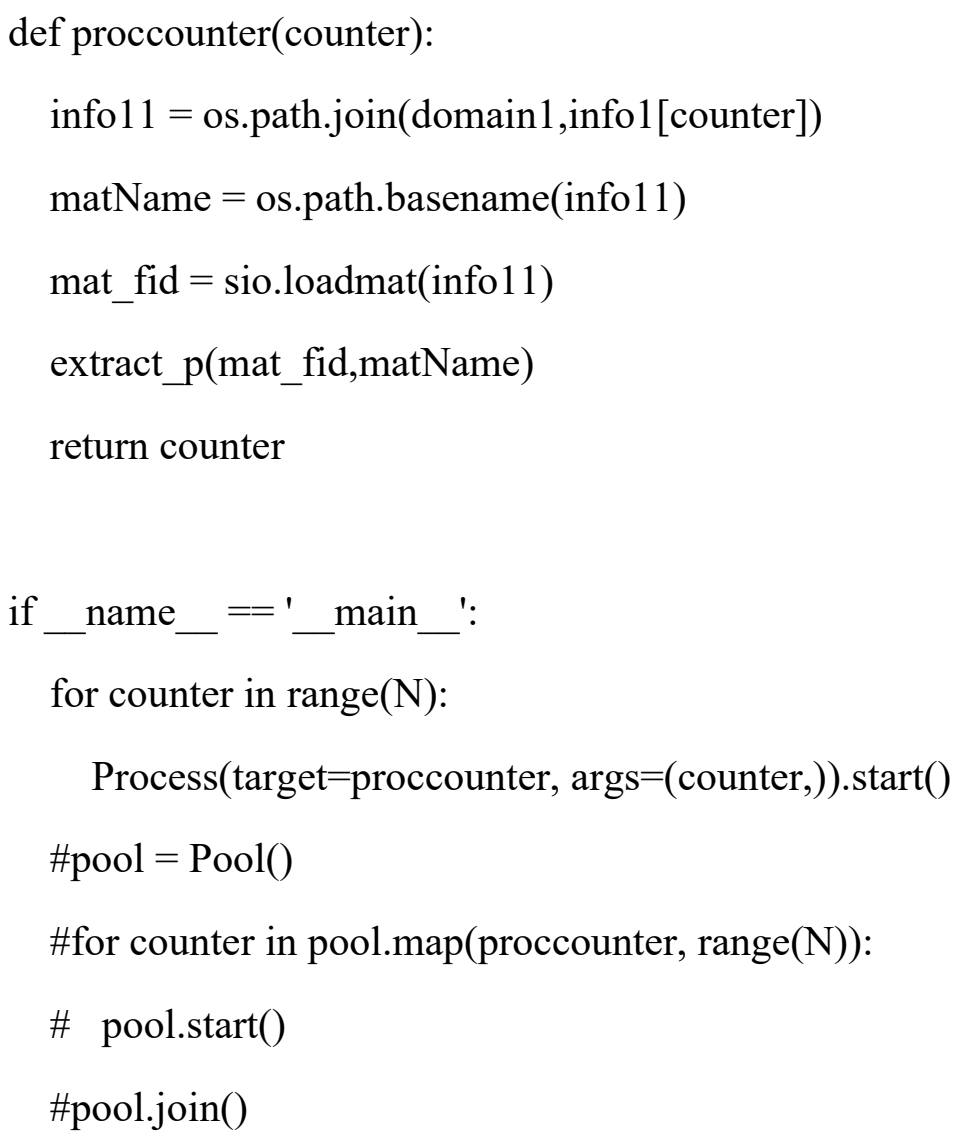

\section{A.2 Toolbox access}

There are two toolboxes developed in the thesis: 1) the open-source toolbox of spatial random sampling for grid-based data analysis (SRS-GDA toolbox, doi: 10.5281/zenodo.4044626) whose source code as well as the example case are available at the GitHub (https://github.com/wanghan924/SRS-GDA Toolbox.git); 2) the open-source toolbox of 
spatial pattern extraction and recognition (SPER) whose source code and GUI software are available at the GitHub (https://github.com/wanghan924/SPER-toolbox). Both source codes are provided subject to a GPL V3 licence. Use/fork of the toolbox is subject to proper acknowledgement as stated on the Webpage of the toolbox.

\section{A.3 Model selection and parameter estimation}

Table A1 presents the details of parameter values of two marginal distributions (monthly maximum skew surge, MMS and monthly maximum rainfall, MMR) estimated by both stationary and nonstationary models and the best model is selected by comparing the criteria (AIC and BIC). It should be noted that only time series with a significant trend evaluated by Bootstrapping Mann-Kendall test is further checked with the nonstationary models. And Table A2 presents the selection of copula from the candidates according to the AIC.

Table A1 The parameters of the marginal distribution of MMS and MMR under both stationary and nonstationary conditions.

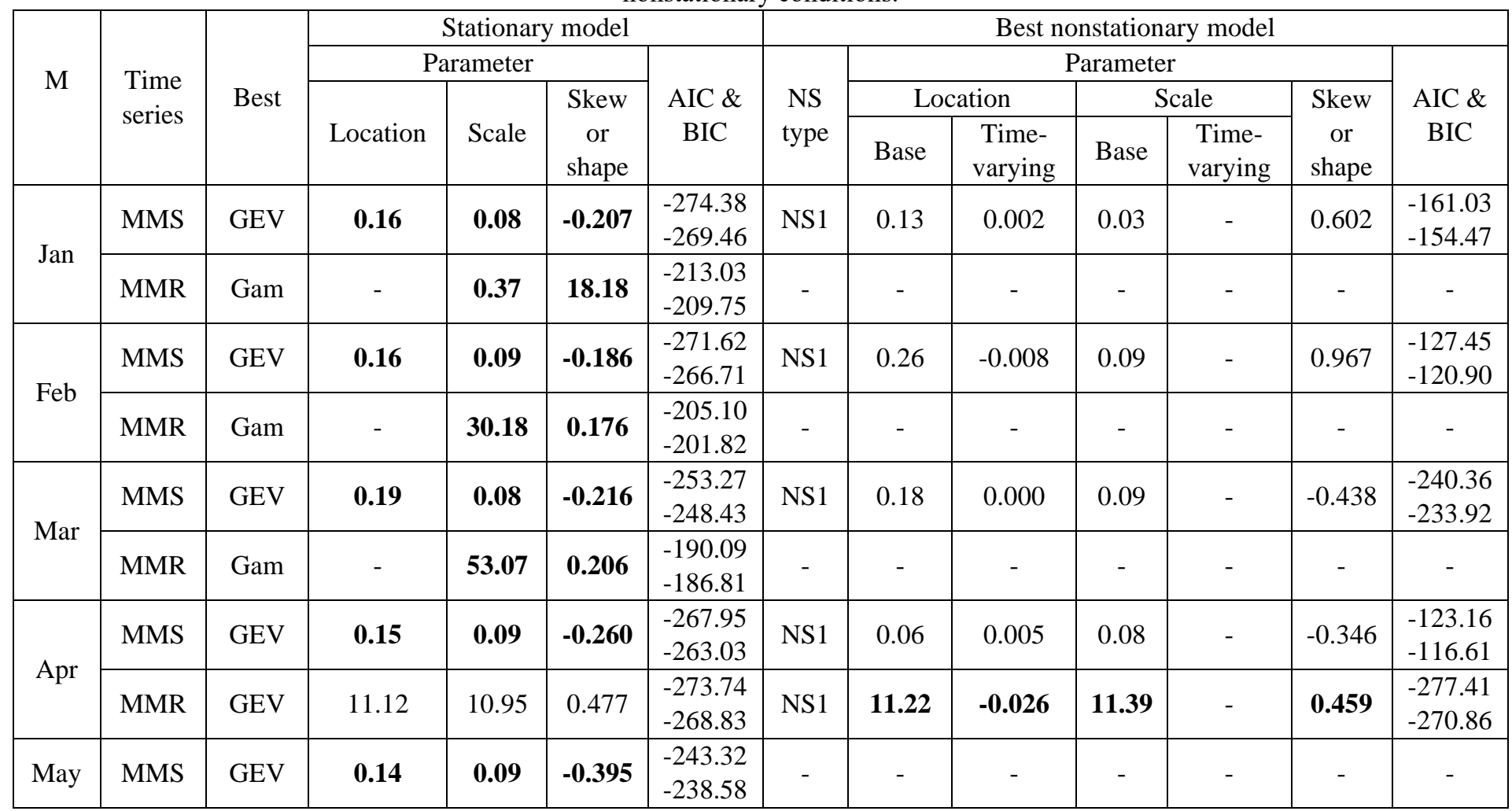




\begin{tabular}{|c|c|c|c|c|c|c|c|c|c|c|c|c|c|}
\hline & MMR & GEV & 32.14 & 15.26 & 0.016 & $\begin{array}{l}-210.94 \\
-206.02\end{array}$ & - & - & - & - & - & - & - \\
\hline \multirow{2}{*}{ Jun } & MMS & $\log N$ & -2.03 & 0.56 & - & $\begin{array}{l}-227.39 \\
-230.56\end{array}$ & NS1 & -2.06 & 0.002 & 0.44 & - & - & $\begin{array}{l}-218.28 \\
-213.53\end{array}$ \\
\hline & MMR & GEV & 34.71 & 13.37 & 0.131 & $\begin{array}{l}-249.82 \\
-244.91\end{array}$ & - & - & - & - & - & - & - \\
\hline \multirow{2}{*}{ Jul } & MMS & GEV & 0.16 & 0.08 & -0.253 & $\begin{array}{l}-262.94 \\
-258.03\end{array}$ & NS1 & 0.16 & 0.000 & 0.06 & - & -0.044 & $\begin{array}{l}-224.73 \\
-218.18\end{array}$ \\
\hline & MMR & $\log N$ & 3.70 & 0.31 & - & $\begin{array}{l}-243.07 \\
-239.79\end{array}$ & - & - & - & - & - & - & - \\
\hline \multirow{2}{*}{ Aug } & MMS & GEV & 0.17 & 0.08 & -0.534 & $\begin{array}{l}-215.36 \\
-210.44\end{array}$ & NS1 & 0.11 & 0.001 & 0.12 & - & -0.697 & $\begin{array}{l}-154.18 \\
-147.63\end{array}$ \\
\hline & MMR & $\log N$ & 3.84 & $\mathbf{0 . 3 9}$ & - & $\begin{array}{r}-262.00 \\
-258.72\end{array}$ & - & - & - & - & - & - & - \\
\hline \multirow{2}{*}{ Sep } & MMS & GEV & 0.154 & 0.080 & -0.309 & $\begin{array}{l}-245.23 \\
-240.31\end{array}$ & NS1 & 0.09 & 0.003 & 0.10 & - & -0.442 & $\begin{array}{l}-148.46 \\
-141.91\end{array}$ \\
\hline & MMR & GEV & 40.62 & 17.74 & 0.161 & $\begin{array}{l}-254.44 \\
-249.53 \\
\end{array}$ & - & - & - & - & - & - & - \\
\hline \multirow{2}{*}{ Oct } & MMS & GEV & 0.18 & 0.09 & -0.405 & $\begin{array}{l}-231.68 \\
-226.76\end{array}$ & NS1 & 0.28 & -0.008 & 0.15 & - & 0.510 & $\begin{array}{l}-134.52 \\
-127.97\end{array}$ \\
\hline & MMR & GEV & 42.29 & 14.44 & 0.010 & $\begin{array}{l}-253.10 \\
-248.18\end{array}$ & NS1 & 42.82 & -0.084 & 16.40 & - & 0.045 & $\begin{array}{l}-264.93 \\
-258.38\end{array}$ \\
\hline \multirow{2}{*}{ Nov } & MMS & GEV & 0.16 & 0.08 & -0.341 & $\begin{array}{l}-229.52 \\
-224.69\end{array}$ & NS1 & 0.15 & 0.000 & 0.09 & - & -0.785 & $\begin{array}{l}-216.51 \\
-210.06\end{array}$ \\
\hline & MMR & GEV & 25.63 & 14.17 & 0.061 & $\begin{array}{l}-238.17 \\
-229.98\end{array}$ & NS3 & 27.64 & $-\mathbf{0 . 1 3 9}$ & 2.47 & 0.012 & 0.012 & $\begin{array}{l}-263.65 \\
-258.73\end{array}$ \\
\hline \multirow{2}{*}{ Dec } & MMS & GEV & 0.14 & 0.09 & -0.453 & $\begin{array}{r}-237.48 \\
-232.57\end{array}$ & NS1 & 0.05 & 0.004 & 0.07 & - & -0.196 & $\begin{array}{l}-127.81 \\
-121.26 \\
\end{array}$ \\
\hline & MMR & GEV & 9.21 & 7.79 & 0.132 & $\begin{array}{l}-243.04 \\
-238.13\end{array}$ & - & - & - & - & - & - & - \\
\hline
\end{tabular}

Please note that "Gam" is short for Gamma distribution, "LogN" is short for Lognormal distribution, and "GEV" is short for Generalised extreme value distribution.

Table A2 The comparison among copula candidates.

\begin{tabular}{|c|c|c|c|c|c|c|c|c|c|}
\hline \multirow{2}{*}{$\begin{array}{l}\text { Time } \\
\text { series }\end{array}$} & \multicolumn{8}{|c|}{ AIC } & \multirow{2}{*}{ Selection } \\
\hline & Gaussian & Clayton & Frank & Gumbel & Indepen & Joe & Plackett & Raftery & \\
\hline Jan & -242.0 & -241.7 & -242.2 & -241.7 & -243.7 & -241.7 & -242.2 & -241.7 & Indepen \\
\hline Feb & -177.3 & -177.5 & -177.3 & -177.1 & -177.7 & -176.8 & -177.2 & -177.5 & Indepen \\
\hline Mar & -178.6 & -178.4 & -177.8 & -177.0 & -178.4 & -176.6 & -177.7 & -178.4 & Gaussian \\
\hline Apr & -269.1 & -290.8 & -267.5 & -257.9 & -232.9 & $\begin{array}{l}-248.8 \\
\end{array}$ & -267.3 & -289.5 & Clayton \\
\hline May & -234.6 & -234.9 & -234.5 & -234.4 & $\begin{array}{l}-236.4 \\
\end{array}$ & -234.4 & -234.5 & -234.8 & Indepen \\
\hline Jun & -231.9 & -223.1 & -232.0 & -223.1 & -225.2 & -223.1 & -232.0 & -223.1 & Plackett \\
\hline Jul & -252.9 & -246.3 & -253.6 & -246.3 & -253.7 & -246.3 & -248.3 & -246.3 & Indepen \\
\hline Aug & -230.9 & -236.4 & -229.9 & -227.6 & -227.3 & -226.0 & -229.7 & -237.1 & Raftery \\
\hline Sep & -243.321 & -244.7 & -242.8 & -243.2 & -242.6 & -242.9 & -242.8 & -245.1 & Raftery \\
\hline Oct & -247.6 & -239.9 & -246.5 & -251.1 & -229.3 & -254.3 & -246.5 & -239.2 & Joe \\
\hline Nov & -241.3 & -237.8 & -238.7 & -242.7 & -239.2 & -244.4 & -241.5 & -238.9 & Joe \\
\hline
\end{tabular}




\begin{tabular}{|l|l|l|l|l|l|l|l|l|l|}
\hline Dec & -244.4 & -249.4 & -241.8 & -241.5 & -228.1 & -238.1 & -242.1 & $\mathbf{- 2 5 0 . 2}$ & Raftery \\
\hline
\end{tabular}

Please note that "Indepen" is short for "Independence". 